\title{
Structural Studies on Transmembrane
}

\section{Signalling Mechanism of Histidine Kinase}

\author{
CitA
}

\author{
Dissertation \\ for the award of the degree \\ Doctor of Philosophy
}

Division of Mathematics and Natural Sciences

of the Georg-August University Göttingen

within the doctoral program Biomolecules: Structure - Function - Dynamics of the Georg-August University School of Science (GAUSS)

\author{
submitted by \\ Michele Salvi \\ From Casoli, Italy
}

Göttingen 2018 


\section{Thesis Committee}

Prof. Dr. Christian Griesinger

Department of NMR-based Structural Biology, Max Planck Institute for Biophysical Chemistry, Goettingen, Germany

Prof. Dr. Blanche Schwappach

Department of Biochemistry I, University of Göttingen Medical School, Goettingen, Germany

Prof. Dr. Bert de Groot

Research group for Computational Biomolecular Dynamics, Max Planck Institute for Biophysical Chemistry, Goettingen, Germany

\section{Members of the Examination Board}

Reviewer:

Prof. Dr. Christian Griesinger

Department of NMR-based Structural Biology, Max Planck Institute for Biophysical Chemistry, Goettingen, Germany

Second Reviewer: $\quad$ Prof. Dr. Blanche Schwappach

Department of Biochemistry I, University of Göttingen Medical School, Goettingen, Germany

\section{Further members of the Examination Board:}

Prof. Dr. Bert de Groot

Research group for Computational Biomolecular Dynamics, Max Planck Institute for Biophysical Chemistry, Goettingen, Germany

Prof. Dr. Markus Zweckstetter

Research group for Translational Structural Biology in Dementia, German Center for Neurodegenerative Diseases, Goettingen, Germany

Prof. Dr. Claudia Steinem

Institute for Organic and Biomolecular Chemistry, University of Göttingen, Goettingen, Germany

Prof. Dr. Michael Meinecke

Department of Cellular Biochemistry, University of Göttingen Medical School, Goettingen, Germany

Date of the oral examination: January 14th 2019 


\section{Affidavit}

Herewith I declare, that I prepared this thesis titled Structural Studies on Transmembrane Signalling Mechanism of Histidine Kinase CitA on my own and with no other sources and aids than quoted.

Place, Date

Signature 



\section{Related publications}

The work presented in this thesis led to the following publications:

Salvi, M., Schomburg, B., Giller, K., Graf, S., Unden, G., Becker, S., Lange, A. and Griesinger, C. Sensory domain contraction in histidine kinase CitA triggers transmembrane signaling in the membrane-bound sensor. Proceedings of the National Academy of Sciences, p. 3115-3120, 2017. 



\section{Abstract}

Perception of environmental changes and subsequent signal transduction are key aspects for the survival of an organism. In bacteria, two component systems (TCSs) consisting of a homodimeric receptor histidine kinase (HK) and a response regulator $(\mathrm{RR})$ are the prevalent mechanism of stimulus sensing and cell signalling. They regulate motility, metabolism and development, and in addition, they are essential for virulence in a number of pathogenic species. The investigation of the signal transduction in TCSs is therefore essential to deepen our understanding of cell signalling and to develop a new class of antimicrobial drugs.

The Geobacillus thermodenitrificans citrate receptor A (CitA) is used in this study as a model system for HKs. CitA is responsible for the activation of the citrate metabolism together with its cognate regulate receptor CitB. The periplasmic PAS (Per-Arnt-Sim) receptor is able to recognise the citrate molecule. The signal is then transferred across the membrane to a second, cytosolic PAS domain before reaching the conserved kinase core and triggering the auto-phosphorylation reaction. Although both citrate-binding and phosphate transfer has been described thoroughly, the signal relay from one domain to another remain poorly understood. Therefore, a combination of liquid- and solid-state NMR is adopted in this PhD project together with other biophysical techniques, such as X-ray crystallography and fluorescence microscopy, to unveil details of the signalling mechanism of CitA. Our results show that an helical element is formed at the periplasm-membrane interface upon the citrate binding event both in the isolated periplasmic PAS domain and in a liposome-embedded CitA construct. The formation of the C-terminal $\alpha$ helix extends the results previously published on CitA and confirm the piston model proposed for the transmembrane helix motion. In addition, the existence of different dimer arrangements of the cytosolic PAS domain previously observed only in crystal structures is confirmed also in solution. These alternative dimeric conformations are then associated for the first time with the relaying mechanism of the cytosolic PAS, shedding light on the role of the additional signalling domains interposed between the membrane and the kinase core. 



\section{Contents}

Abstract $\quad$ vi

1 Introduction $\quad 1$

1.1 Cellular adaptation to environmental changes . . . . . . . . . . . 1

1.2 Two component systems . . . . . . . . . . . . . . . . . . . . . . . .

1.3 Histidine kinases . . . . . . . . . . . . . . . . . 3

1.3.1 General architecture . . . . . . . . . . . . . 3

1.3.2 Signalling mechanism . . . . . . . . . . . . . 6

1.4 CitA family . . . . . . . . . . . . . . . . . . 8

1.5 PAS domains . . . . . . . . . . . . . . . . . . 10

1.6 NMR approach . . . . . . . . . . . . . . . . . . . . . . . . . . . . . . . . . . 11

1.6.1 Other biophysical techniques . . . . . . . . . . . . 15

2 Material and Methods $\quad 16$

2.1 Liquid- state NMR experiments . . . . . . . . . . . . . . . . . . . . 16

2.1.1 Sample preparation . . . . . . . . . . . . . . 16

2.1.2 General procedures . . . . . . . . . . . . . . . 16

2.1.3 ${ }^{15} \mathrm{~N}$ CEST experiments . . . . . . . . . . . . . . . . . . . . . . . . . . 17

2.1.4 Amide exchange patterns . . . . . . . . . . . . . . . 17

2.2 Secondary chemical shift analysis . . . . . . . . . . . . . . . 18

2.3 Solid-state NMR experiments . . . . . . . . . . . . . . . . . . . . 19

2.3.1 Sample preparation . . . . . . . . . . . . . . . . . . 19

2.3.2 General procedures . . . . . . . . . . . . . . . . . . 19

$2.3 .3{ }^{13} \mathrm{C}$ detected spectra . . . . . . . . . . . . . . 20

2.3.4 ${ }^{1} \mathrm{H}$ detected spectra . . . . . . . . . . . . . . . . 20

2.4 Solvent PRE . . . . . . . . . . . . . . . . . . . . . . 21

2.4.1 sPRE in liquid-state NMR . . . . . . . . . . . . . 24

2.4 .2 sPRE in solid-state NMR . . . . . . . . . . . . . 24

2.5 Structure determination . . . . . . . . . . . . . 25

3 Results $\quad \mathbf{2 6}$

3.1 The periplasmic PAS domain . . . . . . . . . . . . . . 26

3.2 The cytosolic PAS domain . . . . . . . . . . . . . . . . . 27

3.3 The liposome embedded construct CitApc . . . . . . . . . . . . . . . 36

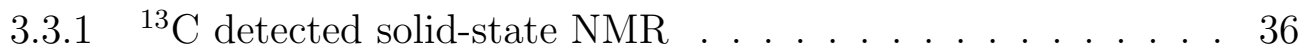

3.3.2 ${ }^{1} \mathrm{H}$ detected approach development . . . . . . . . . . . . 38

3.3.3 ${ }^{1} \mathrm{H}$ detected solid state NMR . . . . . . . . . . . . . . 40

4 Discussion $\quad 48$

4.1 The periplasmic PAS domain . . . . . . . . . . . . . . . 48

4.2 The cytoplasmic PAS domain . . . . . . . . . . . . . . . 52 
4.3 Signalling relay in the $G$. thermodenitrificans CitA . . . . . . . . . 58

4.3.1 Citrate binding and activation of the PASp in context of the membrane . . . . . . . . . . . . . 5 58

4.3.2 The transmembrane region . . . . . . . . . . . . . 59

4.3.3 The role of the cytosolic PAS domain in the CitApc construct 61

Overall visibility . . . . . . . . . . . . . . . . 62

Chemical shift comparison . . . . . . . . . . . . 62

Amide exchange properties . . . . . . . . . . . . 63

Solvent PRE . . . . . . . . . . . . . . . . 66

4.3.4 Compatibility of the antiparallel-to-parallel switch . . . . . . 69

4.4 Conclusion and perspective . . . . . . . . . . . . . 72

$\begin{array}{ll}\text { A Appendix } & \mathbf{7 5}\end{array}$

A. $1{ }^{13} \mathrm{C}-{ }^{15} \mathrm{~N}$ double edited NOESY . . . . . . . . . . . . . . . . 75

A.2 NMR assignments . . . . . . . . . . . . . . . . . . 75

A.3 Pulse programs . . . . . . . . . . . . . . . . . . . . . . . . . . . . . . . . 99

A.3.1 Liquid-state NMR . . . . . . . . . . . . . . . . . . . . . . . 99

A.3.2 ${ }^{13} \mathrm{C}$ detected $\mathrm{ssNMR} \ldots \ldots \ldots \ldots . \ldots . \ldots . . \ldots 105$

A.3.3 ${ }^{1} \mathrm{H}$ detected ssNMR . . . . . . . . . . . . . . . . 105

Bibliography 



\section{Introduction}

\subsection{Cellular adaptation to environmental changes}

The ability to adapt to different environments is crucial to the survival of every organism. As a first step of the adaptation process, the changes (e.g. chemical signals, modifications of osmolarity or of temperature, availability of different nutrients) have to be communicated across the plasma membrane of the cell, where the incoming signals will be processed into a regulatory readout. For this purpose, a variety of receptors are used, including G-protein coupled receptors, membrane channels and catalytic receptors. Amongst the latter are classified the receptor kinases, which respond to an external stimulus with a phosphorylation reaction. Receptor kinases are employed by nearly all kind of organism and feature a kinase domain responsible for the phosphorylation of a specific amino acid (primarily tyrosine, serine/threonine or histidine) found on the receptor itself or on a second protein (Hanson and Schulman, 1992). Phosphorylation is particularly convenient not only because the effects on structural and functional properties are generated by a small protein modification (Westheimer, 1987). First, it is efficient as it makes use of high energy phosphate donor molecules, such as ATP, to guarantee a thermodynamically favourable reaction. Second, it is reversible because of phosphatases which can dephosphorylate the phosphoprotein. As kinases and phosphatases can be triggered in different ways, various regulatory patterns can crosstalk between each other and the activity of all kinases and phosphatases will determine the net state of phosphorylation, which is thus more complex than a 2 state on/off switch (Bray, 1995). Finally, receptor domains that selectively respond to input stimuli are employed to adapt the phosphorylation processing mechanism to various stimuli.

Histidine phosphorylation is the most used phosphorylation mechanism in prokaryotes. It was first reported in mammalian cells over 50 years ago (Boyer et al., 1962), but wide-scale characterization of this protein modification is still lacking due to the instability of phosphorylated histidine at acidic conditions and elevated temperatures (Hultquist, 1968; Hultquist et al., 1966) used in most biochemical and proteomic analyses. In prokaryotes, the first evidence of histidine phosphorylation 
can be credited to Ninfa and Magasanik (1986) and Hess et al. (1987), who studied in vivo the histidine kinases (HKs) Ntrb and CheA, respectively. Since then, the HKs have emerged together with their signalling targets, the response regulators (RRs), as the most abundant signalling system in prokaryotes and are responsible for responding to nutrients, chemoattractants, cell density, temperature changes and more. A few signalling systems based on HKs are also found in eukaryotes, but only in plants, fungi and amoebae (Thomason and Kay, 2000). Since they are apparently absent from the animal kingdom, HKs represent an interesting target for the development of a new class of antimicrobial drugs, which are not directly toxic for the pathogen nor to the host. This new class of drugs would interfere with the HKs-based signalling system leading to the destruction of the bacteria by the host immune system and is less likely to evoke drug resistance compared to classic antibiotics (Matsushita, 2002).

\section{$1.2 \quad$ Two component systems}

Histidine kinases form together with the RRs the so-called two components system (TCS), which are one of the most used signalling systems in prokaryotes. Historically, TCS represent the best studied mode of prokaryotic signal transduction (Kofoid and Parkinson, 1988). A given bacterium can have from ten to hundreds of TCS (Ulrich and Zhulin, 2018) capable to process a wide variety of inputs, such as nutrients, chemoattractants, cell density, temperature changes and many more. The great diversity of inputs is due to the modularity of both HKs and RRs. The HKs are usually membrane-bound proteins with dimeric functional state. They comprise two basic signalling components, namely a sensing domain on the extracytoplasmic (periplasmic or external) and an highly conserved cytoplasmic kinase core. Additional signalling domains can be found in the cytoplasm, between the kinase core and the membrane, or in the periplasm, between the sensing domain and the membrane. Exceptions to this general architecture are HKs which are soluble, such as the chemotaxis kinase CheA (Stock et al., 1988) and the nitrogen regulatory kinase NtrB (Macfarlane and Merrick, 1985). These HKs are not acting as periplasmic membrane receptor, but can be regulated by intracellular stimuli or interactions with cytoplasmic domains of other proteins. Details of architecture and mechanisms of HKs will be introduced later on (see section 1.3.1 and 1.3.2). A soluble $R R$ is crucial to the transduction of the signals to the cytoplasmic 
cell components as most of the HKs are membrane-bound (Cock and Whitworth, 2007). Its general architecture comprises two domains: a conserved N-terminal receiver (REC) domain and a variable C-terminal effector domain. The REC domain has enzymatic activity and catalyses both phosphoryl transfer and autodephosphorylation. It receives the phosphoryl group from the conjugate HK on a highly conserved aspartate. The presence of the phosphoryl group alters the equilibrium between the active and inactive conformation, stabilizing the former and triggering the effector domain. The majority of these effector domains (63\%) bind DNA. Other roles of the effector domains include RNA binding, protein interaction and enzymatic activity (Gao and Stock, 2009).

In addition to the canonical function, RRs that exist as stand-alone REC domains have been isolated. These are either involved in chemotaxis systems by binding directly to motor proteins, or as intermediates in phosphorelay pathways. In the latter, several His-Asp transfer steps are involved, with the first one between the conserved histidine of the HK and the conserved aspartate of the REC domain. In the so-called hybrid HKs, the REC domain can be found as an additional module downstream of the kinase core, as in the HK ArcB (Kazuya et al., 1994). Afterwards, the phosphoryl group is transferred to a histidine phosphotransfer (HPt) domain, which is structurally related to the HK and can be either part of a different protein or of the kinase itself. From here the signal cascade continues and the phosphoryl group can be transferred to another REC domain, for example on a RR or on another intermediate (Ogino et al., 1998; Perego, 1998).

\subsection{Histidine kinases}

\subsubsection{General architecture}

Like most signalling proteins, HKs have a modular architecture with a variety of receptor domains linked to a conserved kinase core. In this way, it is possible to couple many different input signals to appropriate output responses exploiting a conserved phosphotransfer pathway.

In prototypical HKs, the kinase core consists of two distinct domains: the Cterminal catalytic and ATP binding (CA) domain and the dimerization and histidine phosphotransfer (DHp) domain. In the last years, various structures have been solved for the complete kinase core, isolated DHp and isolated CA domains 


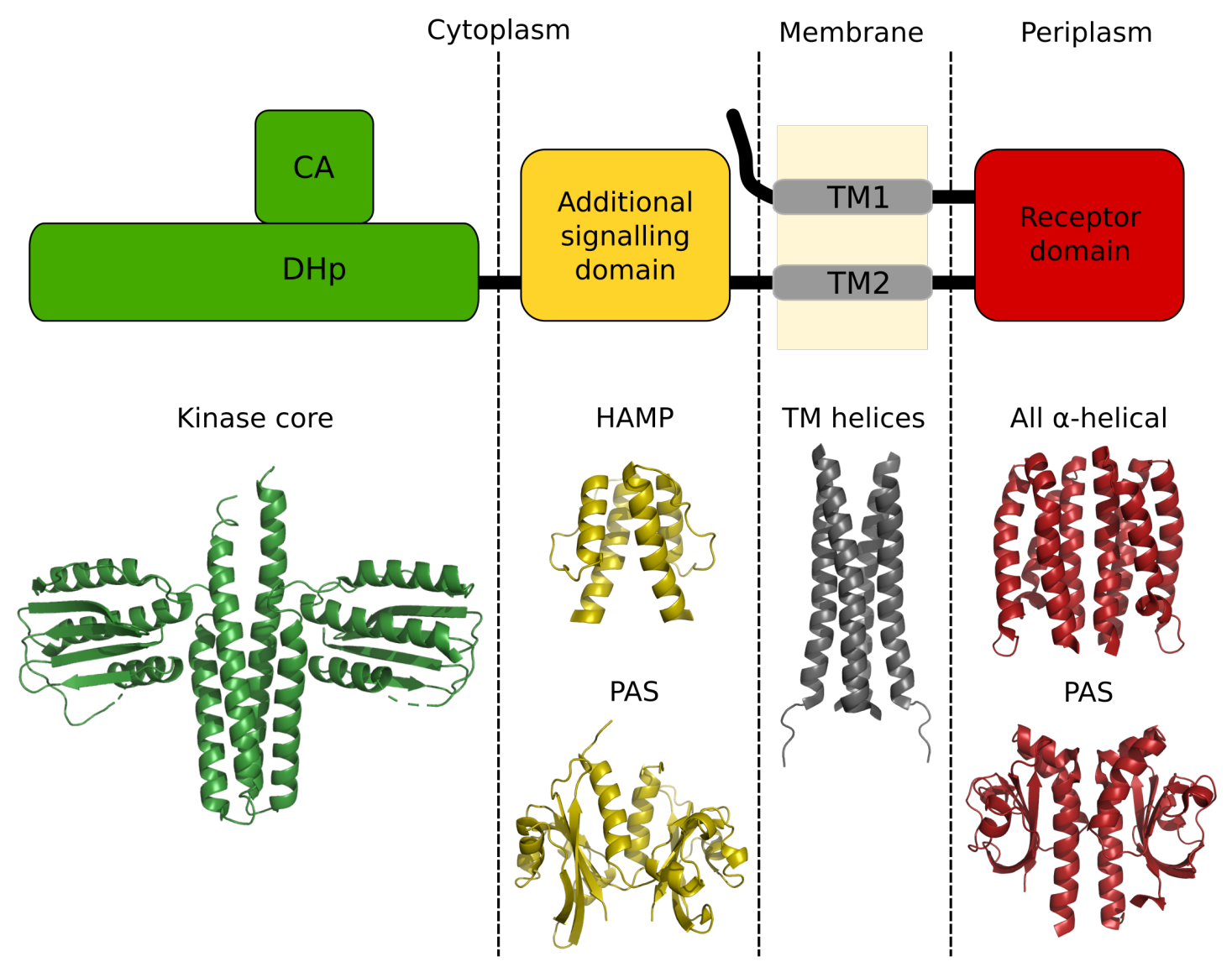

Figure 1.1: The general architecture of the monomeric unit of a HK together with some representative structure of the different domains. The functional state is a homodimer, as shown in the structures of the various domains in the bottom.

(Albanesi et al., 2009; Casino et al., 2009; Marina et al., 2005; Podgornaia et al., 2013; Wang et al., 2013), revealing details on structure and phosphotransfer mechanism.

The CA domain is a highly conserved $\alpha / \beta$ sandwich with three $\alpha$-helices packed against five anti-parallel $\beta$-strands. The ATP binds to this domain between two helices and is held by a loop also known as ATP-lid.

The DHp domain forms a homodimeric anti-parallel four-helical bundle. The catalytic histidine is located on the first $\alpha$-helix, at the beginning of a conserved seven amino-acids stretch (Bhate et al., 2015). The two helices of the DHp domain are connected by a hairpin loop which influences the handedness of the helix bundle in the DHp domain and the phosphorylation mechanism: cis autophosphorylation occurs with left-handed bundles, trans autophosphorylation with right-handed bundles (Ashenberg et al., 2013). Altering the length of the connecting hairpin 
loop affects the handedness and can inter-convert cis kinases to trans and viceversa (Casino et al., 2014). A relevant exception to this general rule is represented by the HK DesK which is believed to phosphorylate in trans, yet has a left-handed four-helix DHp bundle (Trajtenberg et al., 2010).

In addition to the kinase core, the other element that can be found in all the prototypical HKs is the receptor domain. For a membrane-bound HK with the simplest architecture, no receptor domain is present on the periplasmic side of the membrane and the adaptive response is initiated directly by the two transmembrane (TM) helices and the loop connecting them. This is the case for the thermosensor HK DesK, that uses the TM domain to detect differences in the membrane fluidity due to temperature changes (Cybulski et al., 2002).

However, the majority of HKs have one or more extra-cytoplasmic domain, that is able to sense a wide variety of signals and are responsible for the initial activation of the regulatory readout. Many structures of the sensing domains have been solved and molecular insights about signal detection and transmission can be gained from them.

The PAS (PER-ARNT-SIM) domain, also known as PDC (PhoQ-DcuS-CitA) domain, is perhaps the most common periplasmic sensing domain. In Klebsiella pneumoniae and Geobacillus thermodenitrificans CitA (Reinelt et al., 2003; Salvi et al., 2017; Sevvana et al., 2008) and in Escherichia coli DcuS (Cheung and Hendrickson, 2008; Pappalardo et al., 2003), the periplasmic PAS domain binds $\mathrm{C}_{4}$-dicarboxylate. In Salmonella typhymurium $\mathrm{PhoQ}$, the same domain is used to detect small antimicrobial peptides and $\mathrm{Mg}^{2+}$ ions using the membrane exposed surface. HKs with double-cache PAS domain have also been described. They comprise two consecutive periplasmic PAS domains, known as membrane distal and membrane proximal periplasmic PAS. The membrane distal periplasmic PAS domain can either bind its ligand, as in the case of DctB or KinD (Wu et al., 2013; Zhou et al., 2008), or it can be the interaction partner of other proteins, as in the case of LuxQ (Neiditch et al., 2006). The membrane proximal periplasmic PAS domain is less well studied. Nevertheless, it is reported that changes in the dimer interface of the membrane proximal periplasmic PAS domain of DctB are essential for the signalling relay (Liu et al., 2014).

Beside the PAS-like domains, all $\alpha$-helical domains are reported as extra-cytoplasmic sensor domain as well. They were first identified in the chemotaxis receptor Tar and Trs and known to bind amino acids (Milburn et al., 1991). A topologically similar domain has been described later in the HK NarQ, which binds directly 
nitrate or nitrite in a single binding site located at the dimer interface (Cheung and Hendrickson, 2009; Gushchin et al., 2017).

In addition to the kinase core and the extra-cytoplasmic sensor domain, many HKs present one or multiple additional cytosolic domains as direct C-terminal extension of the transmembrane helical elements. The cytosolic signalling domain can be either a PAS domain, like in CitA and in DcuS, or a HAMP domain (present in Histidine kinases, Adenylate cyclases, Methyl accepting proteins and Phosphatases). For the HAMP domain, several models of the signalling mechanism have been proposed. The conversion of piston-like shifts in the TM region to helical rotation output signal via tilting and lever like motions is confirmed by the only experimental structure available for the same domain in OFF and in the ON state (Gushchin et al., 2017).

Signal transduction of cytosolic PAS domain are less well studied and will be discussed in more detail in the section 1.5.

\subsubsection{Signalling mechanism}

Despite the wealth of biochemical data, structural mechanism of the TM signalling by HKs are poorly understood at the atomic level. Structural studies are hindered by the nature of the HKs, which are highly dynamic proteins and adopt multiple conformations to achieve the signal transduction. However, it is possible to speculate on plausible signalling mechanisms based on the evidences available for HK segments or just for isolated HK domains.

The first step in a prototypical HK is the relay of the signal from the receptor domain to the TM helices. Based on the structures available for isolated receptor domains in both ligand-free and ligand-bound state, a piston-like displacement of the C-termini of the receptor domain arises as a common mechanism. This has been reported for isolated all $\alpha$-helical sensors, such as the one of Tar (Milburn et al., 1991) and NarX (Cheung and Hendrickson, 2009). Piston-like motion, accompanied by helical rotations, was also reported in the context of the membrane by Gushchin et al. (2017), who studied a fragment of NarQ containing the two TM helices and the cytosolic signalling domain in addition to the receptor domain (Gushchin et al., 2017). In PAS domain receptors, contractions which could cause piston-like displacement have been observed in the isolated PASp domain (Sevvana et al., 2008) and in the context of the membrane fir a construct comprising the PASp, TM1, TM2 and PASc domain (see figure 1.2) (Salvi et al., 2017). 
Due to the great diversity of input signals and sensor domains, it can be questioned whether a common signal transmission is conserved across the different HKs. Based on the deposited structures, it was observed that most of the sensor domains share an extended helix at the dimer interface, termed p-helix. The phelix is proximal to the ligand-binding domain and connects the binding pocket to either TM1 or TM2. This might indicate that the ligand binding event exerts conformational changes on the p-helix which in turn effects the structure of the TM region (Bhate et al., 2015).

Structural information available about the TM signalling is scarce and most of the models are based on indirect data. For the four-helical bundle formed by dimeric HKs with two transmembrane helices, a great variety of collective motions is possible. The range of possibilities is greatly reduced if only symmetric displacements and skews are considered and only seven signalling models are left: piston-like, overall destabilization, dissociation, twist, skew, helical rotation, and diagonal scissoring (Gushchin and Gordeliy, 2018). Of these seven, only three were discussed as possible models for transmembrane signalling: piston, helical rotation and diagonal scissoring (Bhate et al., 2015).

The piston model has been demonstrated in Tar with cysteine cross-linking (Hughson and Hazelbauer, 1996) and repositioning of helix-flanking amino acids (Adase et al., 2012; Draheim et al., 2006; Miller and Falke, 2004). Besides Tar, piston-like displacement has been also shown for DcuS and CitA (Monzel and Unden, 2015; Salvi et al., 2017; Sevvana et al., 2008). The helical rotation model is based on observations made on LuxQ (Neiditch et al., 2006), DesK (Abriata et al., 2017) and AgrC Wang et al. (2014). Finally, diagonal scissoring was proposed for HK PhoQ based on cross-linking and molecular dynamics studies (Bhate et al., 2015; Molnar et al., 2014).

Of remarkable interest is the TM signalling mechanism in NarQ. For this HK, structural information is available directly from the crystal structure of the HK segment solved by Gushchin et al. (2017) for the symmetric apo (SA), for the symmetric holo (SH) and for the asymmetric holo (AH) state. Several conformational changes are revealed by the alignment of the SA with the SH state, with piston shifts happening near the cytoplasm-membrane interface, and diagonal scissoring and twisting at the periplasmic side. More complex conformational changes were revealed by the comparison of the $\mathrm{SH}$ and the $\mathrm{AH}$ states with pronounced skew and asymmetrical helical rotation (Gushchin et al., 2017). These conformational changes are often accompanied by kinks in the TM helical region positioned close 
to glycine or proline residues (Gushchin and Gordeliy, 2018).

After crossing the membrane, the input signal can be transmitted directly to the kinase core or to an intermediate cytosolic signalling domain. In the first case, the major common theme in signal transferring to the DHp and CA domains is the overall destabilization of the $\alpha$-helical linkers caused by helical rotations (Albanesi et al., 2009; Bhate et al., 2015; Hazelbauer et al., 2007; Parkinson et al., 2015; Saita et al., 2015; Wang et al., 2014). Therefore, this should be the output of the TM helices in proteins where they are directly followed by the DHp domain. In other proteins, the output signal is first handled by additional signalling domains, such as HAMP, PAS and GAF domains, which are found between TM and kinase core. Signal transduction of the cytosolic HAMP domain is well studied and several models have been proposed. In the dynamic bundles model, different signalling states differ in the overall stability of the domain (Parkinson, 2010; Sukomon et al., 2017; Zhou et al., 2009). The gearbox model suggest rearrangements of the coiled coil packing (Hulko et al., 2006, 2011). Other scissoring and pistoning models suggest that the relative orientation and position of the helices of the HAMP domain change during signalling (Airola et al., 2010; Gushchin et al., 2013; Wang et al., 2012). The latter models are all nicely illustrated on the NarQ structure solved by Gushchin et al. (2017), where gearbox-like changes and tilting convert the piston-like output of the TM helices into helical rotation (Gushchin et al., 2017).

Signal transduction of the cytosolic PAS domain is less well studied. A model based on overall destabilization has been proposed based on NMR and mutational studies (Etzkorn et al., 2008; Monzel et al., 2013; Salvi et al., 2017), but an atomic model of the signalling mechanism is still missing. Achieving better understanding and new atomic details of the signalling mechanism adopted by the cytosolic PAS domains is one of the main targets of this work.

\subsection{CitA family}

The CitA HK family is a good candidate for structural studies on these systems because it shows the most prevalent organization in HKs: an extra-cytosolic PAS sensor domain that is flanked by two TM helices, an additional PAS cytosolic signalling domain as C-terminal extension of the TM2 helix and a kinase core that includes the DHp and the CA domains. In addition to this, PAS domains are very 
well studied and widely adopted as receptor domains. In the last decades, two proteins of this family have been extensively studied: DcuS and CitA.

DcuS forms together with DcuR a TCS responsible for activation of dicarboxylate transport and fermentation as a response to the availability of aspartate, fumarate, malate and succinate (Golby et al., 1999; Zientz et al., 1998). DcuS binds $\mathrm{C}_{4}$-dicarboxylate in the periplasmic PAS domain and molecular insights of the rearrangements caused by the binding event can be gained from a liquid-state NMR-based model (Pappalardo et al., 2003) and from a X-ray crystal structure (Cheung and Hendrickson, 2008). Other biochemical assays shed light on the signalling mechanism of the cytoplasmic PAS domain and of the TM region (Monzel and Unden, 2015; Monzel et al., 2013) and showed that DcuS is constitutively ON in the absence of the dicarboxylate transporter DcuB or DctA (Steinmetz et al., 2014).

The citrate receptor CitA presents the same domain architecture of DcuS, with a periplasmic PAS (PASp) receptor domain flanked by two TM helices, an additional cytosolic PAS (PASc) domain and the kinase core, but active and the inactive state of CitA can be obtained both in vivo and in vitro, in contrast to DcuS. Together with CitB, CitA constitutes a TCS controlling the citrate transporter and fermentation (Bott et al., 1995). The ligand binding of CitA is highly selective towards citrate and does not recognize any other $\mathrm{C}_{4}$-dicarboxylate (Kaspar and Bott, 2002; Kaspar et al., 1999). From the available crystal structures of Klebsiella pneumoniae CitA periplasmic sensor domain (Reinelt et al., 2003; Sevvana et al., 2008), it is possible to understand the molecular mechanism of the binding event. The major structural rearrangement is the tightening of the $\beta$-sheet around the binding pocket, executing in turn a pull on the C-terminal $\beta$-strand and on the following TM2 helix. Based on this evidence, a piston-like motion has been proposed as the trigger for the transmembrane signalling (Sevvana et al., 2008).

Although the available crystal structures of the receptor domain of E. coli DcuS and of K. pneumoniae CitA represent a promising starting point for the understanding of the transmembrane signalling mechanism, structural studies of the 
membrane-embedded construct of the CitA-family HKs turned out to be a challenging task. Solid-state NMR (ssNMR) studies of E. coli DcuS were limited by the unavailability of the inactive state and of the PASc domain. The PASc domain is also a limiting factor for the studies on $K$. pneumoniae CitA as it resulted to be not stable neither for solution-state NMR nor for crystallization studies. E. coli CitA PASc was the first cytosolic signalling domain to be isolated and assigned via liquid-state NMR. However, also E. coli CitA samples were difficult to study as the PASc domain was invisible in the ssNMR experiments of a membraneembedded construct. To overcome this problem, CitA homologues were sought in thermophilic organisms, whose proteins are popular targets in structural biology due to their increased rigidity (Razvi and Scholtz, 2006). A CitA homologue was found in Geobacillus thermodenitrificans, a thermophilic organism characterised based on his genomic and and metabolic properties (White et al., 1993). By switching to $G$. thermodenitrificans CitA, it was possible not only to characterise the PASc domain in isolation via solution-state NMR and crystallography, but also to study it in both active and inactive state in the membrane-embedded construct via ssNMR.

\subsection{PAS domains}

PAS domains have been identified in proteins from all three kingdoms of life. They are signalling modules that monitor a great variety of changes in the surrounding environment of the cell, including light, redox potential, oxygen, small ligands, and overall energy level of a cell. PAS domains are combined with many different regulatory modules. As a consequence, a wide spectrum of cell regulatory responses are regulated by PAS-containing proteins.

Sequence homology among PAS domains is rather low. This is not surprising and follows their adoption in all kingdoms of life. For this reason, prediction of the PAS fold was made much easier after the development of algorithms like PSI BLAST (Altschul et al., 1990), that provide a way of detecting distant relationships between proteins. Indeed, several PAS domains have been identified only after the 3D structure was solved, e.g. the receptor domain of CitA, DcuS and PhoQ (Cho et al., 2006; Pappalardo et al., 2003; Reinelt et al., 2003). A prototypical PAS fold consists of an N-terminal helical element, a central $\beta$-sheet consisting of five anti-parallel strands and a loop that can have a varying number of short $\alpha$-helices 
opposite to the $\beta$-scaffold. The N-terminal helix is the element showing the largest variance both in terms of structure and of primary sequence (Taylor and Zhulin, 1999; Vreede et al., 2003). The extensive use of PAS domains as receptors is a consequence of the different mechanisms available for signal perception. Very often, small molecules are bound in the pocket created by the $\beta$-scaffold and the outer loop. This is the case for the HKs CitA (Kaspar et al., 1999; Reinelt et al., 2003; Sevvana et al., 2008), DcuS (Cheung and Hendrickson, 2008), and TodS (Lacal et al., 2006; Mosqueda et al., 1999). In other cases, PAS domains bind cofactors like flavine adenine dinucleotide (Key et al., 2007), heme (Gong et al., 2000) and Fe-S clusters (Müllner et al., 2008). A further mechanism for stimulus perception is the modification in the overall dynamics of the domain. This is the case of ArcB, where two intermolecular disulfide bridges are formed between the units of the homodimer in response to oxidative stress, leading to inactivation of the kinase (Malpica et al., 2004). Finally, a last mechanism is described for the PhoQ that is binding divalent cations at the interface between the membrane and the PAS domain to trigger different conformations (Cheung et al., 2008; Cho et al., 2006). Besides being employed as extra-cytosolic receptors, PAS domains also participate in signal transmission, like the additional cytosolic domain in HKs LuxQ (Neiditch et al., 2006) and CitA (Etzkorn et al., 2008). The non-ligand binding PAS domains are less well characterized as structural rearrangements cannot be triggered by ligand binding in the isolated domain and they need to be conducted on multi-domain kinases. DctB also presents a non-ligand binding PAS domain as a part of the double-cache periplasmic sensor domain (Zhou et al., 2008). In this case, rearrangements at the dimer interface of the membrane proximal PAS domain has been reported to be essential for the signalling transmission to the TM helices.

\subsection{NMR approach}

Histidine kinases are challenging systems for structural biology studies:

- Diffracting crystals of HKs that include the TM region are difficult to obtain without the employment of special approaches, such lipid cubic phase technologies (Cherozov, 2011) 
- Despite the remarkable progresses made by Cryo-EM, HKs are still too small to gain molecular insights of the signalling mechanism with this technique

- Liquid-state NMR is also affected by the size of the sample as it correlates with the molecular tumbling affecting the line-width and the spectral quality.

To overcome the limitations of these techniques alone, a combined approach similar to the one already described by Etzkorn et al. (2008) has been used throughout this work.

The HK Citrate receptor A was selected to investigate the transmembrane signalling mechanism of the HKs for various reasons: first of all, the domain organization is the most common among the HKs, with the extra-cytosolic receptor domain flanked by two TM helices and a membrane-bound dimeric functional state. Second, the ligand of CitA and the binding pocket is well characterised and both signalling states of the receptor are accessible in vivo as well as in vitro. Nevertheless, the signalling mechanism of the transmembrane helices and the role of the additional cytosolic PAS domain are not clear yet and their understanding in the CitA model system is one of the key questions of this work.

The focus of this study is on the signal transmission across the membrane, therefore the DHp and CA domains are absent in all the studied constructs of CitA. This reduces the molecular weight from $59 \mathrm{kDa}$ to $34 \mathrm{kDa}$ per monomer. This shortened construct is termed CitApc or PASpc and has been used in ssNMR spectroscopy after reconstitution in liposomes (see figure 1.2).

To aid in the assignment and analysis of the ssNMR spectra, the PASp and PASc were also produced as isolated, soluble proteins. These can be assigned using liquid-state NMR. If no major structural differences are present between the soluble domains and the transmembrane construct PASpc, the results of the isolated PAS domains can be used to predict the resonances values of CitApc and to facilitate the assignment if the transmembrane construct, as shown in figure 1.3. The only resonances that can not be assigned based on the assignment of the isolated PASp and PASc are the ones of the TM helices, which constitute a small subset of the whole spectrum and can be assigned using a de novo sequential assignment based on 3D ssNMR experiments. 


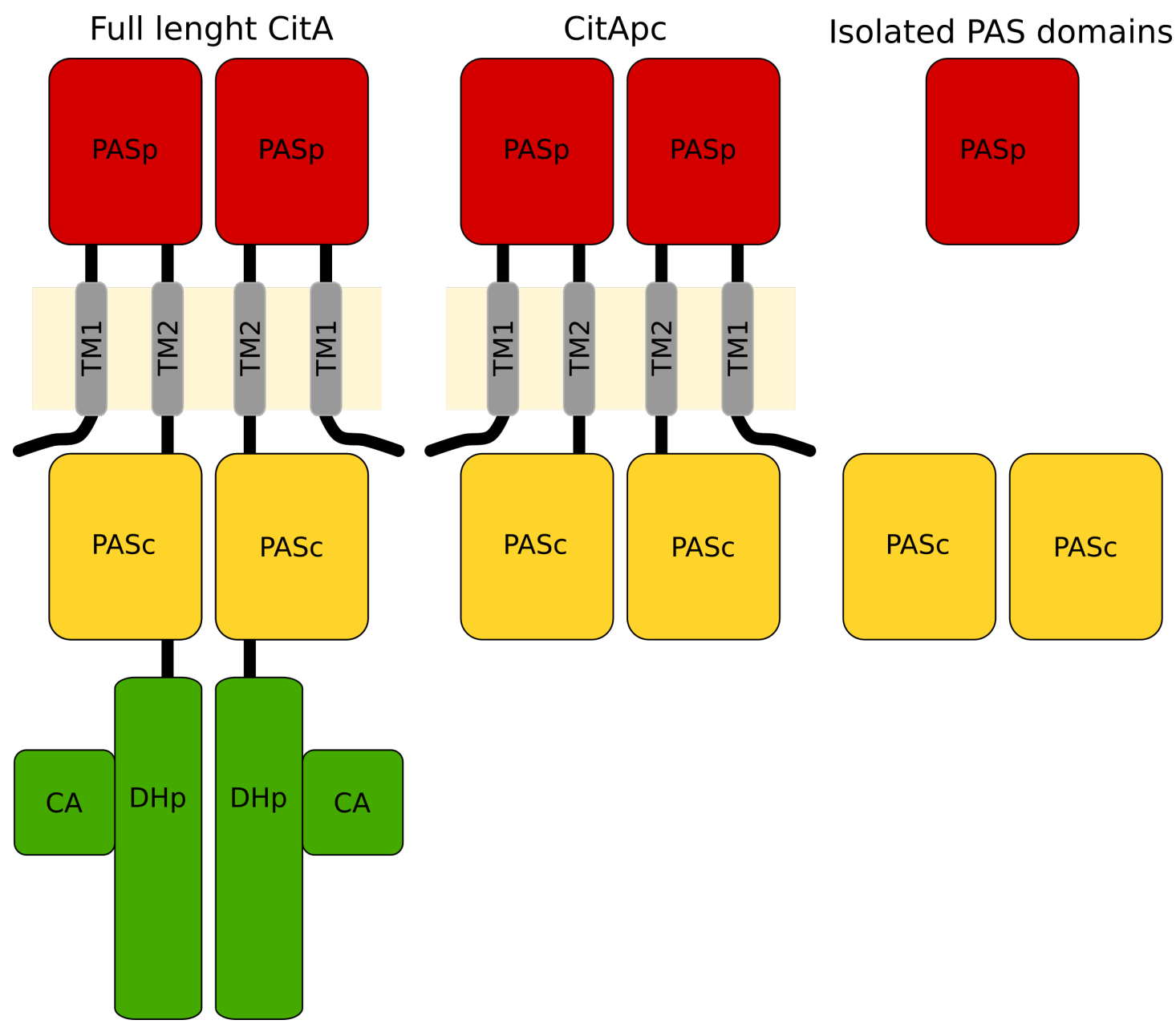

Figure 1.2: An overview of some possible constructs of $G$. thermodenitrificans CitA. In this work the so-called CitApc (middle) has been used for ssNMR experiments. The isolated domains were assigned via liquid-state NMR and used to facilitate the assignment of ${ }^{1} \mathrm{H}$ and ${ }^{13} \mathrm{C}$ detected ssNMR spectra.

In addition to the prediction of resonance values, liquid-state NMR was also employed to study dynamic and chemical exchange properties of the isolated domains as these features could also be relevant to the signalling.

Finally, ${ }^{1} \mathrm{H}$-detected ssNMR has been also exploited in this work to characterise the transmembrane signalling in the CitApc construct. All the results obtained via ssNMR on HKs were exclusively based on ${ }^{13} \mathrm{C}$-detected experiments (Etzkorn et al., 2008; Salvi et al., 2017; Schomburg, 2014). ${ }^{1} \mathrm{H}$ detection in magic-angle spinning (MAS) NMR emerged as a new, powerful tool for membrane proteins thanks to the development of very fast (60 kHz and above) spinning probes and higher magnetic fields, that together are responsible for reducing the ${ }^{1} \mathrm{H}^{-}{ }^{1} \mathrm{H}$ dipolar interactions and improving resolution in the ssNMR spectra (Schubeis et al., 2017). This approach presents different advantages over the ${ }^{13} \mathrm{C}$-detected MAS 


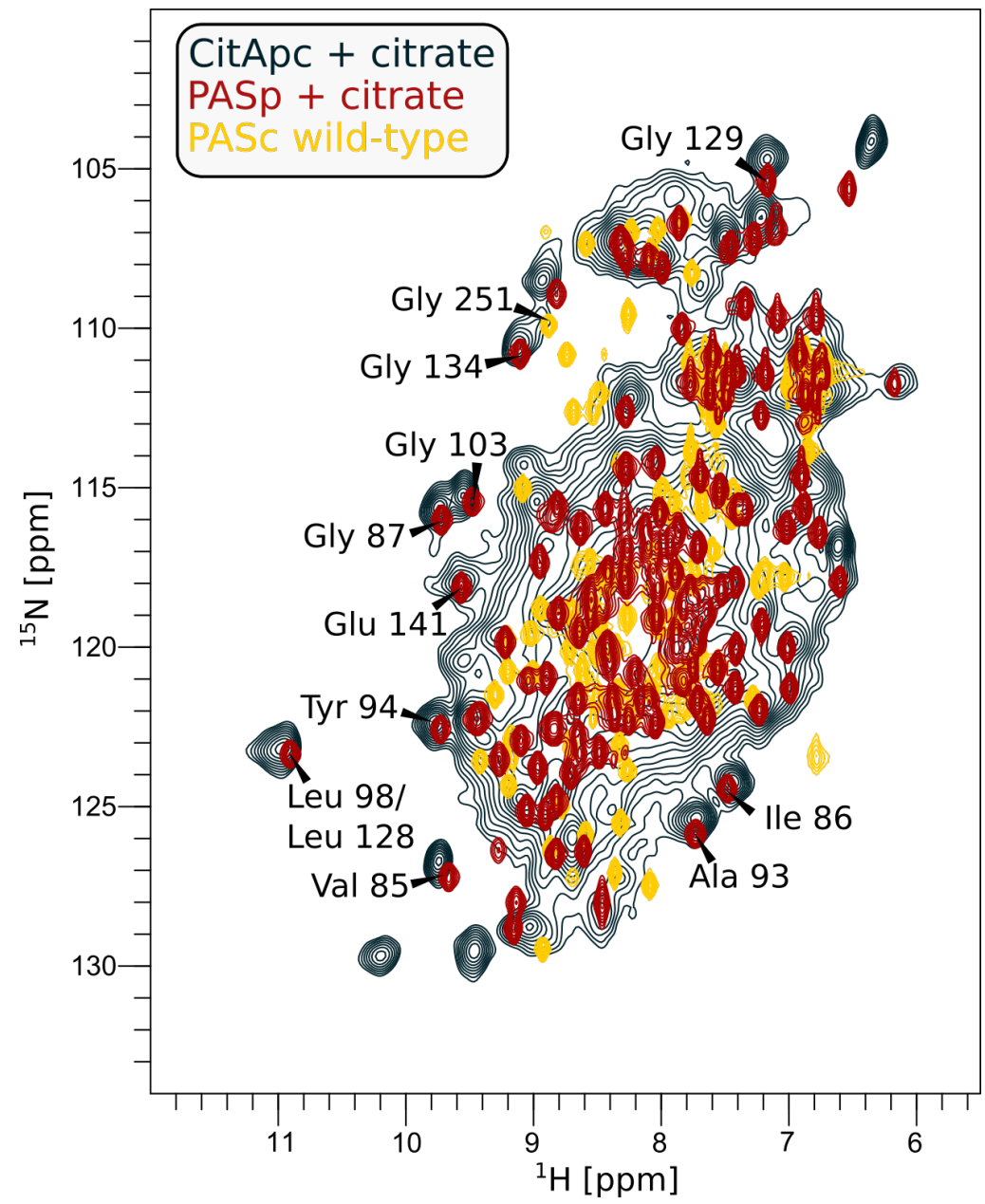

Figure 1.3: Overlap of the ssNMR hNH spectra of citrate-bound CitApc C12AR93A (dark blue) with the liquid-state ${ }^{15} \mathrm{~N}-\mathrm{HSQC}$ of citrate-bound PASp (in red) and the ${ }^{15} \mathrm{~N}-\mathrm{HSQC}$ of PASc wild-type (in yellow). The assignment for the representative, isolated cross-peaks is reported to illustrate the adopted approach. The experimental conditions are reported in table 2.4 for the hNH and in table 2.2 and 2.1 for the ${ }^{15} \mathrm{~N}-\mathrm{HSQC}$.

NMR. First, smaller rotors are needed to achieve higher spinning frequency, leading to the reduction of about one magnitude of the amount of sample needed. However, the low amount of protein present in the rotor does not affect the NMR signal intensity as it is compensated by the four fold higher gyromagnetic ratio of ${ }^{1} \mathrm{H}$ compared to ${ }^{13} \mathrm{C}$ and by longer coherence lifetimes. All included, time and costs for screening of new sample conditions or new protein preps are significantly reduced. Second, the proton network of a protein contains biologically relevant informations that are not measured directly with ${ }^{13} \mathrm{C}$-detected MAS NMR or are lost in perdeuterated samples. Thus, proton detected MAS NMR of fully protonated proteins at spinning speed above $100 \mathrm{kHz}$ is more effective when it comes to understanding of molecular mechanism. Third, 3D solid-state and liquid-state 
NMR experiments share all the dimensions. Thus, the chemical shifts of CitApc does have to be predicted from the ones of the isolated PAS domains as solid-state and liquid-state spectra can be directly overlapped and compared. This allows us to detect both similarities and differences in a more practical way.

\subsubsection{Other biophysical techniques}

To further facilitate the interpretation of the NMR data, results obtained using other biophysical techniques are also presented and discussed in this work. In vivo assays were performed in the lab of Prof. Gottfried Unden (Institute of Molecular Physiology, Johannes Gutenberg-University of Mainz, Mainz, GER) to analyse the effects of mutations on the functionality of CitA.

Crystal structures were solved by Dr. Stefan Becker (Department of NMR-based Structural Biology, Max-Planck-Institute for Biophysical Chemistry, Göttingen, GER) and provided high resolution data for the isolated domains.

Cryogenic Optical Localization in 3D (COLD) (Weisenburger et al., 2017) experiments in collaboration with Prof. Vahid Sandoghdar (Max Planck Institute for the Science of Light, Erlangen, GER) were used to measure inter-unit distances of the soluble PASc homo-dimers. 


\section{Material and Methods}

\section{$2.1 \quad$ Liquid- state NMR experiments}

\subsubsection{Sample preparation}

Samples of the soluble periplasmic and cytosolic PAS domains were used for liquidstate NMR experiments.

PASp R93A samples consisted of ${ }^{13} \mathrm{C}$ and ${ }^{15} \mathrm{~N}$ labelled protein in $20 \mathrm{mM}$ sodium phosphate (pH 6.5) and 90/10\% $\mathrm{H}_{2} \mathrm{O} / \mathrm{D}_{2} \mathrm{O}$. Citrate was added to the buffer with a concentration of $5 \mathrm{mM}$ to obtain the bound state of the PASp.

PASc wild-type, PASc N288D and PASc R289D samples consisted of ${ }^{13} \mathrm{C}$ and ${ }^{15} \mathrm{~N}$ labelled proteins in $20 \mathrm{mM}$ sodium phosphate $(\mathrm{pH} 6.5)$ and $90 / 10 \% \mathrm{H}_{2} \mathrm{O} / \mathrm{D}_{2} \mathrm{O}$. A further sample was produced for the PASc wild-type mixing ${ }^{13} \mathrm{C}$ labelled protein with ${ }^{15} \mathrm{~N}$ labelled protein in a 1:1 ratio. The same procedure was also carried for the PASc N288D and PASc R289D mutants.

All the samples were expressed and purified as described in Salvi et al. (2017) and kindly provided by Karin Giller and Dr. Stefan Becker (Department of NMR-based Structural Biology, Max-Planck-Institute for Biophysical Chemistry, Göttingen, GER))

\subsubsection{General procedures}

Liquid-state NMR experiments were recorded using $5 \mathrm{~mm}$ Shigemi tubes (Shigemi Inc., Allison Park, USA), $5 \mathrm{~mm}$ and $3 \mathrm{~mm}$ NMR tubes from Sigma-Aldrich and 5 $\mathrm{mm}$ and $3 \mathrm{~mm}$ tubes from Hilgenberg (Hilgenberg GmbH, Malsfeld, Germany) at a protein concentration between 1.4 and $2.0 \mathrm{mM}$. All the spectra were acquired at a temperature of $25^{\circ}$ on Bruker spectrometers and processed using Bruker Topspin 3.2 or NMRpipe (Delaglio et al., 1995). Backbone and side-chain assignments were carried out using the CcpNMR analysis software (Vranken et al., 2005). A summary of the experiments recorded is given in the tables 2.1 and 2.2 


\begin{tabular}{|c|c|c|}
\hline \multicolumn{3}{|c|}{ Gt CitA PASp R93A } \\
\hline Spectrum & Field $[\mathrm{MHz}]$ & Experimental time \\
\hline${ }^{15} \mathrm{~N}-\mathrm{HSQC}$ & 800 & $20 \mathrm{~m}$ \\
\hline${ }^{15} \mathrm{~N}-\mathrm{HSQC}-\mathrm{NOESY}(80 \mathrm{~ms})$ & 900 & $3 \mathrm{~d}$ \\
\hline${ }^{13} \mathrm{C}-\mathrm{HSQC}-\mathrm{NOESY}(100 \mathrm{~ms})$ & 800 & $4 \mathrm{~d} 21 \mathrm{~h}$ \\
\hline \multicolumn{3}{|c|}{ Gt CitA PASp R93A + citrate } \\
\hline Spectrum & Field $[\mathrm{MHz}]$ & Experimental time \\
\hline${ }^{15} \mathrm{~N}-\mathrm{HSQC}$ & 700 & $20 \mathrm{~m}$ \\
\hline${ }^{15} \mathrm{~N}-\mathrm{HSQC}-\mathrm{NOESY}(80 \mathrm{~ms})$ & 900 & $2 \mathrm{~d} 2 \mathrm{~h}$ \\
\hline${ }^{13} \mathrm{C}-\mathrm{HSQC}-\mathrm{NOESY}(100 \mathrm{~ms})$ & 700 & $3 \mathrm{~d} 17 \mathrm{~h}$ \\
\hline
\end{tabular}

Table 2.1: Liquid-state NMR experiments recorded for the soluble PASp R93A with and without citrate. In brackets are the mixing times used for the NOESY experiments. All magnets were equipped with a $5 \mathrm{~mm}$ triple-resonance cryogenic probe

\subsection{3 $\quad{ }^{15} \mathrm{~N}$ CEST experiments}

${ }^{15} \mathrm{~N}$ CEST experiment were recorded as described by Vallurupalli et al. (Vallurupalli et al., 2017). ${ }^{15} \mathrm{~N}$ radio-frequency field strengths of 20 and $80 \mathrm{~Hz}$ were applied together with ${ }^{1} \mathrm{H}$ decoupling of $3.5 \mathrm{kHz}$ during a relaxation delay of $400 \mathrm{~ms}$. CEST data consisted of a series of 2D spectra, acquired in an interleaved fashion, corresponding to ${ }^{15} \mathrm{~N}$ irradiation offsets incremented in steps of $0.5 \mathrm{ppm}$. Additionally, a reference experiment for which the relaxation delay is set to 0 was recorded.

\subsubsection{Amide exchange patterns}

To analyse the amide exchange patterns of the $G$. thermodenitrificans CitA PASc wild-type and of the N288D and R289D mutants, protein samples in $20 \mathrm{mM}$ sodium phosphate buffer at $\mathrm{pH} 6.5$ were dialysed for two days against $20 \mathrm{mM}$ sodium phosphate deuterated buffer at same $\mathrm{pH}$. Experiments were recorded within 6 hours after the end of the dialysis to avoid any problem of $\mathrm{pH}$ stability. The signal intensities of the ${ }^{15} \mathrm{~N}-\mathrm{HSQC}$ of samples in deuterated buffer were compared signal intensities of the reference ${ }^{15} \mathrm{~N}-\mathrm{HSQC}$ spectrum of samples in $\mathrm{H}_{2} \mathrm{O}$ buffer. 


\begin{tabular}{|c|c|c|}
\hline \multicolumn{3}{|c|}{ Gt CitA PASc wild-type } \\
\hline Spectrum & Field $[\mathrm{MHz}]$ & Experimental time \\
\hline${ }^{15} \mathrm{~N}-\mathrm{HSQC}$ & 800 & $20 \mathrm{~m}$ \\
\hline HNCA & 900 & $1 \mathrm{~d} 21 \mathrm{~h}$ \\
\hline CBCAcoNH & 900 & $2 \mathrm{~d} 15 \mathrm{~h}$ \\
\hline $\mathrm{HNCO}$ & 800 & $15 \mathrm{~h}$ \\
\hline HCCH-TOCSY & 600 & $2 \mathrm{~d} 20 \mathrm{~h}$ \\
\hline${ }^{15} \mathrm{~N}-\mathrm{HSQC}-\mathrm{NOESY}(80 \mathrm{~ms})$ & 800 & $3 \mathrm{~d} 6 \mathrm{~h}$ \\
\hline${ }^{13} \mathrm{C}-\mathrm{HSQC}-\mathrm{NOESY}(100 \mathrm{~ms})$ & 800 & $6 \mathrm{~d} 15 \mathrm{~h}$ \\
\hline${ }^{13} \mathrm{C}-{ }^{15} \mathrm{~N}$ Double-edited NOESY (250 ms) & 950 & $3 \mathrm{~d} 5 \mathrm{~h}$ \\
\hline \multicolumn{3}{|c|}{ Gt CitA PASc R289D } \\
\hline Spectrum & Field $[\mathrm{MHz}]$ & Experimental time \\
\hline${ }^{15} \mathrm{~N}-\mathrm{HSQC}$ & 800 & $20 \mathrm{~m}$ \\
\hline $\mathrm{HNCA}$ & 600 & $1 \mathrm{~d} 20 \mathrm{~h}$ \\
\hline CBCAcoNH & 600 & $2 \mathrm{~d} 12 \mathrm{~h}$ \\
\hline $\mathrm{HNCO}$ & 600 & $15 \mathrm{~h}$ \\
\hline HCCH-TOCSY & 700 & $2 \mathrm{~d} 23 \mathrm{~h}$ \\
\hline${ }^{15} \mathrm{~N}-\mathrm{HSQC}-\mathrm{NOESY}(80 \mathrm{~ms})$ & 800 & $2 \mathrm{~d} 23 \mathrm{~h}$ \\
\hline${ }^{13} \mathrm{C}-\mathrm{HSQC}-\mathrm{NOESY}$ (100 ms) & 800 & $4 \mathrm{~d} 1 \mathrm{~h}$ \\
\hline${ }^{13} \mathrm{C}-{ }^{15} \mathrm{~N}$ Double-edited NOESY (250 ms) & 950 & $2 \mathrm{~d} 20 \mathrm{~h}$ \\
\hline \multicolumn{3}{|c|}{ Gt CitA PASc N288D } \\
\hline Spectrum & Field $[\mathrm{MHz}]$ & Experimental time \\
\hline${ }^{15} \mathrm{~N}-\mathrm{HSQC}$ & 800 & $20 \mathrm{~m}$ \\
\hline $\mathrm{HNCA}$ & 600 & $1 \mathrm{~d} 22 \mathrm{~h}$ \\
\hline CBCAcoNH & 600 & $2 \mathrm{~d} 17 \mathrm{~h}$ \\
\hline $\mathrm{HNCO}$ & 600 & $17 \mathrm{~h}$ \\
\hline HCCH-TOCSY & 600 & $4 \mathrm{~d} 4 \mathrm{~h}$ \\
\hline${ }^{15} \mathrm{~N}-\mathrm{HSQC}-\mathrm{NOESY}(80 \mathrm{~ms})$ & 800 & $3 \mathrm{~d} 3 \mathrm{~h}$ \\
\hline${ }^{13} \mathrm{C}-\mathrm{HSQC}-\mathrm{NOESY}$ (100 ms) & 800 & $3 \mathrm{~d} 20 \mathrm{~h}$ \\
\hline${ }^{13} \mathrm{C}-{ }^{15} \mathrm{~N}$ Double-edited NOESY (250 ms) & 950 & $2 \mathrm{~d} 22 \mathrm{~h}$ \\
\hline
\end{tabular}

Table 2.2: Liquid-state NMR experiments recorded for the soluble PASc wild-type and N288D and R289D mutants. In brackets the mixing times used for the NOESY experiments. All the spectrometers were equipped with a 5 $\mathrm{mm}$ triple-resonance cryogenic probe.

\subsection{Secondary chemical shift analysis}

The relationship between chemical shifts and protein secondary structure has been used from the early 90's (Wishart et al., 1991). In this work, $\mathrm{C}_{\alpha}$ and $\mathrm{C}_{\beta}$ chemical shifts were used in combinations to determine the local secondary chemical shift $\left(\delta_{s c s}\right)$ subtracting the random coil chemical shifts $\left(\delta_{c o i l}\right)$ from the experimental values $\left(\delta_{\text {exp }}\right)$ :

$$
\delta_{s c s}=\delta_{\text {exp }}-\delta_{\text {coil }}
$$


Furthermore, the $\delta_{s c s}$ obtained for $\mathrm{C}_{\beta}$ was subtracted from the one obtained for $\mathrm{C}_{\alpha}$, as these experience opposite dependence on the secondary structure.

With this notation, negative values correspond to $\beta$-strand structures while positive values reflect helical elements. The secondary chemical shifts were evaluated using the random coil chemical shifts proposed by Wang and Jardetzky (Wang and Jardetzky, 2002).

\section{$2.3 \quad$ Solid-state NMR experiments}

\subsubsection{Sample preparation}

Samples of the CitApc construct reconstituted in liposomes were used for ssNMR experiments. CitApc R93A samples used for ${ }^{13} \mathrm{C}$ detected experiments consisted of ${ }^{13} \mathrm{C}$ and ${ }^{15} \mathrm{~N}$ labelled protein in $20 \mathrm{mM}$ Tris- $\mathrm{HCl}(\mathrm{pH} 7.4)$ embedded in pure DMPC liposomes. Citrate was added to the buffer with in a $5 \mathrm{mM}$ concentration to obtain the bound state.

For sample optimization, ${ }^{13} \mathrm{C}$ and ${ }^{15} \mathrm{~N}$ labelled samples of CitApc $\mathrm{C} 12 \mathrm{~A}$ in the bound state were tested with different lipids and buffer compositions (see table 3.4). After analysis of ${ }^{15} \mathrm{~N}$ line-width and visibility in the $3 \mathrm{D}$ hCANH, the best spectral quality was achieved using $20 \mathrm{mM}$ sodium phosphate ( $\mathrm{pH} 6.5)$ buffer and a 9:1 of 1,2-dimyristoyl-sn-glycero-3-phosphocholine (DMPC) and 1,2-dimyristoylsn-glycero-3-phosphate (DMPA) ratio for the liposomes. These conditions were also used to produce ${ }^{13} \mathrm{C},{ }^{15} \mathrm{~N}$ and ${ }^{2} \mathrm{H}$ labelled samples of CitApc C12AR93A for the ${ }^{1} \mathrm{H}$ detected experiments.

All the samples were expressed and purified as described in (Salvi et al., 2017) and kindly provided by Karin Giller and Dr. Stefan Becker (Department of NMR-based Structural Biology, Max-Planck-Institute for Biophysical Chemistry, Göttingen, GER)).

\subsubsection{General procedures}

All samples were packed in ssNMR rotors from Bruker. The rotors with 1.3 $\mathrm{mm}$ diameter were filled using the spiNpack tool from Giotto Biotech (Giotto 
Biotech S.r.l., Sesto Fiorentino, Italy). For signal referencing, DSS (4,4-dimethyl4-silapentane-1-sulfonic acid) was added to the buffer at concentrations between $0.5 \%$ and $1 \%(\mathrm{w} / \mathrm{v})$. The temperature of the spinning sample was calculated from the chemical shift of bulk water. ${ }^{13} \mathrm{C}$ detected spectra were acquired between $7^{\circ}$ and $10^{\circ} \mathrm{C}$, while ${ }^{1} \mathrm{H}$ detected experiments were recorded at $25^{\circ} \mathrm{C}$.

\subsection{3 ${ }^{13} \mathrm{C}$ detected spectra}

In the attempt to reduce spectral crowding and isolate the signal from the TM regions, $3.2 \mathrm{~mm}$ rotors packed with CitApc samples were soaked in $\mathrm{D}_{2} \mathrm{O}$ buffer for two weeks. Due to the strong reduction of the overall NMR signal, it was not possible to acquire a set of triple resonance experiments for sequential assignment. The final spectra were obtained by adding several blocks of the same experiment to improve the signal-to-noise ratio. The $2 \mathrm{D} \mathrm{hNCA}$ and $3 \mathrm{D}$ hNCACB spectra were analysed based on the assignment already published (Salvi et al., 2017). A summary of the experiments is reported in table 2.3 .

\begin{tabular}{lccc}
\hline \multicolumn{4}{c}{ Gt CitApc R93A } \\
\hline Spectrum & Experimental time & no. of blocks & Spinning speed $[\mathrm{kHz}]$ \\
\hline hNCA & $24 \mathrm{~h} 31 \mathrm{~m}$ & 4 & 21 \\
hNCACB & $23 \mathrm{~h} 58 \mathrm{~m}$ & 10 & 21 \\
\hline \multicolumn{4}{c}{ Gt CitApc R93A + citrate } \\
\hline Spectrum & Experimental time & no. of blocks & Spinning speed $[\mathrm{kHz}]$ \\
\hline hNCA & $23 \mathrm{~h} 30 \mathrm{~m}$ & 3 & 21 \\
hNCACB & $23 \mathrm{~h} 28 \mathrm{~m}$ & 8 & 21 \\
\hline
\end{tabular}

Table 2.3: Solid-state NMR experiments acquired for CitApc R93A in $\mathrm{D}_{2} \mathrm{O}$ buffer with and without citrate. All the spectra were recorded on a Bruker 850 $\mathrm{MHz}$ spectrometer equipped with a triple channel $3.2 \mathrm{~mm}$ MAS probe. In the table is reported the experimental time of a single block.

\subsection{4 ${ }^{1} \mathbf{H}$ detected spectra}

Resonance assignment was revolutionised by the availability of fast MAS probes and new, robust methodologies were developed (Barbet-Massin et al., 2014; Fricke et al., 2017). In particular, a set of six 3D correlations has been proposed, where the intra-residue correlation provided by the hCONH and hCANH spectra are complemented by additional experiments which include $\mathrm{C}-\mathrm{C}$ coherence transfer to achieve the inter-residue correlation (Barbet-Massin et al., 2014). The complete 
set of six experiments was recorded for the CitApc C12AR93A sample in the bound state and the experimental details are summarized in table 2.4. The final spectra were obtained by adding several blocks of the same experiment to improve the signal-to-noise ratio.

\begin{tabular}{lccc}
\hline \multicolumn{4}{c}{ Gt CitApc C12AR93A + citrate } \\
\hline Spectrum & Experimental time & no. of blocks & Spinning speed $[\mathrm{kHz}]$ \\
\hline hNH & $15 \mathrm{~h} 17 \mathrm{~m}$ & 1 & 55 \\
hCANH & $23 \mathrm{~h} 57 \mathrm{~m}$ & 2 & 55 \\
hcaCOcaNH & $22 \mathrm{~h} 37 \mathrm{~m}$ & 9 & 55 \\
hCONH & $23 \mathrm{~h} 37 \mathrm{~m}$ & 2 & 55 \\
hcaCOcaNH & $23 \mathrm{~h} 34 \mathrm{~m}$ & 9 & 55 \\
hcaCBcaNH & $1 \mathrm{~d} 23 \mathrm{~h} 45 \mathrm{~m}$ & 6 & 55 \\
hcaCBcocaNH & 1d $23 \mathrm{~h} 56 \mathrm{~m}$ & 7 & 55 \\
\hline
\end{tabular}

Table 2.4: Solid-state NMR experiments acquired for the ${ }^{1} \mathrm{H}$-detection based assignment of CitApc C12AR93A with citrate]. All the spectra were recorded on a Bruker $850 \mathrm{MHz}$ spectrometer equipped with a four channel $1.3 \mathrm{~mm}$ MAS probe. In the table is reported the experimental time of a single block.

\subsection{Solvent PRE}

NMR spectroscopy gained popularity in the study of protein dynamics due to its capability to investigate motional time-scales ranging from the picoseconds to the seconds with atomic resolution. Briefly, the intrinsic nuclear spin relaxation resulting from the time-dependent stochastic modulation of dipole-dipole interactions and electronic environment surrounding the nuclei is influenced by fast internal motions occurring on time-scales up to the overall correlation time $\left(\tau_{c}\right.$, usually in the order of nanoseconds) of the molecule in solution and longitudinal and transverse relaxation rates $\left(R_{1}\right.$ and $R_{2}$, respectively) can be exploited to probe this dynamic range.

For the idealized random field mechanism, $R_{1}$ and $R_{2}$ are described by the equations:

$$
\begin{gathered}
R_{1}=\frac{1}{T_{1}}=\gamma^{2}\left\langle B^{2}\right\rangle J\left(\omega_{0}\right) \\
R_{2}=\frac{1}{T_{2}}=\frac{1}{2} \gamma^{2}\left\langle B^{2}\right\rangle J\left(\omega_{0}\right)+\frac{1}{2} \gamma^{2}\left\langle B^{2}\right\rangle J(0)
\end{gathered}
$$

where $\left\langle B^{2}\right\rangle$ is the mean square value of the field causing the relaxation, $\gamma$ is the gyromagnetic ratio of the two interacting spins and $J(\omega)$ is the spectral density describing the correlation time $\tau_{c}$. 
The presence of an unpaired electron spin affects to a great extent both $R_{1}$ and $R_{2}$ due its interaction with a nuclear spin. Molecules that bear an unpaired electron spin can be covalently bound to the NMR sample or solubilized in the buffer. The former method is exploited to obtain intra and inter molecule distances, while the latter proved itself very useful to to identify solvent-exposed regions of a protein and is known as Solvent Paramagnetic Relaxation Enhancement (sPRE).

Pintacuda and Otting (2002) described the effect of a soluble paramagnetic agent on ubiquitin with a "second sphere model", where the paramagnetic relaxation agent is assumed to form a non-specific, yet rotationally correlated complex with the protein. In this description, the dipolar interaction between the electron spin $\mathrm{S}$ and the nuclear spin I is modulated by the molecular rotation of the protein (correlation time $\tau_{R}$ ), the electron relaxation $\left(\mathrm{T}_{1 e}\right)$ and the lifetime of the intermolecular adduct $\left(\tau_{M}\right)$ (Banci et al., 1991; Peters et al., 1996). The $\mathrm{T}_{1, p}$ and $\mathrm{T}_{2, p}$ relaxation times of the nuclear spin $\mathrm{I}$ in a paramagnetic sample are given by:

$$
\begin{array}{r}
\frac{1}{T_{1, p}}=\frac{2}{15}\left(\frac{\mu_{0}}{4 \pi}\right)^{2} \frac{\gamma_{I}^{2}\left(g_{J} \mu_{B}\right)^{2} J(J+1)}{r^{6}}\left(\frac{3 \tau_{c}}{1+\omega_{I}^{2} \tau_{c}^{2}}+\frac{7 \tau_{c}}{1+\omega_{S}^{2} \tau_{c}^{2}}\right) \\
\frac{1}{T_{2, p}}=\frac{1}{15}\left(\frac{\mu_{0}}{4 \pi}\right)^{2} \frac{\gamma_{I}^{2}\left(g_{J} \mu_{B}\right)^{2} J(J+1)}{r^{6}}\left(4 \tau_{c}+\frac{3 \tau_{c}}{1+\omega_{I}^{2} \tau_{c}^{2}}+\frac{13 \tau_{c}}{1+\omega_{S}^{2} \tau_{c}^{2}}\right)
\end{array}
$$

where $\mu_{0}$ is the induction constant, $\gamma_{I}$ is the gyromagnetic ratio of the nuclei I, $g_{j}$ is the Landé factor, $\mu_{B}$ is the Bohr magneton, $r$ is the distance between the electron and the nuclear spin and $\omega_{I}$ and $\omega_{S}$ are the Larmor frequency of the nuclei I and of the electron, respectively. The correlation time $\tau_{c}$ is given by:

$$
\frac{1}{\tau_{c}}=\frac{1}{T_{1 e}}+\frac{1}{\tau_{M}}+\frac{1}{\tau_{r}}
$$

The effective distance $r$ can be determined using a grid search on a structural model to predict the relaxation rates (Hartlmüller et al., 2017; Pintacuda and Otting, 2002). In this work, the algorithm developed by Hartlmüller et al. was used to predict the theoretical values. Its output is the slope of the linear dependence of $R_{1}$ in function of the paramagnetic agent concentration. Thus, high values are predicted for spins close to the protein surface and low values for spins far away from the surface.

The same quantity can be measured experimentally by evaluating $R_{1}$ at different concentrations of the paramagnetic agent to gain insights about intermolecular interactions comparing the experimental with the theoretical values (Hartlmüller et al., 2017; Öster et al., 2017). 
Another method to measure experimentally the effect of a paramagnetic agent on the relaxation properties of a nuclear spin is to analyse the intensity of the NMR signal. Considering that the peak line-width is proportional to the transverse relaxation rate $R_{2}$, the signal of a nuclear spin located on the protein surface, and thus close to the unpaired electron spin, is significantly broader compared to a nuclear spin embedded in the core of the protein, and thus far from the paramagnetic agent. The perturbations on $\mathrm{R}_{2}$ also have an impact on peak heights, which can be readily estimated from any spectrum. Monitoring peak intensities of a $1 \mathrm{D}$ NMR spectrum in function of the paramagnetic dopant agent also results in a linear correlation. Unfortunately, 1D NMR experiments are not suitable to study residue specific properties of a protein and, depending on chemical shift dispersion and size of the sample, at least 2D spectra are needed. In the case of a ${ }^{15} \mathrm{~N}-\mathrm{HSQC}$, the contribution of ${ }^{1} \mathrm{H}$ and ${ }^{15} \mathrm{~N}$ gives rise to the following quadratic dependence:

$$
s P R E_{R_{2}}=\left(R_{2_{H, 0}}+s P R E_{H} C\right)\left(R_{2_{N, 0}}+s P R E_{N} C\right)
$$

where $C$ is the concentration of the paramagnetic agent, $R_{2_{H, 0}}$ and $R_{2_{N, 0}}$ are the transverse relaxation rates of proton and nitrogen in the diamagnetic sample, $s P R E_{H}$ and $s P R E_{N}$ are the linear contributions of proton and nitrogen to the global $s P R E s_{R_{2}}$. Using this model, the intensities of the NMR signals (I) can be fitted to a quadratic equation of the type:

$$
I=a+b C+c C^{2}
$$

Anyway, using either the parameter $b$ or $c$ of the equation 2.8 resulted to be a non-robust method as their contribution vary to a great extent (data not shown. In a simpler approach, the peak heights of the paramagnetic sample $\left(\mathrm{I}_{p}\right)$ can be compared with the peak heights of the diamagnetic $\left(\mathrm{I}_{d}\right)$ sample using the ratio:

$$
s P R E_{\text {height }}=\frac{I_{p}}{I_{d}}
$$

similarly to the approach adopted by Molinari et al. (1997) and Bernini et al. (2008). As a result, the ratio of equation 2.9 is close to 1 for nuclear spins which are buried in the core of a protein and therefore far from the unpaired electron spin. The distance to the paramagnetic agent is much shorter for nuclear spins on the protein's surface and the aforementioned ratio is close to zero. 
If the sPRE evaluation is conducted using the signal intensity, it needs to be considered that the enhancements of the longitudinal relaxation rates $R_{1}$ and of the transverse relaxation rates $R_{2}$ have opposite effects on the intensity of the NMR signal. Magnetization vanishes faster from the xy plane if $\mathrm{R}_{2}$ rates are larger, thus leading to a signal reduction. On the other hand, enhanced $R_{1}$ rates speed up the recovery of the magnetization along the $\mathrm{B}_{0}$ field and produce an increase in the signal if the inter-scans delay is not long enough to guarantee a complete restoration of the Boltzmann equilibrium distribution. Although a longer inter-scans delay resulted in single experiments about 2.5 times longer in our case, the total experimental time is still much shorter than the one required for the approach used by Öster et al. (2017). In addition to this, 3D experiments can also be used to evaluate the sPREs within one week of total measurement time using the equation 2.9. Finally, a gadolinium-based paramagnetic agent, Gadolinium 1,4,7-triscarboxymethyl1,4,7,10-tetraazacyclododecane (Gd-HP-DO3A), has been used in this work as this kind of paramagnetic agents does not bind to protein samples and requires a smaller concentration compared to other paramagnetic molecules, like TEMPOL (Pintacuda and Otting, 2002).

\subsection{1 sPRE in liquid-state NMR}

Residue resolved sPRE were evaluated from ${ }^{15} \mathrm{~N}-\mathrm{HSQC}$ spectra for PASc wildtype, PASc N288D and PASc R289D using the equation 2.9. All the spectra were recorded on a Bruker $400 \mathrm{MHz}$ spectrometer equipped with a $5 \mathrm{~mm}$ triple channel room-temperature probe using an inter-scan delay of $2.5 \mathrm{~s}$. Paramagnetic spectra were acquired using $2.5 \mathrm{mM}$ of Gd-HP-DO3A.

\subsection{2 sPRE in solid-state NMR}

Residue resolved sPRE were evaluated from 3D hCANH spectra for CitApc in the bound and in the free state using equation 2.9. All the spectra were acquired on a Bruker $850 \mathrm{MHz}$ spectrometer equipped with a four channel $1.3 \mathrm{~mm}$ MAS probe using an inter-scan delay of $2.5 \mathrm{~s}$. Paramagnetic samples with $5.0 \mathrm{mM}$ of GdHP-DO3A were obtained soaking a $1.3 \mathrm{~mm}$ rotor previously packed with protein sample in $0.5 \mathrm{ml}$ of $20 \mathrm{mM}$ sodium phosphate $(\mathrm{pH} 6.5)$ and $5 \mathrm{mM}$ Gd-HP-DO3A buffer for an overnight. 


\subsection{Structure determination}

Calculations of the PASp R93A structures in the bound and in the free state were performed with the ARIA2 program (Rieping et al., 2006). The distance constraints were derived from the integral intensity of NOE cross-peaks in 3D ${ }^{15} \mathrm{~N}$ and ${ }^{13} \mathrm{C}$ resolved $\left[{ }^{1} \mathrm{H}^{1} \mathrm{H}\right]$ NOESY spectra with 100 and $80 \mathrm{~ms}$ mixing time, respectively. Chemical shift values were used to define torsion angle restraints with TALOS+ (Shen et al., 2009). The summary of the constraints used in the calculations is presented in table 2.5. The structures were validated with the PSVS suite (Bhattacharya et al., 2007).

\begin{tabular}{ccc}
\hline & PASp R93A (free) & PASp R93A (bound) \\
\hline Distance restraints & 188 & 233 \\
Dihedral restraints & & \\
phi & 122 & 127 \\
psi & 122 & 127 \\
\hline
\end{tabular}

Table 2.5: Restraints used for the structure determination of PASp R93A in the citrate-free and in the citrate-bound state 


\section{Results}

\subsection{The periplasmic PAS domain}

Crystallization trials for the isolated Geobacillus Thermodenitrificans CitA PASp domain were not successful at first. Therefore, the NMR-based structure of the PASp $_{33-161}$ R93A mutant were calculated for both citrate free (figure 3.1 left in red) and citrate bound state (figure 3.1 right in blue) using ARIA2 (Rieping et al., 2007). The structure statics for the ensembles of the best 20 structures after water refinement are reported in table 3.1 .

\begin{tabular}{lcc}
\hline & PASp R93A (free) & PASp R93A (bound) \\
\hline Structure statistics & & \\
\hline Distance constraints violation, $[\AA]$ & $0.035 \pm 0.011$ & $0.102 \pm 0.049$ \\
Dihedral constraints violation, $\left[{ }^{\circ}\right]$ & $0.61 \pm 0.28$ & $0.62 \pm 0.18$ \\
Max distances violation, $[\AA]$ & $1.592 \pm 1.291$ & $2.651 \pm 1.725$ \\
Max dihedrals violation, $\left[^{\circ}\right]$ & $15.24 \pm 3.04$ & $26.54 \pm 7.42$ \\
\hline Backbone rms deviations & & \\
Entire protein & $2.953 \pm 0.498$ & $2.269 \pm 1.019$ \\
Ordered regions & $2.465 \pm 0.523$ & $2.158 \pm 1.099$ \\
\hline
\end{tabular}

Table 3.1: Structure statics of the NMR-based structure of the $\mathrm{PASp}_{33-161}$ R93A with and without citrate.

A mean distance constraints violation of $0.035 \pm 0.11 \AA$ and a mean dihedral constraints violation of $0.61^{\circ} \pm 0.28^{\circ}$ is found for the structure of the PASp R93A in the free state. The RMSD calculation for the ensemble of the same sample produced a value of $2.953 \pm 0.498 \AA$ for the entire protein and in $2.465 \pm 0.523$ $\AA$ taking into account only the ordered region.

For the citrate bound state, a value of $0.102 \pm 0.049 \AA$ and of $0.62^{\circ} \pm 0.18^{\circ}$ is found for the mean distance constraints violation and the mean dihedral constraints violation, respectively. The backbone RMSD resulted in $2.269 \pm 1.019 \AA$ and $2.158 \pm 1.099 \AA$ for the entire protein and for the ordered region, respectively. From the analysis of the dihedral angles, only 8 outliers are found for the PASp R93A in the free state, with $92.1 \%(585 / 635)$ of all the dihedral angles in the favoured region and $98.7 \%(627 / 635)$ in the allowed region.

For the bound state of the PASp R93A, 11 outliers are present, with $92.4 \%$ $(587 / 635)$ of all the dihedral angles in the favoured region and $98.3 \%(624 / 635)$ 
in the allowed region.

Recently, diffracting crystals of citrate-bound and of citrate-free $G$. thermodenitrificans CitA PASp R93A were obtained. Structures were solved by Dr. Stefan Becker (Department of NMR-based Structural Biology, Max-Planck-Institute for Biophysical Chemistry, Göttingen, GER). In figure 3.1, the overlap of citrate-free (left) and citrate-bound (right) PASp crystal structures (in grey and black) with the NMR model ( in red and blue) are shown. A dimeric interface was found in the crystals only in absence of the citrate. For the bound state, the dimeric structure was generated from the structural alignment of two monomer unit of the holo-protein with the dimer of the apo-protein. NMR models and crystal structures agree very well, with a backbone RMSD of $1.864 \AA$ for the bound state and of $2.931 \AA$ for the free state, when the entire protein is used for the calculation. If only the ordered regions are analysed, the RMSD calculation produce a value of $1.384 \AA$ and $1.368 \AA$ for the bound and the free state, respectively.
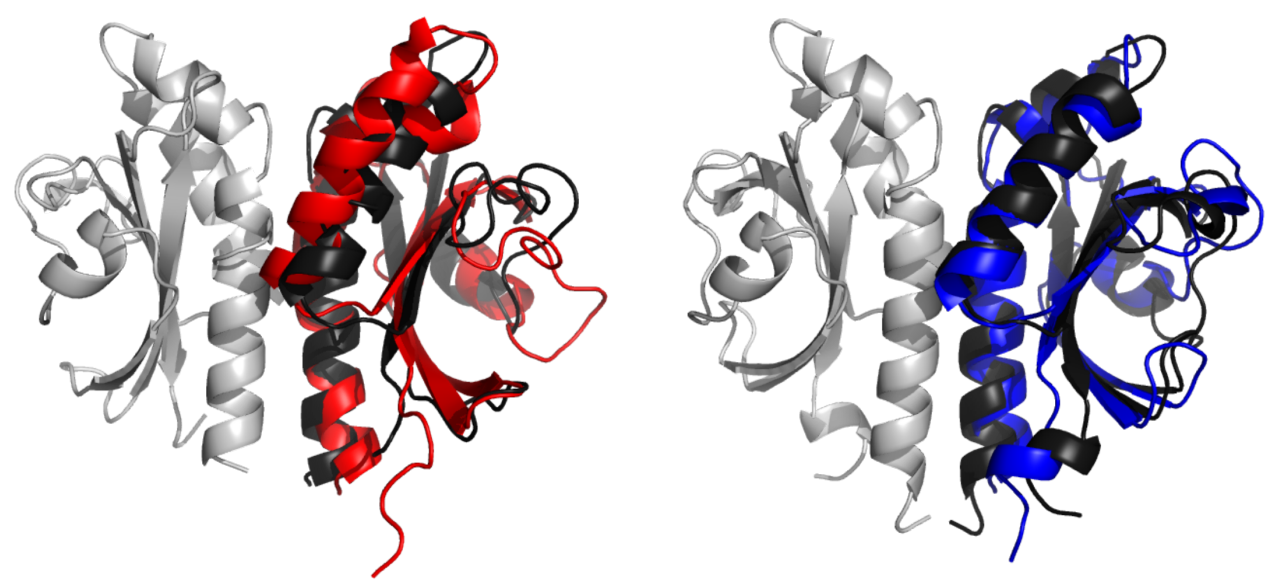

Figure 3.1: Crystal structures and NMR models of the CitA PASp. The NMR models are coloured in red for the citrate-free state and in blue for the citrate-bound. Crystal structures are represented in grey and black and overlapped with the corresponding NMR models.

\subsection{The cytosolic PAS domain}

The cytosolic PAS domain is responsible to translate the output signal of the TM domain in the input signal of the kinase core. It does not bind any ligand and 
does have any interaction partner. Therefore, it is not possible to say whether the wild-type sample of the isolated construct represent in this case the ON or the OFF state. In DcuS, another HK of the CitA family, PASc mutants were tested for effects on the activity of the full-length construct (Monzel et al., 2013). The PASc mutants associated with alterations in DcuS activity can be divided in:

- OFF-mutants, which trap DcuS in the inactive state

- ON-mutants, which trigger signalling even in absence of dicarboxylates

In addition, OFF-mutants always conserve the functional dimeric state and binding capability to DctA, while the ON-mutants can be divided further. The ON I-subtype destroys the HK dimer; thus, this class does not correspond to biologically relevant states. The ON II-subtype retains the dimeric state; ON IIB-mutants also maintain the binding capability to DctA, while this interaction is lacking in ON IIA-mutants.

\begin{tabular}{|c|c|c|c|c|c|c|}
\hline \multirow{4}{*}{$\begin{array}{l}G \text {. } \\
E \text {. }\end{array}$} & \multirow{4}{*}{$\begin{array}{l}\text { thermodenitrificans } \\
\text { coli Dcus }\end{array}$} & 200 & 210 & 220 & 230 & 240 \\
\hline & & peEIGLLYQE & KQAILEAIRE & GIVAINQEGT & ITMVNQTALK & LLgydnernv \\
\hline & & PYEISTLFEQ & RQAMLQSIKE & GVVAVDDRGE & VTLINDAAQE & LLnyrksqdd \\
\hline & & 214 & 224 & 234 & 244 & 254 \\
\hline & & 250 & 260 & 270 & 278 & 288 \\
\hline$G$. & thermodenitrificans CitA & lgtpilqlip & hsrlpevirt & gqaey--DDE & MVLGGETVIA & NRIPIKnkqG \\
\hline$E$. & coli Dcus & eklstlshsw & sqvvdvsevl & rdgtprrDEE & ITIKDRLLLI & NTVPVRsn-G \\
\hline & & 264 & 274 & 284 & 294 & 304 \\
\hline & & 298 & 308 & & & \\
\hline & thermodenitrificans CitA & RVIGAVSTER & NK & & & \\
\hline & coli Dcus & VIIGAISTER & DK & & & \\
\hline & & 313 & 323 & & & \\
\hline
\end{tabular}

Figure 3.2: Alignment of Geobacillus thermodenitrificans CitA PASc and Escherichia coli DcuS PASc. Functional mutants in PASc of E. coli DcuS were transferred to $G$. thermodenitrificans CitA based on a sequence alignment. Transferred on mutants are highlighted in green, OFF-mutants are shown in red. For DcuS N304D, R289 neighbouring the aligned N288 in CitA was selected as a second potential candidate for mutation. Capital letters indicate high sequence homology.

Mutants of $G$. thermodenitrificans CitA PASc were generated based on the analysis conducted in vivo on the PASc domain of homologous E. coli DcuS by Monzel et al. (2013). Conserved residues in G. thermodenitrificans CitA PASc that were mutated in E. coli DcuS PASc were identified based on a sequence alignment of the two proteins generated with Dialign (Morgenstern, 2004) (see figure 3.2). While the selection was not restrained for the OFF-mutants, the ON-mutants were chosen only from the ON II-subtype as these retain the functional dimeric state in 
DcuS. In the case of N288 in G. thermodenitrificans CitA PASc, the neighbouring R289, being a polar residue as well, was selected as a second possible mutant to rule out errors in the sequence alignment. Like PASc wild-type, all the proposed point mutants are dimeric in solution based on the SEC profiles (see figure 3.3) and the dimeric state resulted to be stable down to a concentration of $15 \mu \mathrm{M}$ for the CitA PASc N288D . In vivo testing of the kinase activity of the PASc mutants

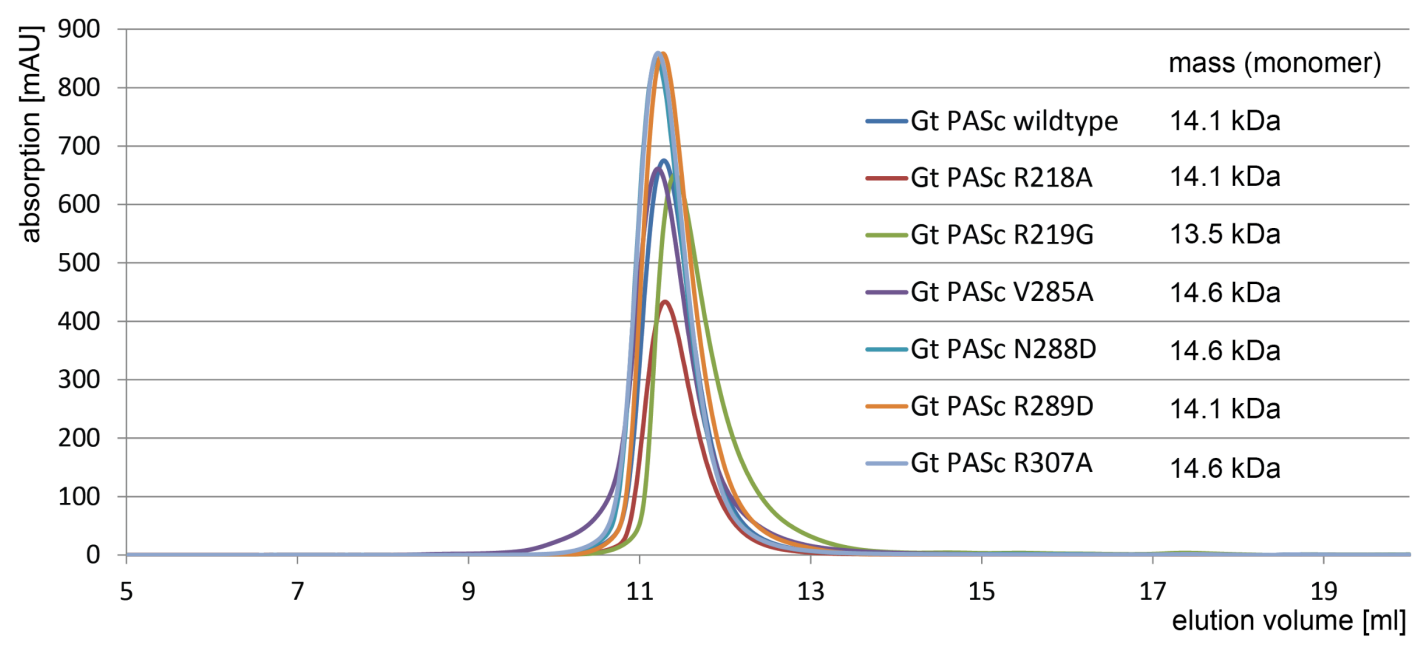

Figure 3.3: Size exclusion chromatography profile of the proposed $G$. thermodenitrificans CitA PASc mutants. The molecular weight can be calculated from a calibration and are reported for PASc mutant monomers. The SEC profile of wild-type PASc is shown as a reference.

was performed in the lab of Prof. Unden, but, in contrast to DcuS, no difference to wild-type $G$. thermodenitrificans CitA was observed in the kinase activity for any of the mutants. (see figure 3.4)

Crystallization trials were carried out for all the $G$. thermodenitrificans CitA PASc point mutants. Crystal structures were solved for the proposed OFF-mutants CitA PASc R218A and V285A and for the proposed ON-mutants N288D, R289D and R307A by Dr. Stefan Becker (Department of NMR-based Structural Biology, MaxPlanck-Institute for Biophysical Chemistry, Göttingen, GER). All structures are found in a dimeric state and they all adopt a similar PAS fold for the monomer unit, with one N-terminal $\alpha$-helix, a $\beta$-sheet made of 5 anti-parallel strand in the core and an outer loop forming four short $\alpha$-helices. Excluding the N-terminal $\alpha$ helix, the fold of the individual domain is very well maintained with a maximum RMSD of $0.42 \AA$. In contrast, the N-terminal $\alpha$-helix is found in three different orientations, which strongly affect the dimer arrangement (see table 3.2 and figure $3.5)$. 


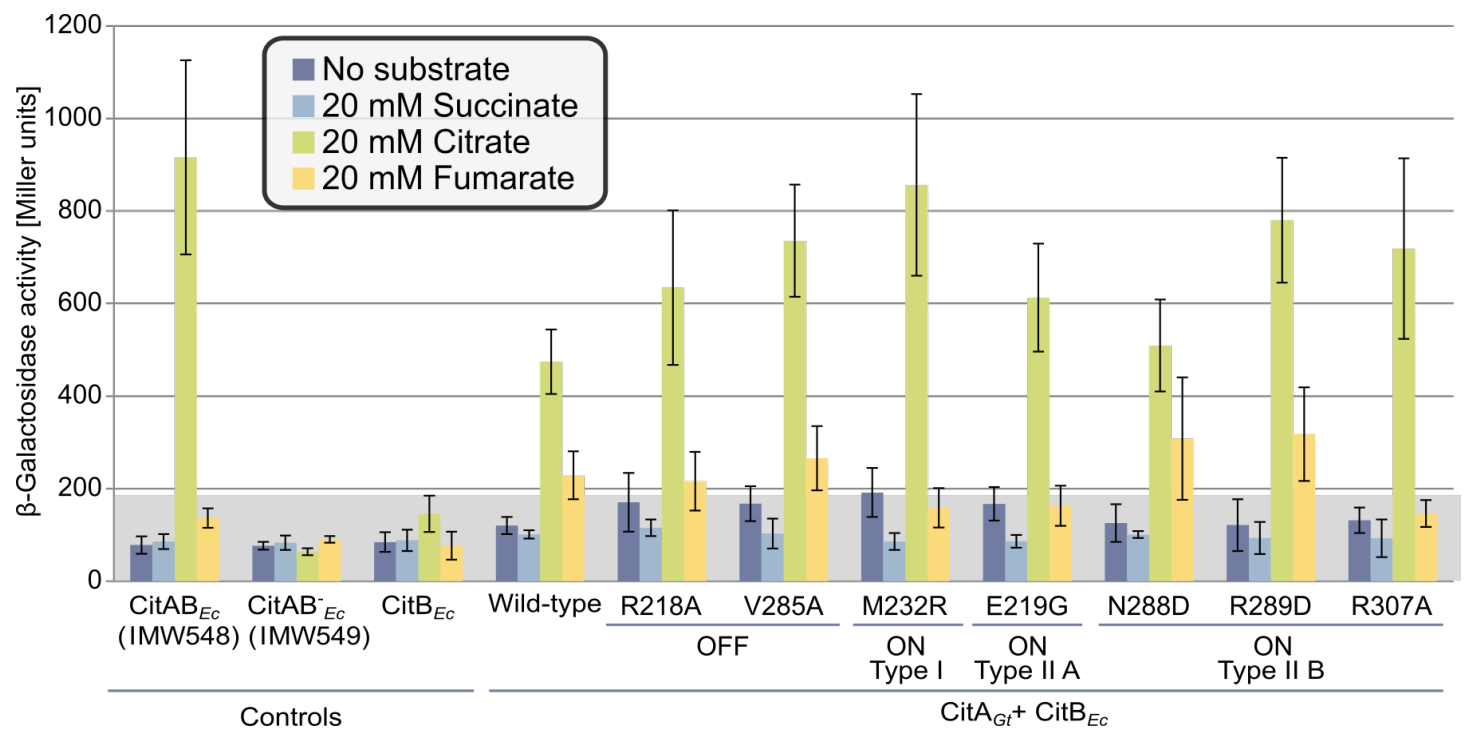

Figure 3.4: In vivo testing of the proposed $G$. thermodenitrificans CitA PASc functional mutants performed in Prof. Unden lab. In dark blue is shown the value in absence of the substrate; in light blue, green and yellow are shown the activities in presence of succinate, fumarate and citrate, respectively.

\begin{tabular}{|c|c|c|c|c|}
\hline DcuS mutation & In vivo activity & CitA mutation & In vivo activity & Dimer arrangement \\
\hline wild-type & like wt & wild-type & \multirow{7}{*}{ like wt } & parallel \\
\hline K232A & \multirow{2}{*}{$\mathrm{OFF}$} & $\mathrm{R} 218 \mathrm{~A}$ & & anti-parallel \\
\hline L301A & & V285A & & parallel \\
\hline E233G & ON Type IIA & E219G & & no crystals \\
\hline N304D & \multirow{3}{*}{ ON Type IIB } & N288D & & anti-parallel \\
\hline N304D & & R289D & & open \\
\hline R322A & & R307A & & parallel \\
\hline
\end{tabular}

Table 3.2: Summary of in vivo testing on E. coli DcuS and G. thermodenitrificans CitA functional mutations. The last column summarizes the possible dimer arrangements of the G. thermodenitrificans CitA PASc crystal structures.

For the CitA PASc wild-type and the R307A and V285A mutants, a classical PAS dimer is found, where the N-terminal helices exchange between dimer units and they run parallel to each other (see figure $3.5 \mathrm{~A}$ ). This has been named the "parallel" dimer (PD). A different dimer orientation is found for the CitA PASc R289D mutant, where the two N-terminal helices lose contact between each other and interact only with the central $\beta$-sheet scaffold in trans to create an "open" dimer (OD) (see figure $3.5 \mathrm{~B}$ ). A third dimer arrangement, named "anti-parallel" dimer (AD), is found for the CitA PASc N288D and R218A mutants. In this case, the helices run anti-parallel to each other and the contact between the N-termini and the central $\beta$-scaffold of the opposite dimer unit is lost (see figure $3.5 \mathrm{C}$ ).

To prove that the anti-parallel and open dimer arrangements are not a crystal 

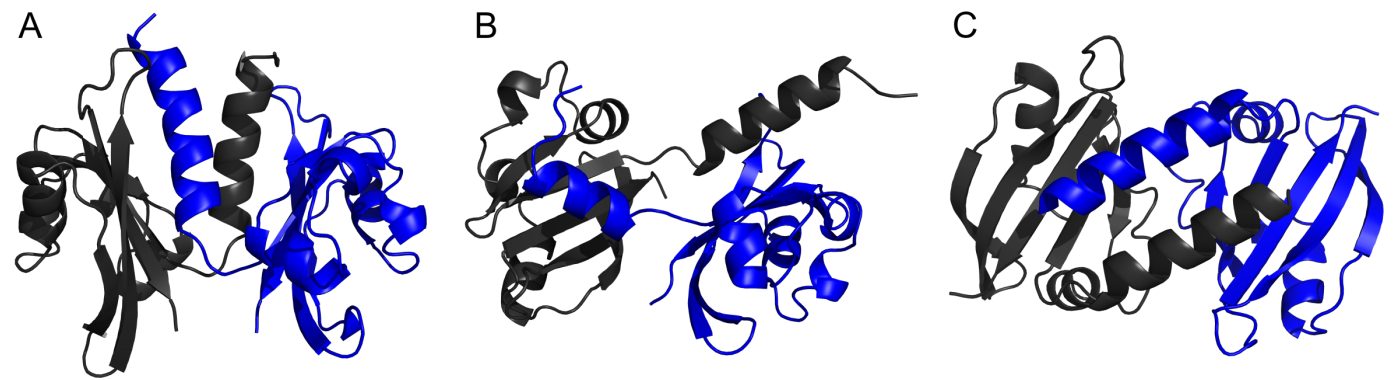

Figure 3.5: Three dimer arrangements for the CitA PASc wild-type and mutants (monomers coloured in black and blue). (A) The crystal structure of CitA PASc wild-type shows a classical PAS dimer arrangement, called "parallel" dimer, found also for the CitA PASc R307A and V285A mutants. (B) The structure of CitA PASc R289D reveals a different dimer orientation, called "open" dimer. (C) The crystal structure of CitA PASc N288D displays a third option, called "anti-parallel" dimer, which was also found for the CitA PASc R218A.

packing artefacts, G. thermodenitrificans CitA PASc N288D and R289D mutants were assigned using liquid-state NMR experiments (see table 2.2). In total $74 \%$ of proton, $80 \%$ of carbon and $67 \%$ of nitrogen resonances were assigned for CitA PASc R289D and $70 \%$ of proton, $80 \%$ of carbon and $67 \%$ of nitrogen resonances were assigned for CitA PASc N288D. Of all the backbone resonances, 93\% were assigned for CitA PASc R289D and 95\% for CitA PASc N288D. The $\mathrm{C}_{\alpha}$ chemical shift differences between CitA PASc wild-type and the N288D and R289D mutants are reported in figures $3.6 \mathrm{~A}$ and 3.6B. In both cases, the largest differences are found in the $\mathrm{N}$ terminal region that forms the the $\alpha$-helix 1 (highlighted in blue) and at the residues that are either close in the sequence or in space to the mutation site (highlighted in green).

Amide hydrogen exchange patterns were also analysed for the three samples. After dialysing each sample for two days against deuterated buffer, a ${ }^{15} \mathrm{~N}-\mathrm{HSQC}$ spectrum of each sample was measured. In figure 3.7 are reported the ratios between the peak intensities of the spectra acquired using $20 \mathrm{mM}$ sodium phosphate deuterated buffer at $\mathrm{pH} 6.5$ with those of the reference spectra, acquired using 20 $\mathrm{mM}$ sodium phosphate buffer also at $\mathrm{pH} 6.5$.

Of the 110 residues of the CitA PASc domain, 19 are still visible in the wild-type sample, 35 in the N288D mutant and 31 in the R289D mutant. The amide protons in the central $\beta$-sheet are in general well protected from exchange with deuterium, especially the first strand from residue 220 to residue 225 . The main differences 


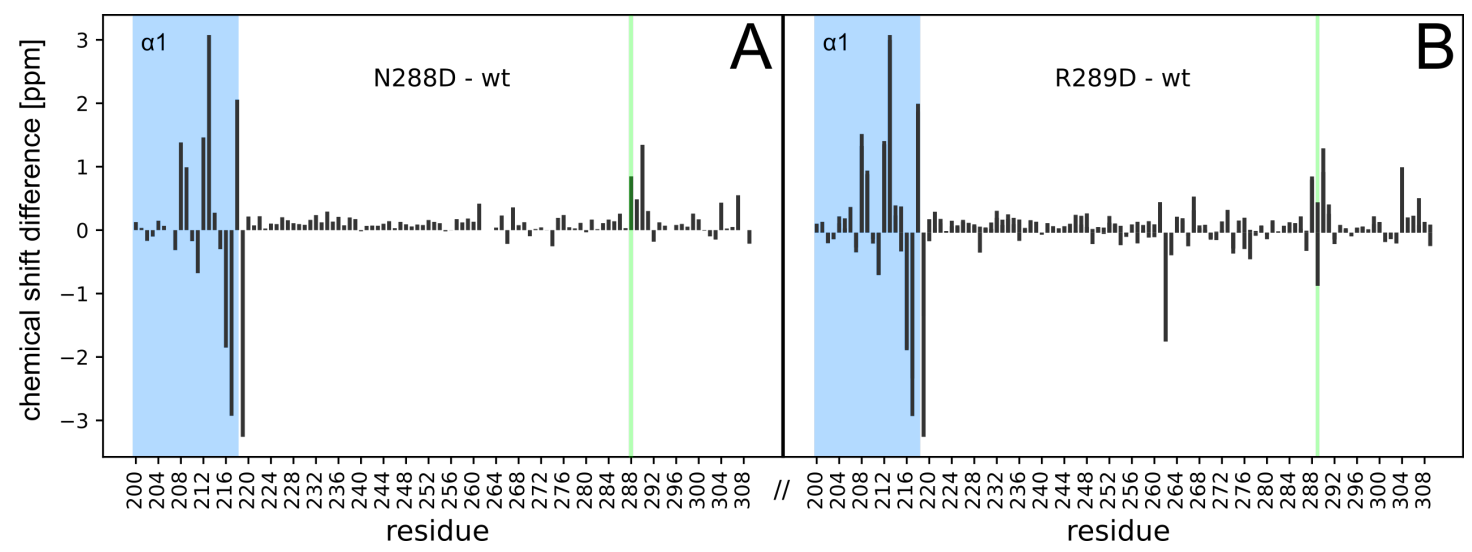

Figure 3.6: $\mathrm{C}_{\alpha}$ chemical shift differences of A: CitA PASc N288D with wild-type and B: CitA PASc R289D with wild-type. In blue are the residues that form the N-terminal $\alpha$-helix, in green the mutation site.

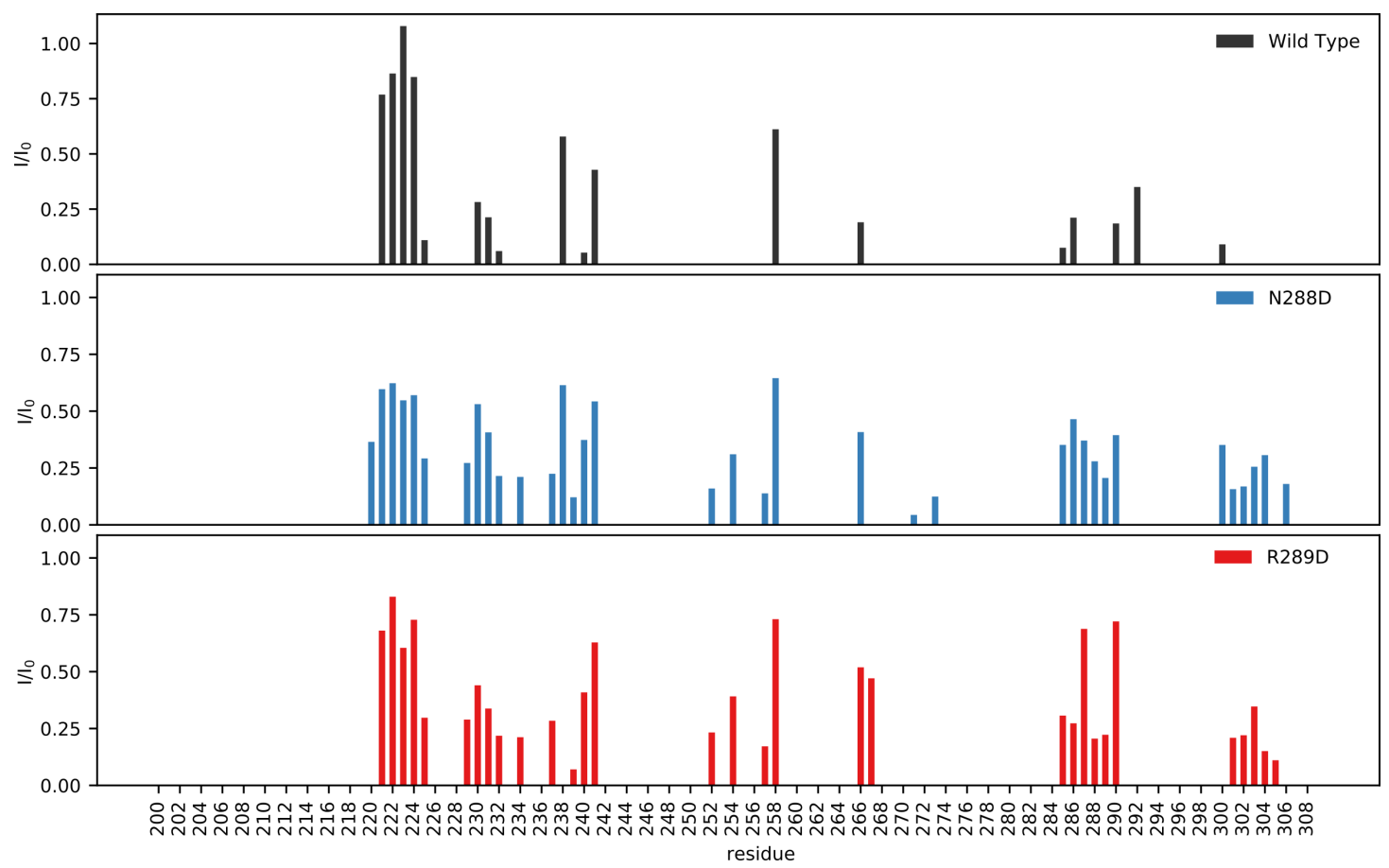

Figure 3.7: Amide proton exchange patterns in the PASc wild type (top, in black), N288D (middle, in blue) and R289D mutants (bottom, in red). The ratio between the intensity after the exchange $\left(I_{0}\right)$ and the intensity before the exchange (I) is plotted in function of the residue number.

between wild-type PASc and the two mutants are located at the last $\beta$-strand (residues 300-309), where the amide protons are exchanging with deuterium in the PASc wild-type much faster than in the two mutants. 
Amide exchange patterns result from the combination of both structural and dynamic features of a protein, therefore they do not reflect the dimer interface differences faithfully. Solvent PRE has proved to be very useful for the identification of the solvent-exposed regions of a protein and for the detection of intermolecular contact sites (Pintacuda and Otting, 2002) and it does not depend on the stability of secondary structure elements. To measure sPRE effects on CitA PASc, samples of the wild-type and of the N288D and R289D mutants were doped with $2.5 \mathrm{mM}$ of Gd-HP-DO3A and the intensity ratio between the paramagnetic and the diamagnetic ${ }^{15} \mathrm{~N}-\mathrm{HSQC}$ spectra was used to monitor the effect of the dopant agent. The profile of the intensity ratio shows little differences between the samples, with the only significant discrepancies located between residue 216 and 219 (see figure 3.8 ). In this part, a lower ratio is obtained only for the PASc N288D mutant, suggesting that these residues are closer to the protein surface in comparison with the other two samples.

The experimental values were compared to the predicted sPRE obtained by applying the algorithm provided by Öster et al. (see figure 3.8 bottom) on the crystal structures of figure 3.5. The output of the algorithm is a number indicating the linear enhancement of the relaxation rate of a spin as a function of the dopant concentration. Therefore, high values are associated with atoms close to the protein surface and low values with atoms buried in the core, as opposed to the aforementioned intensity ratio used for the experimental sPRE analysis (see section 2.4). The deviations for the open dimer conformation are significant in the N-terminal helix, but they are not reliable as the first 5 residues are not well defined in the corresponding crystal structure. Other differences can be localized between residues 216 and 218, where the AD structure shows higher sPREs than the OD and PD conformation in agreement with our experimental values, as highlighted by the green dashed box in figure 3.8. Thus, sPRE measurement confirmed the existence of the anti-parallel dimer arrangement also in solution, but was unable to discern between the parallel and open dimer conformations because their protein surfaces exhibit too little differences if the unstructured residues at the N-terminus in the crystal structures are excluded.

The different dimer arrangements were further characterized using Cryogenic Optical Localization in 3D (COLD) (Weisenburger et al., 2017) as well as NOESY experiments. The COLD measurements were conducted in the group of Prof. Sandoghdar (Max Planck Institute for the Science of Light, Erlangen, GER). 


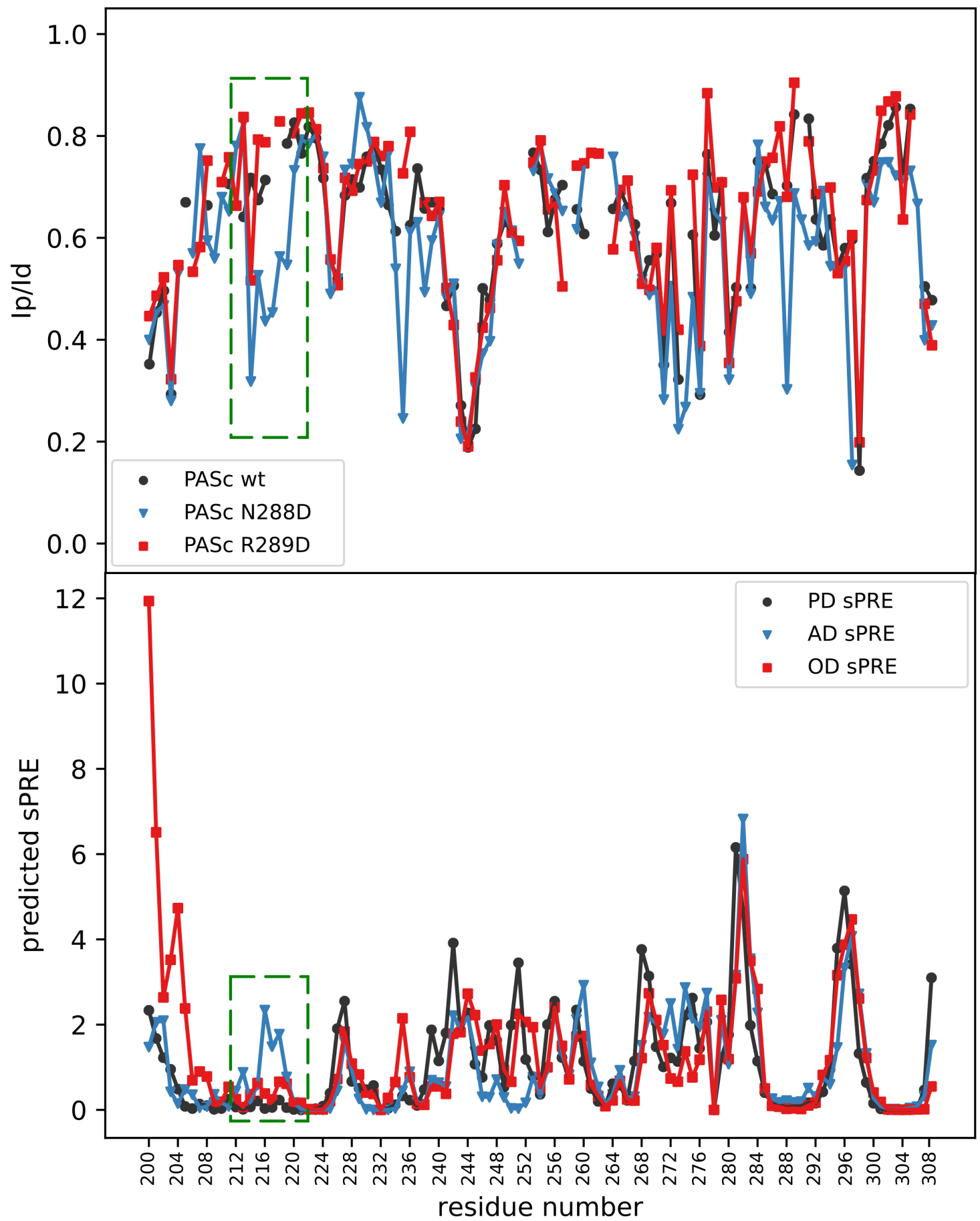

Figure 3.8: Top: profile of intensity ratios between the paramagnetic ( 2.5 $\mathrm{mM}$ of Gd-HP-DO3A) and diamagnetic (0 mM of Gd-HP-DO3A)sample for the PASc wild-type (black circles), PASc N288D (blue triangles) and PASc R289D (red squares). Bottom: predicted sPRE values for the parallel dimer (black cicles), anti-parallel dimer (blue triangles) and open dimer (red squares). The green dashed box highlights the similarities between the predicted and the experimental values.

The inter-unit distance at the C-terminus was determined for the PASc wild-type (Weisenburger et al., 2017), the N288D and R289D mutants, with a corresponding 
experimental value of $9 \AA, 40 \AA$ And $20 \AA$. These distances are in excellent agreement with the one expected from the crystal structure and confirm that CitA PASc wild-type, N288D and R289D mutants adopt respectively the "parallel", the "anti-parallel" and the "open" dimer arrangement (see table 3.3).

To analyse the dimer interface of $G$. thermodenitrificans CitA PASc via NMR,

\begin{tabular}{|c|c|c|}
\hline Dimer arrangement & Crystal structure & COLD measurements \\
\hline Parallel & $9.4 \AA$ & $9 \AA$ \\
\hline Anti-parallel & $48.3 \AA$ & $41 \AA$ \\
\hline Open dimer & $22.7 \AA$ & $21 \AA$ \\
\hline
\end{tabular}

Table 3.3: Comparison between the $\mathrm{N} 308 \mathrm{C} \alpha$-C $\alpha$ inter-unit distances calculated on the crystal structures and measured with COLD.

a $3 \mathrm{D}{ }^{13} \mathrm{C} /{ }^{15} \mathrm{~N}$ double-edited-NOESY (Kay et al., 1990) was measured on a sample where ${ }^{13} \mathrm{C}$ labelled CitA PASc was mixed with ${ }^{15} \mathrm{~N}$ labelled CitA PASc in a 1:1 ratio. With this approach, the NOE cross peaks are generated only from through-space contacts at the dimer interface. Based on the chemical shift matching assignment, it was impossible to correlate unequivocally one sample to only one dimer arrangement. Indeed, for each sample at least one cross-peak was found that unambiguously fits the distances estimated from the "parallel" dimer, one that fits the distances from the "anti-parallel" dimer and one that fits the distances from the "open" dimer (see table A.1).

Nitrogen Chemical Exchange Saturation Transfer $\left({ }^{15} \mathrm{~N}\right.$ CEST) experiments were
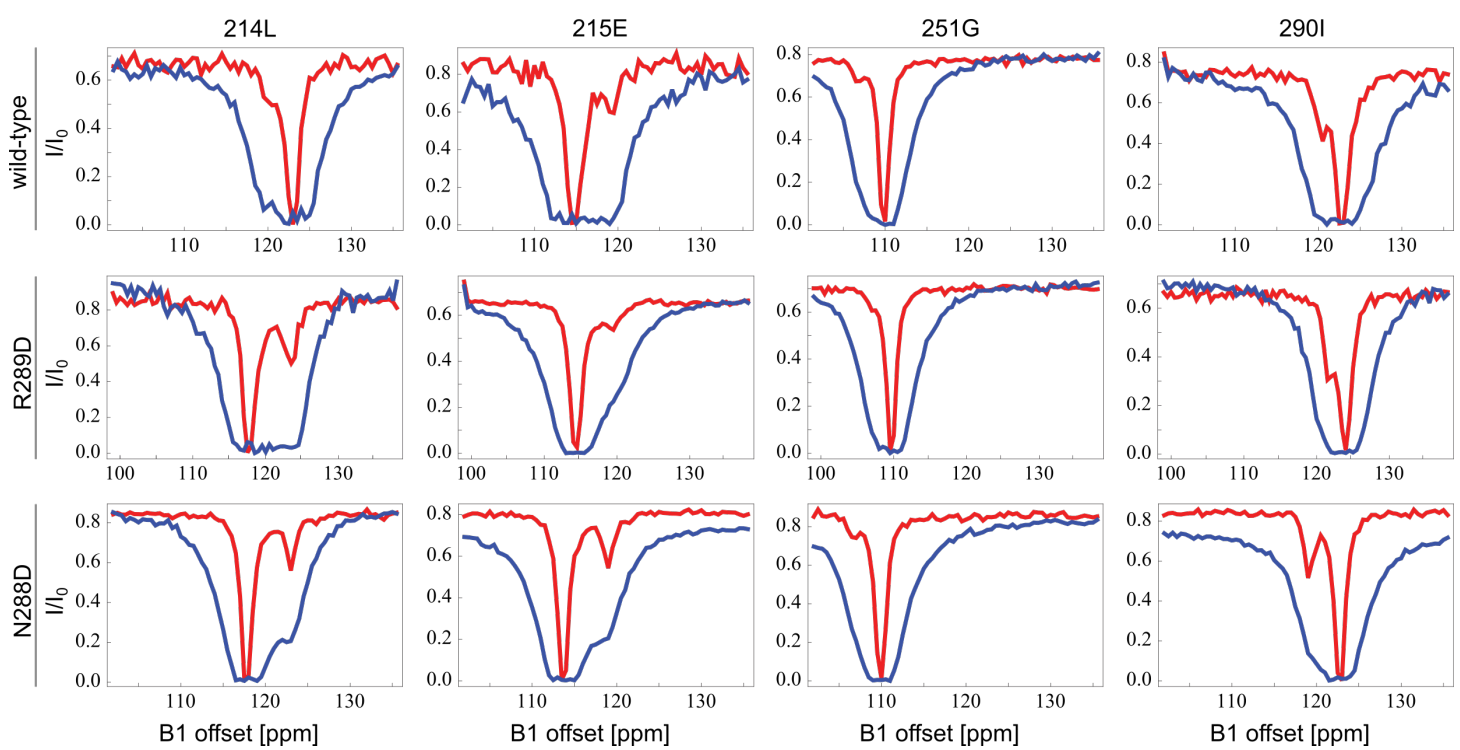

Figure 3.9: Representative ${ }^{15} \mathrm{~N}$ CEST profile for the CitA PASc wild-type (top), R289D mutant (middle) and N288D mutant (bottom). The red and the blue profile represent ${ }^{15} \mathrm{~N} \mathrm{~B}_{1}$ field of $20 \mathrm{~Hz}$ and $80 \mathrm{~Hz}$, respectively. 

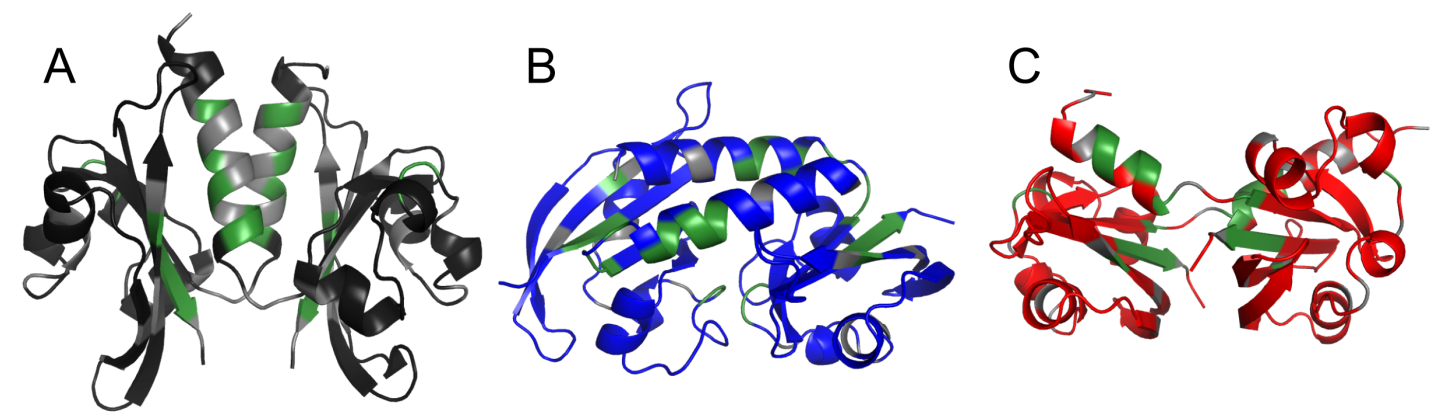

Figure 3.10: The residues showing exchange for the CitA PASc wild-type (A and black), the N288D mutant (B and blue) and the R289D mutant (C and red) samples are plotted on the respective crystal structures and coloured in green. For the residues coloured in grey no ${ }^{15} \mathrm{~N}$ CEST profile is available. The amino-acids showing exchange cluster on the elements forming the dimer interface, namely the N-cap $\alpha$-helix, the fourth and the fifth $\beta$-strand and the major loop. The latter is involved in inter-subunit contacts only in the crystal structure of PASc N288D, but shows exchange in all the samples.

also measured on the $G$. thermodenitrificans CitA PASc wild-type and on the N288D and R289D mutants. The representative CEST profile for the PASc wildtype, the PASc N288D and the PASc R289D are reported in figure 3.9. The presence of the minor dip indicates the existence of an alternative structure in slow exchange with the main conformation. Out of 93 analysed profiles, 11 residues displayed a second dip in the two PASc mutants and 12 for the PASc wild-type. Moreover, all of the amino-acids showing exchange properties cluster at the interface of the three possible dimer arrangements (see figure 3.10)

\subsection{The liposome embedded construct CitApc}

\subsection{1 ${ }^{13} \mathrm{C}$ detected solid-state NMR}

G. thermodenitrificans CitApc wild-type and R93A mutant samples embedded both in asolectin and in pure 1,2-dimyristoyl-sn-glycero-3-phosphocholine (DMPC) were already measured and assigned in this lab using ${ }^{13} \mathrm{C}$ detected solid-state NMR experiments (Schomburg, 2014). The assignment was satisfying for the periplasmic domain, where $86 \%$ and $81 \%$ of the residues could be assigned in the citrate-bound and -free sample respectively, but it left open many questions about the ON-OFF transitions, especially about the cytosolic PAS domain, where only $59 \%$ of the 
apo-CitApc and $45 \%$ of the holo-CitApc were assigned, and about the two transmembrane helices, which were not visible.

In the attempt to gain structural information about the residues which are embedded in the membrane or are found at the dimer interface, G. thermodenitrificans CitA PASpc samples were soaked in deuterated buffer. With this approach, only the residues protected from exchange with the solvent, ideally the core of the protein, the transmembrane region and the dimer interface, are still visible in the spectra. The overall NMR signal of the $\mathrm{D}_{2} \mathrm{O}$ washed samples was reduced by a factor of three, but spectra were less crowded (see figure 3.11). Based on the avail-
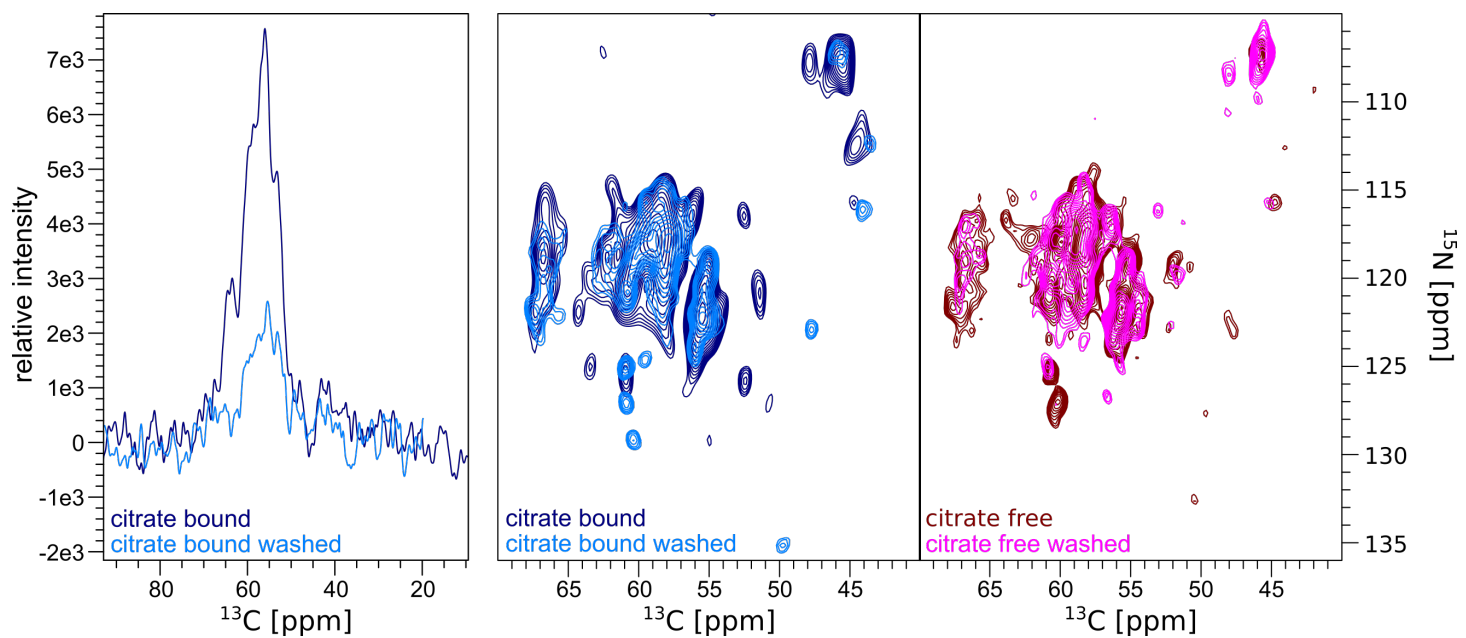

Figure 3.11: Effect of washing with deuterated buffer. On the left the 1D NCA of the citrate bound PASpc, in the middle the 2D NCA plane for the PASpc citrate bound, on the right the 2D NCA plane for the citrate free sample.

able chemical shift values, it was possible to assign a total of 25 cross-peaks in the NCA 2D plane for the free state and 24 for the bound state. The distribution of the assigned residues on the sequence is very different between the holo and the apo state of the CitApc. In the periplasmic domain, 21 residues retained visibility in the presence of the ligand, while only 17 were visible in the free state. This trend is inverted for the cytosolic domain, where five residues retained the signal for the free state and no signal was observed for the bound state. Finally, using the chemical shifts obtained for the CitApc reconstituted in asolectin, it was possible to assign 3 residues of the TM2 (see figure 4.9). 


\subsection{2 $\quad{ }^{1} \mathrm{H}$ detected approach development}

Proton detected solid-state NMR techniques developed quickly in the last years and have many advantages over the ${ }^{13} \mathrm{C}$ detected solid-state NMR. Samples of $G$. thermodenitrificans CitA PASpc with different conditions of buffer, $\mathrm{pH}$ and lipid composition (see table 3.4) have been tested to optimize the spectral quality.

\begin{tabular}{|c||c||c|}
\hline Lipids & Buffer & $\mathrm{pH}$ \\
\hline DMPC & 20 mM sodium phosphate & 6.0 \\
DMPC/DMPA (9:1) & 20 mM TRIS & 6.5 \\
DPPC/DPPA (9:1) & & 7.0 \\
& & 7.4 \\
\hline
\end{tabular}

Table 3.4: Condition used for lipid composition, buffer and $\mathrm{pH}$ screened for the sample optimization.

First, buffer conditions already adopted for the purification and crystallization of the isolated PAS domains were also used for the reconstitution in liposomes of CitApc. Resolution of the NCA plane improved visibly changing buffer conditions from $20 \mathrm{mM}$ TRIS at $\mathrm{pH} 7.4$ to sodium phosphate buffer at $\mathrm{pH} 6.5$ (see figure 3.12 A and B). Second, a 9:1 DMPC/DMPA (1,2-dimyristoyl-sn-glycero-3-phosphate) ratio was used for the liposomes to simulate the negative charge of the bacterial membrane and to avoid the fusion of the liposomes. This last step further improved spectral quality (see figure $3.12 \mathrm{~B}$ and $\mathrm{C}$ ).

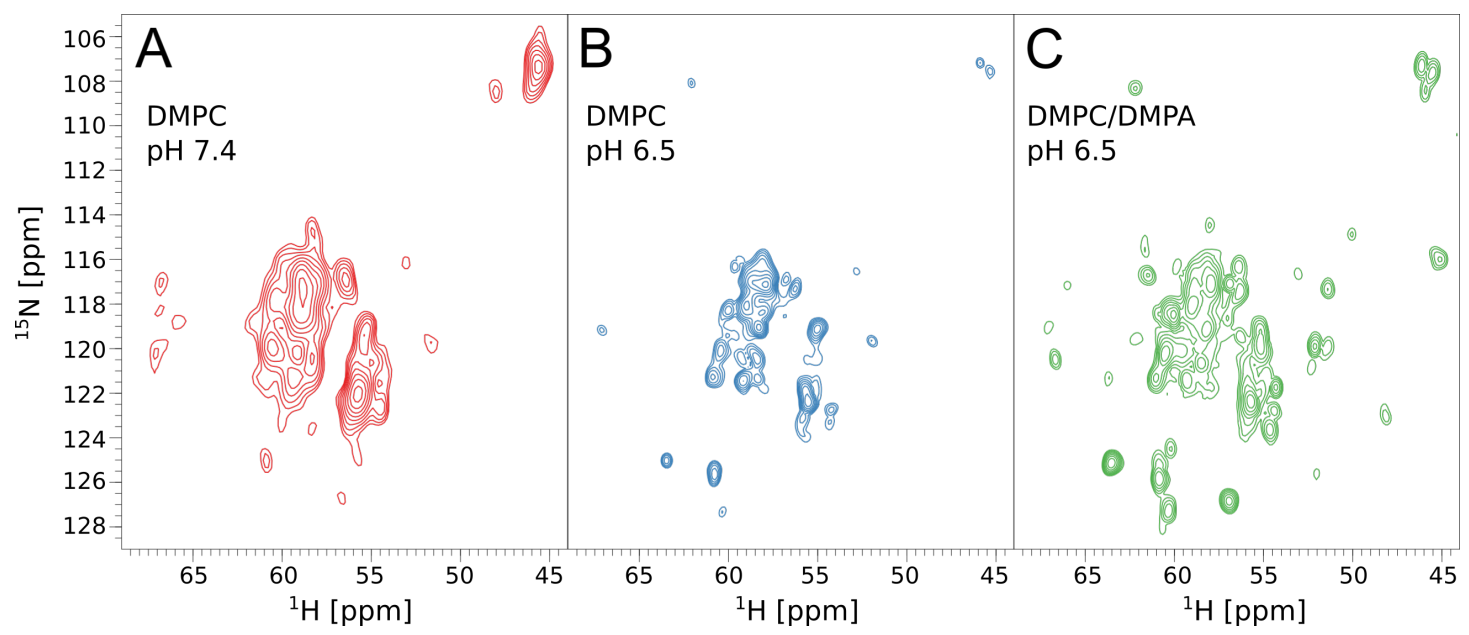

Figure 3.12: NCA plane for the CitA PASpc R93A citrate free in pure DMPC using in $20 \mathrm{mM}$ TRIS buffer at $\mathrm{pH} 7.4$ (A) and the hCANH projection of the CitA PASpc C12AR93A citrate free in pure DMPC using $20 \mathrm{mM}$ sodium phosphate buffer at $\mathrm{pH} 6.5$ (B) and in DMPC/DMPA 9:1 mixture using $20 \mathrm{mM}$ sodium phosphate buffer at $\mathrm{pH} 6.5(\mathrm{C})$. 
Other samples were tested to determine the ideal $\mathrm{pH}$ value and liposomes' thickness. The major fatty acids present in Geobacillus thermodenitrificans are saturated with 15, 16 or 17 carbon atoms (Feng et al., 2007), while DMPC fatty acid chain comprises of only 14 carbon atoms; thus, lipids with a longer chain were sought and a 9:1 mixture of 1,2-dipalmitoyl-sn-glycero-3-phosphocholine (DPPC) and 1,2-dipalmitoyl-sn-glycero-3-phosphate (DPPA) was selected for testing. Based on ${ }^{15} \mathrm{~N}$ line-width analysis, the 9:1 DMPC/DMPA ratio, i.e. the shorter lipids, still resulted in better spectral quality (see table 3.5). The 9:1 DMPC/DMPA mixture was also tested at pH 6.0 and at $\mathrm{pH}$ 7.0. Sample quality is comparable at $\mathrm{pH} 6.0$ and $\mathrm{pH}$ 6.5, while it decreases for $\mathrm{pH} 7.0$ (see table 3.5). However, $\mathrm{pH} 6.5$ was preferred as the same value is used for liquid-state NMR samples.

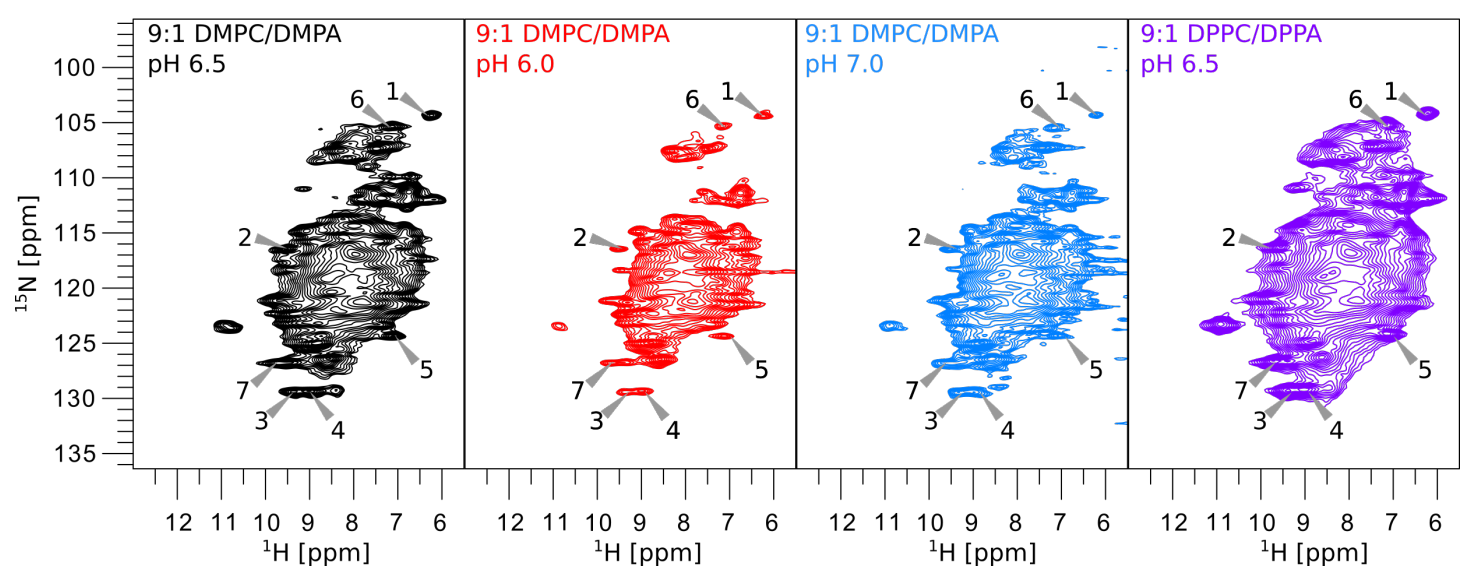

Figure 3.13: The $2 \mathrm{D}{ }^{1} \mathrm{H}_{-}{ }^{15} \mathrm{~N}$ correlation spectra of citrate-bound CitApc C12A used for sample optimization.

\begin{tabular}{|r|c|c|c|c|c|}
\hline \multicolumn{5}{|c|}{${ }^{15}$ line-width analysis } \\
\hline \hline buffer & \multicolumn{5}{|c|}{ 20 mM Sodium Phosphate } \\
\hline liposome composition & DMPC/DMPA 9:1 & DPPC/DPPA 9:1 \\
\hline $\mathrm{pH}$ & 6.5 & 6.0 & 7.0 & 6.5 \\
\hline \hline peak no. & \multicolumn{5}{|c|}{ line-width [ppm] } \\
\hline 1 & 0.75 & 0.75 & 0.66 & 0.90 \\
\hline 2 & 0.54 & 0.48 & 0.54 & 0.89 \\
\hline 3 & 0.68 & 0.70 & 0.88 & 0.95 \\
\hline 4 & 0.57 & 0.67 & 0.78 & 0.57 \\
\hline 5 & 0.58 & 0.62 & 0.81 & 0.91 \\
\hline 6 & 0.67 & 0.75 & 0.79 & 1.06 \\
\hline 7 & 0.59 & 0.55 & 0.65 & 0.79 \\
\hline
\end{tabular}

Table 3.5: ${ }^{15} \mathrm{~N}$ line-width analysis of the different samples measured with proton detected solid-state NMR. The values are reported in ppm. The peak no. is shown in figure 3.13 . 


\subsection{3 $\quad{ }^{1} \mathbf{H}$ detected solid state NMR}

With the the conditions obtained from the sample optimization, ${ }^{2} \mathrm{H} /{ }^{13} \mathrm{C} /{ }^{15} \mathrm{~N}$ samples of CitApc C12AR93A were produced for ssNMR both in the citrate-free and citrate-bound state. The hNH spectra of CitApc C12AR93A with and without ligand is reported in figure 3.14. The spectral quality in the absence of the citrate is lower and only 11 isolated peaks can be distinguished, in contrast with the 24 isolated peaks of the citrate-bound sample. Beside the advantages of the ${ }^{1} \mathrm{H}$ detected solid state NMR previously discussed (see section 1.6), the spectra of the transmembrane CitApc construct now share all the dimension with the spectra of the isolated CitA PASp and PASc domains and can therefore be directly overlaid, as done in figure 3.14 with the ${ }^{15} \mathrm{~N}-\mathrm{HSQC}$ of the globular domains and the hNH of the CitApc. In this way the presence of at least one outlier per sample is noticed immediately and is a good starting point for transmembrane assignments or, alternatively, for detecting differences between the structure of the globular domains in solution and in the membrane-bound construct.

For the citrate-bound sample, 3D spectra for backbone assignments were recorded (see table 2.4) and it was possible to assign 109 residues of the periplasmic domain and 32 of the cytosolic domain, for a total of 141 amino acids out of 298 expected and corresponding to $47.3 \%$ of the whole CitApc construct.

Most of the assigned residues are located in regions that form defined secondary structural elements in the crystal structures of the globular domains, with the amino-acids found in $\beta$-strands showing in general a higher peak intensity than the ones in $\alpha$ helices in the 3D hCANH (see figure 3.15 top). The difference in the intensities between the periplasmic and the cytosolic domain is not as marked as already reported from Schomburg (2014). Eight residues from the N-terminal helix of the PASc domain, that was so far not visible in the ssNMR experiments, have been assigned. Therefore, the assignments missing in the cytosolic domain must be due to discrepancies in the chemical shift values measured in the solution and the ones of the transmembrane construct, as can also be speculated from the 106 isolated peaks found in the 3D hCANH that are not yet assigned (see table A.6). These last would also be numerous enough to potentially obtain some assignments of the TM helices. 


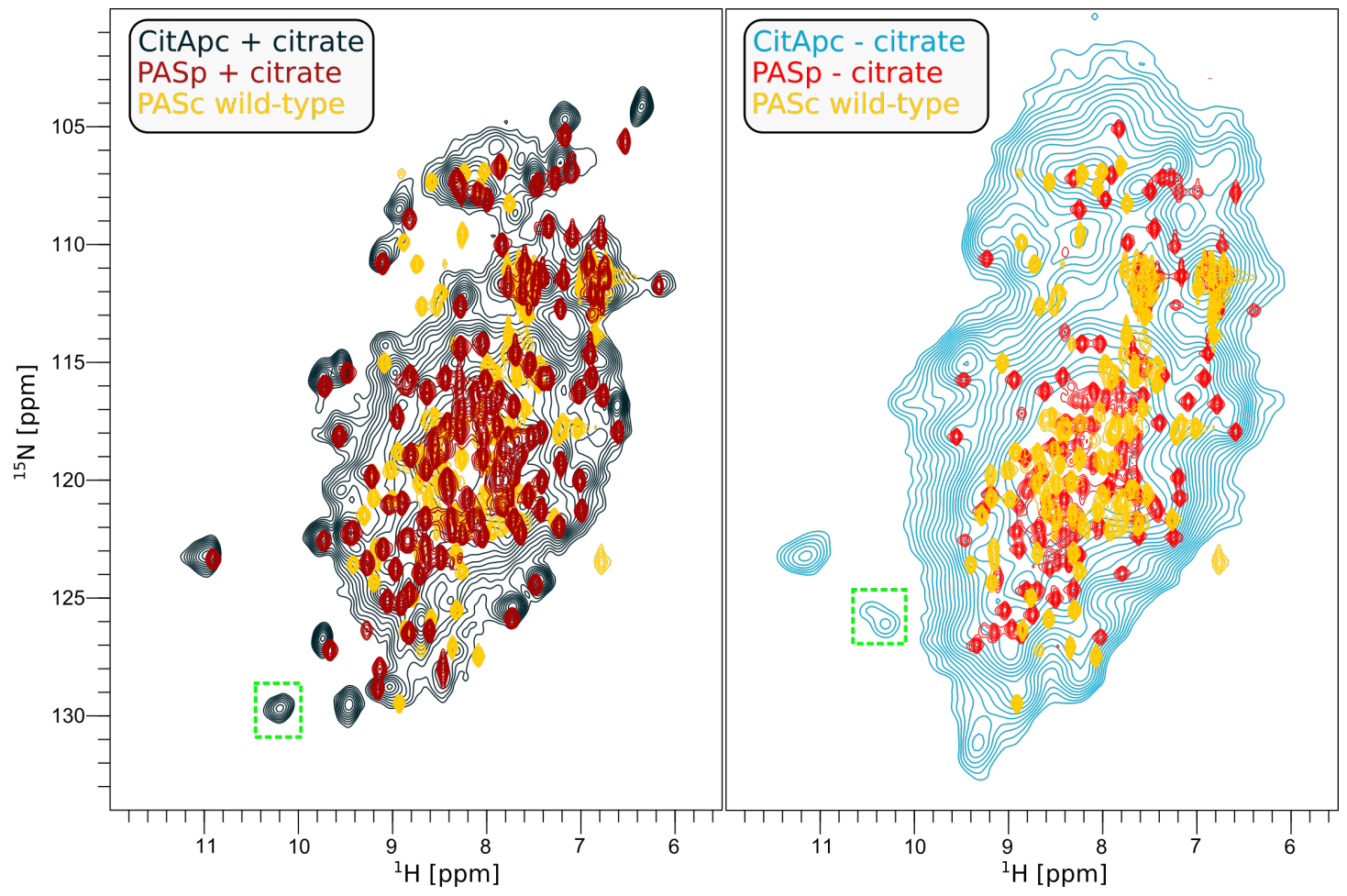

Figure 3.14: LEFT: The overlap of the solid-state hNH spectra of citrate-bound CitApc C12AR93A with the liquid-state ${ }^{15} \mathrm{~N}-\mathrm{HSQC}$ of citrate-bound PASp and the ${ }^{15} \mathrm{~N}-\mathrm{HSQC}$ of PASc wild-type. RIGHT: The overlap of the solid-state hNH spectra of citrate-free CitApc C12AR93A with the ${ }^{15} \mathrm{~N}$-HSQC of citrate-free PASp and the ${ }^{15} \mathrm{~N}-\mathrm{HSQC}$ of PASc wild-type. In the green boxes are highlighted two outliers that are only present in the CitApc samples and therefore either from the TM domains or resonances that shift between the isolated domain and the PASpc construct. The solid-state spectra were acquired at $55 \mathrm{kHz}$ spinning speed on a $850 \mathrm{MHz}$ AVANCE spectrometer equipped with a four-channel $1.3 \mathrm{~mm}$ MAS probe. The liquid-state spectra were acquired on a $800 \mathrm{MHz}$ AVANCE spectrometer equipped with a $5 \mathrm{~mm}$ triple-resonance cryogenic probe.

The secondary structure elements found in the crystal structures are also supported by the secondary chemical shift analysis (see figure 3.15 bottom). The positive value for leucine 155 is relevant for the triggering of the signalling relay as it extends what was already observed for leucine 154 and points to a more structured C-terminus in the bound state of the CitApc. In addition, it was possible to assign the periplasmic domain of the CitApc until the residue aspartate 157, which also show a positive secondary chemical shift.

The chemical shifts of the assigned residues were also compared to the chemical shifts measured for the globular domains using solution NMR. The shifts obtained for the CitApc were corrected according to the value reported by Maltsev et al. 


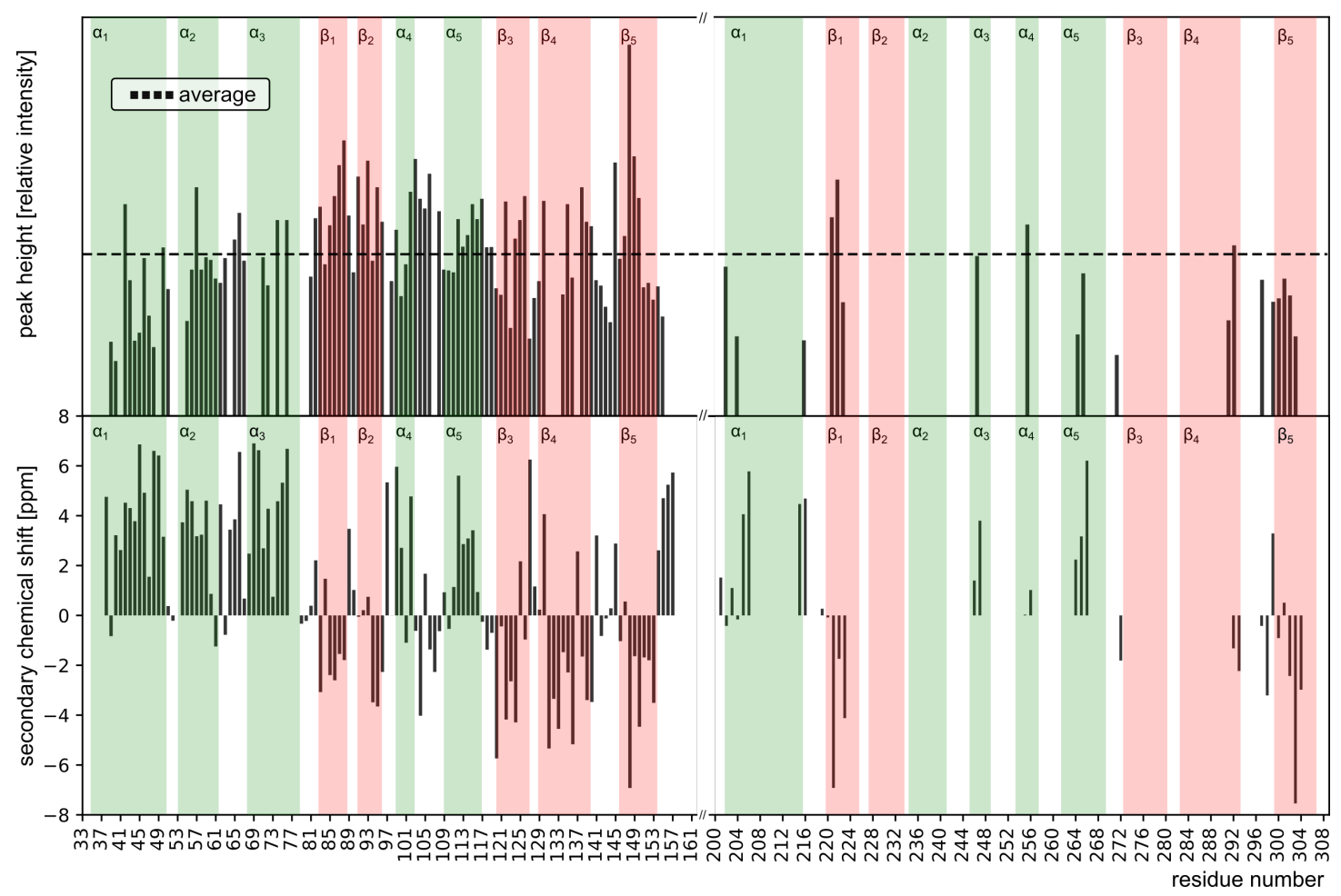

Figure 3.15: Top: peak heights of the assigned cross-peaks in the hCANH spectrum of the CitApc C12AR93A in the bound-state. Bottom: $\mathrm{C}_{\alpha}$ and $\mathrm{C}_{\beta}$ secondary chemical shift for the assigned residues.

(2012) for the intrinsically disordered protein $\alpha$-Synuclein to compensate the isotope effect introduce by the perdeuteration. Although also the secondary structure affects the size of the isotope effect (Maltsev et al., 2012), its contribution to the chemical is at least one order of magnitude smaller than the one generated from structural differences and reported previously for $G$. thermodenitrificans CitA (Salvi et al., 2017).

For the periplasmic domain, the chemical shift difference is within $0.5 \mathrm{ppm}$ for most of the residues (see figure 3.16 top), while the amino acids that exceed this value are found either at the dimer interface or at the periplasm-membrane interface (see figure 3.16). In the first case, the discrepancies are due to the absence of the dimer interface in the isolated PASp construct, which is found as monomer (Salvi et al., 2017). Instead, the very large differences at the C-termini involving residue glutammate 156 and aspartate 157 are a new finding and they are probably caused by the constraints that the membrane exerts on this part of the protein.

For the cytosolic domain, the chemical shift differences with the CitApc in the bound state were calculated not only with the PASc wild-type, but also with the PASc N288D and R289D mutants (see figure 3.17). A better agreement is obtained 

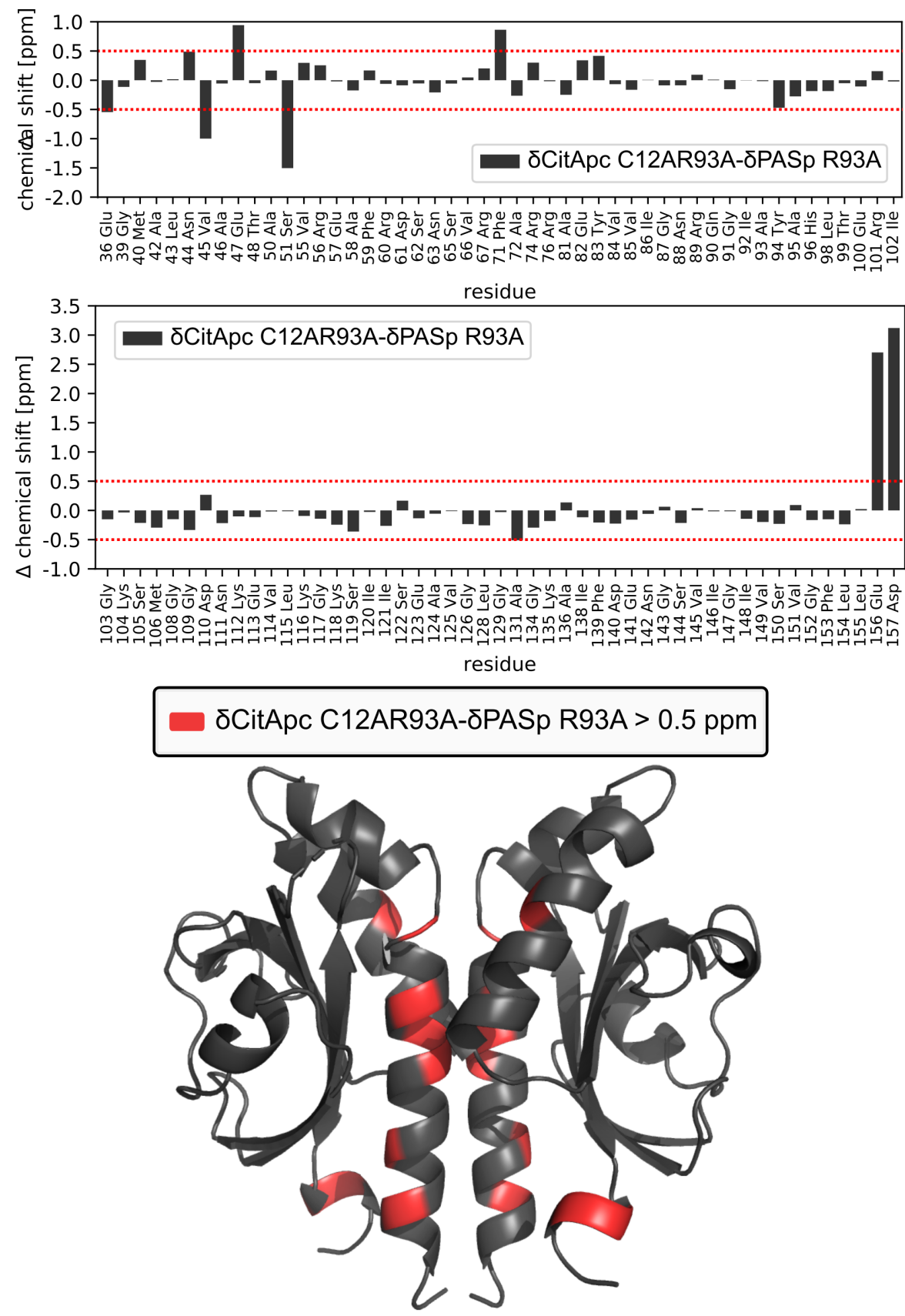

Figure 3.16: TOP: Differences between solid-state and solution NMR $\mathrm{C}_{\alpha}$ chemical shifts in the periplasmic domain. The chemical shifts obtained of CitApc were corrected to compensate the isotope effect. BOTTOM: the residues showing more than $0.5 \mathrm{ppm}$ difference reported on the citrate-bound PASp R93A crystal structure.

with the chemical shifts of PASc wild-type, especially in the case of residue alanine 216 and serine 304. The absolute value of the differences is in general larger than what observed for the periplasmic domain. In addition, residues that shows a discrepancy in the chemical shift bigger than $0.5 \mathrm{ppm}$ with the PASc wild-type do not cluster in any specific region of the protein and they are located in the 


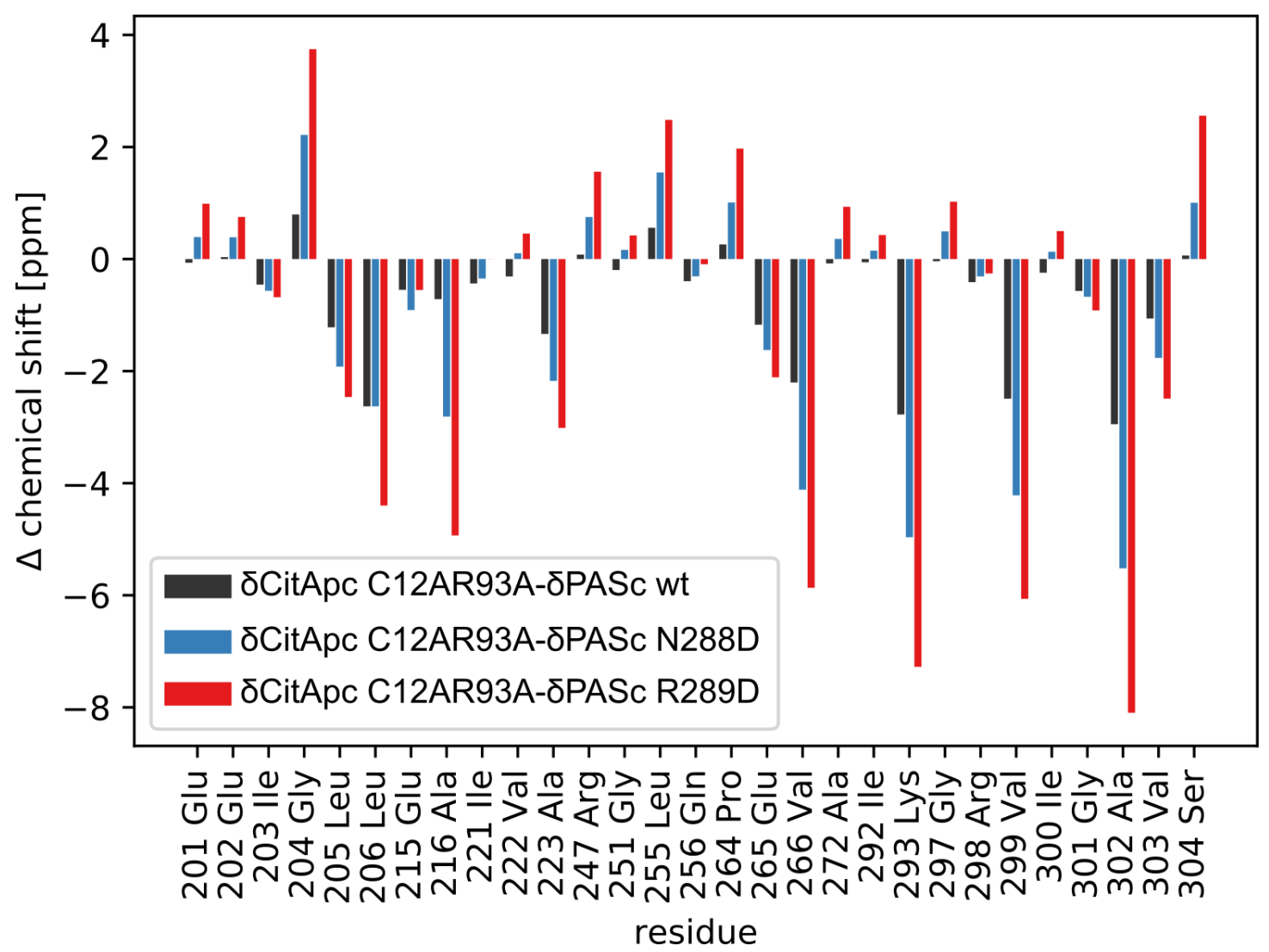

Figure 3.17: Differences between solid-state and solution NMR C $\alpha$ chemical shifts in the cytosolic domain. Perturbations are larger in the cytosolic domain than in the periplasmic domain. No clear offset caused by deuterium shift is distinguishable and the larger deviations are probably due to structural differences.

N-terminal helix, in the central $\beta$-scaffold and in the major loop.

Proton detected ssNMR experiments were also used to measure sPRE on the transmembrane construct in order to detect changes in the protein surface caused by the citrate binding event. As already reported for the isolated PASc samples, the intensity ratio between the doped and the reference sample, respectively $5 \mathrm{mM}$ and $0 \mathrm{mM}$ Gd-HP-DO3A, was used to evaluate the sPREs, but in this case a 3D hCANH spectrum was necessary to resolve as many isolated sites as possible. The effect of the paramagnetic agent is reported in figure 3.18. The reduction of the overall NMR signal estimated from the first FID is about $40 \%$. Nevertheless, the effect of the Gd-HP-DO3A is specific and it is reasonable to assume that the most effected peaks in fig 3.18 are the ones closer to the protein surface. Solvent PRE was evaluated for the citrate-bound CitApc using 106 isolated peaks in total, of which 79 were assigned to the periplasmic and 27 to the cytosolic domain (see table 3.6). The global average of the intensity ratios is 0.49 with a standard deviation 

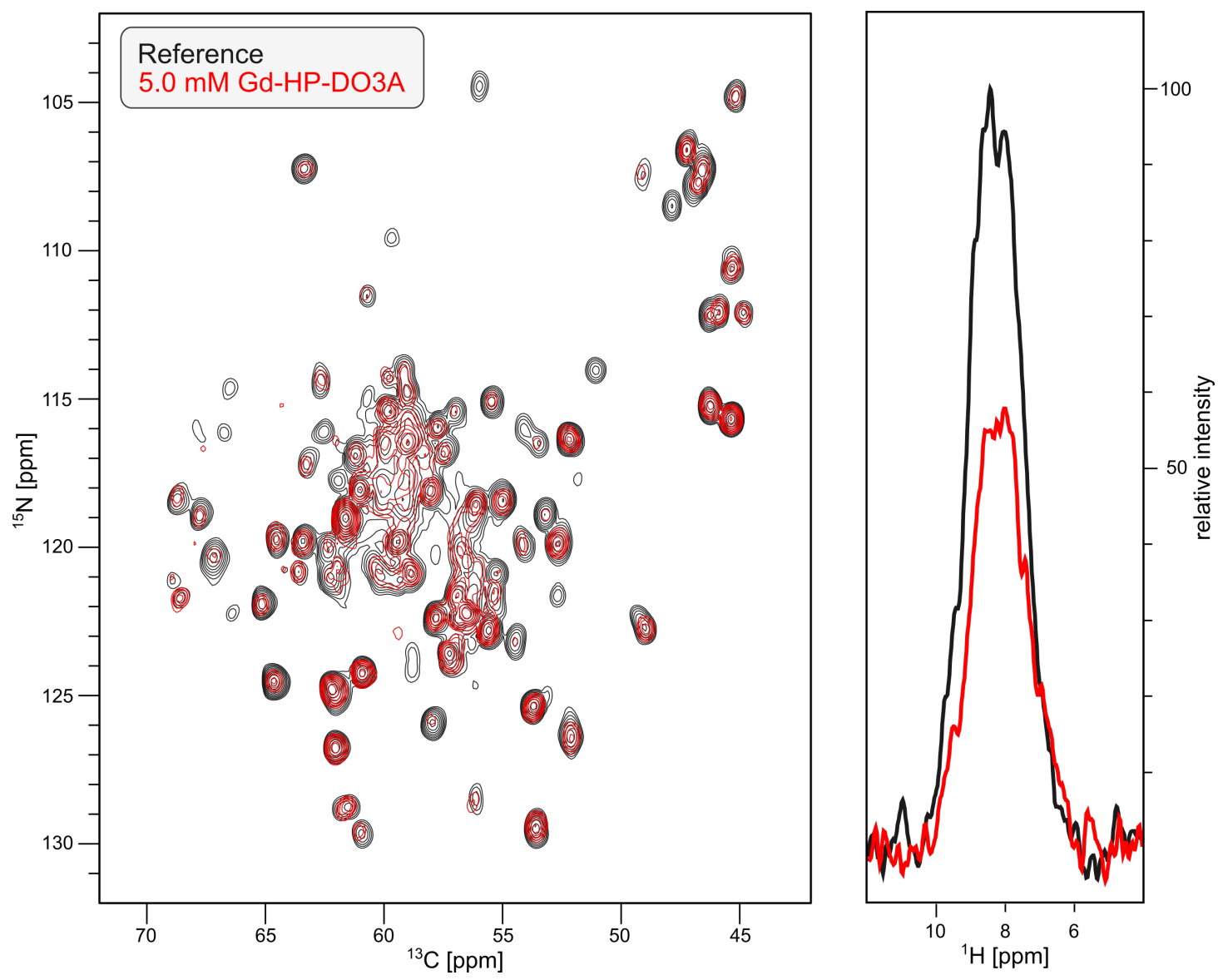

Figure 3.18: Effect of $5 \mathrm{mM}$ Gd-HP-DO3A on the 3D hCANH spectrum. From the first FID of the spectrum (right), a reduction of about $40 \%$ can be estimated for the 1D signal. From the ${ }^{15} \mathrm{~N}-{ }^{13} \mathrm{C}$ projection plane (left), it is possible to see that the signal loss is localized and that spectral quality is unaffected.

of 0.21. The average for the two different analysed domains is 0.54 for the PASp and 0.40 for the PASc with a standard deviation of 0.17 and 0.29 respectively. The experimental values, evaluated using the equation 2.9, are compared to the sPRE predicted using the algorithm developed by Öster et al. (2017), which gives as an output the slope of the linear dependence of R1 in function of the paramagnetic agent concentration, similarly to the approach adopted with the analysis of sPRE of the isolated PASc domain (see section 3.2). Using this procedure, a dip in the profile of the experimental $\mathrm{I}_{p} / \mathrm{I} d$ ratio should correspond to a peak in the predicted sPRE and vice-versa, if there is agreement between the experimental and predicted values.

For the PASp domain, sPRE were predicted on the crystal structure of citratebound $G$. thermodenitrificans PASp. A good agreement between experimental $\mathrm{I}_{p} / \mathrm{I}_{d}$ ratio and the predicted values is clear (see green, dashed boxes in figure 


\begin{tabular}{|l||c|c|c|}
\hline \multicolumn{1}{|c||}{} & \multicolumn{3}{c|}{ Citrate Bound } \\
\hline & Average & SD & No. of Peaks \\
\hline Global & 0.49 & 0.21 & 101 \\
\hline PASp & 0.53 & 0.17 & 76 \\
\hline PASc & 0.35 & 0.25 & 25 \\
\hline
\end{tabular}

Table 3.6: Statistics for the sPRE effects measured on the CitApc sample

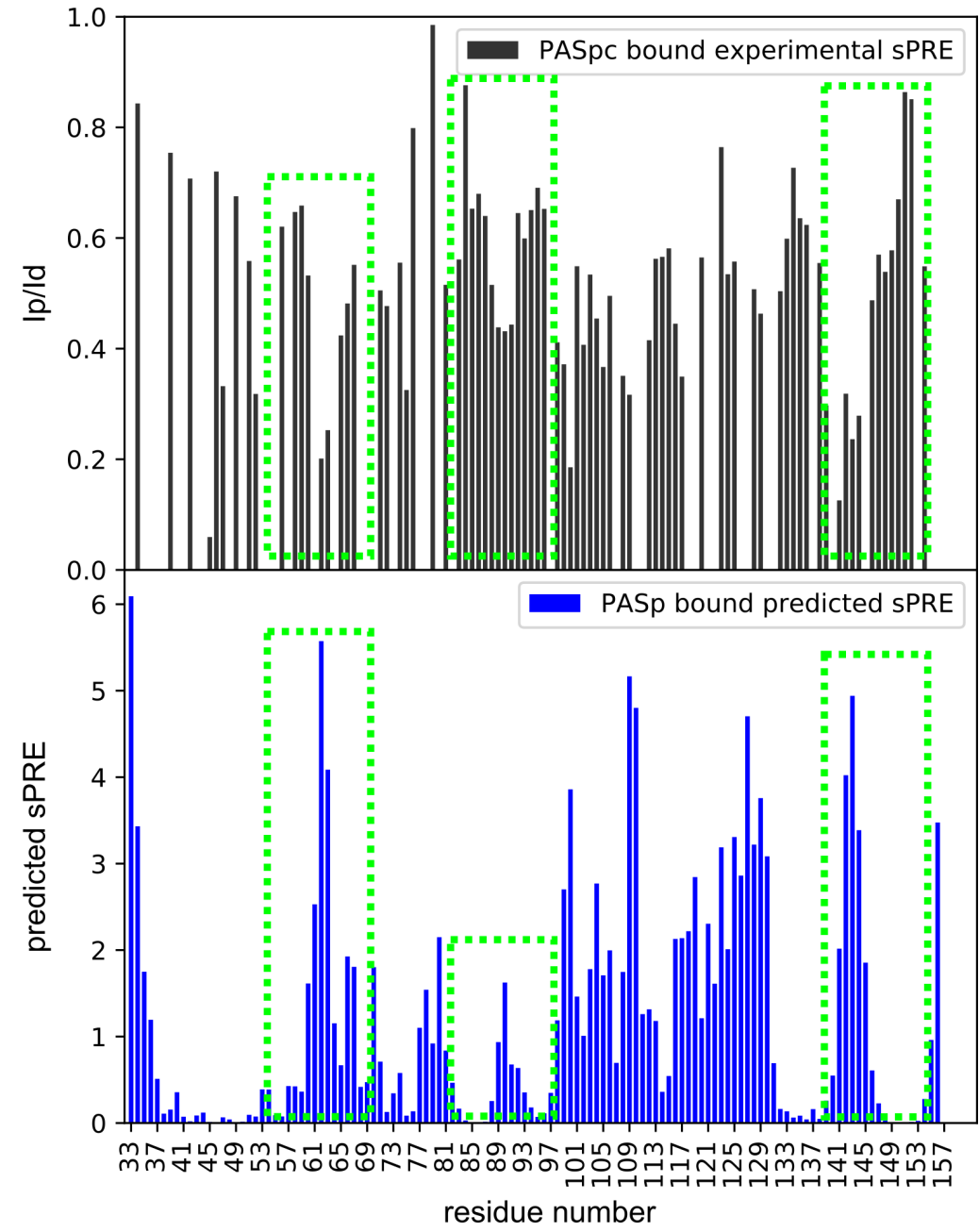

Figure 3.19: TOP: experimental sPREs of the periplasmic domain of CitApc. BOTTOM: predicted sPREs calculated on the G. thermodenitrificans PASp in the citrate-bound state. The green boxes highlight the similarities between the profiles.

3.19), although sPRE are evaluated for less than $70 \%$ of the PASp domain. For the cytosolic domain of the CitApc, sPREs were evaluated for less than $50 \%$ of the residues; thus, the comparison between the predicted and experimental values resulted impractical to discern which of the dimer arrangements is adopted in the bound state (see figure 3.20. In an alternative, qualitatively approach, the residues were categorized according to the value of their $\mathrm{I}_{p} / \mathrm{I}_{d}$ ratio as follows: 


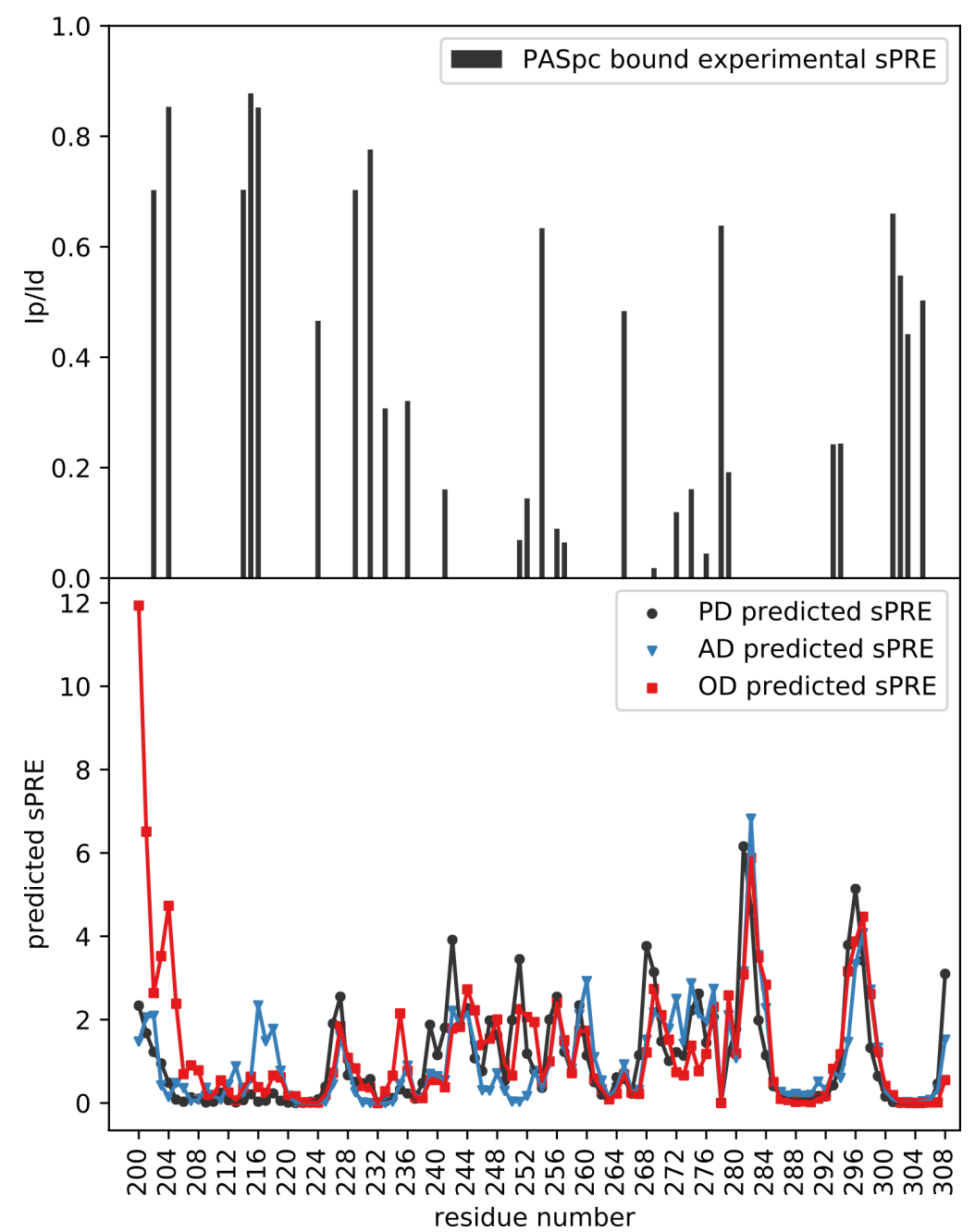

Figure 3.20: TOP: experimental sPREs of the cytosolic domain of CitApc. BOTTOM: predicted sPREs calculated on the parallel, antiparallel and open structures of $G$. thermodenitrificans PASc.

- not affected, with a ratio between 1.0 and 0.5

- partially affected, with a ratio between 0.5 and 0.2

- quenched, with a ratio lower than 0.2 .

The three groups were hence reported on the parallel, anti-parallel and open dimer structure for a qualitative analysis. Although less accurate than the comparison with the sPREs prediction, this procedure allows for preliminary analysis of the cytosolic domain of CitApc in the bound state. 


\section{Discussion}

\subsection{The periplasmic PAS domain}

The signalling relay of the $G$. thermodenitrificans CitA is initiated in the periplasmic PAS domain. Therefore, it is crucial to characterize the structural rearrangements caused by the citrate binding event in this domain to understand the signalling mechanism in its entirety. PAS domains are capable to bind a variety of small molecules, therefore they are often found as periplasmic receptor domains of transmembrane HKs. The binding mechanism of the periplasmic PAS receptor domain has already been characterized for DcuS (Cheung and Hendrickson, 2008) and for K. pneumoniae CitA (Sevvana et al., 2008). For G. thermodenitrificans CitA PASp, crystallization trials were unsuccessful at first, but the binding pocket was identified by chemical shift perturbation and the NMR-based structural models were solved for both citrate-free and citrate-bound states (Salvi et al., 2017).

To access the citrate free state of the PASp domain, the mutation R93A was necessary. This residue is highly conserved (Gerharz et al., 2003) and the corresponding arginine 66 in the PASp domain of $K$. pneumoniae is interacting directly with the ligand in the crystal structure of the citrate bound state (see figure 4.1). The mutation removes the polar interaction of the arginine side chain with the citrate and reduces the binding affinity, which would otherwise be too high to release the citrate (Schomburg, 2014).

Both the NMR-based citrate-free and -bound models of GtCitA

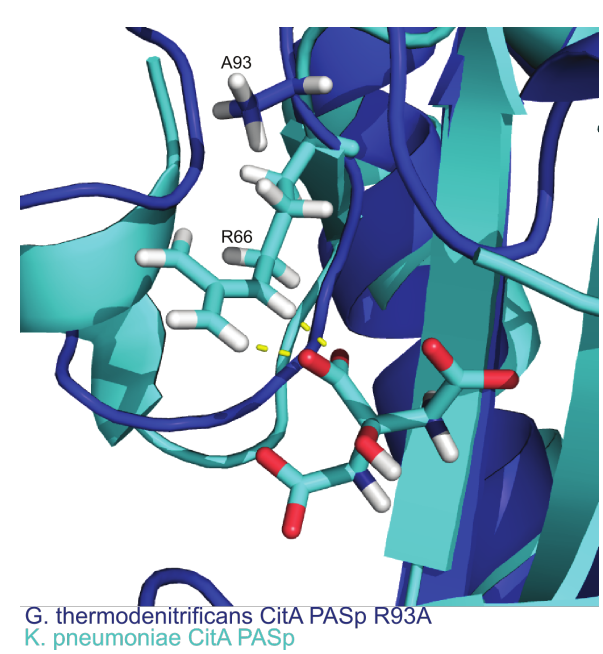

Figure 4.1: Structure alignment of the binding pocket of the GtCitA PASp (R93A) (in blue) with the crystal structure of KpCitA PASp (in cyan). The side chains of the mutated residue on the GtCitA PASp is shown together with the side chain of the conserved arginine in the KpCitA PASp. In yellow are represented the polar contacts between the citrate and the R66 sidechain of KpCitA.

PASp R93A agree very well with 
the crystal structures of the K. pneumoniae CitA PASp from Sevvana et al. (2008), with a backbone RMSD of $2.008 \AA$ and $1.957 \AA$ respectively (see figure $4.2 \mathrm{C}$ and $\mathrm{D})$.
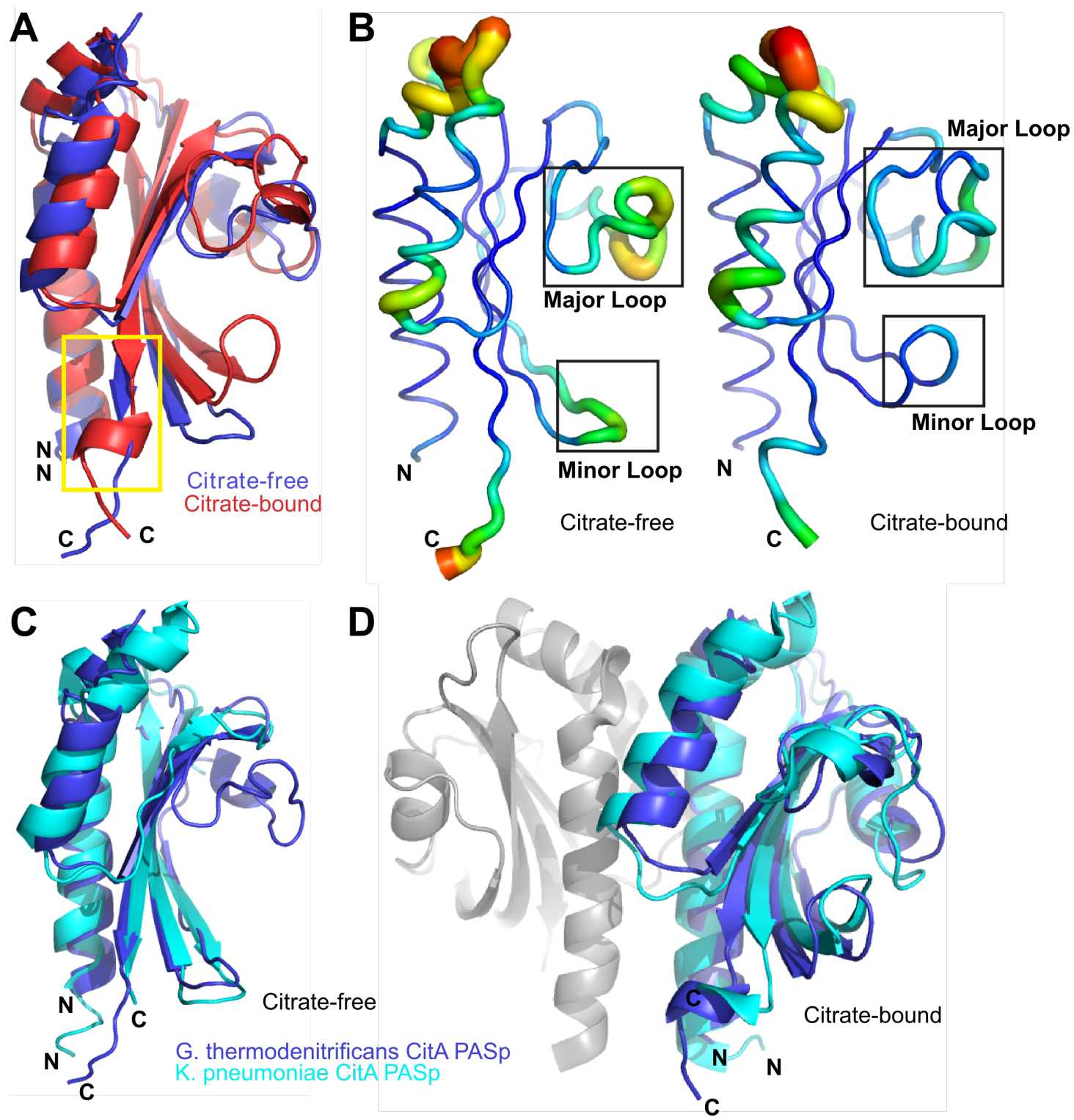

Figure 4.2: NMR-based structural model of CitA PASp (R93A). (A) Overlay of GtCitA PASp (R93A) structural models (ensemble of five lowest-energy structures) in citrate-free (blue) and citrate-bound (red) form. The shortening of the last $\beta$-strand is highlighted by the yellow box. (B) Structural disorder plot for the five lowest-energy structures in citratefree (left) and citrate-bound (right) states. Upon citrate binding, both the major- and minor loops (black boxes) closing the citrate binding pocket become more rigid. (C) Alignment of citrate-free GtCitA PASp (R93A) NMR model (blue) and Kp CitA PASp crystal structure (cyan) with a backbone r.m.s.d. of $2.008 \AA$. (D) Alignment of citrate-bound Gt CitA PASp (R93A) NMR model (blue) and Kp CitA PASp crystal structure (cyan) with a backbone r.m.s.d. of $1.957 \AA$. The second monomer of the Kp CitA PASp dimer is greyed out. 
Recently, the crystal structures of citrate-bound and of citrate-free $G$. thermodenitrificans CitA PASp R93A were solved. NMR models and crystal structures agree very well, with a backbone RMSD of $1.864 \AA$ for the bound state and of $2.931 \AA$ for the free state. In the latter, the higher RMSD is due to the major and minor loops opposite to the binding pocket, that are more flexible in absence of the ligand. Indeed, the RMSD value for the for the citrate-free structures drops to $1.368 \AA$ if only the ordered region are used for the calculation.

FREE

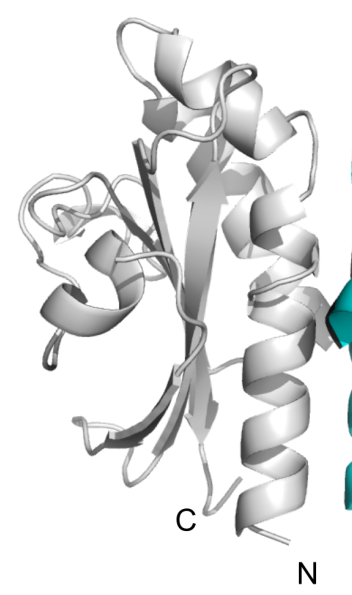

$\mathrm{N}$

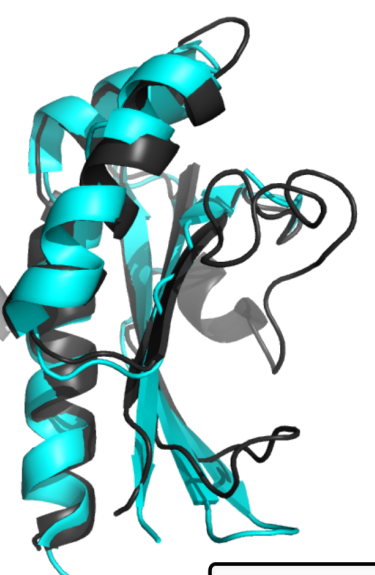

G. thermodenitrificans
K. pneumoniae
BOUND

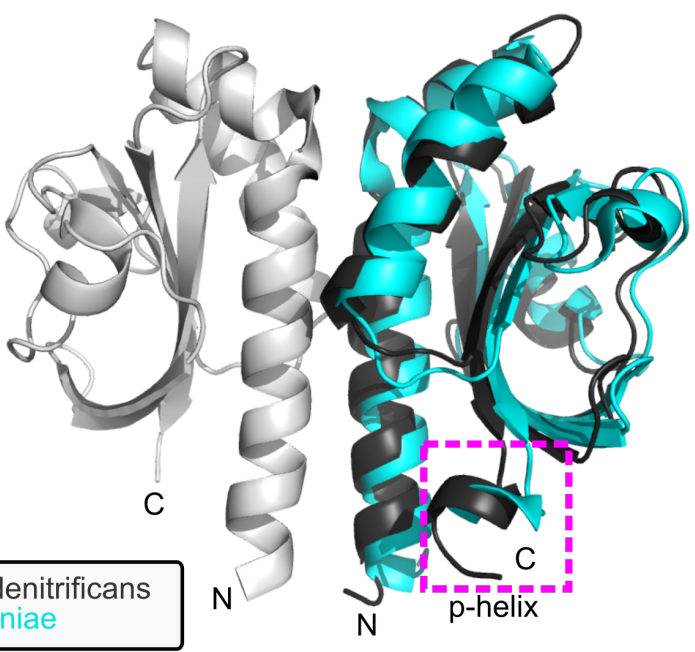

Figure 4.3: Alignment of the G.thermodenitrificans PASp (in black) with the K. pneumoniae PASp (in cyan) crystal structures for the citrate-free (on the left) and for the citrate-bound state (on the right). The second dimer subunit is greyed out. The C-terminal p-helix is highlighted by the pink, dashed box.

The crystal structure of $G$. thermodenitrificans CitA PASp also agrees with the one of K. pneumoniae CitA PASp (Sevvana et al., 2008), with an all-residue RMSD of $1.515 \AA$ and $1.004 \AA$ for the bound and for the free state respectively (see figure 4.3). Differences in the B-factor are present for both citrate-free and citrate-bound state (see figure 4.4). A lower mobility is observed for the G. Thermodenitrificans in the citrate-free state. In addition, also the major loop is solved also for the $G$. thermodenitrificans citrate-free CitA PASp, in contrast with K. pneumoniae citrate-free CitA PASp. In contrast to this, an higher mobility is observed in the G. thermodenitricans PASp structure for the citrate bound state.

The principal aspects of the binding mechanism are evident both in the NMR models and crystal structures of $G$. thermodenitrificans CitA PASp. The main feature is the contraction of the $\beta$-scaffold around the ligand (see figure 4.2 A). 


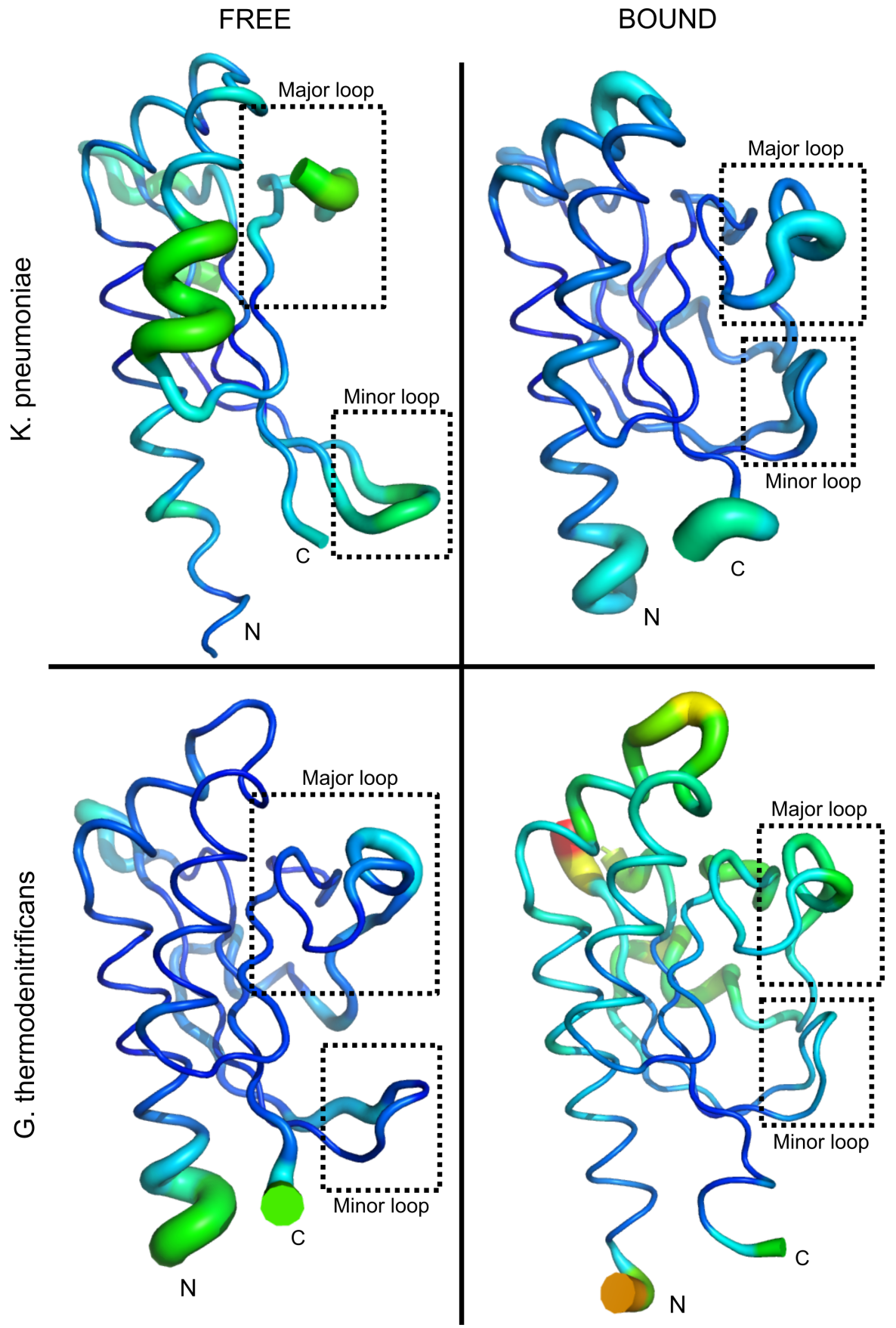

Figure 4.4: Disorder plot (B-factor) of the crystal structures of $G$. thermodenitrificans PASp and of K. pneumoniae PASp. Only the monomer unit is reported for each state and each organism. For the citrate-free state (on the left) the major loop is solved only for $G$. thermodenitrificans. In the citrate-bound state (on the right), the K. pneumoniae is overall less disordered (lower B-factor).

The effects of the contraction are propagated to the C-terminus, where a rearrangement of the secondary structure occurs. First of all, the switch of leucine 154 from a $\beta$-strand to an helix conformation is revealed by the secondary chemical shifts and it results in the shortening of the last $\beta$-strand by one residue (Salvi 
et al., 2017). Secondly, the formation of a short $\alpha$-helix from residue leucine 155 to residue aspartate 157 at the C-terminus is evident from the NMR model and from the crystal structure, although secondary chemical shifts show only a small propensity for this residues to adopt an helical structure due to the flexibility of the C-terminus (see figure 4.8).

In K. pneumoniae CitA PASp, both the contraction of the $\beta$-scaffold and shortening of the last $\beta$-strand are present (Sevvana et al., 2008). The latter occurs at residue threonine 128, which corresponds to the leucine 154 of $G$. thermodenitrificans CitA in the sequence alignment. The formation of the C-termini p-helix is present as well, at least on one of the dimer subunit, and it involves the residues corresponding to leucine 155, glutammate 156 and aspartate 157 of $G$. thermodenitrificans CitA. A C-terminal helical element is instead present in the ligand-bound structure of E. coli DcuS (Cheung and Hendrickson, 2008), where more residues following the last $\beta$-strand are solved (see figure 4.8).

Summarizing, the contraction of the central scaffold is confirmed in the isolated G. thermodenitrificans CitA PASp as the main feature of the binding mechanism. In addition, the shortening of the last $\beta$-strand the formation of the p-helix at the C-termini upon citrate binding may be the key aspects of the signalling mechanism from the periplasmic to the transmembrane domain, if they also occur in the context of the membrane.

\subsection{The cytoplasmic PAS domain}

The PAS fold is a very well known motif, but none of the deposited structures show a dimer arrangement different from the one in figure $3.5 \mathrm{~A}$. Although changes at the dimer interfaces have been identified as a possible mechanism for signalling to downstream domains for membrane proximal PAS domain of the double-cache receptor in DctB (Liu et al., 2014), it is still surprising that crystal structures of PASc mutants based on functionally relevant mutants in DcuS (Monzel et al., 2013) showed very different dimer arrangement. Samples of CitA PASc N288D and R289D were produced for liquid-state NMR spectroscopy to characterise alternative dimeric conformations in absence of crystal packing contacts. Like PASc wild-type and all the proposed CitA functional mutants, PASc N288D and R289D appear to be a dimer in solution (see figure 3.3) Differences between the samples 
can already be observed from comparison of the $2 \mathrm{D}{ }^{15} \mathrm{~N}-\mathrm{HSQC}$ spectra (see figure $4.5)$.

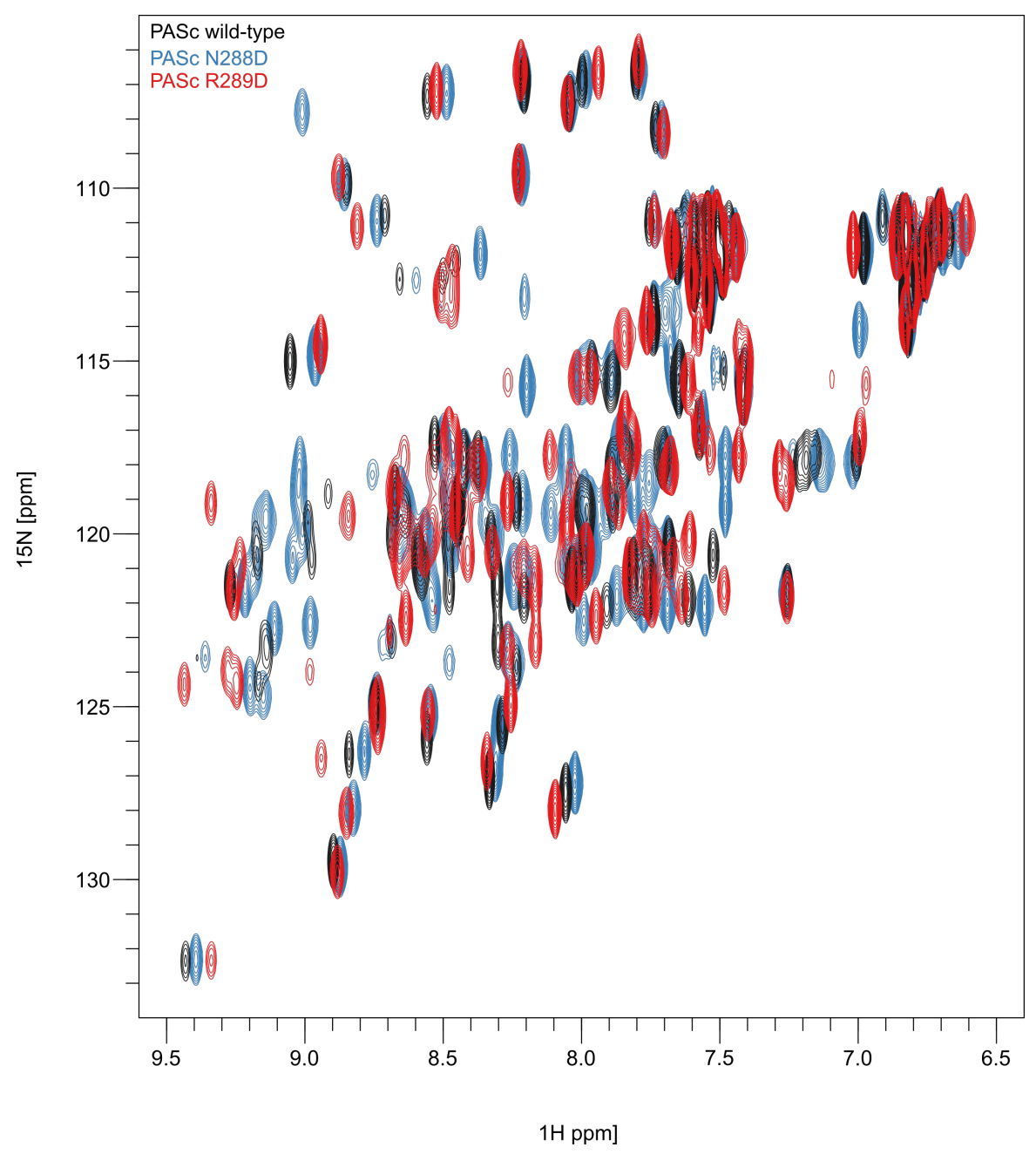

Figure 4.5: Overlap of the ${ }^{15} \mathrm{~N}$ HSQC of the PASc wild-type (black), PASc N288D (blue) and PASc R289D (red).

After sequential assignment, carbon chemical shifts were used to localize the differences down to single amino acid resolution. If residues sequentially or spatially close to the mutation site are excluded, the only significant differences are located in the N-terminal $\alpha$-helix (see figure 3.6), which is also the only secondary structure element undergoing significant changes (see figure 4.6) The changes in the chemical shifts reveal that the PASc mutants differ from the wild-type not only in crystals, but also in solution. However, shifts are not sufficient to assert that the PASc mutants domains adopt the alternative dimer conformations observed in the crystals.

The alternative dimer arrangements were also investigated using sPREs, which are 
a very powerful tool to study protein surfaces and their changes upon intermolecular interaction (Pintacuda and Otting, 2002). The characterization of the PASc dimer interface was not so straightforward as the differences in the predicted sPRE values are mainly located in the N-cap helix, where some assignment is missing. Nevertheless, it is possible to assert from the analogies between the experimental and predicted value (see figure 3.8) that at least the anti-parallel conformation is adopted by the PASc N288D. The distinction between the open and parallel dimer is not so clear because the differences in the predicted sPRE at the N-terminus are mainly due to the disorder present in the crystal structure of the open dimer in this region. In collaboration with the group of Prof. Sandoghar, the inter
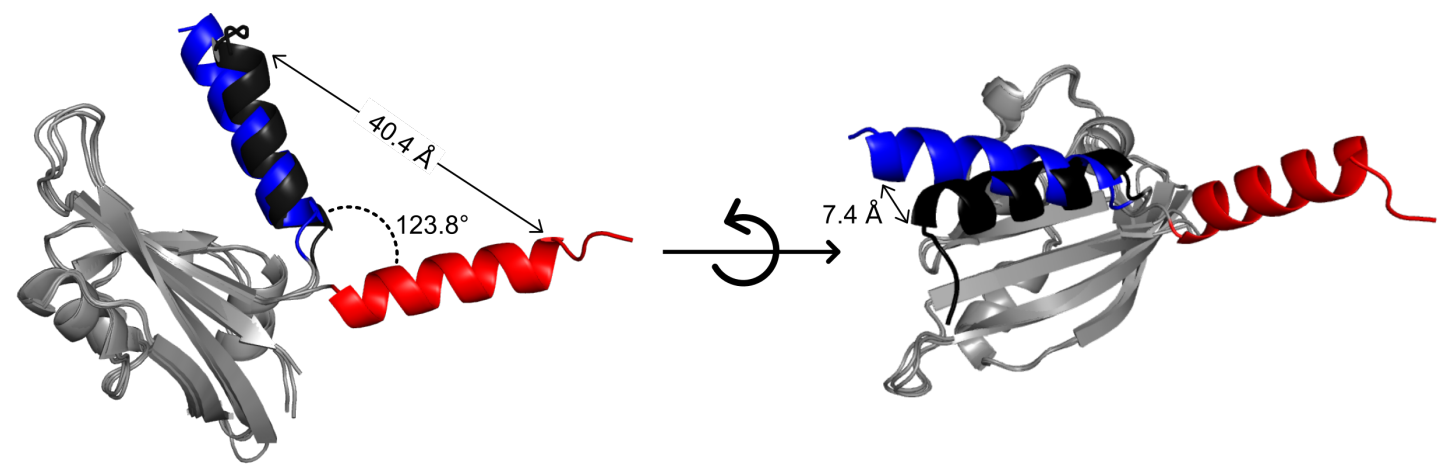

Figure 4.6: Alignment of the monomer from the crystal structure of the PASc: wild-type (N-terminal helix in black), of the PASc N288D (N-terminal helix in blue) and of the PASc R289D (N-terminal helix in red). The core of the PAS domain is represented in grey.

unit distance between the C-termini was measured using COLD (Weisenburger et al., 2017). The experimental values agree with the distances calculated from the crystal structures (see table 3.3), confirming that the non wild-type CitA PASc domains can adopt dimer arrangements different from the one observed so far for this kind of fold.

Dimer interfaces were further characterized using NMR. NOESY experiments are ideal to detect inter unit contacts as cross peaks arise from cross-relaxation of spins that are closer than $7 \AA$. Additionally, to isolate the cross peaks generated at the dimer interface, a double-edited 13C-15N 3D-NOESY experiment (Kay et al., 1990) was measured on a sample where a 13C labelled protein was mixed in a 1:1 ratio with a $15 \mathrm{~N}$ labelled protein. The observed contacts were assigned based on chemical shift matching and interpreted based on the distances calculated from the crystal structures. If the cut-off for a reliable NOESY cross-peak is set to $7 \AA$, it is not possible to assert that a defined dimer arrangement is adopted only in one 
sample (see figure 4.7), in contrast to observed in the crystal structures and in the COLD measurements. Then it is necessary to assume that, under the conditions
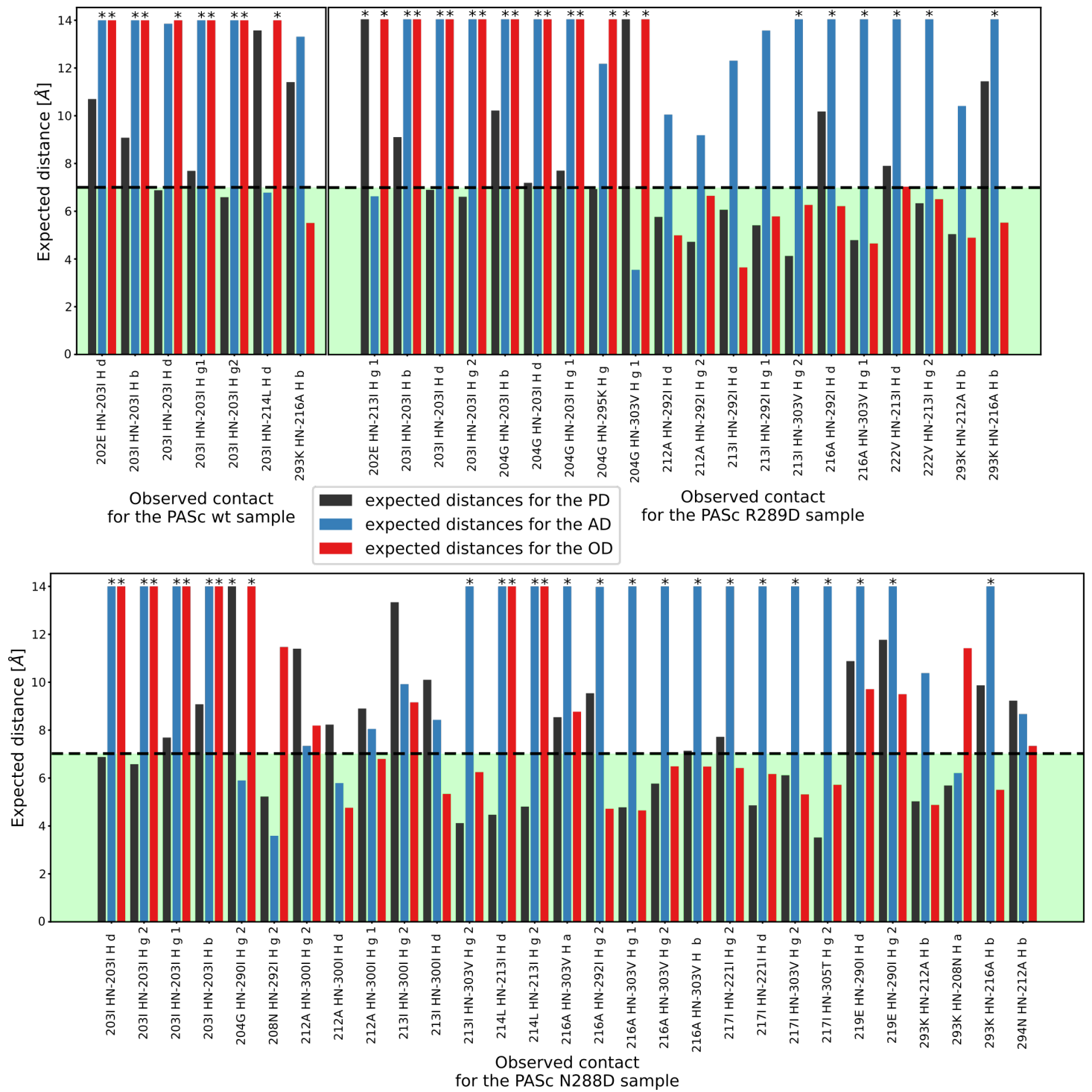

Figure 4.7: Analysis of the 13C-15N double edited NOESY measured on the PASc wild type (A), on the PASc R289D (B) and on the PASc N288D (C). The inter-unit distances calculated for the crystal structures are reported in black for the parallel dimer arrangement (PD), in blue for the anti-parallel (OD) and in red for the open dimer (OD). The green box emphasizes all the distances that are shorter than $7 \AA$ in the three dimer conformations previously showed. To explain all the assigned cross peaks, it was necessary to assume that a certain sample is capable of adopting multiple dimer arrangement.

used for the NMR experiments (see section 2.1.2), a certain sample can adopt more than a single dimer arrangement. With these assumption, it was possible to explain all the cross-peaks found in the spectra and it can be concluded that each sample is able to adopt at least two different arrangement and in particular: 
- CitA PASc wild type implements the PD state but at least AD is present.

- CitA PASc N288D implements the AD state but at least PD is present.

- CitA R289D implements the OD state but both AD and PD are present.

Another proof of the capability of the CitA PASc to adopt multiple dimer arrangements came from the result of the CEST experiments. These are capable to detect low populated excited states that are in slow exchange with the ground state (Vallurupalli et al. (2017)). In the case of CitA PASc, CEST experiments detected a second and low populated conformation for the wild-type, as well as for the N288D and for the R289D mutants. The residues that exhibited exchange belong to secondary structural elements involved in the dimer formation, namely the first $\alpha$-helix, the fourth and fifth $\beta$-strands and the major loop (see figure 3.10). Interestingly, the latter is involved in inter-unit contacts only in the anti-parallel dimer arrangement, but it shows exchange in all the three samples. As already discussed previously (see section 3.2), the three dimer conformations differ only in the orientation of the N-terminal helix. Therefore, based on the location of the residues showing exchange in the $15 \mathrm{~N}$ CEST profile, the detected exchange is indeed the one between different arrangements, supporting what was found also from the double-edited 13C-15N 3D-NOESY experiment.

Two different scenarios are then found by NMR experiments (double-edited NOESY and CEST), and the other techniques exploited (crystallography and COLD)(see table 4.1). In these latter, the dimer is static and only one conformation per sample is found. In the former, the dimer arrangement is mutable, with different conformations that coexist in the same sample with variable populations. These alternative situations are not mutually exclusive and can be easily explained. Indeed, COLD measurements are not sensitive to lowly-populated state, while crystals cannot accommodate too many different conformations.

Concluding, all the evidences reported so far point out that the CitA PASc is indeed capable to adopt different dimer conformations and they are not just caused by crystal packing, but observed also in its absence. This conclusion is also backed up by Etzkorn et al. (2008), who investigated the isolated cytosolic domain of the E. Coli DcuS, a close homologue of CitA, using solid-state NMR and structure modelling and reported an intrinsic flexibility of the whole domain. In particular, the modelled structure of the DcuS in the monomeric state showed that the N-cap helix can adopt different orientations in the modelled structure. This statement can 


\begin{tabular}{|l|l|c|c|c|}
\hline Sample & Technique & Parallel & Anti-parallel & Open \\
\hline \multirow{4}{*}{ Wild-type } & Crystallography & + & - & - \\
\cline { 2 - 5 } & COLD & + & - & - \\
\cline { 2 - 5 } & double-edited NOESY & + & + & \pm \\
\cline { 2 - 5 } & CEST & + & + & + \\
\hline \multirow{4}{*}{ N288D } & Crystallography & - & + & - \\
\cline { 2 - 5 } & COLD & - & + & - \\
\cline { 2 - 5 } & double-edited NOESY & + & + & \pm \\
\cline { 2 - 5 } & CEST & + & + & + \\
\hline \multirow{4}{*}{ R289D } & Crystallography & - & - & + \\
\cline { 2 - 5 } & COLD & - & - & + \\
\cline { 2 - 5 } & double-edited NOESY & + & + & + \\
\cline { 2 - 5 } & CEST & + & + & + \\
\hline
\end{tabular}

Table 4.1: The dimer conformations found for each sample and divided by technique. The plus represents an arrangement that has been detected for the sample with that technique. The minus represents an arrangement that has not been detected for the sample with that technique. The \pm symbol is used to indicate ambiguous results.

now be extended, deducing that the orientations of the N-terminus are responsible for alternative dimeric conformations. In a crystal, the alternative arrangements represent an energy minimum and only one structure is found in each sample of CitA PASc (e.g. parallel dimer in the PASc wild-type, anti-parallel dimer in the PASc N288D and open dimer in the PASc R289D). In solution, the restraint of the crystal packing is not present and every dimeric conformation exists with a population level proportional to its energy and exchange with the other possible conformations.

Still, the question of whether these structures are relevant for the signal transduction remains open and has been investigated using the transmembrane construct CitApc via solid-state NMR. 


\subsection{Signalling relay in the $G$. thermodenitrifi- cans CitA}

\subsubsection{Citrate binding and activation of the PASp in con- text of the membrane}

The citrate binding mechanism of $G$. thermodenitrificans R93A PASp has been very well characterised for the isolated domain and discussed previously (see section 4.1). Following the chemical shift perturbations, it was possible to show that the structural changes in the isolated PASp domain upon citrate binding are also present in the PASpc construct. In addition to the published results (Salvi et al., 2017), the secondary chemical shift can be followed up to residue aspartate 157 for the citrate bound CitApc (see figure 4.8). The secondary chemical shifts of the isolated, citrate-bound PASp R93A correlates very well with the one of the citratebound CitApc C12AR93A. However, differences in the values are present after the residue leucine 154 , with the transmembrane construct showing a much stronger tendency to adopt an helical structure. Thus, the C-terminus of the periplasmic domain is constrained in the CitApc construct by the presence of the TM helices and a well defined helical element is present only in citrate-bound CitApc.

The C-terminal $\alpha$-helix of the periplasmic domain is nevertheless present both in the NMR model and in the crystal structure of citrate-bound PASp, which then agrees with the secondary chemical shifts of the citrate-bound PASpc up to the residues aspartate 157 .

Therefore, the similarities between the isolated receptor domain and the transmembrane construct go beyond leucine 154. In analogy to the NMR models of figure $4.2 \mathrm{~A}$, it can be concluded that in the transmembrane construct the structure of the C-terminus of the periplasmic domain is not well defined in the absence of the ligand. Then, upon citrate binding, the last residues on periplasmic side of the membrane form an $\alpha$-helical structure, which may continue almost uninterruptedly until TM2.

Moreover, secondary chemical shifts indicate a sudden change in the secondary structure and the transition from $\beta$-strand to $\alpha$-helix occurs between residue 153 and 155 almost without any loop. A rapid conversion of the secondary structure is present in the crystal structure and NMR model of CitA PASp as well between phenylalanine 153 and leucine 155, in very good agreement with the secondary 


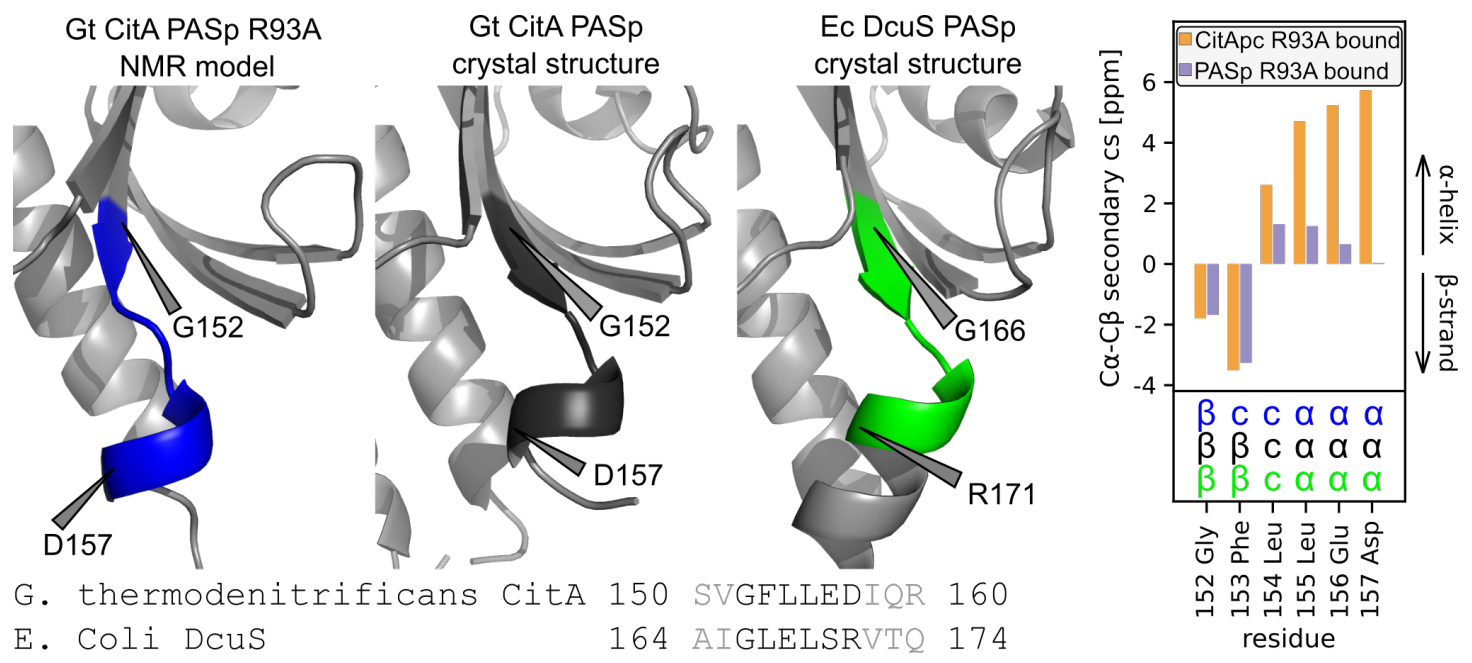

Figure 4.8: C-termini of the citrate-bound CitA PASp R93A NMR model (blue), of the citrate-bound CitA PASp crystal structure (black) and of the fumarate-bound DcuS PASp crystal structure (green). On the bottom, the alignment between $G$. thermodenitrificans CitA and E. coli DcuS is showed only for the residues highlighted in the structures above. On the right, the $\mathrm{C}_{\alpha}-\mathrm{C}_{\beta}$ secondary chemical shifts measured for citrate-bound CitApc C12AR93A (in orange) and for the citrate-bound PASp R93A (in purple) together with the secondary structure extracted from the displayed structures. The differences between the secondary chemical shifts of the transmembrane construct and of the isolated domain are evident only after the residue leucine 154. On the bottom, the alignment between the C-termini of $G$. thermodenitrificans CitA PASp and E. coli DcuS PASp.

chemical shifts. The sudden switch at the periplasmic C-termini is not specific of CitA, but it is also present in E. coli DcuS PASp (Cheung and Hendrickson, 2008). In DcuS, the conversion of secondary structure from $\beta$-strand to helix occurs between residues leucine 167 and leucine 169, which align with the aforementioned residues of CitA (see figure 4.8).

Thus, the p-helix (see section 1.3.2) is also found in the ligand-bound state of receptor PAS domains and might be essential for the relay of the signal from the sensor domain to the TM helices also in G. thermodenitrificans CitA PASp, adding to the hypothesis of a common mechanism for the triggering of HKs (Bhate et al., 2015).

\subsubsection{The transmembrane region}

The transmembrane domain of the CitApc construct was so far only visible in the citrate bound state when the protein was reconstituted in asolectin (Salvi et al., 2017). In an attempt to reduce the crowding of the spectra and improving the 
assignment of the TM helices, CitApc samples were washed in deuterated buffer. This approach led to a signal reduction of about one third, which made it impossible to acquire 3D experiments for sequential assignment, but it also resulted in a less crowded NCA plane (see figure 3.11).

With this strategy, three residues located in the transmembrane region were assigned based on the available chemical shifts for CitApc embedded in asolectin liposomes. The chemical shifts of both assigned peaks is indicative of an helical conformation (Wang and Jardetzky, 2002), but the differences between the free and the bound state for these residues are not significant (see figure 4.9), suggesting no major changes in the secondary structure between the OFF and the ON state of CitApc for these amino acids. The ligand binding event is however not supposed to trigger major changes in the secondary structure of the TM domain. In DcuS, a cysteine accessibility approach was used by Monzel and Unden (2015) to study the TM region signalling mechanism and only a piston-like displacement of the TM2 was observed, without any particular influence on the helical conformation.

Many more residues of the transmembrane domain were expected to be assigned
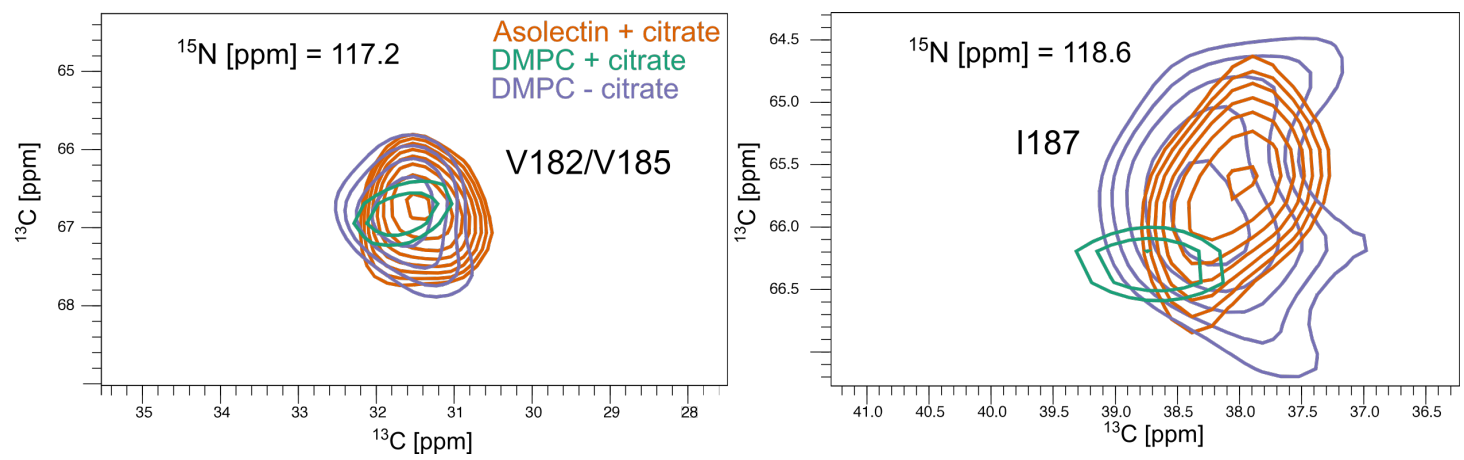

Figure 4.9: Overlap of the cross-peaks used for the assignment of the TM2. In black the signal of the holo-CitApc in asolectin, in light blue the signal of the holo-CitApc R93A bound in DMPC and deuterated buffer and in orange the signal of the apo-CitApc R93A in DMPC and deuterated buffer.

in the sample washed with deuterated buffer as the amino acids embedded in the liposome are expected to be by far less exposed to the solvent than those in the globular domains. Despite this, many factors can explain the limited assignments. One of these can be intrinsic structural heterogeneity of the TM helices. Indeed, flexibility is believed to be a desired feature when coming to signalling relay (Gushchin and Gordeliy, 2018). Most of the structural information about the TM helices of HKs is developed from indirect data, such as cysteine cross-linking 
(Hughson and Hazelbauer, 1996; Monzel and Unden, 2015) and physiological studies of mutant proteins, and few high resolution data available (Gushchin et al., 2017; Rieping et al., 2007). In particular, Gushchin et al. (2017) have reported that the transmembrane signalling of NarQ is best described by a combination of piston shifts, diagonal scissoring and twisting. This tangled picture is further complicated by the asymmetric structure of the holo-NarQ, which introduces skewing and asymmetric helical rotation (Gushchin et al., 2017). This variety of motions can cause structural heterogeneity that will result in severe line broadening of the NMR signals, making them disappear below the noise leveland if a distinct conformation is not isolated in the sample.

Another reason for the low visibility of the transmembrane domain might be the permeability of the membrane. Although the residues embedded in the liposomes are less exposed to the solvent, it is still possible for water molecules to cross the membrane even without any obvious channel. Actually, the four helix bundle formed by the dimeric state of HKs might create a small pore accessible to water, as found in the crystal structure of NarQ (Gushchin et al., 2017). As a result, the exchange of the amide proton of the residues buried in the membrane is less likely, but it can not be excluded. A third explanation of the scarce visibility of the TM region can be given based on the assignment approach. Indeed, spectra for the sequential assignment could not be acquired after soaking the samples with deuterated buffer due to low signal-to-noise ratio. So it is possible that NMR signal from the TM region is not washed out, but it cannot be either resolved nor assigned based on the acquired experiments (see 2.3). This is especially true when talking about helical structure elements, where the chemical shift dispersion tends to be smaller than in $\beta$-sheet.

\subsubsection{The role of the cytosolic PAS domain in the CitApc construct}

After proving that the different dimeric conformations exist and exchange between each other in the isolated PASc domain, the hypothesis that one or more of these dimer arrangements of the PASc are relevant for the signalling to the down-stream kinase domain is more concrete than previously assumed (Schomburg, 2014). To further investigate this hypothesis, overall visibility, chemical shifts, deuterium 
exchange patterns and sPREs were used to compare the PASc samples with the transmembrane construct in the citrate-bound and in the citrate-free state.

Overall visibility Differences in the overall visibility of the cytosolic domain of CitApc upon the citrate binding supported the signalling mechanism based on overall destabilization of the PASc domain. Indeed, it was observed that the overall visibility of the cytosolic domain of CitApc went from $59 \%$ to $45 \%$ upon the binding event and that at least 12 of the $\mathrm{C}_{\alpha}-\mathrm{C}^{\beta}$ cross-peaks were visible only for citrate-free PASpc (Salvi et al., 2017). In addition, these results were corroborated by functional studies on $\mathrm{HK}$ ArcB, where formation of disulfide bridges, i.e. reduced flexibility, between two PAS-monomers preceding the DHp domain switches off the kinase activity (Malpica et al., 2004). Moreover, increased flexibility was proposed as kinase activity switch also for E. coli DcuS (Etzkorn et al., 2008).

Still, the proposed overall destabilization mechanism does not rule out possible dimer rearrangements, such as the ones observed in the crystal structures of CitA PASc, and the changes in the dynamics might be coupled with a structural reorganization. Exploiting the assignment of CitA PASc N288D and CitA PASc R289D, it is possible to compare the overall NMR visibility of the isolated PASc and of its mutants with the citrate-free and citrate-bound state of CitApc. The backbone assignment of wild-type PASc reached only $86 \%$ completeness, in contrast to PASc R289D and PASc N288D, which reached 93\% and 95\% completeness, respectively. This suggests that the bound-state of the CitApc, in which the visibility of the cytosolic domain was lower, is similar to the PASc wild-type, which adopt predominantly the parallel arrangement. Similarly, the visibility of citrate-free CitApc resembles the one of the PASc N288D, which is found mainly in the antiparallel conformations. Thus, it can be proposed that a structural reorganization of the cytosolic PAS dimer arrangement accompanies the destabilization induced by the citrate binding. The more stable anti-parallel arrangement is adopted in the OFF-state of the cytosolic PAS domain, while the flexible parallel arrangement is found in the ON-state. In this scenario, the open dimer could represent a low-populated, non-detectable transition state or, alternatively, it could only be adopted in the isolated PASc.

Chemical shift comparison As already discussed (see section 3.2), differences in the $\mathrm{C} \alpha$ chemical shifts between the PASc mutants and the PASc wild-type were 
observed only in the N-cap helix and in the region sequentially and spatially related to the N288D or R289D mutation. Therefore, the ssNMR assignment of the same helix becomes crucial to correlate the PASc samples with either the citrate-bound or -free state of the CitApc. The new assignment performed using ${ }^{1} \mathrm{H}$-detected ssNMR identified some residues of the cytosolic N-terminal helix for the citratebound CitApc, but they were not sufficient for a clear-cut structural conclusion. Two different effects contribute to the chemical shift differences reported in figure 3.17, namely the potential structural rearrangements and the deuterium shift. The latter effect was compensated (see section 3.3.3) to isolate the contributions of the structural differences between the isolated domain and the transmembrane construct.

In general, the absolute values of the chemical shift differences are larger than what observed for the periplasmic domain. In addition, the residues that exhibits a chemical shift difference bigger than $0.5 \mathrm{ppm}$ with the PASc wild-type values, do not cluster in any specific region of the protein and they are found in the Nterminal helix, in the central $\beta$-scaffold and in the major loop (see figure 4.10). This suggests that the influence of the membrane and of the TM helices on the cytosolic domain is not confined to the residues at the membrane-cytosol interface, but instead it propagates to the whole domain. Alanine 216 and serine 304 are the only residues for which the values are significantly closer to the values of PASc wild-type, suggesting that cytosolic domain of CitApc adopts a parallel structure in the citrate-bound state and supporting what proposed in the previous paragraph. Assignments of the N-terminal helix of the cytosolic domain are not yet available for CitApc in the citrate-free state, so it is not possible at the moment to know which one of the PASc conformations best represents the OFF state based on chemical shift analysis.

Amide exchange properties Due to the incomplete assignments of the cytosolic domain in the transmembrane construct, analysing the amide exchange pattern established a convenient method to obtain informations about the OFF and the ON states of the CitApc construct. For the citrate-free CitApc, 5 of the cross-peaks assigned to the cytosolic PAS are retained in the 2D NCA measured in deuterated buffer, while none of the cytosolic residues of citrate-bound CitApc is visible (see figure 4.11). 


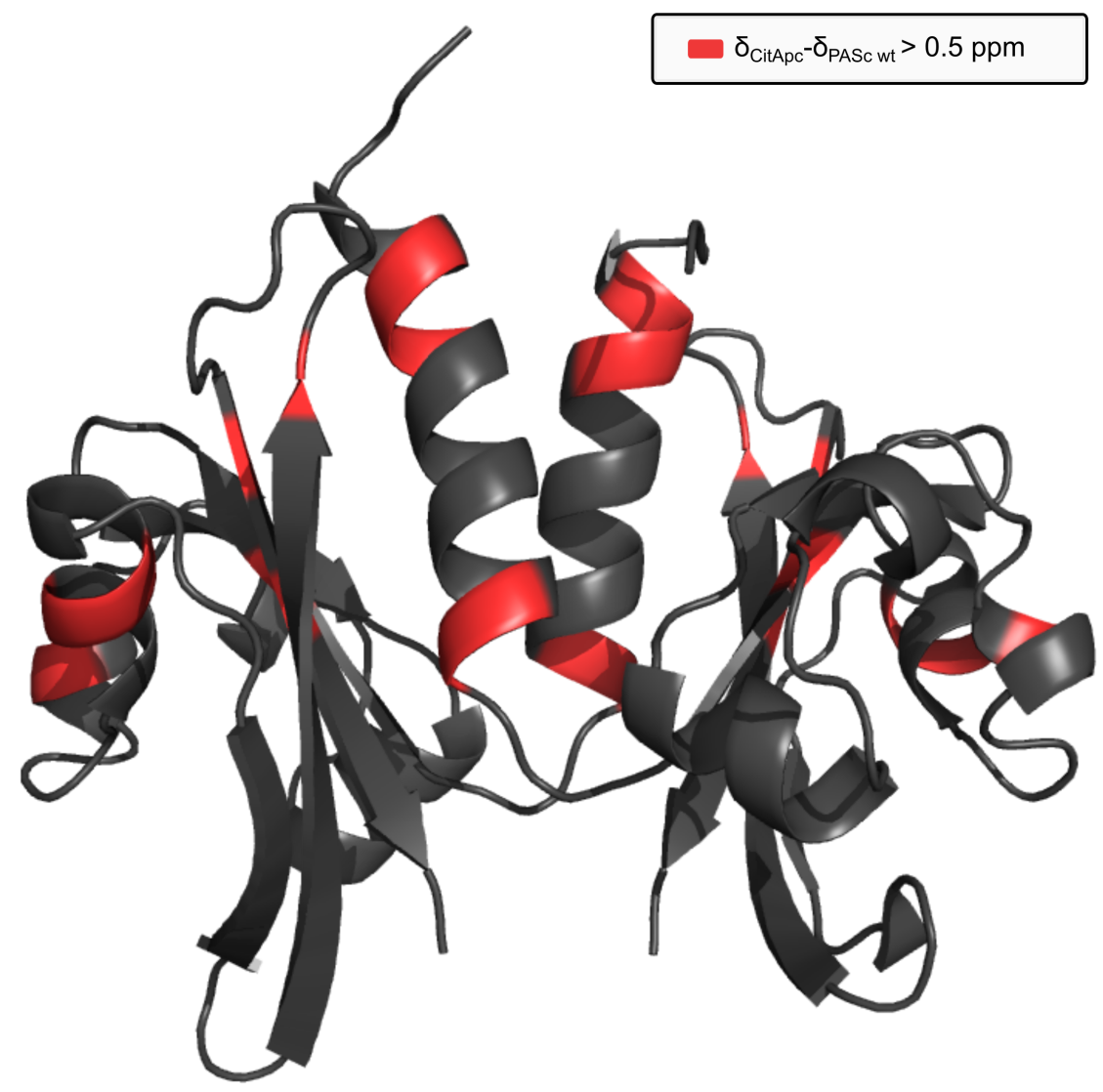

Figure 4.10: The residues that have a chemical shift difference bigger than $0.5 \mathrm{ppm}$ with the PASc wild-type values are reported in red on the PASc wild-type crystal structure.

Thus, the changes induced by the citrate binding are affect also the amide exchange properties. The citrate-free state, which is less prone to amide exchange, is then expected to be more rigid than the bound state, in agreement with the overall destabilization mechanism proposed for G. thermodenitrificans CitA and for E. coli DcuS (Etzkorn et al., 2008; Salvi et al., 2017).

However, comparing the amide exchange properties of the isolated PAS domain with the ones of the transmembrane construct shows once more that structural rearrangements may occur together with the changes in the dynamics. For the PASc wild-type, a total of 19 signals are retained in the ${ }^{15} \mathrm{~N}-\mathrm{HSQC}$ measured in deuterated buffer while, in the same experiment, 35 and 31 peaks are observed for the PASc N288D and R289D mutants, respectively (see figure 3.7). Thus, the amide exchange properties of the citrate-bound state are best represented by the PASc wild-type, while the properties of citrate-free CitApc are more similar to the PASc N288D. This conclusion was derived also based on the overall visibility 


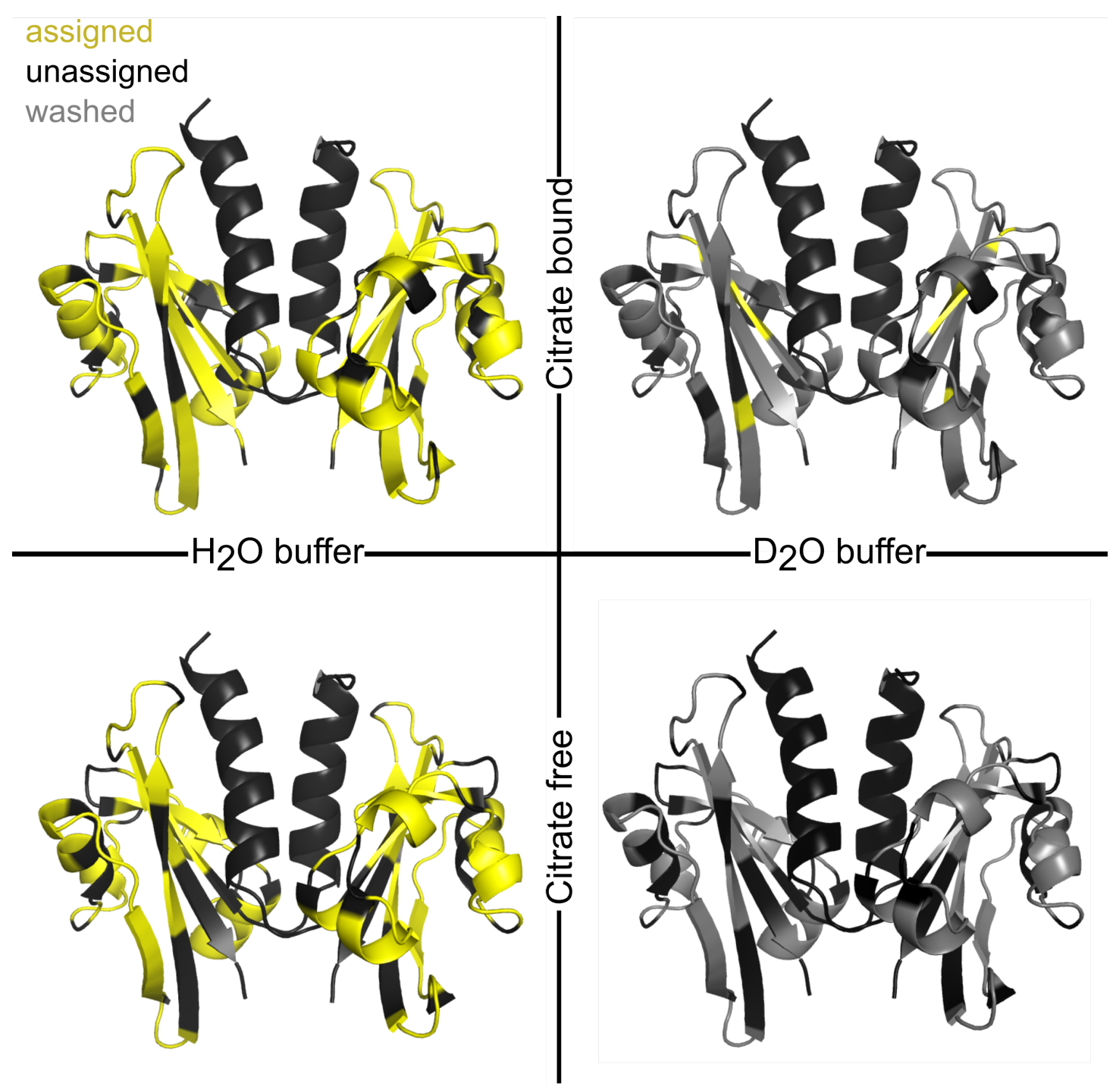

Figure 4.11: Visibility of the cytosolic domain of the CitApc in $\mathrm{H}_{2} \mathrm{O}$ buffer for citrate bound state (top left), in $\mathrm{H}_{2} \mathrm{O}$ buffer for citrate free state (bottom left), in $\mathrm{D}_{2} \mathrm{O}$ buffer for citrate bound state (top right) and in $\mathrm{D}_{2} \mathrm{O}$ buffer for the citrate free state (bottom left). The visible residues are always coloured in yellow, the non-visible always in black. The grey residues on the left side are the one which exchange the amide proton and are not visible in $\mathrm{D}_{2} \mathrm{O}$ buffer. In $\mathrm{D}_{2} \mathrm{O}$ buffer, only some residues in the $\beta 1$ and in the $\beta 4$ retain their visibility in $\mathrm{D}_{2} \mathrm{O}$ buffer for the bound state, while no signal was observed for the free state.

in NMR experiments (see section 4.3.3) and support the hypothesis of a bindinginduced switch from anti-parallel to parallel arrangement.

Additional proofs of the structural rearrangement triggered by the binding event can be deduced looking at the exchange properties and visibility of the last $\beta$ strand of the PASc. The visibility of the last 10 residues is reduced in CitApc by the presence of the citrate (see figure 4.12 right). This difference between the free and the bound state was interpreted exclusively as a modification in the 
PASc dynamics before the alternative structures were found. In the isolated PASc domain, the visibility of the C-terminal $\beta$ strand in deuterated buffer increases in the two mutants compared to the wild-type sample (see figure 4.12 left). Thus, the citra-bound state of PASpc can be associated once more with the isolated PASc wild-type and the parallel structure, while one of two PASc mutants is more similar to citrate-free CitApc (see coloured boxes in figure 4.12).

\begin{tabular}{|c|c|c|c|}
\hline & 300 & 290 & \\
\hline PASc wt & PIKNKQGRVIGAVSTFRNK & PIKNKQGRVIGAVSTFRNK & Citrate bound \\
\hline PASC N288D & PIKNKQGRVIGAVSTFRINK & PIKNKQGRVIGAVSTFRINK & Citrate free \\
\hline PASC R289D & PIKNKQGRVIGAVSTFRINK & & \\
\hline
\end{tabular}

Analogies PASc mutants and citrate free CitApc

Analogies between PASc wt and citrate bound CitApc

Visible in $\mathrm{D}_{2} \mathrm{O}$ buffer and solution NMR

Visible in $\mathrm{H}_{2} \mathrm{O}$ buffer and solid-state NMR

Figure 4.12: Comparison of visibility patterns between the C-terminus of the isolated PASc domain in $\mathrm{D}_{2} \mathrm{O}$ buffer (on the left side) and the C-terminus of the CitApc in $\mathrm{H}_{2} \mathrm{O}$ buffer (on the right side). The last six residues (in the blue boxes) are not visible neither in the PASc wild-type, nor in the CitApc citrate bound. Instead, only the two last residues (green boxes) are invisible for the two PASc mutants and the CitApc citrate free.

As already mentioned, amide exchange patterns do not represent structural features of a protein very faithfully as they are affected by both structural and dynamic features. Therefore it is not possible to discern whether the conclusion just discussed is a consequence of differences in the dimer arrangement or in the dynamics. Nevertheless, the evidence reported so far represent a complete new scenario compared to what was reported previously (Schomburg, 2014). The biological relevance of the alternative crystal structures of CitA PASc was questioned as they were considered not compatible with the functional dimeric state of the full-length receptor. The amide exchange pattern analysis, together with the overall visibility and the chemical shifts comparison, suggests instead that the alternative dimeric conformations may be relevant for the signal relay and the two linkers preceding and following the cytosolic domain in the CitApc are sufficient to allow an alternative dimer conformation in the OFF state.

Solvent PRE Solvent PRE were measured already using solution NMR to investigate the alternative dimeric states of the PASc domain. They are a powerful tool to characterise protein surfaces and inter-molecular complexes and do not depend on secondary structure stability or on the presence of hydrogen bonds, in contrast to amide exchange patterns. To detect eventual rearrangements of the 
cytosolic dimer interface upon the citrate binding event, sPREs of the CitApc were measured for the bound and the free states.

For the citrate-bound sample, the spectral quality is not affected and only the most exposed residues are completely quenched using $5 \mathrm{mM}$ dopant concentration (see picture 3.18). For both periplasmic and cytosolic domain of the CitApc, the sPRE effects are seen at the protein surface, with the least affected residues clustering at the dimer interface or at the core of the protein (see figure 4.13 and 4.14). Notably, the protein surface of the receptor domain is not expected to change drastically, so it has been used as an internal reference for the reliability of the measurement. As shown in figure 4.13, the less affected residues of the periplasmic domain in the bound-state are found in the $\beta$-scaffold and on the N-terminal helix, which forms the dimer interface.

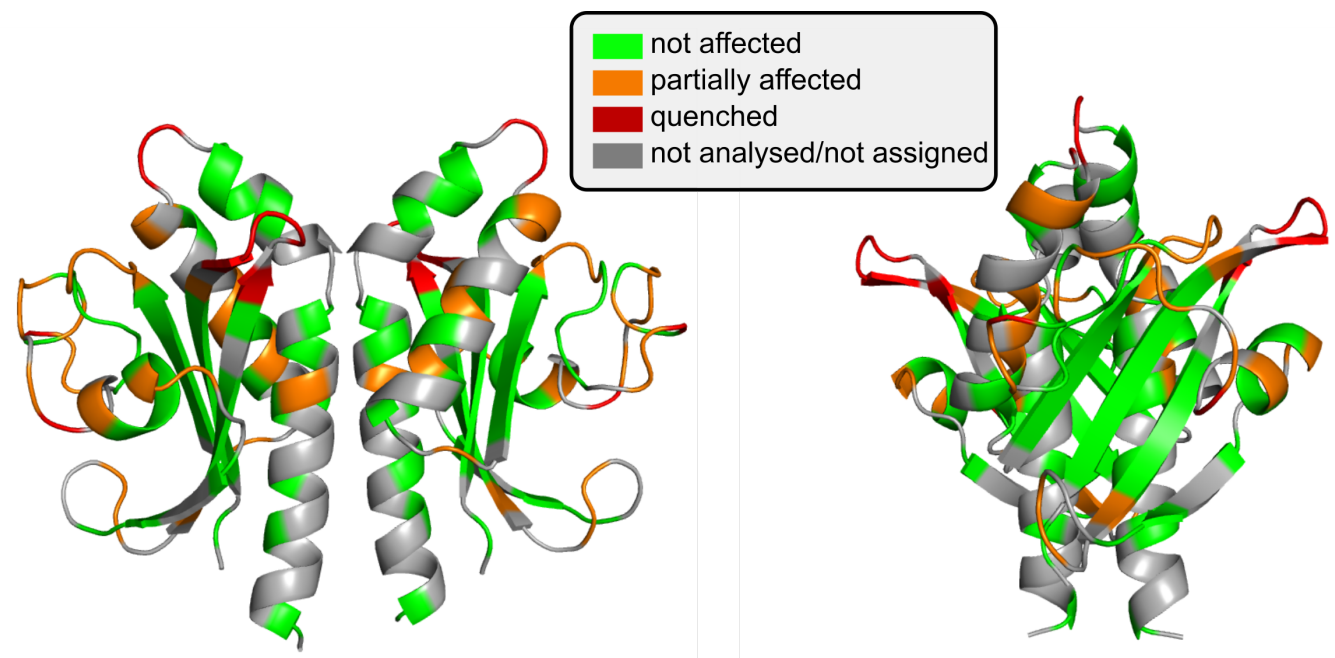

Figure 4.13: Solvent PREs measured on the periplasmic domain of the CitApc in the bound-state. The residues in green are the less affected and located far from the protein surface. The residues in orange or in red are more affected and are found in the proximity if the protein surface.

Even though the sPRE effects for the cytosolic domain were evaluated for a smaller number of peaks, they are numerous enough to analyse the solvent exposed surface (see figure 4.14). As a general trend valid for all the dimer arrangements and already observed for the PAS fold of the periplasmic domain, the major loop is the most exposed element, while the $\beta$-scaffold seems to be the furthest region from the protein surface and is therefore the least affected. Interestingly, the presence of the paramagnetic agent affects the residues on the N-terminal helix in a modest way. The experimental sPREs for the amino acids glutamic acid 202 and glycine 
204 can be explained by all the dimer arrangements, if deviations coming from the presence of the membrane and of the TM domain are taken into account, while the amino acids from leucine 214 to alanine 216 are found far from the surface only if the parallel dimer is adopted in the citrate-bound state. This is in agreement to
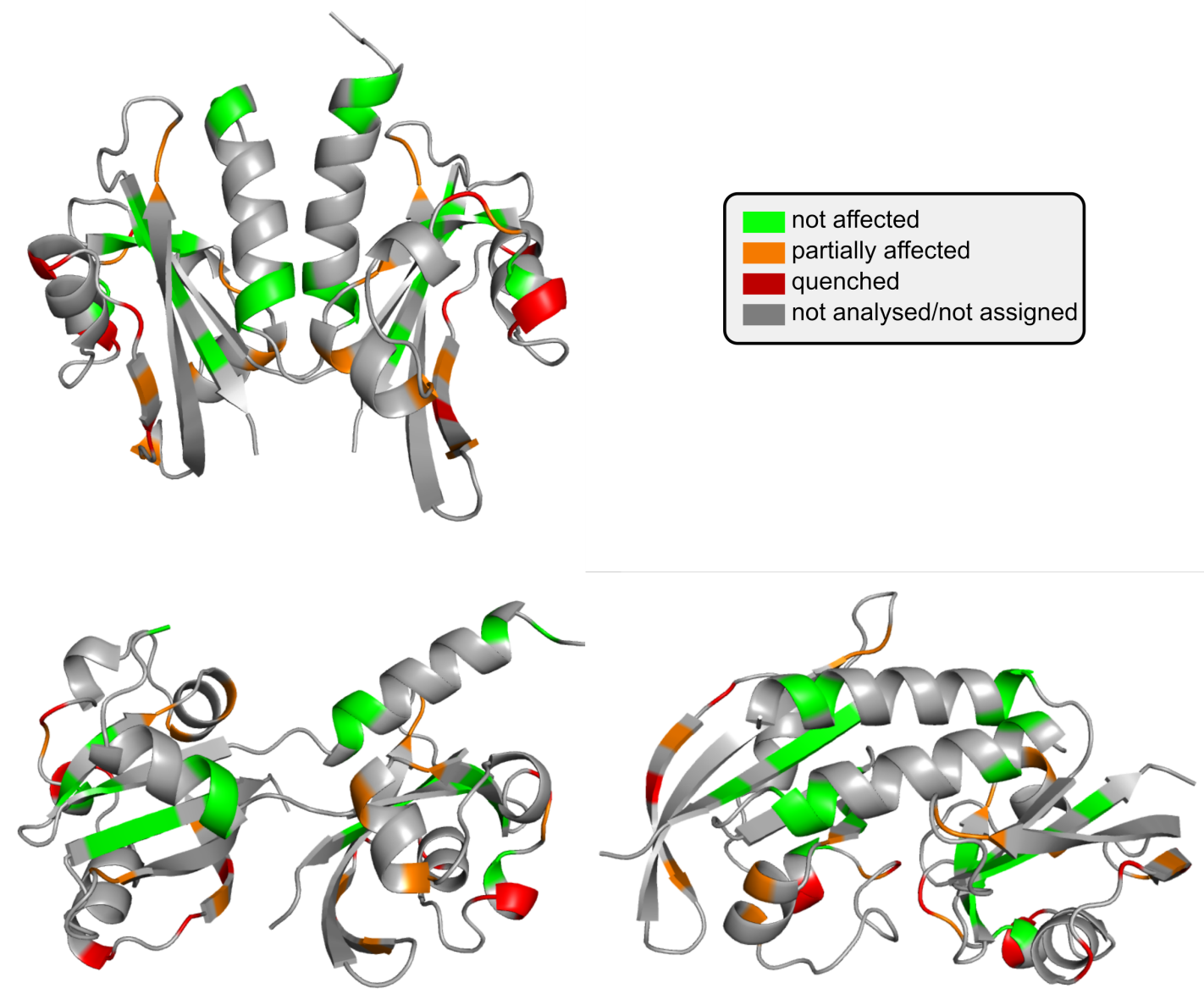

Figure 4.14: Solvent PREs measured on the cytosolic domain of the holo-CitApc. The protein surface is well characterised despite the few residues analysed. The evaluated amino acids in the N-terminal helix are relevant to distinguish which of the possible dimer arrengemnts is adopted in the citrate-bound state.

all the evidences reported in this thesis and suggests once more that the cytosolic domain of the CitApc is adopting the parallel dimer conformation in its active state.

In addition to the protein surface characterization, indirect indication of bulk $\mathrm{T}_{2}$ can be extrapolated from the average values of the intensity ratio between the paramagnetic and the diamagnetic sample. According to the model used (see section 2.4), spurious terms that depend on both $R_{2}$ and on the distance to the 
paramagnetic agent are present if a multidimensional NMR experiment is measured. Therefore, residues with shorter $\mathrm{T}_{2}$ will experience a stronger sPRE effect at the same concentration of paramagnetic agent. Based on this, it can be asserted that the cytosolic domain has a bulk $\mathrm{T}_{2}$ shorter than the periplasmic domain in the citrate-bound state of the CitApc, in agreement with previous studies of HKs, where the cytosolic domain showed faster dynamics compared to the periplasmic domain (Etzkorn et al., 2008; Salvi et al., 2017).

\subsubsection{Compatibility of the antiparallel-to-parallel switch}

Structural information on the signalling mechanism of the cytosolic PAS domain is scarce. The overall destabilization signalling mechanism has been proposed for $E$. coli DcuS PASc relying on ssNMR and mutational studies (Etzkorn et al., 2008; Monzel et al., 2013). The same model was proposed also for G. thermodenitrificans CitA, based on ssNMR studies of the CitApc segment (Salvi et al., 2017). However, as shown for NarQ (Gushchin et al., 2017), signalling mechanisms are not so simple and can be a combination of many motions. Thus, alongside with the overall destabilization of the cytosolic domain, eventual structural rearrangements may happen and be relevant to the signalling relay from the PASc to the DHp domain.

Since the crystal structures of the CitA PASc showing alternative dimer conformations of figure 3.5 were solved, it was speculated that the antiparallel and open arrangements may be relevant for the signalling mechanism in G. thermodenitrificans CitA. Following this idea, the existence of the alternative dimer arrangements was confirmed in the isolated PASc domain. Later, the cytosolic domain of the CitApc was studied to investigate whether the alternative dimeric conformations not only can be adopted in the context of the membrane, but that the rearrangement is triggered by the binding of the citrate in the periplasmic domain. The results just discussed show unambiguously that the bound state of CitApc cytosolic PAS domain adopt the parallel dimer arrangement, as in figure $3.5 \mathrm{~A}$. On the other hand, even if sPREs still have to be measured for the citrate-free CitApc, the anti-parallel dimer (figure $3.5 \mathrm{C}$ ) seems to resemble the conformation of the CitA cytosolic PAS domain in absence of the ligand, based on overall visibility and amide exchange properties (see paragraph 4.3.3). Thus, if supported by sPRE data on the citrate-free CitApc, the antiparallel-to-parallel (see figure 4.15) switch 
would be the relay mechanism from the PASc to the DHp domain, while the open dimer, which could not be associated with the CitApc, either is just adopted in the isolated PASc or represents a low populated, non-detectable intermediate.

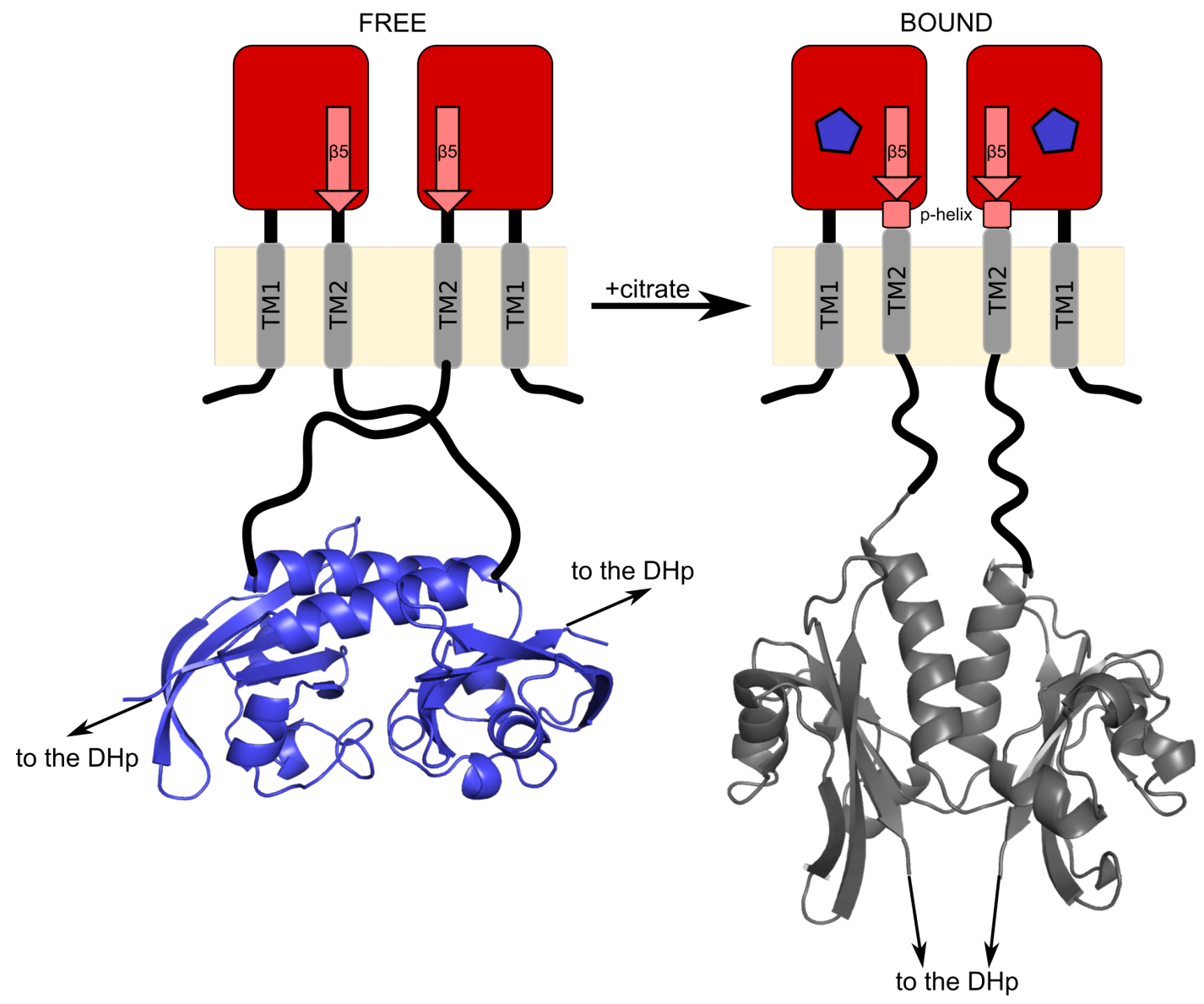

Figure 4.15: The antiparallel-to-parallel switch proposed for the signalling mechanism of $G$. thermodenitrificans CitA PASc.

The suggested mechanism involves certainly some drastic rearrangement of the dimeric conformation and its compatibility with previous findings on HKs needs to be discussed.

The antiparallel-to-parallel switch complements the overall destabilization model proposed previously (Etzkorn et al., 2008; Salvi et al., 2017): based on overall NMR visibility and amide exchange properties, the PASc wild-type, which adopt predominantly the parallel arrangement, is less rigid than the PASc N288D, which is found mainly in the anti-parallel dimeric conformation. Still, the swap from antiparallel to parallel arrangement may result not easy to conceive. First, because conformations alternative to the parallel dimer have never been reported for PAS domains and second, because the inter-subunit distance at the C-termini and at 
the N-termini of the anti-parallel structure raises questions about the compatibility of this arrangement with the functional dimeric state of the full-length CitA, as explained below.

In the anti-parallel structure, the N-terminal helices run almost parallel to the membrane surface, in contrast to what observed in NarQ (Gushchin et al., 2017). Gushchin et al. (2017) reported that the TM2 is connected to the N-terminal helix of the cytosolic HAMP domain without any break in the secondary structure in both holo- and apo-state (Gushchin et al., 2017). This would be possible for the parallel dimer, but unlikely for the anti-parallel. In addition, the $10 \AA$ displacement at the N-termini occurring in the antiparallel-to-parallel switch was never reported and it is in contrast with what reported for E. coli NarQ. It needs to be considered however that a twelve residues linker is located between the end of the TM2 and the first amino acid of the PASc domain, while only five residues are present in NarQ between the end of TM2 and the first amino acid of the HAMP domain. Thus, the longer linker of $G$. thermodenitrificans CitA could permit the antiparallel-to-parallel switch, allowing the PASc domains to adopt an atomic mechanism different from the one observed in NarQ for the HAMP domain.

Also the displacement at the C-termini is much larger in the antiparallel-to-parallel switch than what reported for NarQ. In the latter, a difference of $6 \AA$ is observed at the C-termini between the holo and the apo structure (Gushchin et al., 2017), while the largest motion estimated for CitA using the proposed model is about $20 \AA$. In addition, it can be questioned whether the $6 \AA$ motion observed at the C-termini of the NarQ crystal structure directly triggers for the kinase activity, as more than hundred residues are located between the end of the HAMP domain and the beginning of the kinase core. The displacement at the HAMP domain could then be amplified or further modified before it reaches the kinase core. On the other hand, only 21 residues are located between the end of the PASc and the kinase core in the $G$. thermodenitrificans CitA. The length of the linker guarantees that the output signal of the PASc is relayed directly to the DHp and the kinase core and, at the same time, it could allow the antiparallel-to-parallel switch. 


\subsection{Conclusion and perspective}

In this work, progress on the understanding of the signalling mechanism of Geobacillus thermodenitrificans CitA has been presented. For the periplasmic domain, the shortening of the C-terminal $\beta$-strand, already reported in Salvi et al. (2017), could be extended to the residue aspartate 157 (see figure 4.8). It can be concluded that, in the presence of the ligand, the last residues of the periplasmic domain fold into an $\alpha$-helix which may connect with further helical residues to TM2 uninterruptedly.

A greater effort has been made to understand the role of the cytosolic domain in the signalling mechanism, also combining NMR with other biophysical techniques. In particular, the capability of the $G$. thermodenitrificans PASc to adopt various dimer arrangements has been proven for the isolated domain first and then connected to the citrate binding event in the receptor domain of the transmembrane construct and the antiparallel-to-parallel switch is discussed as a signalling model. In this mechanism, the ON state of the $G$. thermodenitrificans is represented by the PASc wild-type sample and its parallel dimer arrangement (see figure 4.15 on the right), while the PASc domains of CitApc in the OFF state have the dimer arrangement found for the isolated domains in the N288D mutant (see figure 4.15 on the left).

Although NMR data of the transmembrane helices remain scarce and it is still unclear how the transmembrane helices transfer the signal from the periplasm to the cytosol, some progress has been made in this direction thanks to the new ${ }^{1} \mathrm{H}$ detected ssNMR experiments, where the numerous cross-peaks not yet assigned represent a good starting point for the characterization of the TM1 and TM2 helices. If a satisfying assignment of the TM region is reached, different approaches could be exploited to confirm the piston model, which is thought to be the transmission mechanism based upon the pull at the C-terminus of the periplasmic domain and on a previous study of DcuS (Monzel and Unden, 2015). The most general strategy is represented by measuring inter-molecule contacts via NOESY experiments, as also done for the characterization of the isolated PASc dimeric state (see section 3.2). This approach is certainly not trivial in ssNMR, but using MAS speed faster than $100 \mathrm{kHz}$ and selective labelling will guarantee higher resolution, less crowding of the spectra and the possibility to look directly at through-space contacts between the side chains. A second possibility is the use of 
lipids spin-labelled at different positions. This strategy could also detect motions different from the piston-like displacement. Indeed, questions about the contribution of different motions were already raised (Monzel and Unden, 2015) and subsequently observed in NarQ (Gushchin et al., 2017). Ideally, the structural information gained on the CitApc construct needs to be confirmed on the full length CitA. Due to the increased protein size, full-length CitA represents a new challenge for ssNMR. Therefore, it is probably fundamental to tackle this large system with other biophysical techniques, e.g. electron paramagnetic resonance or fluorescence microscopy, that can be used to measure the inter-unit distances of the dimer in the $\mathrm{ON}$ and in the OFF state. In addition, solvent exposure of the linkers is expected to show significant differences between the ON and the OFF state and hydrogen/deuterium exchange mass spectrometry conducted on the full-length protein can be exploited to characterize the changes induced by the citrate binding. If these measurements will confirm what was observed so far for CitApc fragment, a well established mechanism for the signalling relay in the $G$. thermodenitrificans CitA might be elucidated, leading to defined models for the $\mathrm{OFF}$ and the ON state of this protein. 



\section{A. Appendix}

\section{A.1 $\quad{ }^{13} \mathrm{C}-{ }^{15} \mathrm{~N}$ double edited NOESY}

\begin{tabular}{|c|c|c|c|c|c|c|c|c|c|c|c|}
\hline \multicolumn{4}{|c|}{ PASc wild-type } & \multicolumn{4}{|c|}{ PASc R289D } & \multicolumn{4}{|c|}{ PASc N288D } \\
\hline Cross-peak assignment & $\overline{\mathrm{PD}}$ & $\mathrm{AD}$ & OD & Cross-peak assignment & $\mathrm{PD}$ & $\mathrm{AD}$ & $\overline{\mathrm{OD}}$ & Cross-peak assignment & $\mathrm{PD}$ & $\mathrm{AD}$ & OD \\
\hline $202 \mathrm{E} \mathrm{HN}-203 \mathrm{I} \mathrm{H} \delta$ & 10.70 & 19.82 & 37.22 & 202E HN-213I H $\gamma 1$ & 17.97 & 6.61 & 38.51 & 203I HN-203I H $\delta$ & 6.88 & 18.66 & 35.27 \\
\hline 203I HN-203I H $\beta$ & 9.08 & 20.36 & 36.54 & 203I HN-203I H $\beta$ & 9.08 & 20.36 & 36.54 & 203I HN-203I H $\gamma 2$ & 6.59 & 19.37 & 35.84 \\
\hline 203I HN-203I H $\delta$ & 6.88 & 18.66 & 35.27 & 203I HN-203I H $\delta$ & 6.88 & 18.66 & 35.27 & 203I HN-203I H $\gamma 1$ & 7.69 & 20.33 & 36.43 \\
\hline 203I HN-203I H $\gamma 1$ & 7.69 & 20.33 & 36.43 & 203I HN-203I H $\gamma 2$ & 6.59 & 19.37 & 35.84 & 203I HN-203I H $\beta$ & 9.08 & 20.36 & 36.54 \\
\hline 203I HN-203I H $\gamma 2$ & 6.59 & 19.37 & 35.84 & $204 \mathrm{G} \mathrm{HN}-203 \mathrm{I} \mathrm{H} \beta$ & 10.19 & 20.10 & 34.32 & 204G HN-290I H $\gamma 2$ & 16.07 & 5.90 & 21.99 \\
\hline 203I HN-214L H $\delta$ & 18.34 & 6.78 & 34.53 & $204 \mathrm{G}$ HN-203I H $\delta$ & 7.17 & 17.00 & 35.90 & $208 \mathrm{~N} \mathrm{HN}-292 \mathrm{I} \mathrm{H} \gamma 2$ & 5.23 & 3.59 & 11.47 \\
\hline 293K HN-216A H $\beta$ & 11.41 & 18.04 & 5.51 & 204G HN-203I H $\gamma 1$ & 7.68 & 18.99 & 31.44 & 212A HN-300I H $\gamma 2$ & 11.40 & 7.34 & 8.19 \\
\hline & & & & 204G HN-295K H $\gamma$ & 6.91 & 12.14 & 24.89 & $212 \mathrm{~A} H \mathrm{HN}-300 \mathrm{I} \mathrm{H} \delta$ & 8.23 & 5.79 & 4.76 \\
\hline & & & & $204 \mathrm{G} \mathrm{HN}-303 \mathrm{~V} \mathrm{H} \gamma 1$ & 15.80 & 3.54 & 16.19 & 212A HN-300I H $\gamma 1$ & 8.90 & 8.05 & 6.80 \\
\hline & & & & 212A HN-292I H $\delta$ & 5.75 & 10.02 & 4.98 & 213I HN-300I H $\gamma 2$ & 13.34 & 9.92 & 9.16 \\
\hline & & & & 212A HN-292I H $\gamma 2$ & 4.71 & 9.16 & 6.63 & 213I HN-300I H $\delta$ & 10.10 & 8.43 & 5.34 \\
\hline & & & & 213I HN-292I H $\delta$ & 6.05 & 12.27 & 3.64 & 213I HN-303V H $\gamma 2$ & 4.12 & 15.19 & 6.25 \\
\hline & & & & 213I HN-292I H $\gamma 1$ & 5.40 & 13.53 & 5.77 & 214L HN-213I H $\delta$ & 4.47 & 14.49 & 23.09 \\
\hline & & & & 213I HN-303V H $\gamma 2$ & 4.12 & 15.19 & 6.25 & 214L HN-213I H $\gamma 2$ & 4.81 & 15.06 & 20.01 \\
\hline & & & & 216A HN-292I H $\delta$ & 10.15 & 15.46 & 6.20 & $216 \mathrm{~A} H \mathrm{HN}-303 \mathrm{~V} \mathrm{H} \alpha$ & 8.54 & 20.53 & 8.77 \\
\hline & & & & 216A HN-303V H $\gamma 1$ & 4.78 & 18.79 & 4.64 & 216A HN-292I H $\gamma 2$ & 9.54 & 13.98 & 4.72 \\
\hline & & & & $222 \mathrm{~V}$ HN-213I H $\delta$ & 7.88 & 19.92 & 7.01 & 216A HN-303V H $\gamma 1$ & 4.78 & 18.79 & 4.65 \\
\hline & & & & $222 \mathrm{~V}$ HN-213I H $\gamma 2$ & 6.32 & 20.77 & 6.49 & $216 \mathrm{~A}$ HN-303V H $\gamma 2$ & 5.77 & 19.16 & 6.49 \\
\hline & & & & $293 \mathrm{~K} \mathrm{HN}-212 \mathrm{~A} \mathrm{H} \beta$ & 5.03 & 10.38 & 4.88 & 216A HN-303V H $\beta$ & 7.14 & 21.38 & 6.48 \\
\hline & & & & $293 \mathrm{~K} \mathrm{HN}-216 \mathrm{~A} \mathrm{H} \beta$ & 11.41 & 16.30 & 5.51 & 217I HN-221I H $\gamma 2$ & 7.72 & 21.76 & 6.42 \\
\hline & & & & & & & & 217I HN-221I H $\delta$ & 4.86 & 23.80 & 6.17 \\
\hline & & & & & & & & 217I HN-303V H $\gamma 2$ & 6.12 & 21.11 & 5.32 \\
\hline & & & & & & & & 217I HN-305T H $\gamma 2$ & 3.52 & 27.86 & 5.72 \\
\hline & & & & & & & & $219 \mathrm{E} \mathrm{HN}-290 \mathrm{I} \mathrm{H} \delta$ & 10.88 & 28.20 & 9.71 \\
\hline & & & & & & & & $219 \mathrm{E} \mathrm{HN}-290 \mathrm{I} \mathrm{H} \gamma 2$ & 11.77 & 31.93 & 9.50 \\
\hline & & & & & & & & $293 \mathrm{~K} H \mathrm{~N}-212 \mathrm{~A} \mathrm{H} \beta$ & 5.03 & 10.38 & 4.88 \\
\hline & & & & & & & & $293 \mathrm{~K} H \mathrm{~N}-208 \mathrm{~N} \mathrm{H} \alpha$ & 5.69 & 6.21 & 11.42 \\
\hline & & & & & & & & $293 \mathrm{~K} H \mathrm{~N}-216 \mathrm{~A} \mathrm{H} \beta$ & 9.87 & 16.30 & 5.51 \\
\hline & & & & & & & & $294 \mathrm{~N} \mathrm{HN}-212 \mathrm{~A} \mathrm{H} \beta$ & 9.23 & 8.67 & 7.34 \\
\hline
\end{tabular}

Table A.1: NOE contacts in the ${ }^{13} \mathrm{C}^{15}{ }^{15}$ double edited NOESY and distances from the crystal structure. The NOE contacts assigned in the ${ }^{13} \mathrm{C}-{ }^{15} \mathrm{~N}$ double edited NOESY spectra of the CitA PASc wild type and N288D and R289D mutants, together with the distances in $\AA$ expected from the crystal structure of each of the dimer arrangement (PD:Parallel Dimer, AD:Anti-parallel Dimer, OD:Open Dimer). All the cross-peaks are assumed to be inter-unit as a consequence of the labelling scheme and NMR pulse sequence.

\section{A.2 NMR assignments}


Table A.2: Chemical shift assignment of isolated PASc wild-type. All the values are reported in ppm.

PASc wild-type chemical shifts $[\mathrm{ppm}]$

\begin{tabular}{|c|c|c|c|c|c|c|c|c|c|c|c|c|c|c|c|}
\hline & $\mathrm{H}$ & $\mathrm{N}$ & $\mathrm{ND}$ & $\mathrm{NE}$ & HA & HB & $\mathrm{HG}$ & $\mathrm{HD}$ & $\mathrm{HE}$ & $\mathrm{C}$ & $\mathrm{CA}$ & $\mathrm{CB}$ & $\mathrm{CG}$ & $\mathrm{CD}$ & $\mathrm{CE}$ \\
\hline 200 Pro & - & - & - & - & 4.3 & $2.27,1.86$ & $2.04,1.96$ & $3.82,3.73$ & - & 177.87 & 64.43 & 31.84 & 27.66 & 50.68 & - \\
\hline $201 \mathrm{Glu}$ & 8.45 & 119.26 & - & - & 4.1 & 1.94 & 2.23 & - & - & 177.43 & 57.91 & 29.64 & 36.6 & - & - \\
\hline $202 \mathrm{Glu}$ & 8.04 & 121.23 & - & - & 4.08 & 1.95 & $2.23,2.20$ & - & - & 177.53 & 57.83 & 29.71 & 36.59 & - & - \\
\hline 203 Ile & 7.99 & 120.6 & - & - & 3.86 & 1.8 & $1.10,0.79,1.38$ & 0.71 & - & 177.06 & 62.76 & 38.03 & $28.09,17.63$ & 12.8 & - \\
\hline 204 Gly & 8.24 & 109.52 & - & - & $3.86,3.88$ & - & - & - & - & - & 46.4 & - & - & - & - \\
\hline 205 Leu & - & - & - & - & 4.18 & 1.54 & - & - & - & - & 56.88 & 42.07 & 26.92 & $23.61,24.67$ & - \\
\hline 206 Leu & 8.1 & 118.18 & - & - & 4.14 & 1.44 & - & 0.83 & - & - & 56.94 & 41.9 & 27.06 & $23.38,25.03$ & - \\
\hline 207 Tyr & - & - & - & - & 4.13 & $3.03,3.03$ & - & - & - & - & 61.47 & 38.22 & - & - & - \\
\hline 208 Gln & - & - & - & - & 4.17 & - & 1.96 & - & - & - & 57.2 & 29.95 & 36.28 & - & - \\
\hline $209 \mathrm{Glu}$ & 8.03 & 116.95 & - & - & 3.84 & 1.87 & $2.16,2.14$ & - & - & - & 58.62 & 28.38 & 33.33 & - & - \\
\hline 210 Lys & - & - & - & - & 3.74 & 1.7 & - & - & 2.86 & - & 59.46 & 31.97 & 25.7 & - & 42.29 \\
\hline $211 \mathrm{Gln}$ & - & - & - & - & 3.83 & 2.13 & 2 & - & - & - & 59.51 & 30.12 & 36.79 & - & - \\
\hline 212 Ala & 7.65 & 121.56 & - & - & 4.13 & 1.33 & - & - & - & - & 53.48 & 20.31 & - & - & - \\
\hline 213 Ile & - & - & - & - & - & 1.83 & 1.01 & 0.66 & - & - & 62.82 & 39.42 & $27.25,17.44$ & 14.15 & - \\
\hline 214 Leu & 8.7 & 122.88 & - & - & 3.78 & $1.40,1.89$ & 0.7 & $0.71,0.78$ & - & - & 57.8 & 41.7 & 27.45 & $24.08,25.33$ & - \\
\hline $215 \mathrm{Glu}$ & 7.76 & 114.61 & - & - & 4.28 & 2.06 & $2.40,2.33$ & - & - & - & 57.81 & 29.87 & 36.15 & - & - \\
\hline 216 Ala & 7.64 & 120.41 & - & - & 4.03 & 1.33 & - & - & - & - & 54.97 & 18.7 & - & - & - \\
\hline 217 Ile & 7.72 & 117.5 & - & - & 3.36 & 1.77 & $0.67,1.75,0.67$ & 0.6 & - & - & 65.91 & 38.18 & $29.48,17.64$ & 14.08 & - \\
\hline 218 Arg & - & - & - & 114.21 & 4.58 & $1.05,2.29$ & 1.6 & $2.88,3.42$ & 8.79 & - & 55.92 & 30.76 & 28.02 & 42.59 & - \\
\hline 219 Glu & - & - & - & - & 3.9 & $2.10,2.20$ & $2.44,2.44$ & - & - & - & 59.85 & 28.14 & 34.16 & - & - \\
\hline
\end{tabular}




\begin{tabular}{|c|c|c|c|c|c|c|c|c|c|c|c|c|c|c|c|}
\hline 220 Gly & 8.9 & 106.95 & - & - & $2.82,4.39$ & - & - & - & - & 172.04 & 45.36 & - & - & - & - \\
\hline 221 Ile & 7.91 & 122.09 & - & - & 4.87 & 1.14 & $0.54,1.43$ & 0.64 & - & 173.83 & 60.07 & 42.24 & $27.43,17.03$ & 14.38 & - \\
\hline $222 \mathrm{Val}$ & 8.3 & 123.01 & - & - & 4.71 & 1.84 & $0.81,0.94$ & - & - & 172.89 & 60.85 & 36.02 & $21.46,22.18$ & - & - \\
\hline 223 Ala & 8.67 & 127.09 & - & - & 5.5 & 1.21 & - & - & - & 176.52 & 49.85 & 23.53 & - & - & - \\
\hline 224 Ile & 8.71 & 110.71 & - & - & 5.51 & 1.67 & $0.61,1.37$ & 0.61 & - & 176.82 & 58.37 & 42.89 & $24.88,18.05$ & 15.24 & - \\
\hline 225 Asn & 7.89 & 119.09 & 110.89 & - & 5.07 & $2.89,3.57$ & - & $6.92,7.63$ & - & 177 & 50.91 & 38.79 & 176.51 & - & - \\
\hline 226 Gln & 7.91 & 115.67 & - & 111.09 & 3.77 & $1.99,1.94$ & $2.25,2.30$ & - & $7.55,6.72$ & 176.49 & 58.72 & 28.12 & 33.6 & 179.74 & - \\
\hline 227 Glu & 7.57 & 116.88 & - & - & 4.23 & $1.96,2.10$ & $2.19,2.15$ & - & - & 176.95 & 56.27 & 29.46 & 36.61 & - & - \\
\hline 228 Gly & 8 & 106.78 & - & - & $3.48,4.25$ & - & - & - & - & 177.62 & 45.27 & - & - & - & - \\
\hline $229 \mathrm{Thr}$ & 7.85 & 117.65 & - & - & 4.18 & 3.71 & 0.88 & - & - & 174.23 & 61.61 & 69.06 & 22.68 & - & - \\
\hline 230 Ile & 8.24 & 123.78 & - & - & 4.41 & 1.92 & $0.74,0.82,1.79$ & 0.7 & - & 177.5 & 63.05 & 38.16 & $17.98,29.26$ & 14.6 & - \\
\hline $231 \mathrm{Thr}$ & 9.18 & 120.65 & - & - & 4.31 & 4.15 & 1.08 & - & - & 175.41 & 61.5 & 69.62 & 22.74 & - & - \\
\hline 232 Met & 7.26 & 121.58 & - & - & 4.45 & $2.29,2.33$ & $1.96,1.68$ & - & - & 173.08 & 56.37 & 32.29 & 36.22 & - & - \\
\hline $233 \mathrm{Val}$ & 8.49 & 121.44 & - & - & 5.16 & 2.03 & $0.85,0.97$ & - & - & 175.46 & 60.34 & 35.08 & $21.07,20.95$ & - & - \\
\hline 234 Asn & 7.81 & 121.72 & 114.06 & - & 5.13 & $3.68,2.50$ & - & $7.57,8.30$ & - & - & 50.27 & 40.73 & - & - & - \\
\hline 235 Gln & 8.84 & 119.32 & - & - & 4.09 & - & - & - & - & - & 59.73 & - & 36.62 & - & - \\
\hline $236 \mathrm{Thr}$ & - & - & - & - & 3.82 & 3.82 & 1.14 & - & - & 176.29 & 66.72 & 68.77 & 21.99 & - & - \\
\hline 237 Ala & 7.62 & 121.83 & - & - & 4 & 1.48 & - & - & - & 178.4 & 55.72 & 19.05 & - & - & - \\
\hline 238 Leu & 8.22 & 117.44 & - & - & 3.81 & $1.89,1.60$ & 0.63 & 0.59 & - & 178.72 & 58.79 & 40.62 & 25.94 & 24.15 & - \\
\hline 239 Lys & 7.89 & 117.98 & - & - & 4.08 & $1.91,1.91$ & $1.51,1.42$ & $1.64,1.64$ & 2.92 & 180.46 & 59.13 & 32.01 & 25 & 29.04 & 42.13 \\
\hline 240 Leu & 7.69 & 120.28 & - & - & 4.02 & $1.79,1.25$ & 1.67 & $0.70,0.71$ & - & 178.29 & 57.64 & 42.21 & 27.01 & $25.28,23.35$ & - \\
\hline 241 Leu & 7.89 & 115.4 & - & - & 4.11 & $1.64,1.93$ & 1.79 & $0.72,0.76$ & - & 176.83 & 55.44 & 43.05 & 26.91 & $25.05,23.08$ & - \\
\hline 242 Gly & 7.8 & 106.55 & - & - & $3.92,3.67$ & - & - & - & - & 174.19 & 45.73 & - & - & - & - \\
\hline $243 \mathrm{Tyr}$ & 8 & 119.01 & - & - & 4.62 & $2.41,3.01$ & - & - & - & 174.87 & 57.44 & 42.44 & - & - & - \\
\hline 244 Asp & 8.43 & 117.74 & - & - & 4.55 & $2.55,2.73$ & - & - & - & 176.07 & 54.23 & 41.98 & - & - & - \\
\hline 245 Asn & 7.66 & 115.6 & 113.76 & - & 4.72 & $2.72,2.75$ & - & $6.83,7.74$ & - & 174.92 & 52.92 & 41.08 & 176.59 & - & - \\
\hline 246 Glu & 9.39 & 123.48 & - & - & 3.88 & $2.00,1.94$ & $2.20,2.14$ & - & - & 177.5 & 58.38 & 29.09 & 35.48 & - & - \\
\hline
\end{tabular}




\begin{tabular}{|c|c|c|c|c|c|c|c|c|c|c|c|c|c|c|c|}
\hline 247 Arg & 8.58 & 121.14 & - & - & 3.99 & $1.76,1.79$ & $1.57,1.56$ & $3.10,3.10$ & - & 176.76 & 58.44 & 29.5 & 27.16 & 43.15 & - \\
\hline 248 Asn & 7.97 & 115 & 111.69 & - & 4.68 & $2.62,2.90$ & - & $7.66,6.98$ & - & 174.12 & 54.01 & 39.18 & 176.71 & - & - \\
\hline $249 \mathrm{Val}$ & 7.49 & 115.2 & - & - & 3.86 & 1.62 & $0.48,0.34$ & - & - & 176.08 & 62.69 & 33.95 & $21.21,21.86$ & - & - \\
\hline 250 Leu & 7.77 & 120.7 & - & - & 3.67 & $1.58,1.31$ & 1.59 & $0.65,0.77$ & - & 177.97 & 56.72 & 41.36 & 26.72 & $23.10,25.25$ & - \\
\hline 251 Gly & 8.86 & 109.79 & - & - & $3.44,4.25$ & - & - & - & - & 173.99 & 45.19 & - & - & - & - \\
\hline $252 \mathrm{Thr}$ & 7.71 & 117.78 & - & - & 4.6 & 4.1 & 1.16 & - & - & - & 60.79 & 70.13 & 21.2 & - & - \\
\hline 253 Pro & - & - & - & - & 4.53 & $1.77,2.44$ & $2.04,1.88$ & $3.51,3.96$ & - & 179.45 & 62.95 & 32.6 & 28.14 & 51.81 & - \\
\hline 254 Ile & 8.84 & 126.24 & - & - & 3.98 & 1.78 & $1.32,0.98,1.34$ & 0.87 & - & 175.85 & 63.22 & 37.59 & $18.17,30.07$ & 15.12 & - \\
\hline 255 Leu & 8.24 & 118.99 & - & - & 4.17 & $1.64,1.47$ & 1.66 & $0.89,0.88$ & - & 178.33 & 56.46 & 40.49 & 27.01 & $25.35,22.94$ & - \\
\hline 256 Gln & 7.42 & 115.03 & - & 111.42 & 4.05 & $2.01,2.08$ & $2.33,2.31$ & - & $6.83,7.51$ & 176.41 & 57.55 & 28.86 & 34.2 & 179.57 & - \\
\hline 257 Leu & 7.01 & 117.66 & - & - & 4.44 & $1.57,1.64$ & 1.6 & $0.79,0.77$ & - & 176.84 & 55.85 & 44.06 & 27.2 & $25.59,24.20$ & - \\
\hline 258 Ile & 8.53 & 117.25 & - & - & 4.28 & 1.79 & $1.35,0.82,0.42$ & 0.78 & - & - & 58.56 & 38.38 & $26.06,17.93$ & 13.53 & - \\
\hline 259 Pro & - & - & - & - & 4.57 & $2.33,1.76$ & $1.96,1.93$ & $3.29,3.70$ & - & 177.21 & 65.17 & 31.87 & 27.23 & 50.14 & - \\
\hline 260 His & 8.65 & 112.5 & - & - & 4.7 & $3.18,3.02$ & - & - & - & 175.08 & 54.48 & 28.7 & - & - & - \\
\hline 261 Ser & 7.66 & 114.9 & - & - & 4.2 & $3.50,4.02$ & - & - & - & - & 58.99 & 64.48 & - & - & - \\
\hline $262 \mathrm{Arg}$ & - & - & - & 115.3 & - & 1.72 & $1.59,1.58$ & 2.98 & 7.03 & - & 57.06 & 30.4 & 27.01 & 43.11 & - \\
\hline 263 Leu & 8.69 & - & - & - & 3.96 & $1.69,1.25$ & 1.57 & $0.65,0.76$ & - & - & 59.44 & 39.76 & 26.91 & $24.92,23.95$ & - \\
\hline 264 Pro & - & - & - & - & 3.96 & $1.81,2.27$ & $1.92,1.52$ & 3.42 & - & 178.8 & 65.85 & 30.78 & 29.02 & 49.78 & - \\
\hline 265 Glu & 7.21 & 117.96 & - & - & 4.19 & 2.06 & 2.23 & - & - & 178.2 & 58.34 & 28.8 & 35.23 & - & - \\
\hline $266 \mathrm{Val}$ & 7.53 & 120.55 & - & - & 3.79 & 2.03 & $0.52,0.81$ & - & - & 178.5 & 65.34 & 30.93 & $22.40,21.23$ & - & - \\
\hline 267 Ile & 7.17 & 117.62 & - & - & 3.6 & 1.98 & $0.77,1.36,1.04$ & 0.43 & - & 176.88 & 63.18 & 36.23 & $28.05,17.13$ & 12.1 & - \\
\hline $268 \mathrm{Arg}$ & 7.18 & 117.71 & - & 114.35 & 3.97 & $1.92,1.86$ & $1.63,1.45$ & $3.23,3.07$ & 7.69 & 178.79 & 59.17 & 30.98 & 27.69 & 43.15 & - \\
\hline $269 \mathrm{Thr}$ & 8.55 & 107.22 & - & - & 4.17 & 4.19 & 1.25 & - & - & 177.38 & 62.58 & 70.6 & 21.28 & - & - \\
\hline 270 Gly & 8.46 & 111.96 & - & - & $4.00,3.45$ & - & - & - & - & 173.02 & 46.53 & - & - & - & - \\
\hline 271 Gln & 7.98 & 119.24 & - & 112.93 & 4.47 & $1.77,1.94$ & $2.25,2.25$ & - & $7.54,6.84$ & 174.16 & 54.01 & 30.02 & 33.31 & 180.5 & - \\
\hline 272 Ala & 8.3 & 125.42 & - & - & 4.35 & 1.19 & - & - & - & 176.67 & 52 & 20.7 & - & - & - \\
\hline 273 Glu & 8.63 & 119.27 & - & - & 4.36 & $1.82,1.57$ & $2.13,2.08$ & - & - & 174.05 & 54.94 & 32.39 & 35.56 & - & - \\
\hline
\end{tabular}




\begin{tabular}{|c|c|c|c|c|c|c|c|c|c|c|c|c|c|c|c|c|}
\hline 274 Tyr & 8.49 & 120.07 & - & - & 5.22 & $2.92,2.59$ & - & - & - & - & 56.14 & 41.43 & - & - & - & $P$ \\
\hline 275 Asp & 8.83 & - & - & - & 3.97 & $2.67,2.71$ & - & - & - & 174.54 & 55.58 & 39.34 & - & - & - & 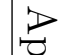 \\
\hline 276 Asp & 8.98 & 120.72 & - & - & 4.88 & $2.63,2.35$ & - & - & - & 175.73 & 53.44 & 42.75 & - & - & - & \\
\hline $277 \mathrm{Glu}$ & 8.34 & 127 & - & - & 4.88 & $1.86,1.87$ & $1.95,2.13$ & - & - & 175.75 & 56.72 & 30.59 & 37.31 & - & - & \\
\hline $278 \mathrm{Met}$ & 9.17 & 124.31 & - & - & 4.7 & $1.83,1.82$ & $2.06,2.29$ & - & - & 172.81 & 54.54 & 36.84 & 30.75 & - & - & \\
\hline $279 \mathrm{Val}$ & 8.29 & 121.43 & - & - & 4.69 & 1.69 & $0.76,0.63$ & - & - & 175.72 & 61.51 & 32.45 & $21.18,20.97$ & - & - & \\
\hline 280 Leu & 8.9 & 129.45 & - & - & 4.54 & $1.04,1.25$ & 1.07 & $0.28,0.30$ & - & 176.09 & 53.93 & 45.38 & 26.86 & $24.82,24.86$ & - & \\
\hline $281 \mathrm{Gly}$ & 9.04 & 114.91 & - & - & $3.95,3.72$ & - & - & - & - & 175.56 & 47.21 & - & - & - & - & \\
\hline 282 Gly & 8.21 & 106.81 & - & - & $3.99,3.62$ & - & - & - & - & 173.54 & 45.01 & - & - & - & - & \\
\hline $283 \mathrm{Glu}$ & 7.82 & 120.91 & - & - & 4.61 & $1.95,1.82$ & $2.09,2.16$ & - & - & 174.86 & 54.31 & 31.91 & 35.38 & - & - & \\
\hline $284 \mathrm{Thr}$ & 8.68 & 118.78 & - & - & 4.68 & 3.93 & 1.06 & - & - & 174.29 & 63.25 & 68.69 & 22.28 & - & - & \\
\hline $285 \mathrm{Val}$ & 9.27 & 121.37 & - & - & 4.98 & 2.01 & $0.68,0.57$ & - & - & 173.32 & 58.39 & 35.49 & $21.17,19.28$ & - & - & \\
\hline 286 Ile & 8.99 & 119.5 & - & - & 4.52 & 1.61 & $1.05,0.71,1.34$ & 0.71 & - & 175.78 & 59.64 & 38.36 & $27.11,18.09$ & 12 & - & \\
\hline 287 Ala & 9.44 & 132.28 & - & - & 5.7 & 1.29 & - & - & - & - & 50.42 & 22.84 & - & - & - & \\
\hline 288 Asn & 8.24 & - & - & - & 5.35 & $2.95,2.30$ & - & - & - & - & 51.95 & 41.71 & - & - & - & \\
\hline $289 \operatorname{Arg}$ & 9.18 & 119.66 & - & - & 5.35 & $1.29,1.61$ & $1.35,1.59$ & - & - & - & 54.28 & 33.82 & 27.24 & - & - & \\
\hline 290 Ile & 9.15 & 122.77 & - & - & 4.63 & 1.75 & $1.28,1.16,1.02$ & 0.74 & - & - & 56.58 & 41.74 & $27.39,17.07$ & 12.63 & - & \\
\hline 291 Pro & - & - & - & - & 4.43 & $1.89,1.64$ & $1.67,1.94$ & $3.18,3.83$ & - & 174.45 & 62.19 & 32.43 & 25.97 & 50.27 & - & \\
\hline 292 Ile & 8.21 & 121.7 & - & - & 4.05 & 1.06 & $0.58,0.59,1.26$ & 0.57 & - & 174.97 & 60.38 & 39.22 & $27.05,18.08$ & 13.73 & - & \\
\hline 293 Lys & 8.56 & 125.83 & - & - & 5.35 & $1.54,1.54$ & $1.02,1.09$ & $1.54,1.54$ & $2.75,2.75$ & 176.58 & 54.17 & 36.4 & 26.18 & 29.58 & 42.22 & \\
\hline 294 Asn & 8.38 & 117.72 & 110.98 & - & 4.69 & $2.75,3.65$ & - & $7.76,6.84$ & - & 177.84 & 50.51 & 38.75 & 176.41 & - & - & \\
\hline 295 Lys & 8.51 & 118.67 & - & - & 3.99 & 1.79 & $1.42,1.47$ & 1.51 & 2.94 & 177.49 & 58.88 & 31.88 & 25.09 & 29.05 & 42.05 & \\
\hline 296 Gln & 7.43 & 115.83 & - & 112.08 & 4.29 & $2.21,2.21$ & $2.26,2.41$ & - & $7.50,6.78$ & 176.2 & 56.42 & 28.43 & 34.81 & 180.69 & - & \\
\hline 297 Gly & 8.05 & 107.48 & - & - & $3.54,4.22$ & - & - & - & - & 173.55 & 45.33 & - & - & - & - & \\
\hline $298 \operatorname{Arg}$ & 7.76 & 121.48 & - & - & 4.25 & $1.59,1.65$ & $1.58,1.42$ & $3.08,3.10$ & - & 175.82 & 55.03 & 30.8 & 27.31 & 43.21 & - & \\
\hline $299 \mathrm{Val}$ & 8.75 & 124.86 & - & - & 4.17 & 2.13 & $0.98,1.13$ & - & - & 176.54 & 63.71 & 31.08 & $23.75,22.66$ & - & - & \\
\hline 300 Ile & 8.69 & 120.03 & - & - & 4.55 & 2.16 & $0.78,0.90,0.72$ & 0.7 & - & 175.26 & 60.61 & 39.03 & $18.31,26.45$ & 14.05 & - & \\
\hline
\end{tabular}




\begin{tabular}{|c|c|c|c|c|c|c|c|c|c|c|c|c|c|c|c|}
\hline 301 Gly & 7.74 & 108.17 & - & - & $4.21,3.55$ & - & - & - & - & 170.71 & 45.7 & - & - & - & - \\
\hline 302 Ala & 8.32 & 119.93 & - & - & 4.92 & 1.04 & - & - & - & 173.47 & 52.23 & 22.73 & - & - & - \\
\hline $303 \mathrm{Val}$ & 8.91 & 118.69 & - & - & 5.01 & 1.73 & $0.73,0.74$ & - & - & 172.92 & 59.11 & 35.98 & $22.55,20.12$ & - & - \\
\hline 304 Ser & 9.14 & 123.24 & - & - & 5.73 & $3.39,3.23$ & - & - & - & 174.25 & 56.08 & 66.61 & - & - & - \\
\hline 305 Thr & 8.52 & 112.42 & - & - & 5.41 & 4.09 & 0.99 & - & - & 174.72 & 57.85 & 70.69 & 22.88 & - & - \\
\hline 306 Phe & 8.61 & 117.36 & - & - & 5.44 & $2.72,2.45$ & - & - & - & - & 54.99 & 42.21 & - & - & - \\
\hline 307 Arg & 8.89 & - & - & 133.43 & 5.03 & $1.87,1.73$ & $1.44,1.73$ & $3.06,3.14$ & 6.76 & 174.87 & 53.53 & 33.39 & 27.02 & 43.62 & - \\
\hline 308 Asn & 8.6 & 120.59 & 111.37 & - & 4.66 & $2.79,2.61$ & - & $6.75,7.60$ & - & 174.59 & 54.72 & 38.84 & 176.69 & - & - \\
\hline 309 Lys & 8.06 & 127.38 & - & - & 3.9 & $1.41,1.62$ & $1.31,1.31$ & $1.50,1.50$ & $2.86,2.87$ & , & 58.98 & 33.81 & 25.49 & 29.76 & 42.17 \\
\hline
\end{tabular}


Table A.3: Chemical shift assignment of isolated PASc R289D. All the values are reported in ppm.

\begin{tabular}{|c|c|c|c|c|c|c|c|c|c|c|c|c|c|}
\hline \multicolumn{14}{|c|}{ PASc R289D chemical shifts [ppm] } \\
\hline & $\mathrm{H}$ & $\mathrm{N}$ & $\mathrm{HA}$ & HB & $\mathrm{HG}$ & HD & $\mathrm{HE}$ & $\mathrm{C}$ & $\mathrm{CA}$ & $\mathrm{CB}$ & $\mathrm{CG}$ & $\mathrm{CD}$ & $\mathrm{CE}$ \\
\hline 200 Pro & - & - & 4.29 & $2.27,1.86$ & $2.05,1.95$ & $3.75,3.83$ & - & 177.9 & 64.5 & 31.82 & 27.63 & 50.67 & - \\
\hline $201 \mathrm{Glu}$ & 8.46 & 119.16 & 4.21 & 2.01 & 2.24 & - & - & 177.51 & 58.32 & 29.15 & 35.47 & - & - \\
\hline 202 Glu & 8.03 & 121.13 & 4.09 & 1.98 & - & - & - & 177.67 & 57.66 & 30.15 & - & - & - \\
\hline 203 Ile & 7.99 & 120.54 & 3.83 & 1.78 & $1.11,0.76,1.33$ & 0.67 & - & 177.12 & 62.6 & 37.85 & $27.90,17.68$ & 12.66 & - \\
\hline 204 Gly & 8.24 & 109.47 & 3.84 & - & - & - & - & 175.68 & 46.61 & - & - & - & - \\
\hline 205 Leu & 7.75 & 122.01 & 4.16 & $1.70,1.53$ & 1.61 & 0.82 & - & - & 57.09 & 41.97 & 26.89 & $24.97,23.30$ & - \\
\hline 206 Leu & - & - & - & - & - & - & - & 179.32 & 57.35 & 41.77 & - & - & - \\
\hline 207 Tyr & 8.44 & 119.56 & 4.17 & $3.05,3.02$ & - & - & - & 176.96 & 61.19 & 38.27 & - & - & - \\
\hline 208 Gln & 8.04 & 118.4 & 3.85 & - & - & - & - & 178.57 & 58.64 & 28.61 & 35.48 & - & - \\
\hline 209 Glu & 8.07 & 120.84 & 3.89 & $2.09,1.99$ & $2.21,2.03$ & - & - & - & 59.58 & 29.84 & 36.63 & - & - \\
\hline 210 Lys & - & - & - & - & - & - & - & 178.27 & 59.36 & 32.09 & - & - & - \\
\hline $211 \mathrm{Gln}$ & 8.12 & 117.66 & 3.83 & 1.84 & 2.15 & - & - & 177.29 & 58.81 & 28.25 & 32.97 & - & - \\
\hline 212 Ala & 7.74 & 120.54 & 4.03 & 1.36 & - & - & - & 180.63 & 54.85 & 18.43 & - & - & - \\
\hline 213 Ile & 7.7 & 118.39 & 3.41 & 1.82 & $1.79,0.61$ & - & - & 177.11 & 65.98 & 38.13 & $29.75,17.73$ & 14.05 & - \\
\hline 214 Leu & 7.54 & 117.63 & 3.8 & - & - & - & - & 178.39 & 58.25 & 42.13 & - & - & - \\
\hline $215 \mathrm{Glu}$ & 7.86 & 114.08 & - & - & - & - & - & 177.5 & 58.23 & 30.22 & - & - & - \\
\hline 216 Ala & 7.49 & 121.61 & 4.22 & 1.35 & - & - & - & - & 52.95 & 20.42 & - & - & - \\
\hline 217 Ile & 6.97 & 115.66 & 3.63 & 1.84 & $1.80,1.04$ & 0.7 & - & 175.59 & 63.56 & 38.96 & $17.15,27.41$ & 13.71 & - \\
\hline 218 Arg & 8.48 & 124.66 & 4.09 & 1.72 & - & - & - & 178.08 & 57.85 & 30.78 & - & - & - \\
\hline 219 Glu & 8.24 & 120.91 & - & - & - & - & - & - & 58.52 & - & - & - & - \\
\hline
\end{tabular}




\begin{tabular}{|c|c|c|c|c|c|c|c|c|c|c|c|c|c|}
\hline 220 Gly & 8.64 & 106.68 & - & - & - & - & - & 172.09 & 45.22 & - & - & - & - \\
\hline $221 \mathrm{Ile}$ & 7.8 & 121.46 & 4.83 & 1.09 & $0.73,0.55$ & 0.67 & - & 174.54 & 60.3 & 42.41 & $27.08,17.45$ & 14.07 & - \\
\hline $222 \mathrm{Val}$ & 8.27 & 123.38 & - & 1.85 & 0.82 & - & - & 172.93 & 61 & 35.89 & 21.42 & - & - \\
\hline 223 Ala & 8.85 & 128.09 & 5.52 & 1.19 & - & - & - & 176.61 & 49.84 & 23.27 & - & - & - \\
\hline 224 Ile & 8.81 & 111.01 & 5.47 & 1.64 & 0.59 & 0.61 & - & 176.86 & 58.53 & 42.88 & $18.07,24.86$ & 15.49 & - \\
\hline 225 Asn & 7.87 & 119.11 & - & $3.54,2.90$ & - & - & - & 176.96 & 51.01 & 38.84 & - & - & - \\
\hline $226 \mathrm{Gln}$ & 8.02 & 115.42 & - & - & - & - & - & 176.48 & 58.9 & 28.41 & - & - & - \\
\hline 227 Glu & 7.58 & 116.8 & 4.23 & - & - & - & - & 176.99 & 56.28 & 29.73 & 36.56 & - & - \\
\hline 228 Gly & 7.94 & 106.58 & 4.25 & - & - & - & - & 172.79 & 45.35 & - & - & - & - \\
\hline $229 \mathrm{Thr}$ & 7.84 & 116.75 & 4.25 & 3.7 & 0.88 & - & - & 174.32 & 61.28 & 69.34 & 22.55 & - & - \\
\hline 230 Ile & 8.17 & 123.18 & 4.37 & 1.88 & $0.79,0.75,1.77$ & 0.69 & - & 177.53 & 63.12 & 38.37 & $29.23,18.10$ & 14.46 & - \\
\hline 231 Thr & 9.24 & 120.76 & 4.33 & 4.15 & 1.09 & - & - & 175.48 & 61.53 & 69.74 & 22.75 & - & - \\
\hline 232 Met & 7.26 & 121.82 & 4.45 & $1.63,1.99$ & $2.31,2.43$ & - & - & 173.25 & 56.64 & 35.32 & 32.64 & - & - \\
\hline $233 \mathrm{Val}$ & 8.64 & 122.48 & 5.11 & 1.98 & 0.91 & - & - & 174.02 & 60.49 & 35.26 & 21.43 & - & - \\
\hline $235 \mathrm{Gln}$ & - & - & 3.92 & $2.20,2.44$ & 2.44 & - & - & 178.77 & 59.91 & 28.13 & 34.12 & - & - \\
\hline $236 \mathrm{Thr}$ & 8.28 & 115.58 & 3.81 & 3.8 & 1.11 & - & - & 176.35 & 66.56 & 68.99 & 21.91 & - & - \\
\hline 237 Ala & 7.65 & 121.87 & 4.03 & 1.46 & - & - & - & 178.49 & 55.76 & 19.37 & - & - & - \\
\hline 238 Leu & 8.38 & 118.12 & 3.79 & $1.65,1.84$ & 1.66 & $0.62,0.59$ & - & 178.73 & 58.86 & 40.88 & 25.85 & $25.99,24.28$ & - \\
\hline 239 Lys & 7.82 & 117.48 & 4.1 & 1.91 & $1.43,1.51$ & 1.65 & 2.94 & 180.45 & 59.06 & 32.01 & 24.94 & 28.95 & 42.12 \\
\hline 240 Leu & 7.62 & 120.05 & 4.06 & $1.78,1.34$ & - & 0.73 & - & 178.33 & 57.61 & 42.24 & 27.09 & $25.14,23.59$ & - \\
\hline 241 Leu & 7.97 & 115.41 & 4.13 & $1.97,1.68$ & 1.83 & - & - & 176.94 & 55.59 & 42.99 & 26.82 & $25.19,23.08$ & - \\
\hline 242 Gly & 7.8 & 106.27 & $3.69,3.91$ & - & - & - & - & 174.21 & 45.75 & - & - & - & - \\
\hline 243 Tyr & 7.9 & 118.62 & - & $2.39,3.01$ & - & - & - & 174.89 & 57.44 & 42.63 & - & - & - \\
\hline 244 Asp & 8.47 & 117.58 & 4.57 & 2.57 & - & - & - & 176.18 & 54.27 & 41.96 & - & - & - \\
\hline 245 Asn & 7.62 & 115.61 & - & 2.72 & - & - & - & 174.99 & 52.92 & 41.33 & - & - & - \\
\hline
\end{tabular}




\begin{tabular}{|c|c|c|c|c|c|c|c|c|c|c|c|c|c|}
\hline $246 \mathrm{Glu}$ & 9.44 & 124.39 & - & - & - & - & - & 177.67 & 58.66 & 29.42 & - & - & - \\
\hline 247 Arg & 8.66 & 120.77 & 3.96 & $1.76,1.79$ & 1.58 & 3.12 & - & 176.89 & 58.69 & 29.45 & 27.17 & 43.18 & - \\
\hline 248 Asn & 7.85 & 114.39 & 4.63 & $2.60,2.91$ & - & - & - & 174.14 & 54.3 & 39.41 & - & - & - \\
\hline 250 Leu & 7.68 & 120.77 & 3.68 & $1.59,1.31$ & 1.6 & $0.77,0.64$ & - & 177.97 & 56.77 & 41.38 & 26.63 & $25.11,22.93$ & - \\
\hline 251 Gly & 8.88 & 109.61 & $3.67,3.46$ & - & - & - & - & 174.06 & 45.17 & - & - & - & - \\
\hline $252 \mathrm{Thr}$ & 7.69 & 118 & 4.56 & 4.08 & 1.19 & - & - & - & 61 & 70.19 & 21.07 & - & - \\
\hline 253 Pro & - & - & 4.5 & $1.78,2.40$ & 1.87 & $3.99,3.53$ & - & 179.51 & 63.03 & 32.63 & 28.1 & 51.81 & - \\
\hline $254 \mathrm{Ile}$ & 8.74 & 125.73 & 3.96 & 1.8 & $1.31,0.95$ & 0.84 & - & 175.86 & 63.03 & 37.61 & $18.24,29.88$ & 14.86 & - \\
\hline 255 Leu & 8.27 & 118.99 & 4.11 & 1.46 & - & $0.87,0.87$ & - & 178.33 & 56.37 & 40.47 & 26.82 & $25.33,22.74$ & - \\
\hline 256 Gln & 7.41 & 114.98 & - & - & - & - & - & 176.5 & 57.68 & 29.18 & - & - & - \\
\hline 257 Leu & 7 & 117.22 & 4.48 & $1.63,1.54$ & 1.59 & $0.81,0.84$ & - & 176.64 & 55.65 & 44.4 & 27.25 & $24.25,25.63$ & - \\
\hline 258 Ile & 8.48 & 117.05 & - & - & - & - & - & - & 58.6 & - & - & - & - \\
\hline 259 Pro & - & - & 4.51 & 1.59 & 1.88 & $3.65,3.21$ & - & 177.51 & 65.11 & 31.93 & 27.06 & 50.15 & - \\
\hline 261 Ser & 7.58 & 114 & 3.87 & $3.46,3.98$ & - & - & - & 176.56 & 59.45 & 64.33 & - & - & - \\
\hline $262 \mathrm{Arg}$ & 8.96 & 126.57 & 4.39 & 2 & 1.75 & $3.05,3.09$ & - & 177.07 & 55.26 & 29.14 & 26.83 & 42.3 & - \\
\hline 263 Leu & 8.54 & 119.87 & - & - & - & - & - & - & 59.08 & - & - & - & - \\
\hline 264 Pro & - & - & 3.97 & $2.25,1.81$ & $1.54,1.94$ & $3.27,3.47$ & - & 178.95 & 66.03 & 30.86 & 29 & 49.42 & - \\
\hline $265 \mathrm{Glu}$ & 7.29 & 118.13 & 4.21 & - & - & - & - & 178.41 & 58.54 & 29.34 & - & - & - \\
\hline $266 \mathrm{Val}$ & 7.78 & 120.08 & 3.91 & 2.04 & $0.82,0.58$ & - & - & 178.91 & 65.29 & 30.91 & $21.77,21.47$ & - & - \\
\hline 267 Ile & 7.44 & 117.73 & 3.62 & 1.9 & $0.98,0.77,1.39$ & 0.46 & - & 177.03 & 63.74 & 36.61 & $17.16,28.32$ & 12.63 & - \\
\hline 268 Arg & 7.26 & 118.55 & 3.98 & $1.90,1.92$ & 1.46 & 3.24 & - & 178.9 & 59.16 & 31.11 & 27.65 & 43.16 & - \\
\hline 269 Thr & 8.53 & 107.11 & 4.18 & 4.21 & 1.25 & - & - & 177.41 & 62.58 & 70.81 & 21.2 & - & - \\
\hline 270 Gly & 8.47 & 112.24 & $3.43,4.07$ & - & - & - & - & 172.96 & 46.39 & - & - & - & - \\
\hline 271 Gln & 8.05 & 119.72 & 4.48 & $1.78,1.93$ & 2.23 & - & - & 174.03 & 53.82 & 30.31 & 33.28 & - & - \\
\hline
\end{tabular}




\begin{tabular}{|c|c|c|c|c|c|c|c|c|c|c|c|c|c|}
\hline 272 Ala & 8.27 & 124.93 & 4.37 & 1.13 & - & - & - & - & 52.15 & 20.65 & - & - & - \\
\hline $273 \mathrm{Glu}$ & 8.63 & 120.13 & - & - & - & - & - & 174.07 & 55.31 & 32.39 & - & - & - \\
\hline 274 Tyr & 8.42 & 120.69 & 5.24 & $3.00,2.61$ & - & - & - & - & 55.82 & 40.96 & - & - & - \\
\hline 275 Asp & - & - & 4.07 & 2.69 & - & - & - & 174.81 & 55.6 & 39.3 & - & - & - \\
\hline 276 Asp & 8.9 & 119.99 & 4.66 & 2.56 & - & - & - & 175.22 & 53.16 & 40.61 & - & - & - \\
\hline $277 \mathrm{Glu}$ & 8.35 & 126.5 & 4.92 & 1.86 & 2.11 & - & - & 175.71 & 56.25 & 30.62 & 37.13 & - & - \\
\hline 278 Met & 8.91 & 123.8 & - & - & - & - & - & 173.22 & 54.49 & 36.94 & - & - & - \\
\hline $279 \mathrm{Val}$ & 8.18 & 121.42 & 4.67 & 1.71 & $0.77,0.64$ & - & - & 175.89 & 61.61 & 32.28 & $21.09,20.88$ & - & - \\
\hline 280 Leu & 8.88 & 129.78 & 4.55 & $1.14,1.23$ & 1.15 & $0.44,0.35$ & - & 176.16 & 53.78 & 45.15 & 26.88 & $24.91,25.27$ & - \\
\hline 281 Gly & 8.95 & 114.5 & $3.74,3.92$ & - & - & - & - & 175.62 & 47.33 & - & - & - & - \\
\hline 282 Gly & 8.23 & 106.48 & $3.60,4.00$ & - & - & - & - & 173.59 & 45 & - & - & - & - \\
\hline $283 \mathrm{Glu}$ & 7.83 & 121.06 & 4.62 & $1.94,1.84$ & $2.08,2.17$ & - & - & 174.89 & 54.37 & 31.85 & 35.35 & - & - \\
\hline $284 \mathrm{Thr}$ & 8.68 & 118.72 & - & 3.94 & 1.04 & - & - & 174.4 & 63.27 & 68.68 & 22.36 & - & - \\
\hline $285 \mathrm{Val}$ & 9.26 & 121.66 & 4.98 & 1.96 & $0.48,0.66$ & - & - & 173.35 & 58.49 & 35.39 & $21.01,19.07$ & - & - \\
\hline 287 Ala & 9.34 & 132.35 & 5.72 & 1.33 & - & - & - & 176.44 & 50.09 & 23.1 & - & - & - \\
\hline $288 \mathrm{Asn}$ & 8.55 & 118.02 & 5.25 & $2.35,2.96$ & - & - & - & 173.17 & 52.75 & 41.72 & - & - & - \\
\hline 289 Asp & 8.97 & 124.02 & 5.31 & $2.08,2.58$ & - & - & - & 173.6 & 53.4 & 43.18 & - & - & - \\
\hline 290 Ile & 9.28 & 123.83 & - & - & - & - & - & - & 57.55 & - & - & - & - \\
\hline 291 Pro & - & - & 4.44 & $1.62,1.89$ & $1.69,1.94$ & $3.82,3.26$ & - & 174.74 & 62.55 & 32.2 & 26.04 & 50.04 & - \\
\hline 292 Ile & 8.21 & 120.94 & 4.12 & 1.09 & $0.60,0.56,1.25$ & 0.56 & - & 175 & 60.24 & 39.37 & $26.84,18.34$ & 13.92 & - \\
\hline 293 Lys & 8.56 & 125.19 & 5.35 & 1.53 & $0.99,1.08$ & 1.53 & 2.75 & 176.76 & 54.1 & 36.62 & 26.15 & 29.57 & 42.19 \\
\hline 294 Asn & 8.38 & 118.11 & 5.05 & $3.66,2.75$ & - & - & - & - & 50.65 & 38.74 & - & - & - \\
\hline 295 Lys & 8.48 & 118.74 & 4.01 & 1.79 & 1.65 & - & 1.65 & 177.61 & 58.79 & 31.83 & 25.08 & 28.9 & 42.04 \\
\hline 296 Gln & 7.42 & 115.94 & 4.28 & 2.23 & 2.41 & - & - & 176.26 & 56.43 & 28.48 & 34.83 & - & - \\
\hline 297 Gly & 8.05 & 107.47 & 3.55 & - & - & - & - & 173.55 & 45.37 & - & - & - & - \\
\hline
\end{tabular}




\begin{tabular}{l|c|c|c|c|} 
298 Arg & 7.75 & 121.45 & 4.27 & $1.66,1.58$ \\
299 Val & 8.74 & 124.89 & 4.18 & 2.1 \\
300 Ile & 8.65 & 120.06 & 4.56 & 2.16 \\
301 Gly & 7.71 & 108.34 & - & - \\
302 Ala & 8.33 & 120.58 & 4.95 & 1.07 \\
303 Val & 9.33 & 119.09 & 5.15 & 1.76 \\
304 Ser & 9.25 & 124.38 & 5.93 & 3.38 \\
305 Thr & 8.48 & 113.28 & 5.58 & 4.02 \\
306 Phe & 8.64 & 117.58 & 5.56 & 2.48 \\
307 Arg & - & - & 4.97 & $1.78,1.82$ \\
308 Asn & 8.58 & 120.45 & 4.65 & $2.59,2.80$ \\
309 Lys & 8.1 & 127.97 & 3.88 & $1.36,1.60$
\end{tabular}

\begin{tabular}{c|c|c|}
$1.58,1.43$ & $3.07,3.13$ & - \\
$1.12,1.00$ & - & - \\
$0.70,0.76,0.89$ & 0.68 & - \\
- & - & - \\
- & - & - \\
$0.78,0.77$ & - & - \\
- & - & - \\
1.08 & - & - \\
- & - & - \\
$1.72,1.45$ & $3.08,3.13$ & - \\
- & - & - \\
1.31 & 1.5 & 2.87
\end{tabular}

\begin{tabular}{c|c|c|c|c|c|}
175.96 & 54.97 & 30.85 & 27.24 & 43.22 & - \\
176.7 & 63.8 & 31.29 & $22.80,23.15$ & - & - \\
175.24 & 60.7 & 39.03 & $18.16,26.38$ & 13.75 & - \\
170.59 & 45.55 & - & - & - & - \\
173.2 & 52.09 & 23.26 & - & - & - \\
172.4 & 58.85 & 36.06 & $19.57,22.93$ & - & - \\
174.59 & 57.1 & 66.54 & - & - & - \\
- & 58.08 & 70.31 & 22.4 & - & - \\
- & 55.23 & 42.16 & - & - & - \\
174.83 & 53.63 & 33.49 & 26.82 & 43.49 & - \\
174.6 & 54.84 & 38.72 & - & - & - \\
- & 59.08 & 33.86 & 25.57 & 29.86 & 42.15
\end{tabular}


Table A.4: Chemical shift assignment of isolated PASc N288D. All the values are reported in ppm.

\begin{tabular}{|c|c|c|c|c|c|c|c|c|c|c|c|c|c|}
\hline \multicolumn{14}{|c|}{ PASc N288D chemical shifts [ppm] } \\
\hline & $\mathrm{H}$ & $\mathrm{N}$ & HA & $\mathrm{HB}$ & $\mathrm{HG}$ & $\mathrm{HD}$ & $\mathrm{HE}$ & $\mathrm{C}$ & $\mathrm{CA}$ & $\mathrm{CB}$ & $\mathrm{CG}$ & $\mathrm{CD}$ & $\mathrm{CE}$ \\
\hline 200 Pro & - & - & 4.34 & $1.89,2.31$ & $1.99,2.08$ & $3.78,3.86$ & - & 177.86 & 64.59 & 31.96 & 27.67 & 50.81 & - \\
\hline $201 \mathrm{Glu}$ & 8.47 & 119.17 & 4.15 & 2.01 & 2.25 & - & - & 177.46 & 57.94 & 29.68 & 36.46 & - & - \\
\hline 202 Glu & 8.04 & 121.08 & - & 2.04 & - & - & - & 177.59 & 57.66 & 29.92 & 36.72 & - & - \\
\hline 203 Ile & 7.97 & 120.42 & 3.9 & 1.83 & $0.80,1.14,1.38$ & 0.71 & - & 177.1 & 62.68 & 38.15 & $17.66,27.98$ & 12.72 & - \\
\hline 204 Gly & 8.23 & 109.57 & - & - & - & - & - & 175.56 & 46.55 & - & - & - & - \\
\hline 205 Leu & 7.79 & 121.99 & 4.2 & - & 1.64 & - & - & - & 56.95 & 41.94 & 27.05 & $25.01,23.56$ & - \\
\hline 206 Leu & - & - & - & - & - & - & - & 179.1 & - & - & - & - & - \\
\hline $207 \mathrm{Tyr}$ & 8.36 & 119.44 & 4.23 & 3.06 & - & - & - & 177.06 & 61.15 & 38.37 & - & - & - \\
\hline 208 Gln & 8.08 & 118.74 & - & - & - & - & - & 178.47 & 58.58 & 28.66 & - & - & - \\
\hline 209 Glu & 8.26 & 120.98 & - & - & - & - & - & 177.8 & 59.61 & 29.69 & - & - & - \\
\hline 210 Lys & 7.88 & 117.28 & 4.12 & $1.96,1.83$ & $1.53,1.46$ & 1.68 & 2.97 & 178.18 & 59.48 & 32.06 & 25.07 & 29.07 & 42.27 \\
\hline $211 \mathrm{Gln}$ & 8.07 & 117.58 & - & - & - & - & - & 177.29 & 58.83 & 28.26 & - & - & - \\
\hline 212 Ala & 7.78 & 120.31 & 4.07 & 1.42 & - & - & - & 180.72 & 54.94 & 18.53 & - & - & - \\
\hline 213 Ile & 7.77 & 118.4 & 3.46 & 1.85 & $1.81,0.75,0.71$ & 0.64 & - & 177.11 & 65.89 & 38.08 & $17.94,29.46$ & 14.14 & - \\
\hline 214 Leu & 7.5 & 117.71 & - & - & - & - & - & 177.97 & 58.14 & 42.15 & - & - & - \\
\hline $215 \mathrm{Glu}$ & 7.71 & 113.57 & - & - & - & - & - & 177.62 & 57.51 & 30.03 & - & - & - \\
\hline 216 Ala & 7.58 & 122.04 & 4.28 & 1.38 & - & - & - & 177.55 & 53.11 & 20.11 & - & - & - \\
\hline 217 Ile & 7.01 & 113.97 & 3.82 & - & 0.96 & 0.72 & - & 175.51 & 62.98 & 38.67 & $17.39,26.31$ & 13.85 & - \\
\hline 218 Arg & 8.51 & 123.64 & 4.15 & - & - & 2.9 & - & 177.77 & 57.98 & 30.68 & 27.3 & 43.92 & - \\
\hline $219 \mathrm{Glu}$ & 7.82 & 118.8 & - & - & - & - & - & 175.93 & 56.59 & 29.65 & - & - & - \\
\hline
\end{tabular}




\begin{tabular}{|c|c|c|c|c|c|c|c|c|c|c|c|c|c|}
\hline 220 Gly & 9.03 & 107.74 & - & - & - & - & - & 172.1 & 45.57 & - & - & - & - \\
\hline $221 \mathrm{Ile}$ & 8.01 & 122.47 & 4.88 & 1.23 & 0.59 & 0.69 & - & 174.11 & 60.19 & 42.24 & $27.38,17.73$ & 14 & - \\
\hline $222 \mathrm{Val}$ & 8.28 & 123.31 & - & 1.88 & - & - & - & 172.79 & 61.07 & 35.79 & - & - & - \\
\hline 223 Ala & 8.84 & 127.92 & 5.57 & 1.22 & - & - & - & 176.58 & 49.88 & 23.3 & - & - & - \\
\hline 224 Ile & 8.76 & 110.91 & 5.54 & 1.71 & - & 0.64 & - & 176.87 & 58.48 & 42.84 & $24.79,17.84$ & 15.09 & - \\
\hline 225 Asn & 7.92 & 119.18 & 5.1 & $2.94,3.62$ & - & - & - & 176.98 & 51.01 & 38.92 & - & - & - \\
\hline 226 Gln & 8.01 & 115.39 & - & - & - & - & - & 176.55 & 58.93 & 28.3 & - & - & - \\
\hline $227 \mathrm{Glu}$ & 7.58 & 116.75 & 4.29 & 2.02 & 2.21 & - & - & 176.99 & 56.55 & 29.4 & 36.65 & - & - \\
\hline 228 Gly & 8 & 106.77 & - & - & - & - & - & 172.76 & 45.38 & - & - & - & - \\
\hline $229 \mathrm{Thr}$ & 7.87 & 117.69 & 4.22 & 3.76 & 0.92 & - & - & 174.17 & 61.6 & 69.28 & 22.9 & - & - \\
\hline 230 Ile & 8.25 & 123.79 & 4.43 & 1.96 & - & - & - & 177.41 & 63.14 & 38.23 & $29.28,17.92$ & 14.67 & - \\
\hline $231 \mathrm{Thr}$ & 9.2 & 120.97 & 4.35 & 4.19 & 1.12 & - & - & 175.45 & 61.66 & 69.62 & 23.07 & - & - \\
\hline 232 Met & 7.28 & 121.47 & 4.5 & $2.00,1.68$ & 2.4 & - & - & 173.16 & 56.61 & 35.69 & 32.64 & - & - \\
\hline $233 \mathrm{Val}$ & 8.55 & 121.87 & 5.17 & 2.07 & $0.98,0.92$ & - & - & 173.81 & 60.43 & 35.31 & $21.26,21.45$ & - & - \\
\hline 234 Asn & 7.88 & 121.74 & 5.22 & $3.68,2.51$ & - & - & - & 175.61 & 50.56 & 40.47 & - & - & - \\
\hline $235 \mathrm{Gln}$ & 9.19 & 119.61 & - & - & - & - & - & 178.84 & 59.86 & 28.14 & - & - & - \\
\hline $236 \mathrm{Thr}$ & 8.21 & 115.68 & 3.86 & 3.84 & 1.1 & - & - & 176.26 & 66.93 & 68.57 & 22.33 & - & - \\
\hline 237 Ala & 7.71 & 121.96 & 4.03 & 1.51 & - & - & - & 178.51 & 55.79 & 19.06 & - & - & - \\
\hline 238 Leu & 8.27 & 117.65 & 3.84 & 1.92 & - & 0.64 & - & 178.73 & 58.99 & 40.77 & 25.94 & $24.35,24.14$ & - \\
\hline 239 Lys & 7.85 & 117.98 & - & - & - & - & - & 180.5 & 59.31 & 32.02 & - & - & - \\
\hline 240 Leu & 7.71 & 120.27 & - & - & - & - & - & 178.28 & 57.62 & 42.33 & - & - & - \\
\hline 241 Leu & 7.91 & 115.23 & - & - & - & - & - & 176.91 & 55.51 & 43.1 & - & - & - \\
\hline 242 Gly & 7.81 & 106.57 & - & - & - & - & - & 174.24 & 45.8 & - & - & - & - \\
\hline $243 \mathrm{Tyr}$ & 8 & 119.03 & - & $3.06,2.45$ & - & - & - & 174.9 & 57.51 & 42.52 & - & - & - \\
\hline 244 Asp & 8.44 & 117.82 & 4.59 & 2.6 & - & - & - & 176.12 & 54.33 & 42.02 & - & - & - \\
\hline 245 Asn & 7.67 & 115.56 & 4.75 & $2.75,2.80$ & - & - & - & 174.94 & 53.06 & 41.09 & - & - & - \\
\hline $246 \mathrm{Glu}$ & 9.38 & 123.51 & - & - & - & - & - & 177.53 & 58.41 & 29.19 & - & - & - \\
\hline
\end{tabular}




\begin{tabular}{|c|c|c|c|c|}
\hline 247 Arg & 8.57 & 121.14 & - & - \\
\hline 248 Asn & 7.99 & 115.14 & - & $2.94,2.65$ \\
\hline 249 Val & 7.53 & 115.07 & 3.91 & 1.68 \\
\hline 250 Leu & 7.76 & 120.66 & 3.72 & - \\
\hline 251 Gly & 8.88 & 109.85 & - & - \\
\hline 252 Thr & 7.71 & 117.78 & 4.62 & 4.14 \\
\hline 253 Pro & - & - & 4.58 & $1.81,2.49$ \\
\hline 254 Ile & 8.8 & 126.27 & 4.02 & - \\
\hline 255 Leu & 8.23 & 119.09 & - & - \\
\hline $256 \mathrm{Gln}$ & 7.43 & 114.93 & - & - \\
\hline 257 Leu & 7.04 & 117.85 & 4.47 & $1.69,1.59$ \\
\hline 258 Ile & 8.52 & 117.29 & 4.35 & 1.85 \\
\hline 259 Pro & - & - & 4.6 & 1.76 \\
\hline 260 His & 8.62 & 112.61 & - & $3.21,3.09$ \\
\hline $261 \mathrm{Ser}$ & 7.69 & 114.83 & 4.17 & $4.08,3.57$ \\
\hline 264 Pro & - & - & 4 & 1.81 \\
\hline $265 \mathrm{Glu}$ & 7.26 & 117.66 & - & - \\
\hline $266 \mathrm{Val}$ & 7.5 & 119.24 & - & - \\
\hline 267 Ile & 7.14 & 118 & 3.62 & 1.99 \\
\hline $268 \mathrm{Arg}$ & 7.16 & 117.82 & - & - \\
\hline $269 \mathrm{Thr}$ & 8.5 & 107.15 & 4.21 & 4.24 \\
\hline 270 Gly & 8.39 & 111.85 & - & - \\
\hline 271 Gln & 7.98 & 119.45 & - & - \\
\hline 272 Ala & 8.3 & 125.54 & 4.41 & 1.2 \\
\hline $273 \mathrm{Glu}$ & 8.65 & 119.46 & - & - \\
\hline 274 Tyr & 8.48 & 120.34 & 5.36 & $3.01,2.56$ \\
\hline 275 Asp & 9.05 & 117.87 & 4.03 & 2.78 \\
\hline
\end{tabular}

\begin{tabular}{|c|c|c|c|c|c|c|c|c|}
\hline - & - & - & 176.85 & 58.56 & 29.6 & - & - & - \\
\hline - & - & - & 174.21 & 54.1 & 39.27 & - & - & - \\
\hline $0.51,0.39$ & - & - & 176.1 & 62.77 & 33.98 & $21.96,21.19$ & - & - \\
\hline - & - & - & 178.05 & 56.81 & 41.34 & 26.75 & $23.06,25.16$ & - \\
\hline - & - & - & 173.99 & 45.27 & - & - & - & - \\
\hline 1.21 & - & - & - & 60.95 & 70.38 & 21.32 & - & - \\
\hline $2.10,1.92$ & $3.57,4.01$ & - & 179.52 & 63.08 & 32.64 & 28.12 & 51.88 & - \\
\hline 1.02 & 0.9 & - & 175.86 & 63.29 & 37.72 & $17.96,30.07$ & 14.82 & - \\
\hline - & - & - & 178.34 & 56.44 & - & - & - & - \\
\hline - & - & - & 176.44 & 57.55 & 29.07 & - & - & - \\
\hline- & 0.81 & - & 176.89 & 56.02 & 44.12 & 27.31 & $24.46,25.37$ & - \\
\hline 0.52 & 0.85 & - & - & 58.68 & 38.53 & $26.04,18.02$ & 13.56 & - \\
\hline 1.98 & $3.34,3.74$ & - & 177.29 & 65.36 & 31.89 & 27.12 & 50.19 & - \\
\hline - & - & - & 175.18 & 54.61 & 28.8 & - & - & - \\
\hline - & - & - & - & 59.41 & 64.64 & - & - & - \\
\hline 1.54 & 3.49 & - & 178.85 & 65.86 & 30.9 & 29.06 & 49.8 & - \\
\hline - & - & - & 178.36 & 58.57 & 29.1 & - & - & - \\
\hline - & - & - & 178.52 & 65.12 & 31.07 & - & - & - \\
\hline - & 0.49 & - & 176.9 & 63.54 & 36.55 & $28.38,17.06$ & 12.68 & - \\
\hline - & - & - & 178.84 & 59.25 & 31.04 & - & - & - \\
\hline 1.28 & - & - & 177.36 & 62.7 & 70.64 & 21.35 & - & - \\
\hline - & - & - & 173.06 & 46.44 & - & - & - & - \\
\hline - & - & - & 174.11 & 54.03 & 30.18 & - & - & - \\
\hline - & - & - & 176.63 & 52.04 & 20.73 & - & - & - \\
\hline - & - & - & 174.07 & 54.94 & 32.62 & - & - & - \\
\hline- & - & - & 176.21 & 55.89 & 41.91 & - & - & - \\
\hline- & - & - & 174.97 & 55.77 & 39.24 & - & - & - \\
\hline
\end{tabular}




\begin{tabular}{|c|c|c|c|c|c|c|c|c|c|c|c|c|c|}
\hline 276 Asp & 9.06 & 120.72 & 4.88 & $2.46,2.74$ & - & - & - & 175.75 & 53.68 & 42.46 & - & - & - \\
\hline $277 \mathrm{Glu}$ & 8.32 & 126.73 & - & - & - & - & - & 175.7 & 56.77 & 30.73 & - & - & - \\
\hline 278 Met & 9.16 & 124.66 & - & - & - & - & - & 172.94 & 54.57 & 36.92 & - & - & - \\
\hline 279 Val & 8.26 & 121.3 & 4.76 & 1.76 & $0.67,0.81$ & - & - & 175.86 & 61.62 & 32.48 & $20.99,21.14$ & - & - \\
\hline 280 Leu & 8.89 & 129.6 & 4.59 & $1.29,1.12$ & 1.13 & 0.35 & - & 176.19 & 53.89 & 45.32 & 26.8 & 24.93 & - \\
\hline 281 Gly & 8.98 & 114.82 & - & - & - & - & - & 175.62 & 47.37 & - & - & - & - \\
\hline 282 Gly & 8.22 & 106.83 & - & - & - & - & - & 173.55 & 45.03 & - & - & - & - \\
\hline $283 \mathrm{Glu}$ & 7.83 & 121.05 & - & - & - & - & - & 174.85 & 54.42 & 31.79 & - & - & - \\
\hline $284 \mathrm{Thr}$ & 8.66 & 118.93 & - & 3.98 & 1.09 & - & - & 174.28 & 63.45 & 68.9 & 22.37 & - & - \\
\hline $285 \mathrm{Val}$ & 9.23 & 121.49 & 4.97 & 2.07 & $0.73,0.64$ & - & - & 173.34 & 58.55 & 35.67 & $19.29,21.50$ & - & - \\
\hline 286 Ile & 9.02 & 119.87 & 4.6 & 1.67 & $1.39,1.11,0.81$ & 0.75 & - & 175.69 & 59.9 & 38.49 & $27.19,18.12$ & 12.09 & - \\
\hline 287 Ala & 9.41 & 132.26 & 5.82 & 1.32 & - & - & - & 176.06 & 50.45 & 23.05 & - & - & - \\
\hline 288 Asp & 8.13 & 119.44 & 5.39 & $2.91,2.48$ & - & - & - & 175.54 & 52.8 & 43.91 & - & - & - \\
\hline $289 \mathrm{Arg}$ & 9.15 & 119.46 & 5.26 & 1.34 & - & - & - & 174.67 & 54.79 & 34.22 & 27.52 & 43.4 & - \\
\hline 290 Ile & 9.12 & 122.67 & 4.61 & 1.72 & 1.05 & 0.91 & - & - & 57.98 & 42.88 & $27.73,17.62$ & 14.52 & - \\
\hline 291 Pro & - & - & 4.5 & $1.69,1.96$ & $1.70,1.97$ & $3.84,3.26$ & - & 174.73 & 62.49 & 32.42 & 26 & 50.42 & - \\
\hline 292 Ile & 8.23 & 121.32 & 4.17 & 1.17 & $0.65,1.27$ & - & - & 174.93 & 60.2 & 39.38 & $26.76,18.08$ & 13.29 & - \\
\hline 293 Lys & 8.55 & 125.22 & 5.4 & 1.58 & $1.12,1.05$ & 1.58 & 2.8 & 176.76 & 54.3 & 36.54 & 26.31 & 29.65 & 42.33 \\
\hline 294 Asn & 8.37 & 118.01 & 4.77 & $3.69,2.79$ & - & - & - & 177.85 & 50.58 & 38.82 & - & - & - \\
\hline 295 Lys & 8.49 & 118.7 & - & - & - & - & - & 177.57 & 58.88 & 31.81 & - & - & - \\
\hline $296 \mathrm{Gln}$ & 7.43 & 115.89 & - & - & - & - & - & 176.25 & 56.5 & 28.55 & - & - & - \\
\hline 297 Gly & 8.06 & 107.45 & - & - & - & - & - & 173.53 & 45.42 & - & - & - & - \\
\hline $298 \mathrm{Arg}$ & 7.77 & 121.45 & 4.32 & $1.69,1.62$ & $1.61,1.47$ & 3.15 & - & 175.93 & 55.09 & 30.97 & 27.34 & 43.33 & - \\
\hline $299 \mathrm{Val}$ & 8.75 & 124.87 & 4.19 & 2.15 & $1.02,1.16$ & - & - & 176.65 & 63.97 & 31.34 & $23.53,22.74$ & - & - \\
\hline 300 Ile & 8.67 & 120.05 & 4.6 & 2.2 & 0.83 & 0.71 & - & 175.29 & 60.54 & 39.11 & $18.28,26.46$ & 13.89 & - \\
\hline 301 Gly & 7.73 & 108.27 & - & - & - & - & - & 170.74 & 45.69 & - & - & - & - \\
\hline 302 Ala & 8.33 & 120.09 & 4.97 & 1.05 & - & - & - & 173.37 & 52.13 & 22.7 & - & - & - \\
\hline
\end{tabular}




\begin{tabular}{l|c|c|c|c|}
303 Val & 9.04 & 118.76 & 5.17 & 1.76 \\
304 Ser & 9.21 & 124.4 & 5.74 & $3.44,3.25$ \\
305 Thr & 8.53 & 113.47 & 5.33 & 4.07 \\
306 Phe & 8.78 & 118.33 & 5.48 & 2.5 \\
307 Arg & 9 & 122.53 & 4.98 & - \\
308 Asn & 8.58 & 120.24 & - & 2.65 \\
309 Lys & 8.04 & 127.18 & - & -
\end{tabular}

\begin{tabular}{c|c|c|}
$0.80,0.82$ & - & - \\
- & - & - \\
1.03 & - & - \\
- & - & - \\
$1.53,1.75$ & 3.14 & - \\
- & - & - \\
- & - & -
\end{tabular}

\begin{tabular}{c|c|c|c|c|c|}
172.87 & 59.05 & 36.15 & $20.08,22.86$ & - & - \\
173.83 & 56.51 & 66.67 & - & - & - \\
174.17 & 57.88 & 70.44 & 21.97 & - & - \\
174.9 & 55.03 & 42.27 & - & - & - \\
175.08 & 54.12 & 33.18 & 27.3 & 43.44 & - \\
174.55 & 54.55 & 39.15 & - & - & - \\
- & 58.76 & - & - & - & -
\end{tabular}


Table A.5: Backbone chemical shift assignments of citrate-bound CitApc C12AR93A. All the values are reported in $\mathrm{ppm}$.

\begin{tabular}{|c|c|c|c|c|c|}
\hline \multicolumn{6}{|c|}{ Citrate-bound CitApc C12AR93A chemical shifts [ppm] } \\
\hline & $\mathrm{H}[]$ & $\mathrm{N}$ & $\mathrm{C}$ & $\mathrm{CA}$ & $\mathrm{CB}$ \\
\hline 29 Met & - & - & - & 58.43 & 31.75 \\
\hline 30 Phe & 8.30 & 116.33 & 175.94 & 59.79 & 38.7 \\
\hline 31 Ala & 8.77 & 121.73 & 177.78 & 55.4 & 17.42 \\
\hline 32 Ala & 8.09 & 120.71 & - & 54.84 & 17.59 \\
\hline 38 Ile & 7.24 & 121.93 & 177.41 & 63.71 & 36.19 \\
\hline 39 Gly & 9.58 & 115.27 & 173.65 & 44.66 & - \\
\hline 40 Met & 7.8 & 118.51 & - & 58.92 & - \\
\hline $41 \mathrm{Arg}$ & - & - & - & 58.86 & - \\
\hline 42 Ala & 8.62 & 121.27 & 179.71 & 55.24 & 17.32 \\
\hline 43 Leu & 8.73 & 117.49 & 178.21 & 57.36 & 40.07 \\
\hline 44 Asn & 8.88 & 116.89 & 173.55 & 56.09 & 38.26 \\
\hline $45 \mathrm{Val}$ & 8.12 & 120.93 & 177.28 & 67.44 & 30.71 \\
\hline 46 Ala & 8.81 & 123.92 & 179.82 & 55.69 & 17.31 \\
\hline $47 \mathrm{Glu}$ & 9.13 & 114.29 & 179.93 & 58.22 & 29.5 \\
\hline $48 \mathrm{Thr}$ & 8.08 & 117.83 & 177.61 & 66.43 & 67.99 \\
\hline $49 \mathrm{Val}$ & - & - & 178.72 & 67.22 & 31.05 \\
\hline 50 Ala & 8.78 & 118.63 & 178.22 & 54.57 & 17.97 \\
\hline 51 Ser & 7.4 & 111.62 & 174.31 & 59.22 & 64.21 \\
\hline $52 \mathrm{Thr}$ & 7.84 & 115.03 & - & 62.82 & 70.99 \\
\hline 54 Leu & - & - & 177.47 & 57.97 & 41.3 \\
\hline $55 \mathrm{Val}$ & 6.91 & 114.58 & 175.83 & 65 & 30.21 \\
\hline $56 \operatorname{Arg}$ & 6.8 & 115.05 & 180.17 & 59.15 & 29.12 \\
\hline 57 Glu & 8.56 & 118.06 & 179.76 & 58.7 & 28.56 \\
\hline 58 Ala & 8.41 & 121.39 & 179.35 & 53.84 & 17.21 \\
\hline 59 Phe & 7.5 & 114.32 & 177.05 & 61.2 & 38.29 \\
\hline 60 Arg & 7.8 & 116.81 & 176.78 & 55.94 & 29.46 \\
\hline 61 Asp & 7.43 & 120.93 & 176.1 & 53.73 & 40.46 \\
\hline 62 Ser & 8.69 & 116.06 & 175.52 & 61.03 & 61.94 \\
\hline 63 Asn & 8.11 & 114.08 & 174.16 & 49.58 & 38 \\
\hline
\end{tabular}




\begin{tabular}{|c|c|c|c|c|c|}
\hline 64 Pro & - & - & 176.1 & 64.43 & 32.71 \\
\hline 65 Ser & 7.56 & 107.3 & 176 & 61.91 & 63.62 \\
\hline $66 \mathrm{Val}$ & 7.25 & 118.94 & 178.58 & 66.31 & 29.87 \\
\hline 67 Arg & 8.3 & 113.97 & 179.27 & 57.61 & 31.52 \\
\hline 68 Leu & 7.97 & 116.48 & 177.44 & 57.5 & 42.29 \\
\hline $69 \mathrm{Gln}$ & 8.72 & 119.2 & 174.92 & 60.11 & 23.94 \\
\hline 70 Pro & - & - & 180.11 & 65.27 & 29.72 \\
\hline 71 Phe & 6.65 & 116.89 & 176.14 & 59.9 & 39.02 \\
\hline 72 Ala & 8.88 & 121.98 & 179.62 & 55.24 & 17.38 \\
\hline $73 \mathrm{Glu}$ & 8.16 & 115.61 & 178.77 & 57.39 & 29.5 \\
\hline 74 Arg & 7.62 & 121.05 & 178.48 & 58.61 & 28.62 \\
\hline 75 Ile & 7.83 & 117.2 & 179.69 & 61.74 & 33.8 \\
\hline 76 Arg & 8.98 & 125.03 & 177.99 & 60.52 & 28.35 \\
\hline $79 \mathrm{Thr}$ & - & - & - & 62.77 & 71.13 \\
\hline 80 Gly & 7.79 & 108.79 & 174.04 & 45.13 & - \\
\hline 81 Ala & 7.1 & 121.54 & 175.67 & 51.13 & 17.33 \\
\hline $82 \mathrm{Glu}$ & 8.04 & 120.88 & 176.59 & 57.33 & 28.14 \\
\hline 83 Tyr & 6.4 & 104.25 & 172.76 & 54.42 & 38.38 \\
\hline $84 \mathrm{Val}$ & 7.86 & 120.81 & 174.22 & 62.14 & 31.09 \\
\hline $85 \mathrm{Val}$ & 9.78 & 126.77 & 175.95 & 60.56 & 33.4 \\
\hline 86 Ile & 7.48 & 124.27 & 175.42 & 59.41 & 39.37 \\
\hline 87 Gly & 9.78 & 115.67 & 172.88 & 43.81 & - \\
\hline 88 Asn & 7.98 & 116.42 & 176.54 & 50.65 & 38.27 \\
\hline 89 Arg & 7.42 & 115.45 & 176.21 & 58.18 & 28.99 \\
\hline 90 Gln & 7.33 & 115.41 & 176.1 & 55.47 & 27.83 \\
\hline 91 Gly & 8.2 & 107.76 & 172.71 & 45.18 & - \\
\hline $92 \mathrm{Ile}$ & 7.49 & 119.85 & 175.79 & 57.92 & 35.23 \\
\hline 93 Ala & 7.77 & 125.41 & 176.96 & 52.14 & 18.09 \\
\hline 94 Tyr & 9.79 & 122.37 & 174.75 & 55.44 & 39.73 \\
\hline 95 Ala & 7.85 & 119.95 & 175.32 & 51.14 & 21.47 \\
\hline $96 \mathrm{His}$ & 9.05 & 123.62 & 172.59 & 55.71 & 33.66 \\
\hline 97 Pro & - & - & 178.07 & 64.28 & 30.82 \\
\hline 98 Leu & 10.96 & 123.09 & 177.34 & 52.93 & 39.94 \\
\hline
\end{tabular}




\begin{tabular}{|c|c|c|c|c|c|}
\hline 99 Thr & 8.48 & 120.39 & 177.84 & 65.72 & 67.76 \\
\hline $100 \mathrm{Glu}$ & 9.33 & 119.61 & 176.58 & 57.83 & 27.91 \\
\hline $101 \mathrm{Arg}$ & 7.96 & 119.86 & 178.57 & 52.58 & 28.28 \\
\hline 102 Ile & 7.26 & 121.92 & 177.43 & 63.76 & 36.28 \\
\hline 103 Gly & 9.59 & 115.26 & 173.66 & 44.67 & - \\
\hline 104 Lys & 7.78 & 118.42 & 176.7 & 53.48 & 34.06 \\
\hline 105 Ser & 8.32 & 116.9 & 173.62 & 59.73 & 63.43 \\
\hline 106 Met & 8.75 & 122.35 & 176.51 & 56.31 & 34.9 \\
\hline 107 Ile & 10.22 & 129.6 & 175.33 & 59.46 & 39.08 \\
\hline 108 Gly & 8.25 & 112.19 & 176.16 & 44.73 & - \\
\hline 109 Gly & 8.94 & 108.52 & 174.97 & 46.28 & - \\
\hline 110 Asp & 8.12 & 116.43 & 176.61 & 51.97 & 37.88 \\
\hline 111 Asn & 8.39 & 115.08 & 177.75 & 53.96 & 38.45 \\
\hline 112 Lys & 7.91 & 120.04 & 179 & 60.81 & 31.63 \\
\hline 113 Glu & 9 & 115.41 & 178.98 & 58.28 & 28.44 \\
\hline 114 Val & 6.99 & 119.79 & 180.54 & 63.06 & 30.22 \\
\hline 115 Leu & 7.59 & 118.14 & 178.07 & 56.5 & 40.19 \\
\hline 116 Lys & 7.03 & 115.95 & 176.91 & 56.22 & 31.75 \\
\hline 117 Gly & 8.39 & 107.28 & 173.7 & 45.03 & - \\
\hline 118 Lys & 7.81 & 122.46 & 174.12 & 54.85 & 32.85 \\
\hline 119 Ser & 8.08 & 116.31 & 175.18 & 57.24 & 63.62 \\
\hline 120 Ile & 8.94 & 116.91 & 178.72 & 59.11 & 42.19 \\
\hline 121 Ile & 8.12 & 120.99 & 176.2 & 60.71 & 38.54 \\
\hline 122 Ser & 9.45 & 122.12 & 173.7 & 55.19 & 64.85 \\
\hline $123 \mathrm{Glu}$ & 8.48 & 128.29 & 175.28 & 54.68 & 30.2 \\
\hline 124 Ala & 8.73 & 126.32 & 174.8 & 50.6 & 21.49 \\
\hline $125 \mathrm{Val}$ & 8.58 & 119.79 & 175.5 & 61.98 & 29.94 \\
\hline 126 Gly & 6.75 & 112.12 & 176.91 & 44.32 & - \\
\hline 127 Ser & 7.25 & 120.77 & 176.75 & 62.69 & 61.92 \\
\hline 128 Leu & 11.07 & 123.21 & 176.76 & 54.8 & 40.71 \\
\hline 129 Gly & 7.21 & 104.76 & 172.25 & 43.63 & - \\
\hline 130 Pro & - & - & 177.29 & 63.35 & 31.31 \\
\hline $131 \mathrm{Ala}$ & 9.5 & 129.49 & 175.13 & 52.08 & 24.0 \\
\hline
\end{tabular}




\begin{tabular}{|c|c|c|c|c|c|}
\hline 132 Ile & 8.53 & 117.72 & 174.06 & 59.35 & 39.96 \\
\hline 133 Arg & 9.41 & 122.88 & 175.27 & 54.03 & 33.04 \\
\hline 34 Gly & 9.16 & 110.56 & 171.93 & 43.8 & - \\
\hline 35 Lys & 9.16 & 122.84 & 173.62 & 54.08 & 33.24 \\
\hline 136 Ala & 8.91 & 122.79 & 173.92 & 47.46 & 21.16 \\
\hline 37 Pro & - & - & 174.69 & 60.62 & 30.04 \\
\hline 38 Ile & 7.51 & 118.09 & 175.24 & 59.62 & 38.78 \\
\hline 39 Phe & 8.72 & 125.93 & 177.12 & 56.47 & 41.51 \\
\hline 10 Asp & 8.93 & 119.02 & 178.26 & 51.68 & 40.55 \\
\hline $11 \mathrm{Glu}$ & 9.59 & 117.95 & 176.76 & 58.58 & 27.93 \\
\hline Asn & 8.2 & 116.07 & 175.84 & 52.47 & 39.01 \\
\hline 3 Gly & 8.03 & 107.75 & 174.35 & 45.36 & - \\
\hline Ser & 8.62 & 118.33 & 174.05 & 57.62 & 62.99 \\
\hline $45 \mathrm{Val}$ & 8.85 & 124.55 & 177.38 & 63.17 & 30.57 \\
\hline 16 Ile & 8.89 & 120.69 & 175.24 & 60.51 & 38.92 \\
\hline 17 Gly & 7.25 & 106.58 & 170.12 & 45.68 & - \\
\hline Ile & 8.64 & 118.93 & 174.19 & 60.14 & 44.55 \\
\hline Val & 9.15 & 124.76 & 174.26 & 60.74 & 32.75 \\
\hline 50 Ser & 9.54 & 122.26 & 173.86 & 54.97 & 65.04 \\
\hline $51 \mathrm{Val}$ & 9.08 & 128.75 & 174.92 & 60.06 & 31.88 \\
\hline 2 Gly & 7.04 & 112.1 & 171.14 & 43.33 & - \\
\hline 153 Phe & 8.5 & 116.63 & 175.19 & 56.76 & 42.03 \\
\hline 54 Leu & 9.58 & 120.44 & 179.37 & 54.98 & 39.41 \\
\hline 155 Leu & 8.84 & 123.74 & 177.88 & 57.44 & 39.79 \\
\hline 56 Glu & 7.93 & 120.04 & 177.88 & 60.72 & 28.29 \\
\hline 157 Asp & 8.04 & 116.53 & 179.06 & 58.44 & 38.2 \\
\hline 160 Arg & - & - & 178.41 & 60.37 & 29.26 \\
\hline 161 Thr & 8.44 & 120.12 & 177.74 & 65.6 & 67.82 \\
\hline 00 Pro & - & - & 178.35 & 62.55 & 29.02 \\
\hline 201 Glu & 8.4 & 117.59 & 177.87 & 57.72 & 28.47 \\
\hline 202 Glu & 8.04 & 121.18 & - & - & - \\
\hline 3 Ile & - & - & 178 & 64.88 & - \\
\hline 4 Gly & 8.41 & 107.5 & 174.83 & 47.59 & - \\
\hline
\end{tabular}




\begin{tabular}{l|c|c|c|c|c}
205 Leu & 8.32 & 119.3 & 177.75 & 57.75 & 39.98 \\
213 Ile & - & - & 176.98 & 60.75 & - \\
213 Ile & - & - & - & - & 41.43 \\
214 Leu & - & - & - & 55.37 & - \\
214 Leu & 9.81 & 122.17 & 175.36 & - & 39.83 \\
215 Glu & 9 & 117.64 & 179.81 & - & 28.63 \\
215 Glu & - & - & - & 58.86 & - \\
216 Ala & 8.4 & 121.4 & 179.35 & 53.85 & - \\
216 Ala & - & - & - & - & 17.24 \\
217 Ile & 7.52 & 114.33 & 177.03 & - & 38.22 \\
217 Ile & - & - & - & 61.05 & - \\
221 Ile & - & - & 177.64 & 64.48 & 41.03 \\
222 Val & 6.84 & 116.18 & 176.29 & 65.31 & 29.92 \\
223 Ala & 8.43 & 125.24 & - & 51.6 & 19.97 \\
236 Thr & - & - & 178.78 & 66.34 & 68.31 \\
237 Ala & 8.28 & 119.98 & 179.67 & 55.24 & 17.01 \\
270 Gly & - & - & 175.75 & 44.9 & - \\
271 Gln & 7.87 & 121.19 & 177.35 & 54.83 & 30.87 \\
272 Ala & 8.89 & 124.02 & 179.66 & 55.57 & 17.4 \\
273 Glu & 8.32 & 114.87 & 178.91 & 57.47 & 29.88 \\
274 Tyr & 7.66 & 121.19 & 177.54 & 57.8 & 41.47 \\
275 Asp & 8.95 & 119.18 & 178.33 & 51.69 & 40.49 \\
297 Gly & - & - & 173.62 & 45.05 & - \\
298 Arg & 7.78 & 121.99 & 177.44 & 54.95 & 32.72 \\
& & & & &
\end{tabular}


Table A.6: List of the unassigned peaks in the hCANH spectrum of citrate-bound CitApc C12AR93A

\begin{tabular}{|c|c|c|c|}
\hline & ${ }^{1} \mathrm{H}[\mathrm{ppm}]$ & ${ }^{13} \mathrm{C}[\mathrm{ppm}]$ & ${ }^{15} \mathrm{~N}[\mathrm{ppm}]$ \\
\hline 1 & 10.54 & 54.50 & 129.82 \\
\hline 2 & 8.44 & 54.62 & 128.90 \\
\hline 3 & 9.47 & 42.34 & 125.61 \\
\hline 4 & 8.72 & 61.90 & 125.48 \\
\hline 5 & 8.84 & 57.33 & 123.59 \\
\hline 6 & 8.02 & 60.14 & 123.05 \\
\hline 7 & 8.12 & 57.91 & 122.98 \\
\hline 8 & 7.72 & 56.78 & 121.67 \\
\hline 9 & 7.76 & 54.71 & 121.19 \\
\hline 10 & 7.86 & 65.90 & 121.12 \\
\hline 11 & 7.81 & 56.37 & 120.64 \\
\hline 12 & 8.26 & 59.80 & 120.12 \\
\hline 13 & 8.00 & 57.81 & 119.96 \\
\hline 14 & 7.50 & 54.62 & 119.81 \\
\hline 15 & 7.78 & 59.65 & 119.48 \\
\hline 16 & 7.99 & 57.50 & 119.08 \\
\hline 17 & 7.68 & 67.18 & 118.34 \\
\hline 18 & 7.59 & 66.09 & 117.78 \\
\hline 19 & 6.61 & 60.30 & 117.55 \\
\hline 20 & 7.27 & 58.59 & 117.26 \\
\hline 21 & 7.81 & 61.68 & 117.19 \\
\hline 22 & 8.32 & 63.24 & 116.91 \\
\hline 23 & 7.99 & 58.44 & 116.54 \\
\hline 24 & 8.48 & 56.72 & 116.49 \\
\hline 25 & 7.95 & 57.47 & 116.44 \\
\hline 26 & 7.46 & 60.72 & 116.24 \\
\hline 27 & 7.82 & 62.82 & 115.03 \\
\hline 28 & 9.39 & 54.03 & 122.90 \\
\hline 29 & 8.06 & 60.70 & 120.75 \\
\hline 30 & 7.66 & 58.90 & 120.47 \\
\hline
\end{tabular}




\begin{tabular}{|c|c|c|c|}
\hline 31 & 7.91 & 54.27 & 119.72 \\
\hline 32 & 7.74 & 59.02 & 120.19 \\
\hline 33 & 8.61 & 55.23 & 121.22 \\
\hline 34 & 8.53 & 58.42 & 119.46 \\
\hline 35 & 7.90 & 59.49 & 116.66 \\
\hline 36 & 8.71 & 58.94 & 119.12 \\
\hline 37 & 7.46 & 59.46 & 118.60 \\
\hline 38 & 7.27 & 58.22 & 115.45 \\
\hline 39 & 8.50 & 58.67 & 117.52 \\
\hline 40 & 8.22 & 59.58 & 117.99 \\
\hline 41 & 8.91 & 59.06 & 114.94 \\
\hline 42 & 8.60 & 55.00 & 120.30 \\
\hline 43 & 7.78 & 58.82 & 118.27 \\
\hline 44 & 7.72 & 57.74 & 118.11 \\
\hline 45 & 8.00 & 57.80 & 118.02 \\
\hline 46 & 8.24 & 59.45 & 117.78 \\
\hline 47 & 7.83 & 58.41 & 117.65 \\
\hline 48 & 8.57 & 54.94 & 123.75 \\
\hline 49 & 9.08 & 50.96 & 123.74 \\
\hline 50 & 8.07 & 55.21 & 121.42 \\
\hline 51 & 7.87 & 57.98 & 120.18 \\
\hline 52 & 7.31 & 57.9 & 119.71 \\
\hline 53 & 8.34 & 57.93 & 118.32 \\
\hline 54 & 8.34 & 58.81 & 118.52 \\
\hline 55 & 8.15 & 57.68 & 117.46 \\
\hline 56 & 7.84 & 56.74 & 117.53 \\
\hline 57 & 8.14 & 58.58 & 117.71 \\
\hline 58 & 8.25 & 57.66 & 116.78 \\
\hline 59 & 8.17 & 58.54 & 116.51 \\
\hline 60 & 8.48 & 57.66 & 117.44 \\
\hline 61 & 6.93 & 59.25 & 115.25 \\
\hline 62 & 9.23 & 57.89 & 115.05 \\
\hline 63 & 8.25 & 53.43 & 116.92 \\
\hline
\end{tabular}




\begin{tabular}{|c|c|c|c|}
\hline 64 & 7.75 & 60.47 & 117.53 \\
\hline 65 & 8.09 & 61.42 & 117.37 \\
\hline 66 & 9.02 & 58.92 & 117.52 \\
\hline 67 & 8.81 & 57.42 & 117.43 \\
\hline 68 & 7.78 & 61.84 & 118.83 \\
\hline 69 & 8.66 & 58.10 & 116.79 \\
\hline 70 & 8.69 & 56.96 & 117.25 \\
\hline 71 & 7.74 & 58.21 & 116.65 \\
\hline 72 & 7.40 & 64.04 & 111.70 \\
\hline 73 & 8.33 & 47.52 & 107.66 \\
\hline 74 & 8.24 & 55.24 & 119.90 \\
\hline 75 & 8.53 & 60.29 & 120.23 \\
\hline 76 & 8.40 & 57.91 & 120.99 \\
\hline 77 & 7.70 & 60.22 & 120.46 \\
\hline 78 & 8.47 & 55.70 & 119.90 \\
\hline 79 & 9.19 & 55.65 & 123.54 \\
\hline 80 & 8.90 & 53.05 & 123.08 \\
\hline 81 & 8.87 & 55.25 & 121.88 \\
\hline 82 & 7.99 & 54.54 & 121.05 \\
\hline 83 & 8.69 & 58.61 & 118.84 \\
\hline 84 & 8.06 & 57.22 & 116.17 \\
\hline 85 & 8.15 & 57.36 & 115.34 \\
\hline 86 & 9.25 & 60.30 & 128.93 \\
\hline 87 & 8.32 & 65.61 & 120.31 \\
\hline 88 & 9.87 & 44.34 & 123.46 \\
\hline 89 & 7.92 & 64.80 & 121.62 \\
\hline 90 & 7.73 & 45.27 & 107.55 \\
\hline 91 & 8.64 & 59.08 & 121.83 \\
\hline 92 & 8.70 & 57.51 & 116.31 \\
\hline 93 & 8.43 & 47.56 & 107.39 \\
\hline 94 & 7.76 & 60.41 & 118.58 \\
\hline 95 & 7.77 & 60.38 & 118.49 \\
\hline 96 & 8.92 & 69.15 & 111.82 \\
\hline
\end{tabular}




\begin{tabular}{l|l|l|l}
97 & 9.14 & 54.42 & 124.70 \\
98 & 9.28 & 54.94 & 121.06 \\
99 & 8.94 & 58.93 & 118.38 \\
100 & 8.29 & 58.05 & 120.28 \\
101 & 8.35 & 57.89 & 120.22 \\
102 & 7.66 & 54.73 & 120.37
\end{tabular}

\section{A.3 Pulse programs}

\section{A.3.1 Liquid-state NMR}

\section{${ }^{15} \mathrm{~N}-\mathrm{HSQC}$}

;hsqc15N.new

;D. Lee, Nov. 2002

;15N-1H HSQC correlations without water saturation

;The delay for 3-9-19 watergate (d5) should be matched ; with $1 / \mathrm{d}$; d=distance of next null point (in $\mathrm{Hz}$ ).

;S. Mori et al, JMR B108, 94-98 (1995)

;pl1 : power for $1 \mathrm{H}$

;pl2 : power for $13 \mathrm{C}$

;pl3 : power for $15 \mathrm{~N}$

;pl13 : power for 15N waltz16 decoupling

;p1 : 90 degree hard pulse $1 \mathrm{H}$

;p3 : 90 degree hard pulse 13C

;p4 : 180 degree hard 13C pulse (225d for 5/600)

;p5 : 90 degree hard pulse $15 \mathrm{~N}$

;pcpd3 : 90 deg cpd-pulse15N(waltz16,160u)

;d1 : relaxation delay

;d2 : INEPT delay $(2.7 \mathrm{~m})$

;d5 : delay for $3-9-19=1 /(\mathrm{Hz}$ between nulls $)$

;in0 : 1/(2 SW) (Hz) 
;p21 : 500u (Gradient in first INEPT)

;p22 : 500u (Gradient for z-filter)

;p23 : 1m (Gradient for second INEPT)

;gpz1 : 19\%

;gpz2 : $30 \%$

;gpz3 : 65\%

\# include ¡Avance.incli

define delay INEPT_W

define delay INEPT_D

define GRADIENT1 10u p21:gp1 200u

define GRADIENT2 10u p22:gp2 200u

define GRADIENT3 10u p23:gp3 200u

$" \mathrm{p} 2=2^{*} \mathrm{p} 1 "$

$" \mathrm{p} 6=2 * \mathrm{p} 5 "$

"in0=inf1/2"

"d0=in0/2-p5*2/3.14159-p1"

"d $3=\mathrm{d} 5 / 2-\mathrm{p} 5 "$

"INEPT_D=d2-p21-210u"

"INEPT_W=d2-(p23+210u+p1*2.3846+d5*2.5)"

$110 u$ ze

$21 \mathrm{~m}$ do:f3

d1 pl1:f1

20u pl3:f3

20u LOCKH_ON

; first INEPT

(p1 ph20):f1

GRADIENT1

INEPT_D

(center(p2 ph21):f1 (p6 ph1):f3)

GRADIENT1

INEPT_D

(p1 ph21):f1

GRADIENT2 
$;-15 \mathrm{~N}$ evolution

(p5 ph1):f3

(refalign (d0 p2 ph23 d0):f1 center (p3 ph23 1.5u p4 ph20 1.5u p3 ph23):f2)

(p5 ph20):f3

GRADIENT2

; second INEPT

(p1 ph22):f1

GRADIENT3

INEPT_W

(p1*0.2308 ph21 d5 p1*0.6923 ph21 d5 p1*1.4615 ph21):f1

(d3 p6 ph1 d3):f3

(p1*1.4615 ph23 d5 p1*0.6923 ph23 d5 p1*0.2308 ph23):f1

GRADIENT3

INEPT_W pl13:f3 LOCKH_OFF

;-acquisition

go $=2 \operatorname{ph} 31 \operatorname{cpd} 3: \mathrm{f} 3$

$1 \mathrm{~m}$ do:f3 mc 0 to 2 F1PH(ip1,id0)

10u do:f1

10u do:f2

10u do:f3

;10u do:f4

10u LOCKH_OFF

exit

ph1 $=02$

ph $31=20$

ph20 $=0$

ph21 $=1$

ph $22=2$

$\operatorname{ph} 23=3$

\section{${ }^{15}$ N-NOESY-HSQC}

;hsqc15N.new

;D. Lee, Nov. 2002

;15N-1H HSQC correlations without water saturation 
;The delay for 3-9-19 watergate (d5) should be matched ; with $1 / \mathrm{d} ; \mathrm{d}=$ distance of next null point (in $\mathrm{Hz}$ ).

;S. Mori et al, JMR B108, 94-98 (1995)

;pl1 : power for $1 \mathrm{H}$

;pl2 : power for 13C

;pl3 : power for $15 \mathrm{~N}$

;pl13 : power for $15 \mathrm{~N}$ waltz16 decoupling

;p1 : 90 degree hard pulse 1H

;p3 : 90 degree hard pulse 13C

;p4 : 180 degree hard 13C pulse (225d for 5/600)

;p5 : 90 degree hard pulse $15 \mathrm{~N}$

;pcpd3 : 90 deg cpd-pulse15N(waltz16,160u)

;d1 : relaxation delay

;d2 : INEPT delay ( $2.7 \mathrm{~m})$

;d5 : delay for $3-9-19=1 /(\mathrm{Hz}$ between nulls $)$

d8 : NOESY mixing

;in0 : 1/(2 SW) $(\mathrm{Hz})$

;p20 : 1m (Gradient in cleaning)

;p21 : 500u (Gradient in first INEPT)

;p22 : 500u (Gradient for z-filter)

;p23 : 1m (Gradient for second INEPT)

;p24 : 500u (Gradient for z-filter)

;p25 : 1m (Gradient for mixing)

;gpz0 : 80\%

;gpz1 : $19 \%$

;gpz2 : $30 \%$

;gpz3 : 65\%

;gpz4 : $37 \%$

;gpz5 : 60\%

;cnst22: $13 \mathrm{C}$ betw. $\mathrm{CO}$ and $\mathrm{C}($ ali) [100ppm]

;cnst23: 13C betw. C(ali) and C(aro) [70ppm]

include ¡Avance_dl.incli

define delay INEPT_W

define delay INEPT_D 
define GRADIENT0 10u p20:gp0 200u

define GRADIENT1 10u p21:gp1 200u

define GRADIENT2 10u p22:gp2 200u

define GRADIENT3 10u p23:gp3 200u

define GRADIENT4 10u p24:gp4 200u

define GRADIENT5 10u p25:gp5 200u

$" \mathrm{p} 2=2^{*} \mathrm{p} 1 "$

$" \mathrm{p} 6=2^{*} \mathrm{p} 5 "$

"in0=inf1/2"

"in10=inf2/2"

"d0=in0/2-p1*2/3.14159"

"d10=in10/2-p5*2/3.14159"

$" \mathrm{~d} 7=\mathrm{d} 8-(\mathrm{p} 25+210 \mathrm{u}) "$

"d $3=\mathrm{d} 5 / 2-\mathrm{p} 5 "$

"INEPT_D=d2-p21-210u"

"INEPT_W=d2- $(\mathrm{p} 23+210 \mathrm{u}+\mathrm{p} 1 * 2.3846+\mathrm{d} 5 * 2.5) "$

aqseq 312

$110 u$ ze

$21 \mathrm{~m}$

10u do:f3

d1

10u pl1:f1

10u pl2:f2

20u pl3:f3

$20 \mathrm{u}$ LOCKH_ON

$10 \mathrm{u}$

GRADIENT0

$4 \mathrm{~m}$

$10 \mathrm{u} \mathrm{fq}=\mathrm{cnst} 23(\mathrm{bf} \mathrm{ppm}): \mathrm{f} 2$

$20 \mathrm{u}$

(refalign (p1 ph1 d0 d0 p1 ph2):f1 center (p6 ph20):f3 center(p3 ph21 1.5u p4 ph20 1.5u p3 ph21):f2)

GRADIENT5

$\mathrm{d} 7 \mathrm{fq}=\mathrm{cnst} 22(\mathrm{bf} \mathrm{ppm}): \mathrm{f} 2$

; first INEPT 
(p1 ph20):f1

GRADIENT1

INEPT_D

(center(p2 ph21):f1 (p6 ph20):f3)

GRADIENT1

INEPT_D

(p1 ph21):f1

GRADIENT2

; $15 \mathrm{~N}$ evolution

; (p5 ph3):f3

(refalign (p5 ph3 d10 d10 p5 ph20):f3 center (p2 ph23):f1 center (p3 ph23 1.5u p4 ph20 1.5u p3 ph23):f2)

; (p5 ph20):f3

GRADIENT4

; second INEPT

(p1 ph22):f1

GRADIENT3

INEPT_W

(p1*0.2308 ph21 d5 p1*0.6923 ph21 d5 p1*1.4615 ph21):f1

(d3 p6 ph20 d3):f3

(p1*1.4615 ph23 d5 p1*0.6923 ph23 d5 p1*0.2308 ph23):f1

GRADIENT3

INEPT_W pl13:f3 LOCKH_OFF

; acquisition

go $=2 \operatorname{ph} 31 \operatorname{cpd} 3: f 3$

$1 \mathrm{~m}$ do:f3 mc \#0 to 2

F1PH(ip1,id0)

F2PH(rp1 \& rd0 \& ip3,id10)

10u do:f1

10u do:f2

10u do:f3

10u LOCKH_OFF

exit

ph1 $=0022$

ph2 $=00002222$

ph3 $=02$ 
ph31=2 0020220

ph20 $=0$

ph21 $=1$

$\operatorname{ph} 22=2$

$\operatorname{ph} 23=3$

\section{${ }^{13}$ C-NOESY-HSQC}

Bruker standard pulse program: noesyhsqcetgp3d

\section{HCCH-TOCSY}

Bruker standard pulse program: hcchdigp3d2

\section{HNCA}

Bruker standard pulse program: hncagpwg3d

\section{CBCACONH}

Bruker standard pulse program: cbcaconhgpwg3d

\section{HNCO}

Bruker standard pulse program: hncogp3d

\section{A.3.2 ${ }^{13} \mathrm{C}$ detected ssNMR}

\section{hNCA}

Bruker standard pulse program: hNCa.dcp

\section{hNCACB}

Bruker standard pulse program: hNCaCx3D.dcp

\section{A.3.3 ${ }^{1} \mathbf{H}$ detected ssNMR}

\section{${ }^{1} \mathrm{H}$-detected ${ }^{15} \mathrm{~N}-{ }^{1} \mathrm{H} 2 \mathrm{D}$ correlation}


The pulse sequence for the ${ }^{1} \mathrm{H}$-detected ${ }^{15} \mathrm{~N}-{ }^{1} \mathrm{H} 2 \mathrm{D}$ correlation $(\mathrm{hNH})$ is reported in figure A.1, with an illustration of the coherence transfers and spins involved Barbet-Massin et al. (2014). Orange boxes indicate cross-polarization, and gray boxes indicate heteronuclear decoupling. The MISSISSIPPI sequence (Zhou and Rienstra, 2008) is used as water suppression block and it is indicated by the striped box. All pulses are of phases 0 , unless indicated differently. $\phi 1=13, \phi 2=1, \phi 3=002$ $2, \phi 4=1, \phi 5=1 * 43 * 4, \phi$ rec $=13313113$.

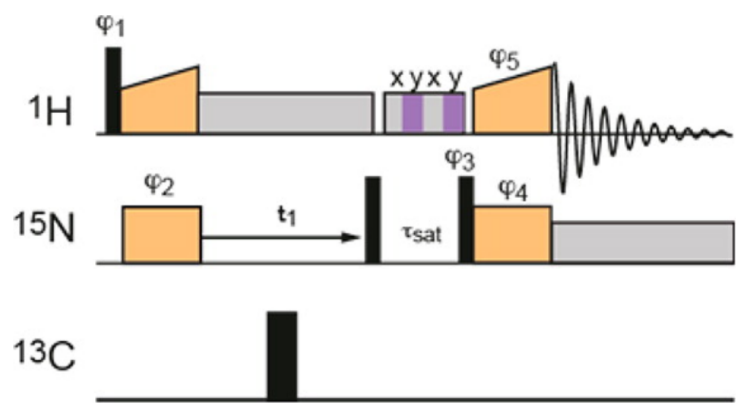

Figure A.1: Pulse sequence for the ${ }^{1} \mathrm{H}$-detected ${ }^{15} \mathrm{~N}-{ }^{1} \mathrm{H} 2 \mathrm{D}$ correlation (hNH).

\section{${ }^{13} \mathrm{C}-{ }^{15} \mathrm{~N}-{ }^{1} \mathrm{H}$ 3D correlation experiments}

The pulse sequences for the six ${ }^{1} \mathrm{H}$-detected ${ }^{13} \mathrm{C}-{ }^{15} \mathrm{~N}-{ }^{1} \mathrm{H} 3 \mathrm{D}$ correlation experiments are reported in figures A.2,A.3 and A.4 with an illustration of the coherence transfers and spins involved (Barbet-Massin et al., 2014). Narrow and broad black rectangles indicate $\pi / 2$ and $\pi$ pulses, respectively, and bell shapes represent selective inversion pulses. Orange boxes indicate cross-polarization, and gray boxes indicate heteronuclear decoupling. The MISSISSIPPI sequence (Zhou and Rienstra, 2008) is used as water suppression block and it is indicated by the striped box. Spin-echoes involved in the transfer between $\mathrm{CO}$ and $\mathrm{CA}$ are highlighted in blue and red, when the coherence is present on the $\mathrm{CO}$ and $\mathrm{CA}$, respectively, and spin-echoes involved in transfer between $\mathrm{CA}$ and $\mathrm{CB}$ are highlighted in green. All pulses are of phases 0 , unless indicated differently. (hNCA in figure A.2 and hNCO in figure A.3) $\phi 1=02, \phi 20=1, \phi 2=1, \phi 6=0022, \phi 12=1, \phi 7=1, \phi 11=1^{*} 4$ $3^{*} 4, \phi$ rec $=13313113$; (b) $\phi 1=02, \phi 20=1, \phi 2=1, \phi 6=0022, \phi 12=1, \phi 7=1, \phi 11$ $=1, \phi 14=0 * 41 * 4, \phi 17=0 * 81 * 8, \phi$ rec $=3113133113313$ 113; (hcoCAcoNH in figure A.2 and hcaCBcaNH in figure A.4 ) $\phi 1=13, \phi 2=1$ 133, $\phi 4=1, \phi 5=1, \phi 7$ $=1 * 43 * 4, \phi 8=0 * 82 * 8, \phi 9=3, \phi 10=1, \phi$ rec $=0220200220020220 ;$ (f) $\phi 1=13$, $\phi 2=1133, \phi 4=1, \phi 5=1, \phi 6=0+162^{*} 16, \phi 9=3, \phi 10=1, \phi 14=0 * 81 * 8, \phi 16=$ $3, \phi 17=0 * 41^{*} 4, \phi$ rec $=02202002200202202002022002202$ 


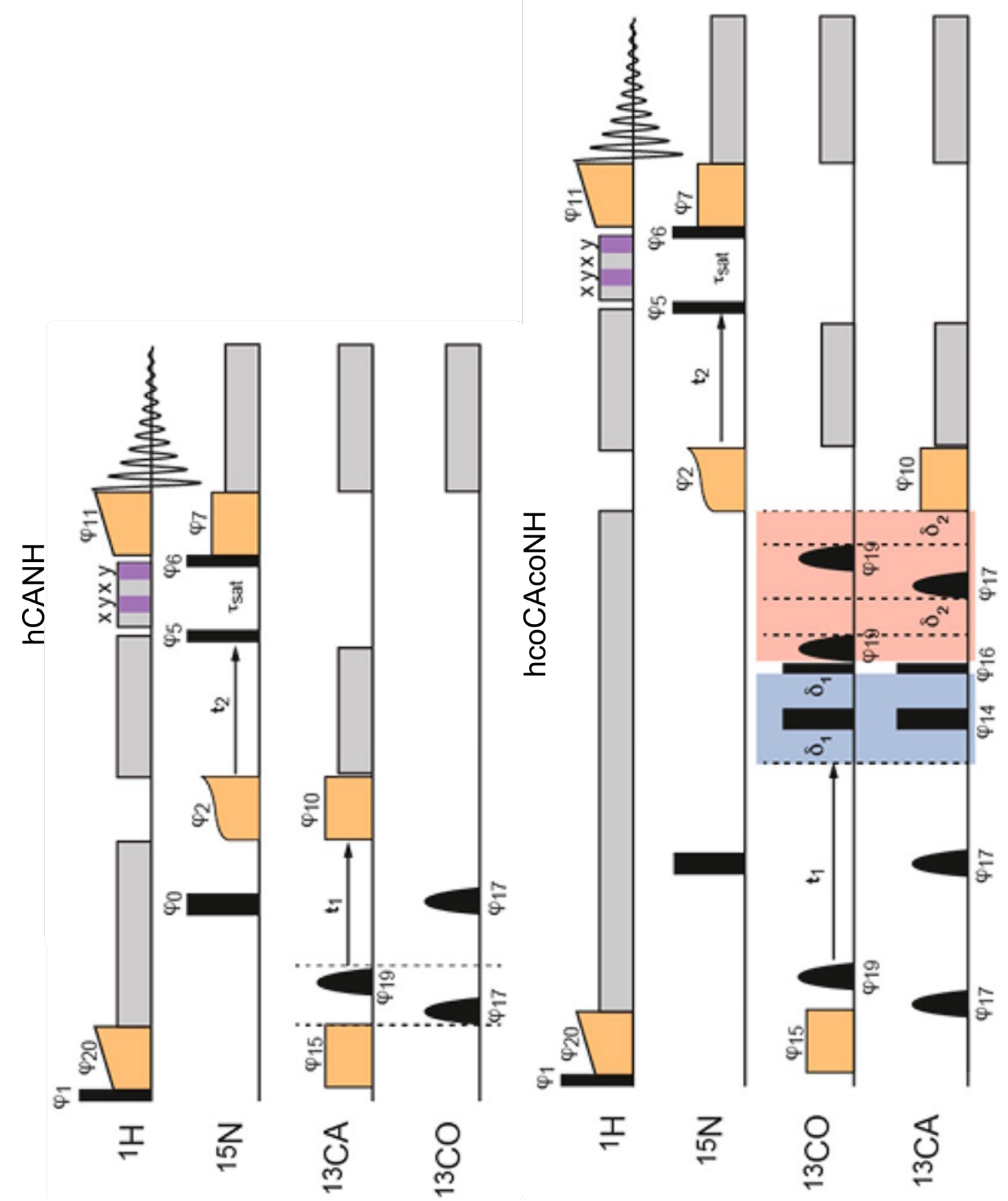

Figure A.2: Pulse sequences for the intraresidue hCANH experiment (left) and inter-residue hcoCAcoNH experiment, with an illustration of the coherence transfers and spins involved.

00 2. Specific $\mathrm{CP}$ to or from ${ }^{13} \mathrm{C}$ may require a frequency shift, and in this case, the phases of rf during the two ${ }^{13} \mathrm{C} \mathrm{CP}$ periods are aligned respectively at the end and at the beginning of the contact time. 


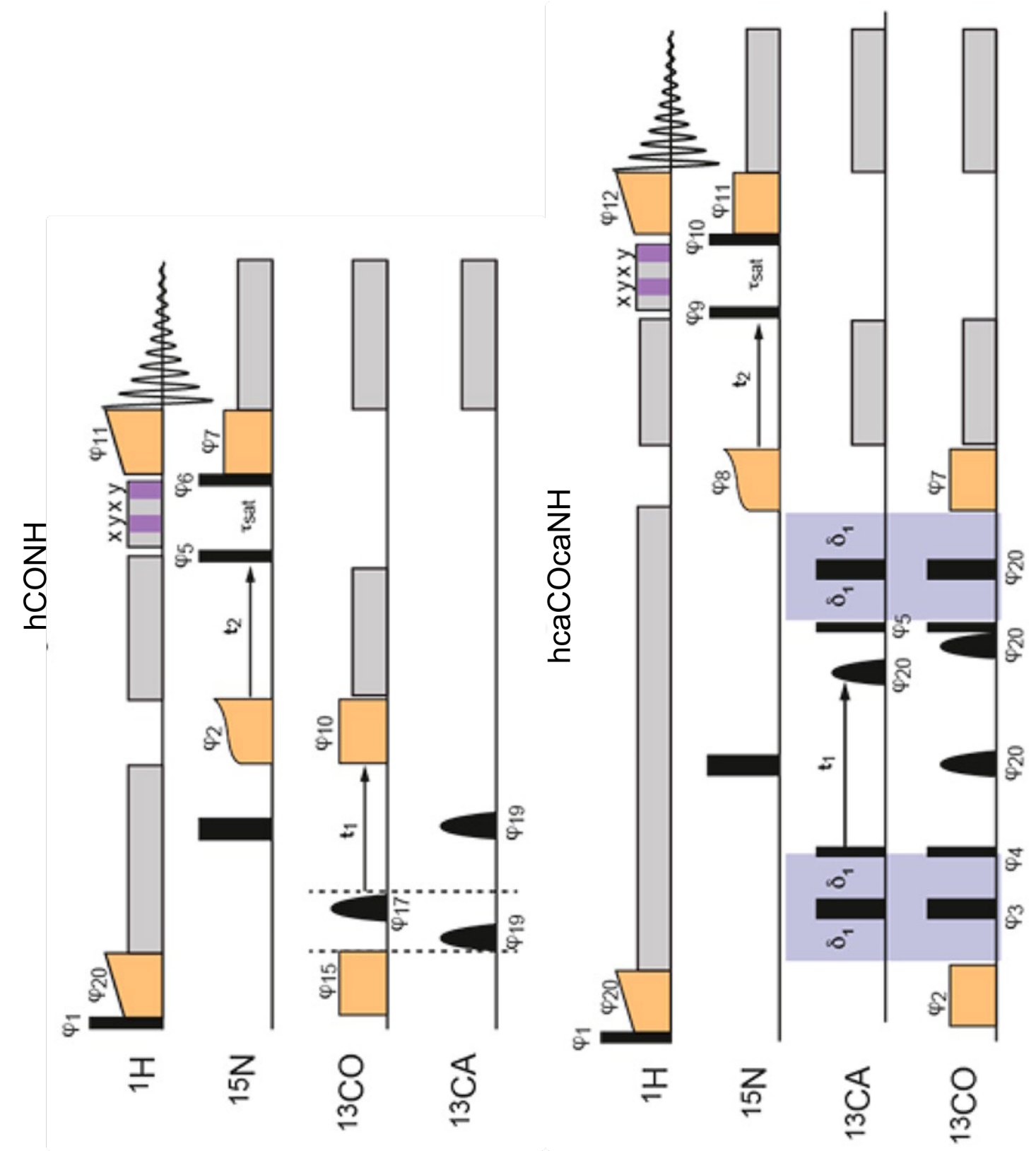

Figure A.3: Pulse sequences for the intraresidue hCONH experiment (left) and inter-residue hcaCOcaNH experiment, with an illustration of the coherence transfers and spins involved. 

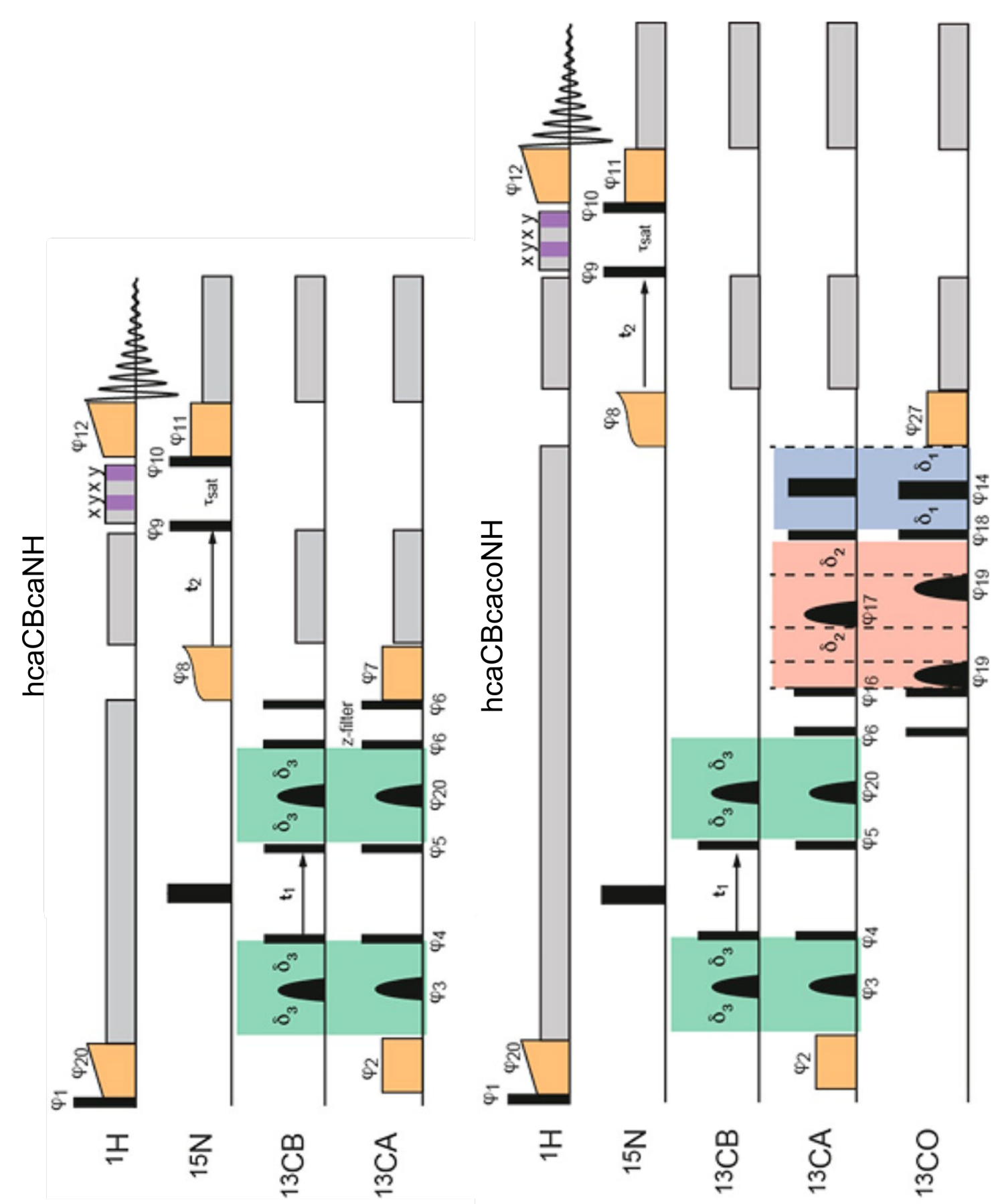

Figure A.4: Pulse sequences for the intraresidue hcaCBcaNH experiment (left) and inter-residue hcaCBcacoNH experiment, with an illustration of the coherence transfers and spins involved. 


\section{Bibliography}

L. A. Abriata, D. Albanesi, M. D. Peraro, and D. D. Mendoza. Signal Sensing and Transduction by Histidine Kinases as Unveiled through Studies on a Temperature Sensor. Accounts of chemical research, 50:1359-1366, 2017. doi: 10.1021/acs.accounts.6b00593.

C. A. Adase, R. R. Draheim, and M. D. Manson. The Residue Composition of the Aromatic Anchor of the Second Transmembrane Helix Determines the Signaling Properties of the Aspartate/Maltose Chemoreceptor Tar of Escherichia coli. Biochemistry, 51:1925-1932, 2012. doi: 10.1021/bi201555x.

M. V. Airola, K. J. Watts, A. M. Bilwes, and B. R. Crane. Article Structure of Concatenated HAMP Domains Provides a Mechanism for Signal Transduction. Structure, 18(4):436-448, 2010. ISSN 0969-2126. doi: 10.1016/j.str.2010.01.013. URL http://dx.doi.org/10.1016/j.str.2010.01.013.

D. Albanesi, M. Martin, F. Trajtenberg, M. C. Mansilla, A. Haouz, P. M. Alzari, D. de Mendoza, and A. Buschiazzo. Structural plasticity and catalysis regulation of a thermosensor histidine kinase. Proceedings of the National Academy of Sciences, 106(38):16185-16190, 2009. ISSN 0027-8424. doi: 10. 1073/pnas.0906699106. URL http://www.pnas.org/cgi/doi/10.1073/pnas. 0906699106.

S. F. Altschul, W. Gish, W. Miller, E. W. Myers, and D. J. Lipman. Basic local alignment search tool. Journal of molecular biology, 215(3):403-10, 1990. ISSN 0022-2836. doi: 10.1016/S0022-2836(05)80360-2. URL http: //www.sciencedirect.com/science/article/pii/S0022283605803602.

O. Ashenberg, A. E. Keating, and M. T. Laub. Helix bundle loops determine whether histidine kinases autophosphorylate in cis or in trans. Journal of Molecular Biology, 425(7):1198-1209, 2013. ISSN 00222836. doi: 10.1016/j.jmb.2013. 01.011. URL http://dx.doi.org/10.1016/j.jmb.2013.01.011.

L. Banci, I. Bertini, and C. Luchinat. Nuclear and electron relaxation: the magnetic nucleus-unpaired electron coupling in solution. Wiley-VCH, 1991.

E. Barbet-Massin, A. J. Pell, J. S. Retel, L. B. Andreas, K. Jaudzems, W. T. Franks, A. J. Nieuwkoop, M. Hiller, V. Higman, P. Guerry, A. Bertarello, M. J. 
Knight, M. Felletti, T. Le Marchand, S. Kotelovica, I. Akopjana, K. Tars, M. Stoppini, V. Bellotti, M. Bolognesi, S. Ricagno, J. J. Chou, R. G. Griffin, H. Oschkinat, A. Lesage, L. Emsley, T. Herrmann, and G. Pintacuda. Rapid proton-detected NMR assignment for proteins with fast magic angle spinning. Journal of the American Chemical Society, 136(35):12489-97, sep 2014. ISSN 1520-5126. doi: 10.1021/ja507382j. URL http://pubs.acs.org/doi/10. 1021/ja507382jhttp://www .ncbi.nlm.nih.gov/pubmed/25102442http: //www . pubmedcentral .nih.gov/articlerender. fcgi?artid=PMC4156866.

A. Bernini, V. Venditti, O. Spiga, A. Ciutti, F. Prischi, R. Consonni, L. Zetta, I. Arosio, P. Fusi, A. Guagliardi, and N. Niccolai. Biophysical Chemistry NMR studies on the surface accessibility of the archaeal protein Sso7d by using TEMPOL and Gd ( III )( DTPA-BMA ) as paramagnetic probes. Biophysical Chemistry, 137:71-75, 2008. doi: 10.1016/j.bpc.2008.07.003.

M. A. Bhate, K. A. Molnar, M. Goulian, and W. F. Degrado. Signal Transduction in Histidine Kinases: Insights from New Structures. Structure, 23(6):981-996, 2015. ISSN 18784186. doi: 10.1016/j.str.2015.04.002. URL http://dx.doi. org/10.1016/j.str.2015.04.002.

A. Bhattacharya, R. Tejero, and G. T. Montelione. Evaluating protein structures determined by structural genomics consortia. Proteins: Structure, Function, and Bioinformatics, 66(4):778-795, 2007.

M. Bott, M. Meyer, and P. Dimroth. Regulation of anaerobic citrate metabolism in Klebsiella pneumoniae. 18:533-546, 1995.

P. Boyer, M. DeLuca, K. Ebner, D. Hultquist, and J. Peter. Identification of Phosphohistidine Digests from a Probable Intermediate of Qxidative Phosphorylation. Journal of Biological Chemistry, 237(10):PC3306 - PC3308, 1962. ISSN 0021-9258.

D. Bray. Protein molecules as computational elements in living cells. Nature, 376 (6538):307-312, 1995. ISSN 00280836. doi: 10.1038/376307a0.

P. Casino, V. Rubio, and A. Marina. Structural Insight into Partner Specificity and Phosphoryl Transfer in Two-Component Signal Transduction. Cell, 139(2): 325-336, 2009. ISSN 00928674. doi: 10.1016/j.cell.2009.08.032. URL http: //dx.doi.org/10.1016/j.cell.2009.08.032. 
P. Casino, L. Miguel-Romero, and A. Marina. Visualizing autophosphorylation in histidine kinases. Nature Communications, 5:1-11, 2014. ISSN 20411723. doi: 10.1038/ncomms4258. URL http://dx.doi.org/10.1038/ncomms4258.

V. Cherozov. Lipidic Cubic Phase Technologies for Membrane Protein Structural Studies. Current Opinion in Structural Biology, 21(4):559-566, 2011. doi: 10. 1016/j.sbi.2011.06.007.Lipidic.

J. Cheung and W. A. Hendrickson. Crystal Structures of C 4 -Dicarboxylate Ligand Complexes with Sensor Domains of Histidine Kinases DcuS and DctB. 283(44):30256-30265, 2008. doi: 10.1074/jbc.M805253200.

J. Cheung and W. A. Hendrickson. Structural Analysis of Ligand Stimulation of the Histidine Kinase NarX. Structure, 17(2):190-201, 2009. ISSN 09692126. doi: 10.1016/j.str.2008.12.013. URL http://dx.doi.org/10.1016/ j.str.2008.12.013.

J. Cheung, C. A. Bingman, M. Reyngold, W. A. Hendrickson, and C. D. Waldburger. Crystal structure of a functional dimer of the PhoQ sensor domain. Journal of Biological Chemistry, 283(20):13762-13770, 2008. ISSN 00219258. doi: $10.1074 /$ jbc.M710592200.

U. S. Cho, M. W. Bader, M. F. Amaya, M. E. Daley, R. E. Klevit, S. I. Miller, and W. Xu. Metal bridges between the PhoQ sensor domain and the membrane regulate transmembrane signaling. Journal of Molecular Biology, 356(5):11931206, 2006. ISSN 00222836. doi: 10.1016/j.jmb.2005.12.032.

P. J. A. Cock and D. E. Whitworth. Evolution of Prokaryotic Two-Component System Signaling Pathways : Gene Fusions and Fissions. Molecular Biology and Evoulution, 24(11):2355-2357, 2007. doi: 10.1093/molbev/msm170.

L. E. Cybulski, D. Albanesi, M. C. Mansilla, S. Altabe, P. S. Aguilar, and D. de Mendoza. Mechanism of membrane fluidity optimization: isothermal control of the $<\mathrm{i}>$ Bacillus subtilis $</ \mathrm{i}>$ acyl-lipid desaturase. Mol. Microbiol., 45:1379-1388, 2002.

F. Delaglio, S. Grzesiek, G. W. Vuister, G. Zhu, J. Pfeifer, and A. D. Bax. NMRPipe: a multidimensional spectral processing system based on UNIX pipes. Journal of biomolecular NMR, 6(3):277-293, 1995. 
R. R. Draheim, A. F. Bormans, R.-z. Lai, and M. D. Manson. Tuning a Bacterial Chemoreceptor with Protein - Membrane Interactions. Biochemistry, 45:1465514664, 2006. doi: 10.1021/bi061259i.

M. Etzkorn, H. Kneuper, P. Dünnwald, V. Vijayan, J. Krämer, C. Griesinger, S. Becker, G. Unden, and M. Baldus. Plasticity of the PAS domain and a potential role for signal transduction in the histidine kinase DcuS. Nature Structural Ef Molecular Biology, 15(10):1031-1039, 2008. doi: 10.1038/nsmb.1493.

L. Feng, W. Wang, J. Cheng, Y. Ren, G. Zhao, C. Gao, Y. Tang, X. Liu, W. Han, $\mathrm{X}$. Peng, and Others. Genome and proteome of long-chain alkane degrading Geobacillus thermodenitrificans NG80-2 isolated from a deep-subsurface oil reservoir. Proceedings of the National Academy of Sciences, 104(13):5602-5607, 2007.

P. Fricke, V. Chevelkov, M. Zinke, K. Giller, S. Becker, and A. Lange. Backbone assignment of perdeuterated proteins by solid-state NMR using proton detection and ultrafast magic-Angle spinning. Nature Protocols, 12(4): 764-782, 2017. ISSN 17502799. doi: 10.1038/nprot.2016.190. URL http: //dx.doi.org/10.1038/nprot.2016.190.

R. Gao and A. M. Stock. Biological Insights from Structures of TwoComponent Proteins. Annual Review of Microbiology, 63(1):133-154, 2009. ISSN 0066-4227. doi: 10.1146/annurev.micro.091208.073214. URL http: //www . annualreviews .org/doi/10.1146/annurev. micro.091208.073214.

T. Gerharz, S. Reinelt, S. Kaspar, L. Scapozza, and M. Bott. Identification of basic amino acid residues important for citrate binding by the periplasmic receptor domain of the sensor kinase CitA. Biochemistry, 42(19):5917-5924, 2003. ISSN 00062960. doi: 10.1021/bi0340595.

P. Golby, S. Davies, D. J. Kelly, J. R. Guest, and S. C. Andrews. Identification and Characterization of a Two-Component Sensor-Kinase and ResponseRegulator System ( DcuS-DcuR ) Controlling Gene Expression in Response to C 4 -Dicarboxylates in Escherichia coli. 181(4):1238-1248, 1999.

W. Gong, B. Hao, and M. K. Chan. New mechanistic insights from structural studies of the oxygen-sensing domain of Bradyrhizobium japonicum FixL. Biochemistry, 39(14):3955-3962, 2000. ISSN 00062960. doi: 10.1021/bi992346w. 
I. Gushchin and V. Gordeliy. Transmembrane Signal Transduction in TwoComponent Systems: Piston, Scissoring, or Helical Rotation? BioEssays, 40 (2):1-10, 2018. ISSN 15211878. doi: 10.1002/bies.201700197.

I. Gushchin, V. Gordeliy, and S. Grudinin. Two Distinct States of the HAMP Domain from Sensory Rhodopsin Transducer Observed in Unbiased Molecular Dynamics Simulations. PloS ONE, 8(7):1-8, 2013. doi: 10.1371/journal.pone. 0066917.

I. Gushchin, I. Melnikov, V. Polovinkin, A. Ishchenko, A. Yuzhakova, P. Buslaev, G. Bourenkov, S. Grudinin, E. Round, T. Balandin, V. Borshchevskiy, D. Willbold, G. Leonard, G. Büldt, A. Popov, and V. Gordeliy. Mechanism of transmembrane signaling by sensor histidine kinases. Science, 356(6342), 2017. ISSN 10959203. doi: 10.1126/science.aah6345.

P. I. Hanson and H. Schulman. NEURONAL Ca2+/CALMODULINDEPENDENT PROTEIN KINASES. Ann. Rev. Biochem., 61:559-601, 1992. ISSN 0066-4154. doi: 10.1146/annurev.bi.61.070192.003015. URL http://www . ncbi.nlm.nih.gov/pubmed/1323238.

C. Hartlmüller, C. Göbl, and T. Madl. Prediction of protein structure using surface accessibility data. Angewandte Chemie International Edition, 55(39): 11970-11974, 2016.

C. Hartlmüller, J. C. Gün, A. C. Wolter, and J. Wöhn. RNA structure refinement using NMR solvent accessibility data. Scientific Reports, 7(March):1-10, 2017. doi: 10.1038/s41598-017-05821-z.

G. L. Hazelbauer, J. J. Falke, and J. S. Parkinson. Bacterial chemoreceptors : highperformance signaling in networked arrays. Trends in Biochemical Sciences, 33 (1):9-19, 2007. doi: 10.1016/j.tibs.2007.09.014.

J. F. Hess, K. Oosawa, P. Matsumura, and M. I. Simon. Protein phosphorylation is involved in bacterial chemotaxis. Proceedings of the National Academy of Sciences, 84(21):7609-7613, 1987. ISSN 0027-8424. doi: 10.1073/pnas.84.21. 7609. URL http://www.pnas.org/cgi/doi/10.1073/pnas.84.21.7609.

a. G. Hughson and G. L. Hazelbauer. Detecting the conformational change of transmembrane signaling in a bacterial chemoreceptor by measuring effects on disulfide cross-linking in vivo. Proceedings of the National Academy of Sciences 
of the United States of America, 93(21):11546-11551, 1996. ISSN 00278424. doi: 10.1073/pnas.93.21.11546.

M. Hulko, F. Berndt, M. Gruber, J. U. Linder, V. Truffault, A. Schultz, J. Martin, J. E. Schultz, A. N. Lupas, and M. Coles. The HAMP Domain Structure Implies Helix Rotation in Transmembrane Signaling. Cell, 126(5):929-940, 2006. ISSN 00928674. doi: 10.1016/j.cell.2006.06.058.

M. Hulko, K. Hantke, H. U. Ferris, S. Dunin-horkawicz, L. Garc1, J. E. Schultz, K. Zeth, A. N. Lupas, and M. Coles. The Mechanisms of HAMP-Mediated Signaling in Transmembrane Receptors. pages 378-385, 2011. doi: 10.1016/j. str.2011.01.006.

D. E. Hultquist. The preparation and characterization of phosphorylated derivatives of histidine. BBA - Bioenergetics, 153(2):329-340, 1968. ISSN 00052728. doi: 10.1016/0005-2728(68)90078-9.

D. E. Hultquist, R. W. Moyer, and P. D. Boyer. The Preparation and Characterization of 1-Phosphohistidine and 3-Phosphohistidine. Biochemistry, 5(1): 322-331, 1966. ISSN 15204995. doi: 10.1021/bi00865a041.

S. Kaspar and M. Bott. The sensor kinase CitA (DpiB) of Escherichia coli functions as a high-affinity citrate receptor. Archives of Microbiology, 177(4):313-321, apr 2002. ISSN 1432-072X. doi: 10.1007/s00203-001-0393-z. URL https: //doi.org/10.1007/s00203-001-0393-z.

S. Kaspar, R. Perozzo, S. Reinelt, M. Meyer, K. Pfister, L. Scapozza, and M. Bott. The periplasmic domain of the histidine autokinase CitA functions as a highly specific citrate receptor. Molecular Microbiology, 33(4):858-872, 1999. ISSN 0950382X. doi: 10.1046/j.1365-2958.1999.01536.x.

L. E. Kay, G. M. Clore, A. Bax, and A. M. Gronenborn. Four-dimensional heteronuclear triple-resonance NMR spectroscopy of interleukin-1 beta in solution. Science, 249(4967):411 LP - 414, jul 1990. URL http://science. sciencemag. org/content/249/4967/411. abstract.

I. Kazuya, S. Nagasawa, S.-i. Tokishita, and M. Takeschi. A novel device of bacterial signal transducers. The EMBO journal, 13(21):5195-5202, 1994. 
J. Key, M. Hefti, E. B. Purcell, and K. Moffat. Structure of the redox sensor domain of Azotobacter vinelandii NifL at atomic resolution: Signaling, dimerization, and mechanism. Biochemistry, 46(12):3614-3623, 2007. ISSN 00062960. doi: 10.1021/bi0620407.

E. C. Kofoid and J. S. Parkinson. Transmitter and receiver modules in bacterial signaling proteins. Proceedings of the National Academy of Sciences of the United States of America, 85(July):4981-4985, 1988.

J. Lacal, A. Busch, M.-E. Guazzaroni, T. Krell, and J. L. Ramos. The TodS-TodT two-component regulatory system recognizes a wide range of effectors and works with DNA-bending proteins. Proceedings of the National Academy of Sciences, 103(21):8191-8196, 2006. ISSN 0027-8424. doi: 10.1073/pnas.0602902103. URL http://www . pnas.org/cgi/doi/10.1073/pnas. 0602902103.

J. Liu, J. Yang, J. Wen, Y. Yang, X. Wei, X. Zhang, and Y.-P. Wang. Mutational analysis of dimeric linkers in peri- and cytoplasmic domains of histidine kinase DctB reveals their functional roles in signal transduction. Open Biology, 4(6): 140023-140023, 2014. ISSN 2046-2441. doi: 10.1098/rsob.140023. URL http: //rsob.royalsocietypublishing. org/cgi/doi/10.1098/rsob. 140023.

S. A. Macfarlane and M. Merrick. The nucleotide sequence of the nitrogen regulation gene ntrB and the glnA-ntrBC intergenic region of Klebsiella pneumoniae. Nucleic Acids Research, 13(21):7591-7606, 1985.

R. Malpica, B. Franco, C. Rodriguez, O. Kwon, and D. Georgellis. Identification of a quinone-sensitive redox switch in the ArcB sensor kinase. Proceedings of the National Academy of Sciences of the United States of America, 101(36): 13318-13323, 2004. ISSN 0027-8424. doi: 10.1073/pnas.0403064101. URL papers3://publication/doi/10.1073/pnas. 0403064101.

A. S. Maltsev, J. Ying, and A. Bax. Deuterium isotope shifts for backbone $1 \mathrm{H}, 15$ $\mathrm{N}$ and $13 \mathrm{C}$ nuclei in intrinsically disordered protein alpha-synuclein. Journal of biomolecular NMR, 54(2):181-191, 2012. doi: 10.1007/s10858-012-9666-x. Deuterium.

A. Marina, C. D. Waldburger, and W. A. Hendrickson. Structure of the entire cytoplasmic portion of a sensor histidine-kinase protein. EMBO Journal, 24 (24):4247-4259, 2005. ISSN 02614189. doi: 10.1038/sj.emboj.7600886. 
M. Matsushita. Histidine kinases as targets for new antimicrobial agents. Bioorganic \{\&6\} Medicinal Chemistry, 10(4):855-867, 2002. ISSN 09680896. doi: 10.1016/S0968-0896(01)00355-8. URL http://www.sciencedirect.com/ science/article/pii/S0968089601003558.

M. V. Milburn, G. G. Privé, D. L. Milligan, W. G. Scott, J. Yeh, J. Jancarik, D. E. Koshland, S.-h. Kim, M. V. Milburn, G. G. Pivi, D. L. Milligan, D. E. Koshland, and S.-h. Kim. Three-Dimensional Structures of the Ligand-Binding Domain of the Bacterial Aspartate Receptor With and Without a Ligand ThreeDimensional Structures of the Ligand-Binding Domain of the Bacterial Asp artate Receptor With and Without a Ligand. Science, 254(5036):1342-1347, 1991.

A. S. Miller and J. J. Falke. Side Chains at the Membrane - Water Interface Modulate the Signaling State of a. Biochemistry, 43(7), 2004. doi: 10.1021/ bi0360206.

H. Molinari, G. Esposito, L. Ragona, M. Pegna, N. Niccolai, R. M. Brunne, A. M. Lesk, and L. Zetta. Probing Protein Structure by Solvent Perturbation of NMR Spectra : The Surface Accessibility of Bovine Pancreatic Trypsin Inhibitor. Biophysical Journal, 73(July):382-396, 1997. doi: 10.1016/S0006-3495(97)78078-0.

K. S. Molnar, M. Bonomi, R. Pellarin, G. D. Clinthorne, G. Gonzalez, S. D. Goldberg, M. Goulian, A. Sali, and W. F. Degrado. Cys-Scanning disulfide crosslinking and bayesian modeling probe the transmembrane signaling mechanism of the histidine kinase, PhoQ. Structure, 22(9):1239-1251, 2014. ISSN 18784186. doi: 10.1016/j.str.2014.04.019. URL http://dx.doi.org/10.1016/ j.str.2014.04.019.

C. Monzel and G. Unden. Transmembrane signaling in the sensor kinase DcuS of Escherichia coli : A long-range piston-type displacement of transmembrane helix 2. Proceedings of the National Academy of Sciences of the United States of America, 112(35):11042-11047, 2015. doi: 10.1073/pnas.1507217112.

C. Monzel, P. Degreif-Dünnwald, C. Gröpper, C. Griesinger, and G. Unden. The cytoplasmic PASC domain of the sensor kinase DcuS of Escherichia coli: Role in signal transduction, dimer formation, and DctA interaction. MicrobiologyOpen, 2(6):912-927, 2013. ISSN 20458827. doi: 10.1002/mbo3.127. 
B. Morgenstern. DIALIGN: Multiple DNA and protein sequence alignment at BiBiServ. Nucleic Acids Research, 32(WEB SERVER ISS.):33-36, 2004. ISSN 03051048. doi: 10.1093/nar/gkh373.

G. Mosqueda, M. I. Ramos-González, and J. L. Ramos. Toluene metabolism by the solvent-tolerant Pseudomonas putida DOT-T1 strain, and its role in solvent impermeabilization. Gene, 232(1):69-76, 1999. ISSN 03781119. doi: 10.1016/S0378-1119(99)00113-4.

M. Müllner, O. Hammel, B. Mienert, S. Schlag, E. Bill, and G. Unden. A PAS domain with an oxygen labile [4Fe-4S]2+ cluster in the oxygen sensor kinase NreB of staphylococcus carnosus. Biochemistry, 47(52):13921-13932, 2008. ISSN 00062960. doi: 10.1021/bi8014086.

M. B. Neiditch, M. J. Federle, A. J. Pompeani, R. C. Kelly, D. L. Swem, P. D. Jeffrey, B. L. Bassler, and F. M. Hughson. Ligand-Induced Asymmetry in Histidine Sensor Kinase Complex Regulates Quorum Sensing. Cell, 126(6):1095-1108, 2006. ISSN 00928674. doi: 10.1016/j.cell.2006.07.032.

A. J. Ninfa and B. Magasanik. Covalent modification of the glnG product, NRI, by the glnL product, NRII, regulates the transcription of the glnALG operon in Escherichia coli. Proceedings of the National Academy of Sciences of the United States of America, 83(16):5909-13, 1986. ISSN 0027-8424. doi: 10.1073/ pnas.83.16.5909. URL http://www. pubmedcentral.nih.gov/articlerender . fcgi?artid=386406\{\&\}tool=pmcentrez $\{\&\}$ rendertype=abstract .

T. Ogino, M. Matsubara, N. Kato, N. Yoshihiro, and M. Takeschi. An Escherichia coli protein that exhibits phosphohistidine phosphatase activity towards the HPt domain of the ArcB sensor involved in the multistep His-Asp phosphorelay. Molecular Microbiology, 27(3):573-585, 1998.

C. Öster, S. Kosol, C. Hartlmüller, J. M. Lamley, D. Iuga, A. Oss, M. L. Org, K. Vanatalu, A. Samoson, T. Madl, and J. R. Lewandowski. Characterization of Protein-Protein Interfaces in Large Complexes by Solid-State NMR Solvent Paramagnetic Relaxation Enhancements. Journal of the American Chemical Society, 139(35):12165-12174, 2017. ISSN 15205126. doi: 10.1021/jacs.7b03875.

L. Pappalardo, I. G. Janausch, V. Vijayan, E. Zientz, J. Junker, W. Peti, M. Zweckstetter, G. Unden, and C. Griesinger. The NMR Structure of the 
Sensory Domain of the Membranous Two-component Fumarate Sensor ( Histidine Protein Kinase ) DcuS of Escherichia coli *. The journal of Biological Chemistry, 278(40):39185-39188, 2003. doi: 10.1074/jbc.C300344200.

J. S. Parkinson. Signaling Mechanisms of HAMP Domains in Chemoreceptors and Sensor Kinases. Annual Review of Microbiology, 64:101-122, 2010. doi: 10.1146/annurev.micro.112408.134215.

J. S. Parkinson, G. L. Hazelbauer, and J. J. Falke. Signaling and sensory adaptation in Escherichia coli chemoreceptors : 2015 update. Trends in Microbiology, 23(5):257-266, 2015. ISSN 0966-842X. doi: 10.1016/j.tim.2015.03.003. URL http://dx.doi.org/10.1016/j.tim.2015.03.003.

M. Perego. Kinase - phosphatase competition regulates Bacillus subtilis development. Trends in Microbiology, 6(9):366-370, 1998.

J. A. Peters, J. Huskens, and D. J. Raber. Lanthanide induced shifts and relaxation rate enhancements. Progress in Nuclear Magnetic Resonance Spectroscopy, 28 (3-4):283-350, 1996. ISSN 00796565. doi: 10.1016/0079-6565(95)01026-2.

G. Pintacuda and G. Otting. Identification of protein surfaces by NMR measurements with a paramagnetic Gd(III) chelate. Journal of the American Chemical Society, 124(3):372-373, 2002. ISSN 00027863. doi: 10.1021/ja016985h.

A. I. Podgornaia, P. Casino, A. Marina, and M. T. Laub. Structural basis of a rationally rewired protein-protein interface critical to bacterial signaling. Structure, 21(9):1636-1647, 2013. ISSN 09692126. doi: 10.1016/j.str.2013.07.005. URL http://dx.doi.org/10.1016/j.str.2013.07.005.

A. Razvi and J. M. Scholtz. Lessons in stability from thermophilic proteins. Protein Science, 15(7):1569-1578, 2006. ISSN 09618368. doi: 10.1110/ps.062130306. URL http://doi.wiley.com/10.1110/ps.062130306.

S. Reinelt, E. Hofmann, T. Gerharz, M. Bott, and D. R. Madden. The structure of the periplasmic ligand-binding domain of the sensor kinase CitA reveals the first extracellular pas domain. Journal of Biological Chemistry, 278(40):3918939196, 2003. ISSN 00219258. doi: 10.1074/jbc.M305864200.

W. Rieping, M. Habeck, B. Bardiaux, A. Bernard, T. E. Malliavin, and M. Nilges. ARIA2: automated NOE assignment and data integration in NMR structure calculation. Bioinformatics, 23(3):381-382, 2006. 
W. Rieping, B. Bardiaux, A. Bernard, T. E. Malliavin, and M. Nilges. ARIA2: Automated NOE assignment and data integration in NMR structure calculation. Bioinformatics, 23(3):381-382, 2007. ISSN 13674803. doi: 10.1093/ bioinformatics/btl589.

E. Saita, L. A. Abriata, Y. T. Tsai, F. Trajtenberg, T. Lemmin, A. Buschiazzo, M. D. Peraro, D. D. Mendoza, and D. Albanesi. A coiled coil switch mediates cold sensing by the thermosensory protein DesK. Molecular Microbiology, 98 (August):258-271, 2015. doi: 10.1111/mmi.13118.

M. Salvi, B. Schomburg, K. Giller, S. Graf, G. Unden, S. Becker, A. Lange, and C. Griesinger. Sensory domain contraction in histidine kinase CitA triggers transmembrane signaling in the membrane-bound sensor. Proceedings of the National Academy of Sciences, 114(12):3115-3120, 2017. ISSN 0027-8424. doi: 10.1073/pnas.1620286114. URL http://www .pnas .org/lookup/doi/10.1073/ pnas. 1620286114.

B. Schomburg. Transmembrane Signalling: Structural and Functional Studies on Histidine Kinase CitA. PhD thesis, 2014.

T. Schubeis, T. Le, L. B. Andreas, and G. Pintacuda. Perspectives in Magnetic Resonance $\mathrm{H}$ magic-angle spinning NMR evolves as a powerful new tool for membrane proteins. Journal of Magnetic Resonance, 287:140-152, 2017. ISSN 1090-7807. doi: 10.1016/j.jmr.2017.11.014. URL https://doi.org/10.1016/ j.jmr.2017.11.014.

M. Sevvana, V. Vijayan, M. Zweckstetter, S. Reinelt, D. R. Madden, R. HerbstIrmer, G. M. Sheldrick, M. Bott, C. Griesinger, and S. Becker. A Ligand-Induced Switch in the Periplasmic Domain of Sensor Histidine Kinase CitA. Journal of Molecular Biology, 377(2):512-523, 2008. ISSN 00222836. doi: 10.1016/j.jmb. 2008.01.024.

Y. Shen, F. Delaglio, G. Cornilescu, and A. Bax. TALOS+: a hybrid method for predicting protein backbone torsion angles from NMR chemical shifts. Journal of biomolecular NMR, 44(4):213-223, 2009.

P. A. Steinmetz, S. Wörner, and G. Unden. Differentiation of DctA and DcuS function in the DctA / DcuS sensor complex of Escherichia coli : function of DctA as an activity switch and of DcuS as the C 4 -dicarboxylate sensor. 94 (September):218-229, 2014. doi: 10.1111/mmi.12759. 
A. N. N. Stock, T. Chen, D. Welsh, and J. Stock. CheA protein, a central regulator of bacterial chemotaxis, belongs to a family of proteins that control gene expression in response to changing environmental conditions Smdi-. Proceedings of the National Academy of Sciences of the United States of America, 85 (March):1403-1407, 1988.

N. Sukomon, J. Widom, P. P. Borbat, J. H. Freed, and B. R. Crane. Stability and Conformation of a Chemoreceptor HAMP Domain Chimera Correlates with Signaling Properties. Biophysical Journal, 112(7):1383-1395, 2017. ISSN 00063495. doi: 10.1016/j.bpj.2017.02.037. URL http://dx.doi.org/10.1016/j. bpj.2017.02.037.

B. L. Taylor and I. B. Zhulin. PAS domains: internal sensors of oxygen, redox potential, and light. Microbiology and molecular biology reviews : MMBR, 63(2):479-506, 1999. ISSN 1092-2172. doi: 1092-2172/99. URL http://mmbr.asm.org/content/63/2/479. short\{\%\}5Cnhttp://www.ncbi. nlm.nih.gov/pubmed/10357859\{\%\}5Cnhttp://www . pubmedcentral.nih . gov/articlerender.fcgi?artid=PMC98974.

P. Thomason and R. Kay. Eukaryotic signal transduction via histidine-aspartate phosphorelay. Journal of cell science, 113 ( Pt 1:3141-50, 2000. ISSN 0021-9533. URL http://www.ncbi.nlm.nih.gov/pubmed/10954413.

F. Trajtenberg, M. Graña, N. Ruétalo, H. Botti, and A. Buschiazzo. Structural and enzymatic insights into the ATP binding and autophosphorylation mechanism of a sensor histidine kinase. Journal of Biological Chemistry, 285(32):24892-24903, 2010. ISSN 1083351X. doi: 10.1074/jbc.M110.147843.

L. E. Ulrich and I. B. Zhulin. The MiST2 database : a comprehensive genomics resource on microbial signal transduction. Nucleic Acids Research, 38(September): 401-407, 2018. doi: 10.1093/nar/gkp940.

P. Vallurupalli, A. Sekhar, T. Yuwen, and L. E. Kay. Probing conformational dynamics in biomolecules via chemical exchange saturation transfer: a primer. Journal of Biomolecular NMR, 67(4):243-271, 2017. ISSN 15735001. doi: 10. 1007/s10858-017-0099-4.

W. F. Vranken, W. Boucher, T. J. Stevens, R. H. Fogh, A. Pajon, M. Llinas, E. L. Ulrich, J. L. Markley, J. Ionides, and E. D. Laue. The CCPN data model for NMR spectroscopy: Development of a software pipeline. Proteins: 
Structure, Function and Genetics, 59(4):687-696, 2005. ISSN 08873585. doi: $10.1002 /$ prot.20449.

J. Vreede, M. A. Van der Horst, K. J. Hellingwerf, W. Crielaard, and D. M. Van Aalten. PAS domains. Common structure and common flexibility. Journal of Biological Chemistry, 278(20):18434-18439, 2003. ISSN 00219258. doi: 10.1074/ jbc.M301701200.

B. Wang, A. Zhao, R. P. Novick, and T. W. Muir. Article Activation and Inhibition of the Receptor Histidine Kinase AgrC Occurs through Opposite Helical Transduction Motions. Molecular Cell, 53(6):929-940, 2014. ISSN 1097-2765. doi: 10.1016/j.molcel.2014.02.029. URL http://dx.doi.org/10.1016/j.molcel. 2014.02 .029 .

C. Wang, J. Sang, J. Wang, M. Su, J. S. Downey, Q. Wu, S. Wang, Y. Cai, X. Xu, J. Wu, D. B. Senadheera, D. G. Cvitkovitch, L. Chen, S. D. Goodman, and A. Han. Mechanistic Insights Revealed by the Crystal Structure of a Histidine Kinase with Signal Transducer and Sensor Domains. PLoS Biology, 11(2), 2013. ISSN 15449173. doi: 10.1371/journal.pbio.1001493.

J. Wang, J. Sasaki, A.-l. Tsai, and J. L. Spudich. HAMP Domain Signal Relay Mechanism in a Sensory Rhodopsin-Transducer Complex *. The journal of Biological Chemistry, 287(25):21316-21325, 2012. doi: 10.1074/jbc.M112.344622.

Y. Wang and O. Jardetzky. Probability-based protein secondary structure identification using combined NMR chemical-shift data. Protein Science, 11:852-861, 2002. doi: 10.1110/ps.3180102.Structure.

S. Weisenburger, D. Boening, B. Schomburg, K. Giller, S. Becker, C. Griesinger, and V. Sandoghdar. Cryogenic optical localization provides 3D protein structure data with Angstrom resolution. Nature Methods, 14(2):141-144, 2017. ISSN 15487105. doi: 10.1038/nmeth.4141.

F. H. Westheimer. Nature Chose Phosphates The Role of Phosphates The Importance of Being Ionized. Science (New York, N.Y.), 235(1):1173-1178, 1987. ISSN 0036-8075. doi: 10.1126/science.2434996.

D. White, R. J. Sharp, and F. G. Priest. A polyphasic taxonomic study of thermophilic bacilli from a wide geographical area. Antonie van Leeuwenhoek, 64(3):357-386, sep 1993. ISSN 1572-9699. doi: 10.1007/BF00873093. URL https://doi .org/10.1007/BF00873093. 
D. S. Wishart, B. D. Sykes, and F. M. Richard. Relationship between Nuclear Magnetic Resonance Chemical Shift and Protein Secondary Structure. Journal of Molecular Biology, 222:311-333, 1991.

R. Wu, M. Gu, R. Wilton, G. Babnigg, Y. Kim, P. R. Pokkuluri, H. Szurmant, A. Joachimiak, and M. Schiffer. Insight into the sporulation phosphorelay: Crystal structure of the sensor domain of Bacillus subtilis histidine kinase, KinD. Protein Science, 22(5):564-576, 2013. ISSN 09618368. doi: 10.1002/pro.2237.

D. H. Zhou and C. M. Rienstra. High-performance solvent suppression for proton detected solid-state NMR. Journal of Magnetic Resonance, 192(1):167-172, 2008. ISSN 10907807. doi: 10.1016/j.jmr.2008.01.012.

Q. Zhou, P. Ames, and J. S. Parkinson. Mutational analyses of HAMP helices suggest a dynamic bundle model of input - output signalling in chemoreceptors. Molecular Microbiology, 73(August):801-814, 2009. doi: 10.1111/j.1365-2958. 2009.06819.x.

Y. F. Zhou, B. Nan, J. Nan, Q. Ma, S. Panjikar, Y. H. Liang, Y. Wang, and X. D. Su. C4-Dicarboxylates Sensing Mechanism Revealed by the Crystal Structures of DctB Sensor Domain. Journal of Molecular Biology, 383(1):49-61, 2008. ISSN 00222836. doi: 10.1016/j.jmb.2008.08.010.

E. Zientz, J. Bongaerts, G. Unden, and J. Gutenberg. Fumarate Regulation of Gene Expression in Escherichia coli by the DcuSR ( dcuSR Genes ) TwoComponent Regulatory System. 180(20):5421-5425, 1998. 